\title{
Extensional Flow Blending of Immiscible Polymers with Nanoparticle Stabilization
}

\author{
Matthew S. Thompson
}

Follow this and additional works at: https://researchrepository.wvu.edu/etd

\section{Recommended Citation}

Thompson, Matthew S., "Extensional Flow Blending of Immiscible Polymers with Nanoparticle Stabilization" (2016). Graduate Theses, Dissertations, and Problem Reports. 6801.

https://researchrepository.wvu.edu/etd/6801

This Dissertation is protected by copyright and/or related rights. It has been brought to you by the The Research Repository @ WVU with permission from the rights-holder(s). You are free to use this Dissertation in any way that is permitted by the copyright and related rights legislation that applies to your use. For other uses you must obtain permission from the rights-holder(s) directly, unless additional rights are indicated by a Creative Commons license in the record and/ or on the work itself. This Dissertation has been accepted for inclusion in WVU Graduate Theses, Dissertations, and Problem Reports collection by an authorized administrator of The Research Repository @ WVU.

For more information, please contact researchrepository@mail.wvu.edu. 


\title{
Extensional Flow Blending of Immiscible Polymers with Nanoparticle Stabilization
}

\author{
Matthew S. Thompson \\ Dissertation submitted to the \\ Benjamin M. Statler College of Engineering and Mineral Resources \\ at West Virginia University \\ in partial fulfillment of the requirements \\ for the degree of \\ Doctor of Philosophy \\ in \\ Chemical Engineering \\ Rakesh K. Gupta, Ph.D., Chair \\ Sushant Agarwal, Ph.D. \\ Pradeep P. Fulay, Ph.D. \\ Robin S. Hissam, Ph.D. \\ Pierre Moulinié, Ph.D. \\ Xueyan Song, Ph.D. \\ Charter D. Stinespring, Ph.D. \\ Department of Chemical and Biomedical Engineering \\ Morgantown, West Virginia \\ 2016
}

Keywords: Polymer blends, morphology development, processing-structure relationships, converging flow, extensional flow cells, drop dispersion, nanoparticle stabilization, compatibilization, fumed nanosilica

Copyright 2016 Matthew S. Thompson 


\section{ABSTRACT \\ Extensional Flow Blending of Immiscible Polymers with Nanoparticle Stabilization Matthew S. Thompson}

Polymer blending facilitates the combination of the attractive attributes of two or more polymers while compensating for the unfavorable ones. Most polymers are thermodynamically incompatible with one another, and their blending yields a two-phase microstructure. This morphology generally determines the mechanical and rheological properties of the blend system which then determine its applications. Morphology development typically involves deformation of the dispersed phase followed by drop breakup. However, drop coalescence competes with this process, and ultimately a balance must be reached between these two competing processes. Extensional flow fields are known to promote drop breakup and are especially important for blends with high viscosity ratios, that is for blends where the viscosity of the dispersed phase is at least about 3.8 times greater than that of the matrix phase. Coalescence may be attenuated by compatibilizers that modify the interface between the polymer phases. Nanoparticles with tuned surface chemistry may also be used as compatibilizers. A combination of extensional flow and nanoparticle stabilization should, therefore, result in a fine, stable morphology.

To begin the investigation toward the effects of extensional flow blending with and without the incorporation of nanoparticles, preliminary results were obtained using two different polymer blend systems: polycarbonate (PC)/styrene acrylonitrile (SAN) and polystyrene (PS)/linear low-density polyethylene (LLDPE). However, the majority of the presented results involve blends of high-density polyethylene (HDPE) dispersed in PS. With this blend system, with the material grades selected, the viscosity ratio exceeded 3.8 over the entire domain of deformation rates anticipated in the processing used. Coarse blends of various compositions were formulated using shear flow in an internal mixer or in a twin-screw extruder. These blends were subjected to extensional flow in converging dies of different geometries and where more than one stretching episode was possible; the temperature, total strain, and flow rate were varied, among other factors, in a systematic manner. Experiments were repeated in the presence of various grades of fumed nanosilica of different sizes and surface treatments, which imparted different surface tension and relative surface polarity (hydrophilic versus hydrophobic) for the nanoparticles. The mixing sequence was varied including premixing the nanosilica into the thermodynamically non-preferred polymer phase.

Scanning electron microscopy (SEM) was used to determine the size and size distribution of the dispersed polymer phase. The material was typically sectioned in the flow direction, but sectioning in the direction perpendicular to flow and etching, or selectively dissolving, one phase or the other was also investigated. The primary effect of extensional flow blending was to reduce the volume-average diameter of the dispersed polymer phase, especially with increasing strains and flow rates, or strain rates, which is directly dependent on both. Finding suitable conditions for the nanoparticles to selectively localize at the HDPE/PS interface was challenging, but relatively small amounts of nanoparticles dispersed in the PS matrix decreased the volume-average diameter of HDPE drops. When the nanosilica was preloaded into the HDPE dispersed phase, very coarse initial blends were produced which then exhibited dramatic decreases in phase size with extensional flow. These and other results are properly organized and presented. 
To my wife, Kayla, without whose patience, love, and support this dissertation would not have been possible 


\section{ACKNOWLEDGEMENTS}

The credit and glory associated with this research and this dissertation go to God, from whom my engineering intellect, wisdom, and work ethic derive and for whose purpose this was achieved. My sincere thanks go to my family, friends, professors, colleagues, and peers who encouraged me and gave me valuable advice toward the completion of this research and the tome that you are reading. Thanks to my wife Kayla, who is my motivator, friend, scheduling assistant, confidant, sounding board, domestic commander, partner, and person. Kayla you are an amazing and incredibly smart person, and I am blessed to have you in my life, especially in such capacity as my wife. Thanks to my son Brady, who is also a significant motivator for me to forge the path for a better life for my family. Thanks to my mom, dad, siblings, grandparents, in-laws, and the rest of my family for your undying devotion and faith in me. Thanks to my friends: my fellow classmates, my professors, my industry colleagues, and my peers; you are an inspiration and a supporting force that propels me to be a better scientist and engineer. Thanks to West Virginia University, Covestro (formerly Bayer MaterialScience), and The Boeing Company for the opportunities and incredible education that I have had the fortune of enjoying.

Thanks Dr. Rakesh Gupta for your mentorship and your confidence in me. You have taught me more about chemical and polymer engineering than I could have ever imagined, and I believe that you have helped pave the way for a great, fulfilling, and meaningful career for me. I truly appreciate your contributions to my education and early career, including your academic, industrial, and life advice; your teaching; your faith in me, which inspired me to be a selfinspired and self-confident scientist; and your caring friendship, which always made me feel comfortable and able. Thanks to Dr. Sushant Agarwal for your advice and training in the lab. You have had a significant effect on my education and early career, and I truly appreciate your inputs to my projects. Thanks to my labmates: Dr. Adam Finniss, Dr. Elliot Roth, Man Chio "Steve" Tang, Peter Welcker, Dr. Daoyun Song, Kisheon Alexander, Julie Peng, and Fei Gao. Adam, I truly appreciate your friendship, in-depth polymer science discussions, and training and education in the lab. Elliot, thanks for your inspirations and motivation toward my research. 
Thanks Steve for your friendship. Thanks Peter for your enthusiasm and hard work in helping me with my research, especially toward the investigation of the effects of the incorporation of nanoparticles in polymer blends.

Thanks to my friends and colleagues in academia and industry. Thanks Jeremy Hardinger, Andrew Graves, Alixandra Wagner, Kelydra Welcker, Trey Menincosa, Bill Kistler, and Jacob Weidman for your friendship and intellectual conversations. Thanks to my committee members, Dr. Charter Stinespring, Dr. Robin Hissam, Dr. Pierre Moulinié, Dr. Xueyan Song, and Dr. Pradeep Fulay for your contributions to the project and my early career. Thanks to Dr. Peter Uthe and Dr. Marina Rogunova for your mentorship and leadership in my internship experiences at Bayer MaterialScience (now Covestro). Thanks to John Waldrop, Loren Strahm, Chris Robinson, Keith Rupel, Mike Hayes, Dan Braley, Eric Deck, and Garrett Peters for your aid in my acclamation to industrial research and its corresponding enhancement to my career at The Boeing Company.

Finally, thanks to Bayer MaterialScience (now Covestro), the George \& Carolyn Berry Endowment Chair, and the Benjamin M. Statler College of Engineering and Mineral Sciences for funding this project through the Bayer Fellowship at West Virginia University (WVU), Berry Endowment Chair, and Statler PhD Fellowship. 


\section{TABLE OF CONTENTS}

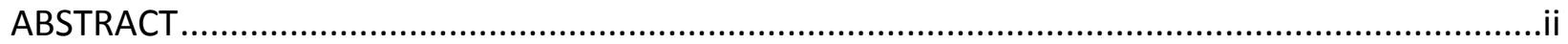

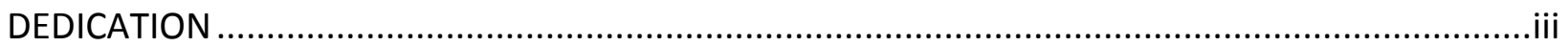

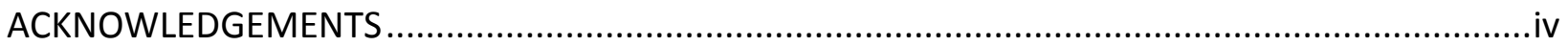

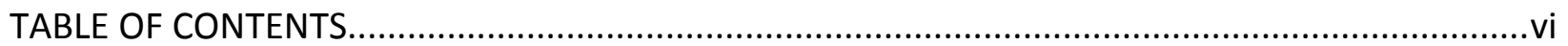

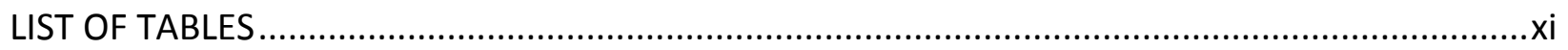

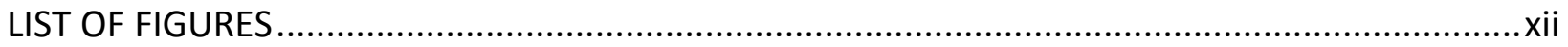

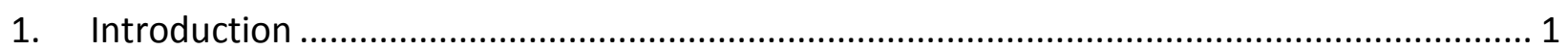

1.1 Brief Background and Problem Statement ....................................................... 1

$1.2 \quad$ Brief Description of Experimental Methods ........................................................ 4

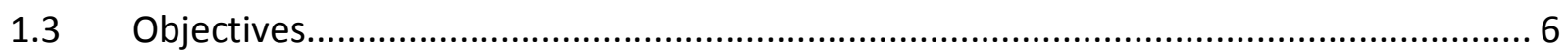

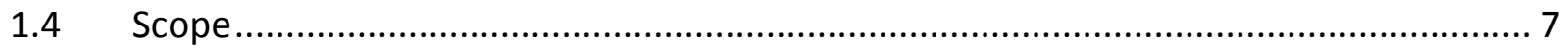

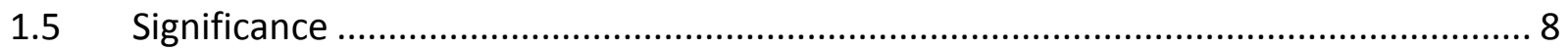

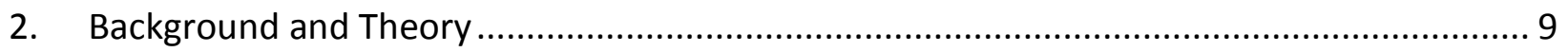

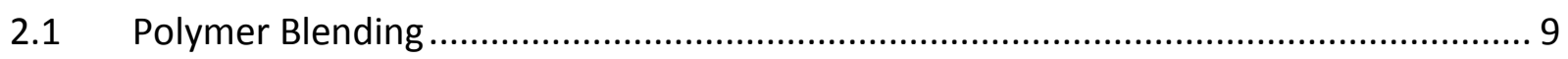

2.1.1 Thermodynamics of Polymer Blends ........................................................... 10

2.1.2 Kinetics during Processing of Blends ............................................................. 12

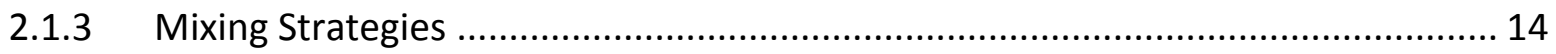

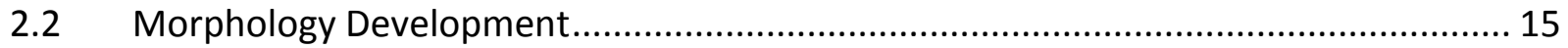

2.2.1 Description of Morphologies ......................................................................... 15

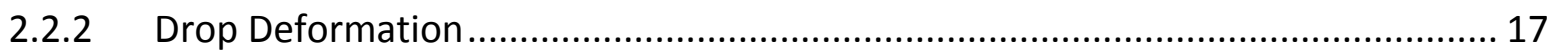

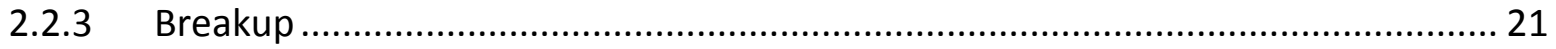

2.2.3.1 Breakup of Newtonian Thread in Quiescent Newtonian Matrix.................... 21

2.2.3.2 Breakup of Viscoelastic Thread in a Quiescent Matrix............................... 25 
2.2.3.3 Breakup under Flow .......................................................................... 26

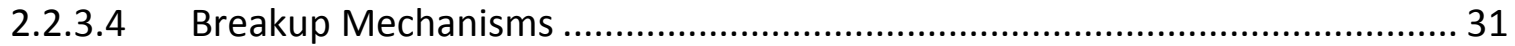

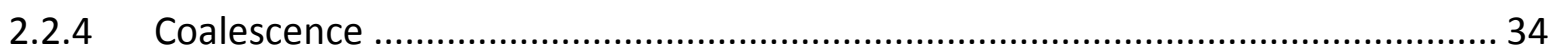

2.2.5 Overall Morphology Development for Viscoelastic Blends ................................... 42

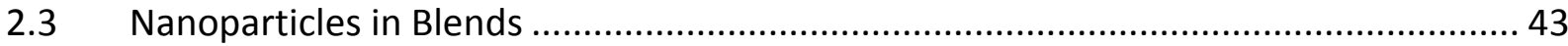

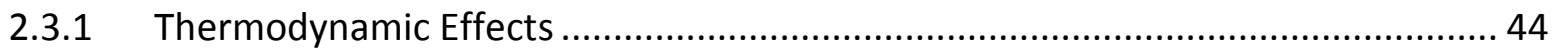

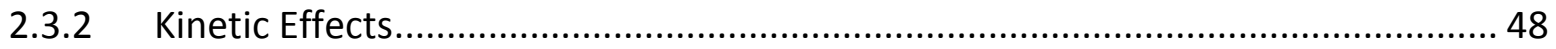

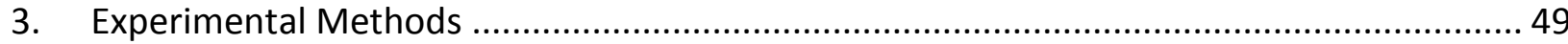

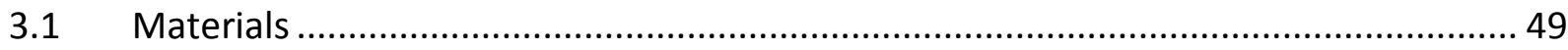

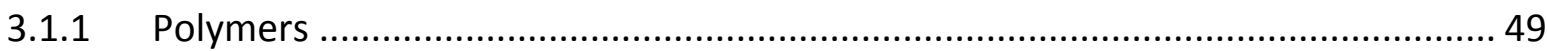

3.1.1.1 Preliminary Blends PC/SAN and PS/LLDPE .................................................. 49

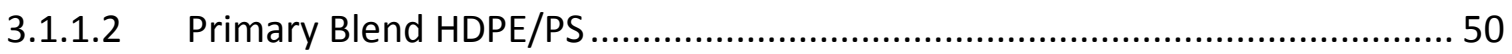

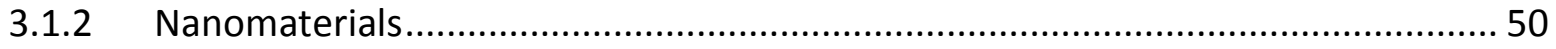

3.2 Materials Characterization Procedures .................................................................. 53

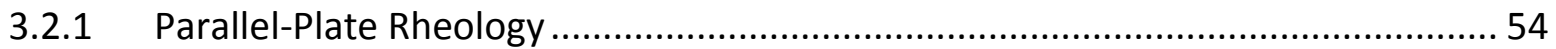

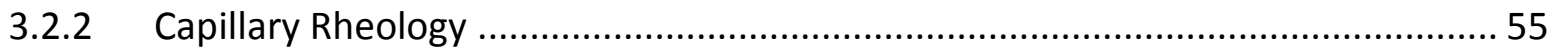

3.2.3 Differential Scanning Calorimetry.................................................................. 57

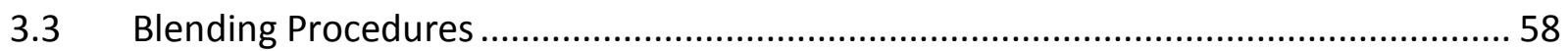

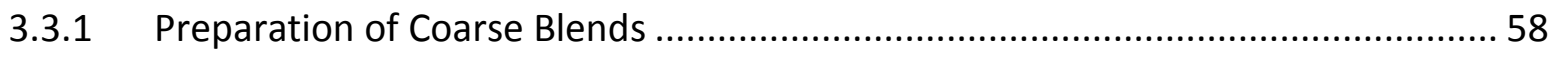

3.3.1.1 Unfilled Coarse Blends.............................................................................. 59

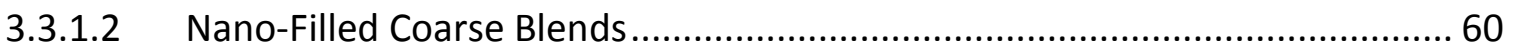

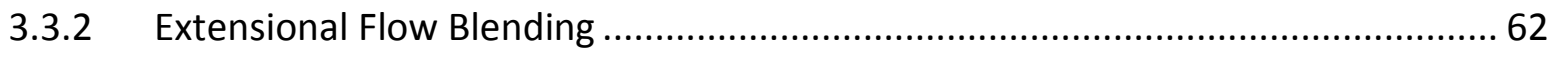

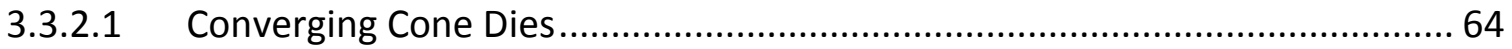

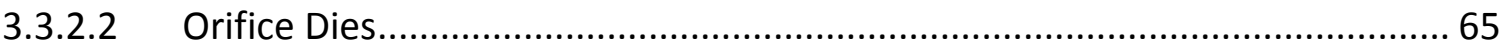




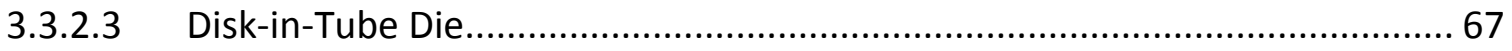

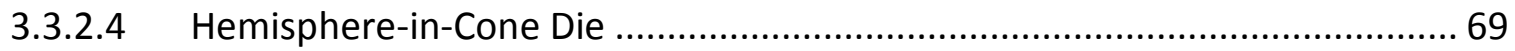

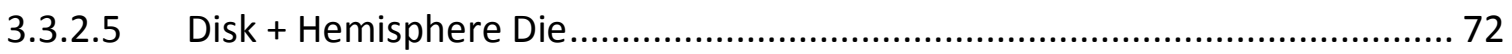

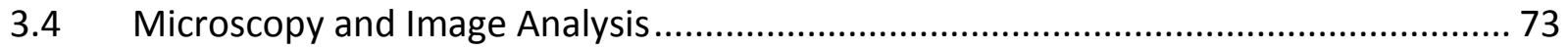

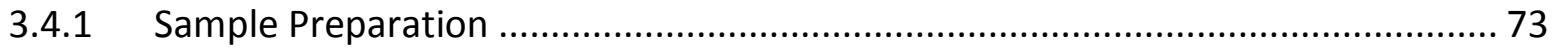

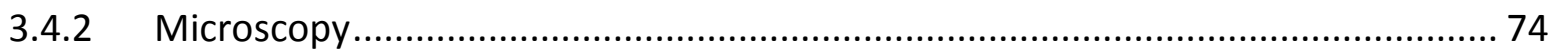

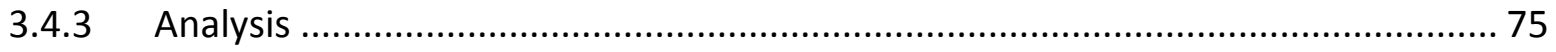

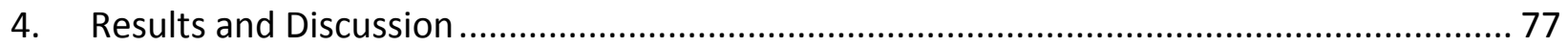

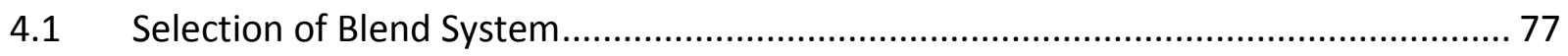

4.1.1 Polycarbonate (PC)/Styrene Acrylonitrile (SAN) .......................................... 77

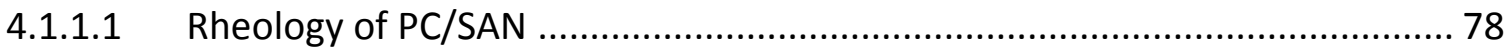

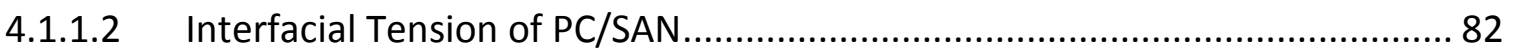

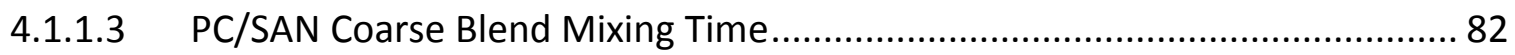

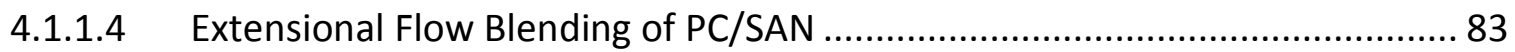

4.1.1.4.1 PC/SAN through Orifice Dies.......................................................... 83

4.1.1.4.2 PC/SAN through Converging Cone Dies ............................................ 93

4.1.2 Polystyrene (PS)/Linear Low-Density Polyethylene (LLDPE) ............................... 94

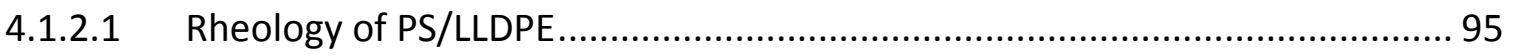

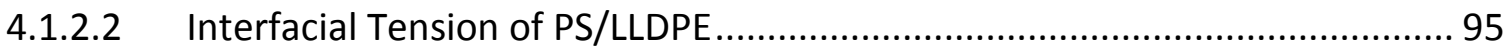

4.1.2.3 PS/LLDPE Coarse Blend Mixing Time ..................................................... 98

4.1.2.4 Extensional Flow Blending of PS/LLDPE through Hemisphere-in-Cone Dies 100

4.1.3 High-Density Polyethylene (HDPE)/Polystyrene (PS) ..................................... 104

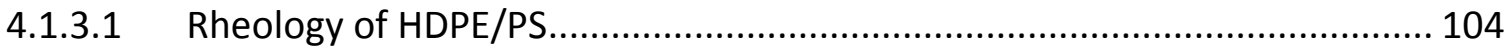

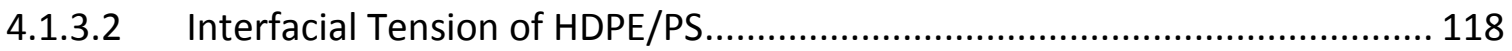


4.1.3.3 Differential Scanning Calorimetry (DSC) of HDPE/PS 120

4.1.4 Conclusions for Blend Selection.............................................................. 123

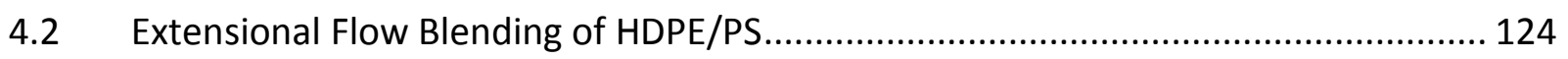

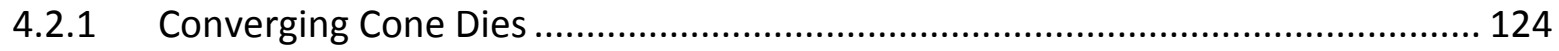

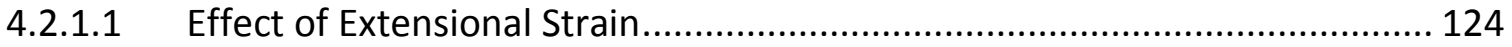

4.2.1.2 Morphological Comparisons to the Literature ..................................... 130

4.2.1.3 Effects of Flow Rate \& Extensional Strain Rate ..................................... 132

4.2.1.4 Morphological Comparisons to Theory ................................................. 140

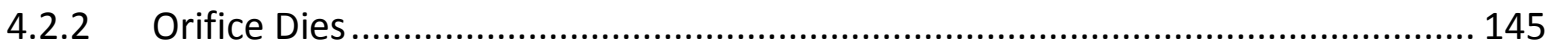

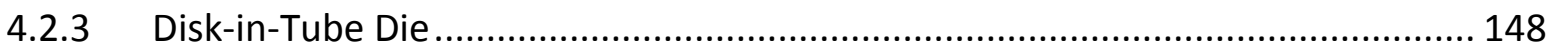

4.2.4 Hemisphere-in-Cone Dies .......................................................................... 152

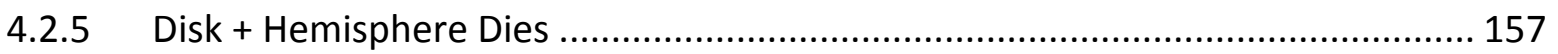

4.2.6 Selective Dissolution of the PS Matrix .......................................................... 160

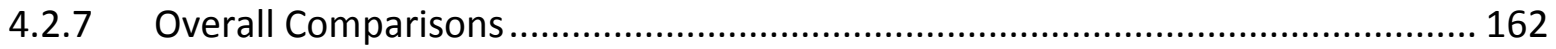

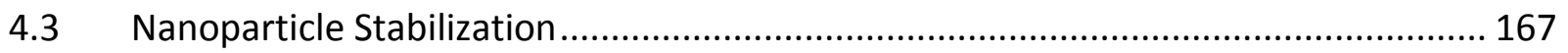

4.3.1 Properties of Fumed Nanosilica............................................................. 167

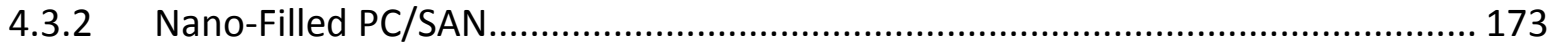

4.3.2.1 Thermodynamics of Nano-Filled PC/SAN ............................................ 173

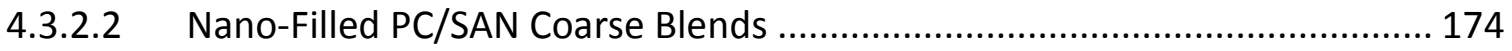

4.3.2.3 Extensional Flow with Nanoparticle Stabilization in PC/SAN ...................... 176

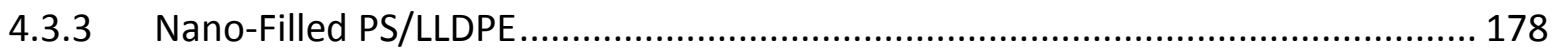

4.3.3.1 Thermodynamics of Nano-Filled PS/LLDPE ........................................... 178

4.3.3.2 Nano-Filled PS/LLDPE Coarse Blends.................................................... 179

4.3.3.3 Extensional Flow with Nanoparticle Stabilization in PS/LLDPE ................... 180 


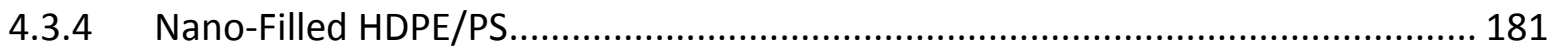

4.3.4.1 Thermodynamics of Nano-Filled HDPE/PS ........................................... 181

4.3.4.2 Nano-Filled HDPE/PS Coarse Blends .................................................... 184

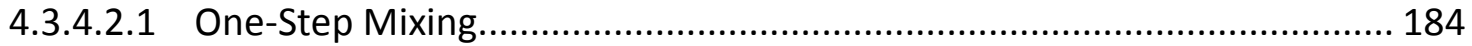

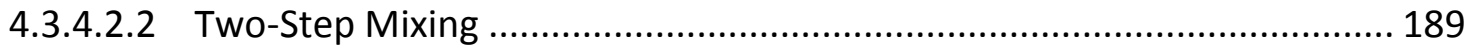

4.3.4.3 Extensional Flow with Nanoparticle Stabilization ................................... 195

4.3.5 Conclusions for Nanoparticle Stabilization .................................................... 205

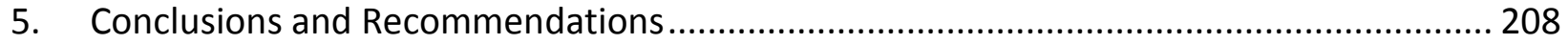

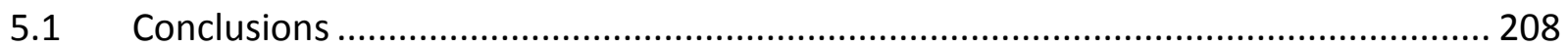

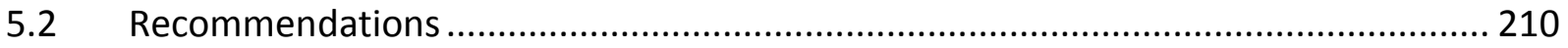

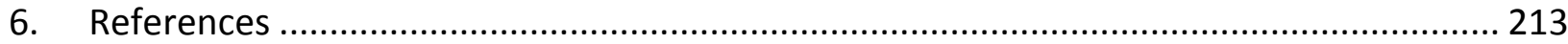




\section{LIST OF TABLES}

Table 2-1: Film drainage models for different interface mobility assumptions ........................ 36

Table 2-2: Conditions to deviate from partially mobile interface model ................................ 38

Table 2-3: Drainage probability for various interfacial mobilities ............................................ 39

Table 2-4: Maximum drop size for coalescence …............................................................ 42

Table 3-1: Fumed silica grades used in this work .................................................................... 52

Table 3-2: Dispersed-phase concentration conversions for selected blend compositions......... 60

Table 3-3: Concentration conversions for nano-filled 5\% HDPE/PS blends ............................. 62

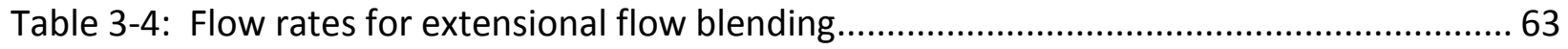

Table 3-5: Extensional strain through hemisphere-in-cone die .......................................... 71

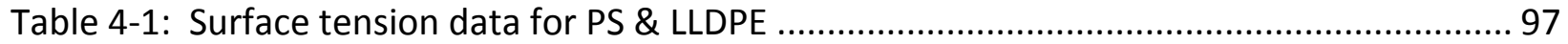

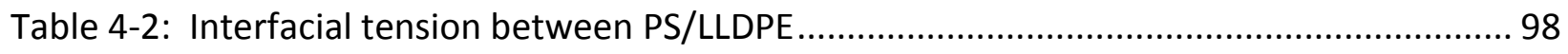

Table 4-3: Melt flow indices of PS grades ........................................................................ 106

Table 4-4: Melt flow indices of PE grades ..................................................................... 107

Table 4-5: Measured zero-shear viscosity for PE and PS grades ......................................... 108

Table 4-6: Rheological fit parameters for HDPE \& PS ....................................................... 113

Table 4-7: Crossover points and relaxation times from rheology measurements .................. 117

Table 4-8: Surface tension data for HDPE \& PS ............................................................. 119

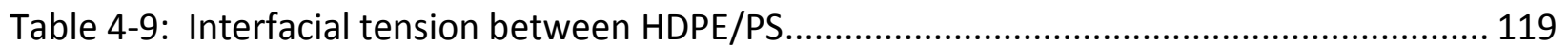

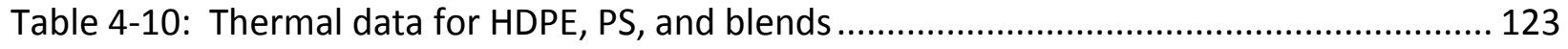

Table 4-11: Dimensionless numbers for extensional strain through converging cone dies ..... 128

Table 4-12: Morphology using converging cone dies at $17 \mathrm{~cm}^{3} / \mathrm{min}$................................. 131

Table 4-13: Dimensionless numbers for various flow rates through converging cone die ....... 137

Table 4-14: Morphology comparisons to theory for $0.559 \mathrm{~mm}$ converging cone die.............. 143

Table 4-15: Morphology comparisons to theory for $0.330 \mathrm{~mm}$ converging cone die.............. 144

Table 4-16: Flow field characteristics for disk-in-tube die................................................... 150

Table 4-17: Properties of nanosilica grades................................................................... 170

Table 4-18: Nanosilica localization in one step-mixed coarse blends ................................... 187

Table 4-19: Nanosilica localization in two step-mixed coarse blends .................................. 190 


\section{LIST OF FIGURES}

Figure 1-1: High-level goal of research - Processing-Structure-Properties Relationships ........... 3

Figure 1-2: Patented extensional flow mixing devices .................................................... 8

Figure 2-1: Polymer blending potential effects on properties ............................................ 10

Figure 2-2: Shear flow and extensional flow field schematics............................................ 13

Figure 2-3: Two-phase morphologies .............................................................................. 16

Figure 2-4: Critical capillary number as a function of flow type \& viscosity ratio ...................... 18

Figure 2-5: Drop deformation in different types of flow ....................................................... 20

Figure 2-6: Sinusoidal disturbance on thread surface ........................................................ 22

Figure 2-7: Dominant growth rate \& wave number vs. viscosity ratio................................... 23

Figure 2-8: Breakup of a Newtonian thread in a quiescent Newtonian matrix........................ 24

Figure 2-9: Breakup of a viscoelastic thread in a quiescent Newtonian matrix ....................... 25

Figure 2-10: Dimensionless critical thread radius vs. dimensionless stretch rate $\& p$............... 28

Figure 2-11: Dimensionless droplet radius vs. dimensionless stretch rate \& viscosity ratio ...... 29

Figure 2-12: Fatal disturbance growth time vs. dimensionless stretch rate $\&$ viscosity ratio .... 30

Figure 2-13: Schematic representations of stepwise binary breakup \& capillary breakup........ 32

Figure 2-14: Minimum drop size in plane hyperbolic extensional flow .................................. 33

Figure 2-15: Interface mobility during film drainage ....................................................... 35

Figure 2-16: Probabilities associated with coalescence vs. radius \& flow parameter ............... 40

Figure 2-17: Coalescence probability vs. capillary number for various drop radii .................... 41

Figure 2-18: Schematic of nanoparticle at liquid-liquid interface ............................................ 45

Figure 2-19: Surface Gibbs free energy for a nanoparticle at interface .................................. 46

Figure 3-1: PS and HDPE pellets and nanosilica powders ................................................... 52

Figure 3-2: Rheometrics RMS-800 rotational rheometer .................................................. 55

Figure 3-3: Bohlin Rosand RH2000 capillary rheometer.................................................... 56

Figure 3-4: Internal mixer with roller rotors ................................................................. 59

Figure 3-5: High-level schematic of blend compositions and nanosilica grade characteristics .. 61 
Figure 3-6: Schematic representations of the converging cone dies 65

Figure 3-7: Schematic representations of the orifice dies 67

Figure 3-8: Schematic representations of the disk-in-tube die..... 68

Figure 3-9: Schematic representations of the hemisphere-in-cone die. 69

Figure 3-10: Cross-sectional areas for flow through hemisphere-in-cone die 71

Figure 3-11: Schematic representation of the disk + hemisphere die 72

Figure 4-1: PC and SAN viscosities and blend viscosity ratio. 79

Figure 4-2: 5\% PC/SAN coarse blend. 81

Figure 4-3: Etching PC dispersed phase in 5\% PC/SAN. 81

Figure 4-4: Effect of mixing time on morphology of 1\% PC/SAN 83

Figure 4-5: SEM of 5\% PC/SAN with various extensional strains through orifice dies..... 85

Figure 4-6: Effect of extensional strain for $5 \%$ PC/SAN through orifice dies...... 86

Figure 4-7: PC/SAN phase size with extensional flow through orifice dies.... 88

Figure 4-8: PC/SAN phase size distribution with extensional flow through orifice dies 89

Figure 4-9: Schematics of coalescence during and after extensional flow. 90

Figure 4-10: Effect of PC/SAN composition on morphology . 91

Figure 4-11: SEM of 15\% PC/SAN after extensional flow polished in longitudinal direction ...... 92

Figure 4-12: Measure of best fit ellipses for polished 15\% PC/SAN after extensional flow....... 93

Figure 4-13: Morphology of 5\% PC/SAN after extensional flow through converging cone die .. 94

Figure 4-14: PS and LLDPE viscosities and blend viscosity ratio. .95

Figure 4-15: Effect of mixing time on morphology of 15\% PS/LLDPE.................................. 100

Figure 4-16: Morphological effects of 5\% PS/LLDPE using hemisphere-in-cone die................ 102

Figure 4-17: Morphological effects of 15\% PS/LLDPE using hemisphere-in-cone die.............. 103

Figure 4-18: Viscosity curves for PS and LLDPE with typical process shear rate ranges ........... 105

Figure 4-19: Shear viscosity curves of PS grades .............................................................. 106

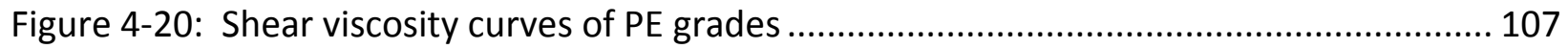

Figure 4-21: Shear viscosity curves for PS 685D and MDPE 2316 ........................................ 109

Figure 4-22: Shear viscosity curves for HDPE HBW355A and PS XU36305 …...................... 110

Figure 4-23: Shear viscosity of HDPE and PS compared to that of blends at $220^{\circ} \mathrm{C} \ldots \ldots \ldots \ldots \ldots 111$ 
Figure 4-24: Shear viscosity ratio of HDPE/PS 112

Figure 4-25: Rheological fits and viscosity ratio vs. shear stress .... 113

Figure 4-26: Dynamic rheology of HDPE HB-W355-A at $220^{\circ} \mathrm{C}$ 115

Figure 4-27: Dynamic rheology of PS XU36305 at $220^{\circ} \mathrm{C}$ 116

Figure 4-28: Dynamic rheology of $5 \% \mathrm{HDPE} / \mathrm{PS}$ at $220^{\circ} \mathrm{C}$ 117

Figure 4-29: DSC curves for HDPE, PS, and blends 122

Figure 4-30: Effect of extensional strain in converging cone dies on morphology 126

Figure 4-31: Effect of total strain on Ca for converging cone dies 127

Figure 4-32: Effect of flow rate through converging cone dies on morphology 133

Figure 4-33: Entry profiles studied by Meller et al. 2002 135

Figure 4-34: Effect of flow rate on Ca for converging cone dies 136

Figure 4-35: Morphology after extensional flow through converging cone dies 139

Figure 4-36: Pressure drop for extensional flow through converging cone dies 140

Figure 4-37: 5\% HDPE/PS morphology after flow through orifice dies 146

Figure 4-38: Log-normal morphology after flow through orifice dies 147

Figure 4-39: Pressure drop and melt fracture for flow through orifice dies 148

Figure 4-40: Effects of flow through disk-in-tube die on morphology 151

Figure 4-41: Pressure drop for flow through disk-in-tube die..... 152

Figure 4-42: Extensional strains through hemisphere-in-cone die 153

Figure 4-43: Average extensional strain rates through hemisphere-in-cone die...... 154

Figure 4-44: Effects of flow through hemisphere-in-cone die on morphology 155

Figure 4-45: Pressure drop through hemisphere-in-cone die 156

Figure 4-46: Effects of flow through disk+ hemisphere die on morphology 158

Figure 4-47: Pressure drop through disk + hemisphere die 160

Figure 4-48: HDPE drops and thin threads after orifice, $0.559 \mathrm{~mm}, 17 \mathrm{~cm}^{3} / \mathrm{min}$ 161

Figure 4-49: HDPE drops and threads after hemisphere-in-cone, 0.004" spacer, $17 \mathrm{~cm} 3 / \mathrm{min} 161$ Figure 4-50: Tip streaming on an HDPE thread after orifice, $0.559 \mathrm{~mm}, 17 \mathrm{~cm}^{3} / \mathrm{min}$ 162

Figure 4-51: Comparison of morphology after blending through all of the EFCs..... 165

Figure 4-52: Comparison of pressure drop after blending through all of the EFCs..... 166 
Figure 4-53: As-received Aerosil ${ }^{\circledR}$ R104 in agglomerated form............................................. 168

Figure 4-54: Predicted loading of nanosilica required to cover the HDPE/PS interface ........... 172

Figure 4-55: Predicted wettability of nanoparticles in PC/SAN ............................................... 174

Figure 4-56: TEM of 2\% nanosilica in 15\% PC/SAN coarse blends ....................................... 175

Figure 4-57: Morphology of nanosilica-filled 15\% PC/SAN coarse blends ............................... 176

Figure 4-58: Morphology of nanosilica-filled 15\% PC/SAN with extensional flow ..................... 177

Figure 4-59: Wettability parameter of nanoparticles with various surfaces in PS/LLDPE ........ 178

Figure 4-60: Representative TEM images of nano-filled PS/LLDPE coarse blends .................... 179

Figure 4-61: Extensional flow blending with nanoparticle stabilization of PS/LLDPE ............... 180

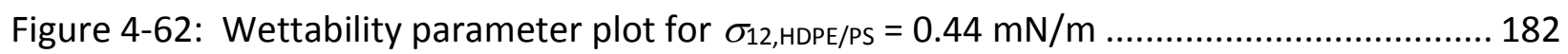

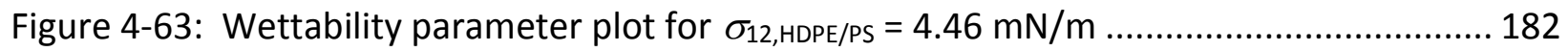

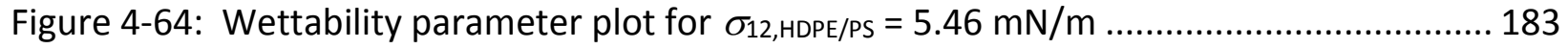

Figure 4-65: Representative EDS map of one-step mixed nano-filled blend ............................ 188

Figure 4-66: Representative TEM images of one step-mixed coarse blends ............................ 188

Figure 4-67: Representative EDS map of two-step mixed nano-filled blend ............................ 191

Figure 4-68: Comparison of EDS maps of two-step mixed nano-filled blends .......................... 192

Figure 4-69: Effect of mixing procedure on coarse blend morphology................................... 193

Figure 4-70: Complex shear viscosity vs. frequency for nano-filled blends ............................. 194

Figure 4-71: Storage and loss modulus vs. frequency for nano-filled blends .......................... 194

Figure 4-72: Representative TEM image of one step-mixed blend after extensional flow....... 196

Figure 4-73: Morphology of unfilled \& one step-mixed blends after extensional flow ............. 198

Figure 4-74: Morphology of unfilled \& nano-filled blends after extensional flow ..................... 200

Figure 4-75: Morphology of nano-filled blends through all dies ................................................. 201

Figure 4-76: Log-normal morphology of nano-filled blends through all dies............................ 202

Figure 4-77: Migration of nanoparticles to interface after extensional flow blending .............. 204 


\section{Introduction}

\subsection{Brief Background and Problem Statement}

Mixing and blending operations are prolific in the polymers industry today. To create new polymeric product offerings, materials providers rely on compounding techniques rather than polymerization technologies. This is because the massive research and development and capital expenditures required to produce new polymers or copolymers often restrict this approach's economic feasibility. Alternatively, compounding existing materials to make new products with equivalent or superior properties is an attractive route that usually requires a much smaller investment cost. Compounding may involve of the blending of various polymers together and/or the mixing of other fillers, reinforcements, and additives into the formulated product. One example where this may result in a superior combination of properties is impactmodified blends, in which a rubber impact modifier is blended into a high-strength and highmodulus, but inherently brittle plastic in such a way to impart toughness and impact resistance without detrimentally affecting the inherently attractive properties of the matrix plastic. The challenges that must be overcome to successfully accomplish this include the incompatibility of most polymers with most other polymers and the difficulties associated with effectively dispersing and distributing secondary phases into a polymer matrix. The purpose of this dissertation is to detail a few physical and thermodynamic approaches that may be employed to successfully create high-quality blended polymer systems.

Blending of polymers is often challenging when the polymers are incompatible physically and thermodynamically. Physical incompatibility is often encountered when the viscosity of the polymer one is attempting to disperse into the matrix is much greater than that of the matrix-phase polymer. It is also exacerbated when the dispersed phase is highly elastic, which must be considered in transient operations, that is processing operations which do not reach a steady state. A blend is often characterized by its viscosity ratio, defined as the ratio of the dispersed-phase viscosity to the matrix-phase viscosity. When the viscosity ratio is high, effectively transferring the stresses induced by the mixing process through the matrix phase onto the dispersed phase is difficult. The strategy employed in this dissertation to overcome 
this difficulty is the use of extensional flow fields, which are denoted as strong mixing flows because they are much more efficient at dispersion than are shear flow fields. Extensional flow fields are generated by non-parallel streamlines, for example in geometries which converge in the flow direction. This is especially important in blends with high viscosity ratios, for which pure shear flow fields are unable to breakup dispersed-phase drops due to the rotational aspects of shear flow.

While physical mixing using extensional flow blending is effective in creating fine dispersions, the blends are not necessarily robust to further melt-processing, for example injection molding or profile extrusion of compounded pellets. This is due to the thermodynamic incompatibilities of most polymer systems. Most binary polymer combinations are immiscible with one another, and their blending results in the creation of a two-phase microstructure. This morphology is the result of the dispersive effects of drop deformation and breakup and the coarsening effects of drop coalescence, both of which are influenced by material and interfacial properties and the processing history of the blend. Thermodynamic incompatibility is typically addressed using the addition of a compatibilizer which modifies the polymer-polymer interface. In industry, this may be accomplished using low-molecular weight surfactants, block copolymers, or even reactive compatibilizers, and all of these can be very effective. A relatively new approach that is further explored in this work is the use of a solid phase with surface properties such that it selectively localizes at the polymer-polymer interface. In low-molecular weight colloidal systems, such as oil-in-water emulsions, the resulting systems are called Pickering-Ramsden particle-stabilized emulsions. In this dissertation, nanoparticles with various surface treatments were investigated in an effort to modify the interface and stabilize the fine morphologies produced using extensional flow blending against the coarsening effects of coalescence. In this case, this compatibilization approach is called nanoparticle stabilization.

Extensive research programs in the polymer blending industry typically strive to produce generalized conclusions and relationships that polymer engineers and scientists may apply to their materials and processes effectively and expeditiously. The overall, high-level goal is often to develop processing-structure-properties relationships, as illustrated in Figure 1-1. 
Compounding of materials is usually accomplished using extruders, typically twin-screw extruders (TSES) and sometimes single-screw extruders (SSEs), but the compounded products made must be robust against further melt-processing because these materials are often postprocessed using injection molding, profile extrusion, thermoforming, rotomolding, blow molding, fiber spinning, etc. The processing done to the material imparts a structure, or morphology, within the blend, which in turn directly contributes to the properties that describe how the material will perform in application. In this work, initial compounding strategies are explored, and their efficacy at altering the microstructure of the blends is measured, particularly their effectiveness at dispersing one polymer phase into another. This work is focused on the development of processing-structure relationships, which will inevitably affect the properties and application spaces for the blended material.

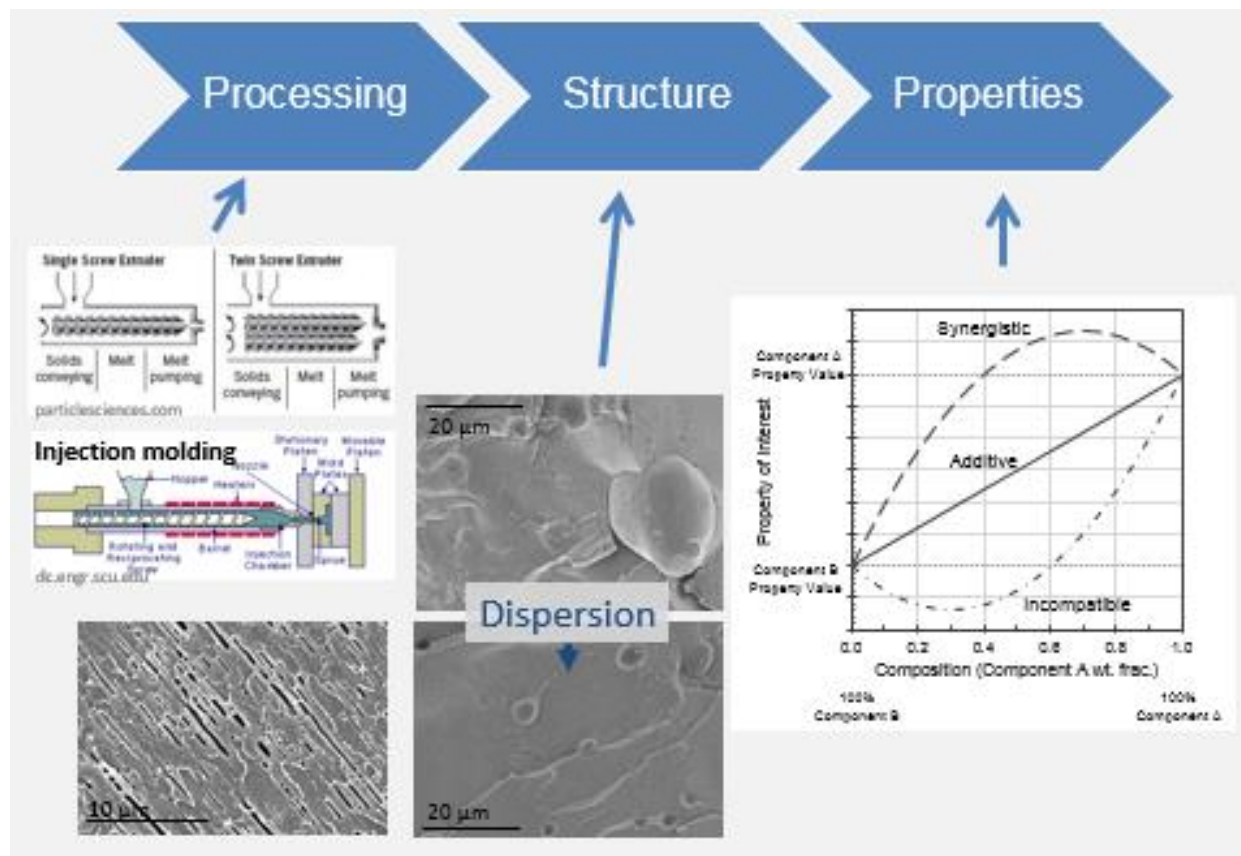

Figure 1-1: High-level goal of research - Processing-Structure-Properties Relationships 


\subsection{Brief Description of Experimental Methods}

The effects of material, process, and geometry variables, such as composition, viscosity ratio, interfacial tension, total extensional strain, extensional strain rate, etc., on the microstructure, or morphology, of immiscible blend systems was investigated in the research presented in this dissertation. Results from mixing experiments incorporating information from existing theories and computations was used to understand general and system-specific processing-structure relationships. Simple, easy-to-fabricate and easy-to-use extensional flow cells (EFCs) were made and their efficacy at altering the morphology of immiscible blends was tested. Furthermore, nanoparticles of various size and surface chemistry were added to blends to stabilize the morphology against coalescence. The conclusions of this work may be used by polymer engineers to easily develop new products with specific properties or even to manufacture existing products with various processing-related advantages. Ultimately, this work offers the following tools that may be used by formulation scientists and manufacturing engineers: 1) The design and proper use of EFCs to impart strong-mixing extensional flow fields onto immiscible polymer blends, 2) formulation and processing guidelines for the incorporation of nanoparticles into binary polymer blends, and 3) the combination of 1) and 2) and knowledge of when each strategy is most advantageous.

A few different polymer blend systems were studied throughout this work in search of identifying a blend system which satisfied the criteria chosen. The selection criteria were that the polymers be immiscible and preferably relatively incompatible and the viscosity ratio be greater than 3.8 for all shear rates expected to occur in the processing operations used. The primary blend systems studied were the following: (1) polycarbonate (PC) dispersed in styreneco-acrylonitrile (SAN), PC/SAN; (2) a high-viscosity grade of polystyrene (PS) dispersed in linear low-density polyethylene (LLDPE), PS/LLDPE; and (3) high-density polyethylene (HDPE) dispersed in a low-viscosity grade of PS, HDPE/PS.

The former two blend systems, PC/SAN and PS/LLDPE, did not fully satisfy the selection criteria, but useful conclusions were found using them and are presented in this dissertation as preliminary results. PC/SAN is a technologically relevant blend that is a successful commercial product offered by polymer material providers, including the funding company Bayer 
MaterialScience, now called Covestro. The grades of PC and SAN chosen had a high viscosity ratio only at high shear rates, and the two polymers, though immiscible, are relatively compatible, and their blending even using shear flow-dominated mixing processes results in blends with fine morphologies, where the dispersed phase is less than $1 \mu \mathrm{m}$ in size. PS and LLDPE are more incompatible thermodynamically, and their blending produces in coarser morphologies from which the effects of the strong extensional flow fields in the blending of this work may be amplified. However, the viscosity ratio of the grades selected was high only for low shear rates.

The HDPE/PS blend system represented a relatively incompatible blend which was satisfactory for the investigation of the efficacy of the blending techniques employed in this work. Furthermore, the blend had a viscosity ratio greater than 3.8 for all shear rates expected to be present in the processes used. The concentration of the dispersed HDPE phase was maintained at $5 \mathrm{wt} \%$, a relatively low concentration to focus the experimentation on the effects of drop deformation and breakup and minimize but not eliminate coalescence. Fumed nanosilica, consisting of spherical, non-porous primary particles usually about $12 \mathrm{~nm}$ in diameter, was employed as the nano-filler to stabilize the morphologies produced by extensional flow blending. Several grades of nanosilica were obtained with various chemical surface treatments which imparted various surface properties on the nano-fillers. Mixing of the nanosilica was investigated first in an internal mixer with various mixing procedures, namely the order of mixing was varied, and the nano-filled blends were also processed using extensional flow blending to identify any coupled effects of extensional flow blending with nanoparticle stabilization.

Several design and processing factors were investigated which govern extensional flow blending. Important geometrical features for the design of extensional flow cells (EFCs) were studied, including the entry profile into a converging section in axisymmetric extensional flow, the total extensional strain, and the number and strength of stretching episodes present in one pass through an EFC. The number of stretching episodes per pass may be increased by adding inserts that nest within the converging cone section of a nozzle, and the strength of these may be varied by varying the gap between the inserts and the converging cone wall where 
components of planar extensional flow are present. Furthermore, both unfilled and nano-filled blends may be processed through the EFCs, the efficacy of extensional flow blending may be characterized for all of the blend types.

\subsection{Objectives}

The objectives of the research described by this dissertation were the following:

1) Quantify the effects of design variables pertinent for extensional flow blending on blend morphology development, and design, build, and test nozzle designs, also called extensional flow cells (EFCs).

Important design variables include blend composition; constitutive rheology characteristics of each phase, including their viscosity ratio; interfacial tension between phases; initial level of dispersion; geometry of EFCs, including number of stretching episodes, total extensional strain, and flow field characteristics of each stretching episode; processing temperature; and flow rate and extensional strain rate.

2) Quantify the effects of design variables pertinent for nanoparticle stabilization on blend morphology development.

Important design variables include blend composition, including loading of nanoparticles; polymer dispersed-phase morphology; nanoparticle morphology, including nanoparticle size, shape, and level of dispersion; wettability of nanoparticles by each polymer phase and wettability parameter for the three-phase blend; processing parameters, including mixer type, temperature, shear rate, mixing time, and order of mixing.

3) Investigate any coupled effects of processing nanoparticle-stabilized blends using extensional flow blending.

While each mixing strategy of 1) and 2) are expected to have significant effects on blend morphology development, coupled effects between 1) and 2) are anticipated to be likely. This would be studied by processing the blends produced in the work toward 2) using the EFCs and processing parameters of 1 ). 


\subsection{Scope}

The scope of this research was to develop general and blend system-specific relationships between processing strategies and parameters and the morphology of the processed blends. These are called processing-structure relationships. This is important because significant alterations of the microstructure of the blend have significant effects on the final properties of the blend, including material, rheological, and mechanical properties. For example, immiscible polymer blends are adept at exploiting the advantageous properties of each polymer while compensating for the weaknesses of each polymer. A very common example of this is given by the typical impact-modified blend in which a tough, often elastomeric polymer is dispersed in a matrix comprised by a strong and stiff, yet brittle polymer. The risk associated with this strategy, however, is that the blend morphology has a significant effect on the properties of the blend. Without processing-structure considerations, since most polymer blends are incompatible, the properties of the blend are often worse than those of each constituent polymer. With appropriate processing, the properties may approach those that would be predicted by a standard rule of mixing theoretical calculation. Finally, with optimized processing and favorable interactions between the polymer phases, synergistic properties may be realized.

The scope of the investigations surrounding nanoparticle stabilization is focused on the production of guidelines that polymer scientists and engineers may use immediately with relatively good confidence. Therefore, commercially available and economically favorable nanoparticles were considered, and the variation of their surface chemistry was done by the material provider and offered in the form of different grades available for purchase. The loading of the nanoparticles was limited to those that would be considered in industry, and reactive chemistries were excluded due to the difficulty in fully understanding the mechanisms underlying their effects. 


\subsection{Significance}

This research described by this dissertation included the development of EFCs intended for use in conjunction with existing mixing devices, such as single-screw and twin-screw extruders, to produce new polymeric material offerings or existing ones using an alternative process. The important distinction between the EFCs of this work and those already existing in industry, such as those shown in Figure 1-2, is that the EFCs designed in this work consist of much simpler and easier to use and understand geometries, translating into a much less expensive and technically prohibitive processing offerings. This is expected to increase their adoption and usefulness in industry.

Furthermore, the incorporation of nanoparticles into the blends represents the creation of novel material systems that may be advantageous to the industry. Generally, adding solid nanoparticles imparts improved stiffness and sometimes strength in polymer systems, and the thermodynamic compatibilization potential of this nanoparticle phase, also called nanoparticle stabilization, has great potential to offer polymer scientists and engineers a route to design and produce new polymeric offerings, especially when nanoparticle stabilization is combined with extensional flow blending through EFCs.

- Y. Suzaka. 1982. Mixing Device. US Patent 4,334,783.

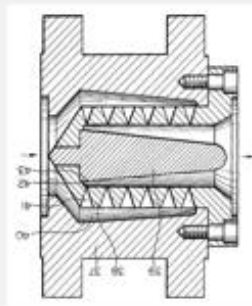

- X.Q. Nguyen and L.A. Utracki. 1995. Extensional Flow Mixer. US Patent $5,451,106$.

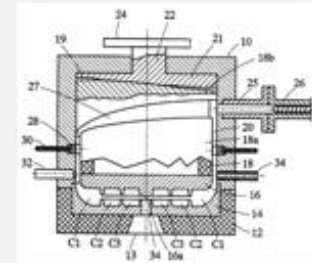

- S.S. Eggen and R. Nygaard. 2001. Mixing Device. US Patent $6,299,342$.

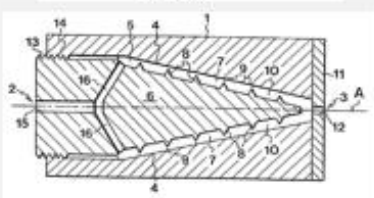

Figure 1-2: Patented extensional flow mixing devices [1-3] 


\section{Background and Theory}

The history of polymer blends began in 1846 when Parkes patented a blend of natural rubber and gutta percha [4]. Much of the beginning of polymer understanding in the beginning of the $20^{\text {th }}$ century was centered around cellulose-based polymers and rubbers. Development of most of today's main commercial polymers took place during World War II, and plastics began to replace traditional materials in multitudes of applications throughout the latter half of the $20^{\text {th }}$ century, bringing us into the Age of Plastics. By the 1980 s and 1990 s, blending and compounding became important. Contemporary polymer scientists and engineers typically solve problems and develop new products based on blending existing polymers rather than synthesizing new polymer chemistries. Much of the aim today is the development of fundamental processing-structure-properties relationships to facilitate the design and application of blended products.

In this chapter, the background and theory of polymer blending will be discussed. An in depth analysis of the theory dictating morphology development in polymer blending will be given. Finally, the effects of addition of nanoparticles to polymer blends will be described.

\subsection{Polymer Blending}

Polymer blending is an important technique for tailoring the properties of a polymer material without the capital expenditure and learning curve associated with the production of new polymer chemistries. Since most polymers are immiscible with one another, their blending usually results in two-phase systems. The properties of the final blend are dependent on the properties and loading of the constituent materials, the properties of the interface between the polymers, and the microstructure, or morphology, of the blend [5]. The potential results of blending on one of the final properties of the blend are summarized in Figure 2-1. Since most polymers are not only immiscible with others but also incompatible with others, blending without robust means for physical and thermodynamic considerations often yields property values less desirable than those of the constituent polymers. This corresponds to the incompatible curve of Figure 2-1. Alternatively, if the morphology and other deciding factors 
are appropriate, then an additive rule of mixtures may be used to predict the blend property value based on the concentration of each component. Alternatively, the ideal situation is that blending of two constituent materials produces blends with property values superior to those of each individual phase. This is shown in Figure 2-1 by the synergistic curve. Also shown is that the effect of blending on the properties of interest may also depend on composition.

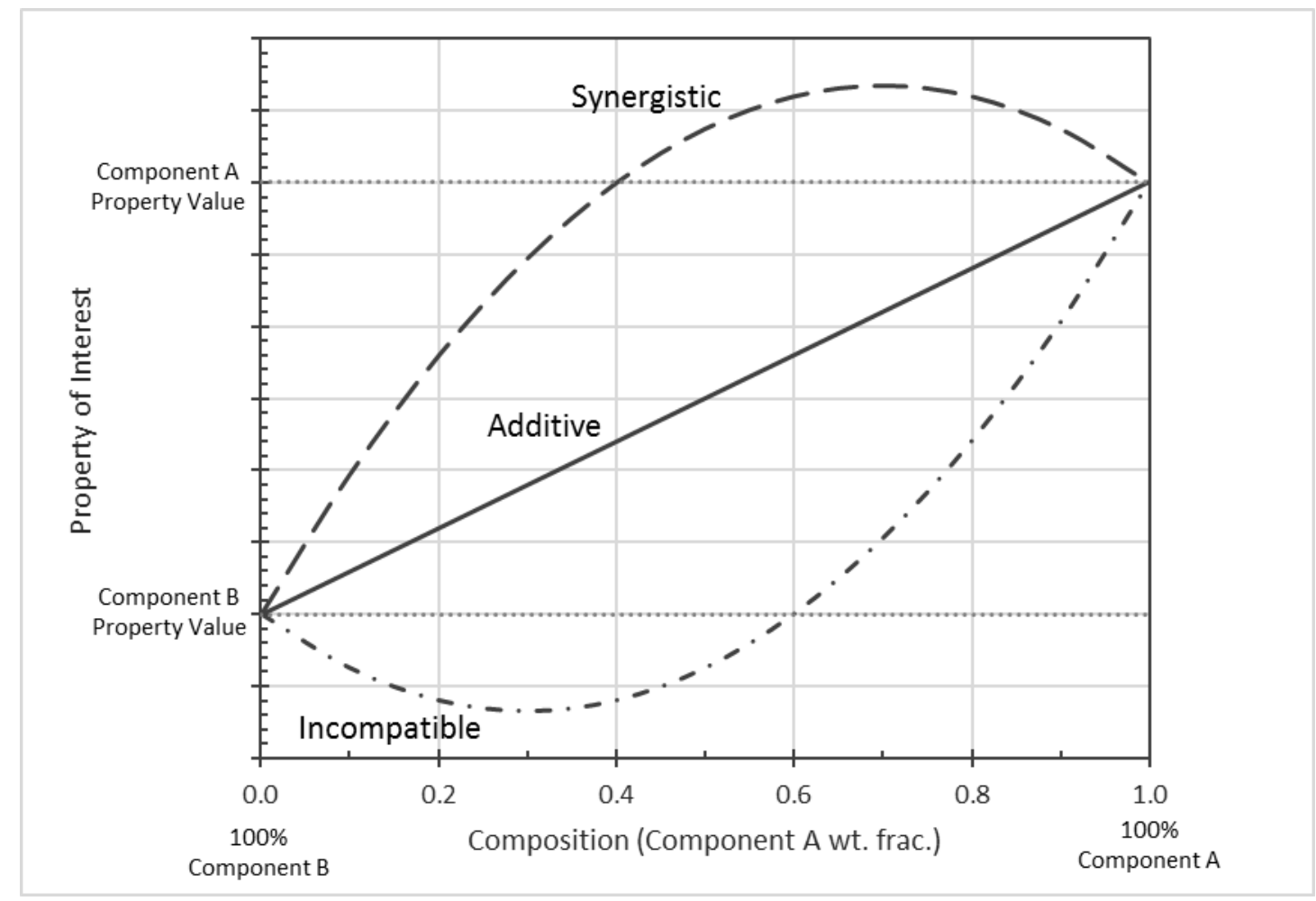

Figure 2-1: Polymer blending potential effects on properties

\subsubsection{Thermodynamics of Polymer Blends}

For a polymer to dissolve in a matrix (a solvent or another polymer), the Gibbs free energy of the system must decrease by having the polymer dissolve rather than remain as a distinct phase [6]:

$$
\Delta G_{\text {mix }}=\Delta H_{\text {mix }}-T \Delta S_{\text {mix }}<0 .
$$

For most polymers, the enthalpy change on mixing is positive; therefore, the change in entropy on mixing must be sufficiently positive for dissolution to occur. While Equation (2-1) is a 
necessary criterion for miscibility, it is not the only one. The system must also be thermodynamically stable. To this end, the partial molar Gibbs free energy of the mixture, $\Delta g_{\mathrm{m}}$, is important, given by

$$
\Delta g_{\mathrm{m}}=x_{1} \Delta \mu_{1}+x_{2} \Delta \mu_{2}
$$

where $x_{1}$ and $x_{2}$ are the mole fractions of Component 1 and Component 2, such that $x_{1}+x_{2}=1$, and $\Delta \mu_{i}=\mu_{i}-\mu_{i}{ }^{0}$, where $\mu_{i}$ is the partial molar Gibbs free energy, or chemical potential, of each component in the mixture and $\mu_{i}^{0}$ is the chemical potential of each pure component.

Differentiating Equation (2-2) with respect to $x_{2}$ and applying the Gibbs-Duhem equation for partial molar quantities, in this case $\sum_{i} x_{i} d \mu_{i}=0$, yields:

$$
\frac{d \Delta g_{\mathrm{m}}}{d x_{2}}=\Delta \mu_{2}-\Delta \mu_{1} .
$$

It is possible to plot $\Delta g_{\mathrm{m}}$ vs. $x_{2}$ at various temperatures. Simultaneously solving Equations (2-2) and (2-3) allows one to compute the chemical potential of each phase using this plot. Examining the plot, one also finds that at relatively lower temperatures, two different compositions may yield the same set of $\Delta \mu_{1}$ and $\Delta \mu_{2}$. This means that it is possible for two liquid phases to be in equilibrium (that is to have the same chemical potential for each component) where one phase has composition $x_{2}{ }^{\prime}$ that yields $\Delta \mu_{1}$ and $\Delta \mu_{2}$ and the other phase has composition $x_{2}{ }^{\prime \prime}$ that yields the same. These compositions $x_{2}{ }^{\prime}$ and $x_{2}{ }^{\prime \prime}$ are called binodal points. Compiling them on a plot of $T$ vs. $x_{2}$ yields the binodal curve, which has a maximum at the upper critical solution temperature (UCST), above which the components are miscible and only one phase is formed, until a lower critical solution temperature (LCST) is reached.

Also from the plot of $\Delta g_{\mathrm{m}}$ vs. $x_{2}$, one notices that between binodal points the curve changes from concave upward to concave downward. From stability analysis, the inflection point on each side of the concave downward section is a cut-off for stability, in that compositions deviating from $x_{2}^{\prime}$ or $x_{2}^{\prime \prime}$ may remain in a metastable miscible phase until the composition reaches the inflection point, called a spinodal point, at which the solution spontaneously separates to a two-phase immiscible system. Similar to the binodal curve, the locus of the spinodal points on a $T$ vs. $x_{2}$ plot is called the spinodal curve. Within this envelope, 
only an immiscible two-phase system is possible. The stability of the system is the second criterion for miscibility, in addition to Equation (2-1).

\subsubsection{Kinetics during Processing of Blends}

The kinetics of polymer melt processing, including that of blends, is well studied and will not be belabored in this dissertation. The general equations dictating fluid dynamics are the Equation of Continuity and the Equations of Motion. These describe the physics of the flow fields present in polymer processing operations. The Equations of Motion may be simplified assuming incompressible, Newtonian flow, to the form of the Navier-Stokes Equations. The inherent rheological properties are also governing factors over the behavior of the system, and these are described by the Constitutive Equations of the polymer phases. The simplest constitutive equation is that for Newtonian flow, in which the fluid's resistance to flow, called viscosity, $\eta$, is constant with respect to the flows:

$$
\eta=\eta_{0}
$$

where $\eta_{0}$ is the Newtonian shear viscosity, which is usually observed at low shear rates of flow for typical polymer melts in the region called the zero-shear plateau. Polymer melt viscosity typically depends strongly on shear rate and exhibits shear-thinning behavior at intermediate to high shear rates. In this region, the constitutive equation that dictates the shear-thinning flow is that for Power-Law flow:

$$
\eta=K \dot{\gamma}^{n-1},
$$

where $K$ is the consistency metric, $j$ is shear rate, and $n$ is the power-law index, which ranges from 0-1 for shear-thinning flow. Many other constitutive equations have been postulated and are useful in describing the specifics of distinct polymer flows, but these are typically associated with greatly increased complexity.

Fluid flow may generally be split into two regimes: Shear flow and extensional flow. In shear flow, flow streamlines are parallel, and in extensional flow streamlines are not parallel and either converge or diverge. Flow may be drag-flow driven, where the molten polymer flows due to stress transfer imparted onto it by moving surfaces, for example in a screw extruder. Flow may also be pressure-driven, where material flows from high-pressure zones to 
low-pressure areas, for example at the die head at the exit of an extruder. Simple schematics of examples of shear and extensional flows are given in Figure 2-2. The geometry of the equipment used in the polymer processing is paramount in dictating the types of flow fields imparted onto the systems. Generally, real processes have contributions of both shear and extensional flows and shear stresses and effects of pressure differentials are present.

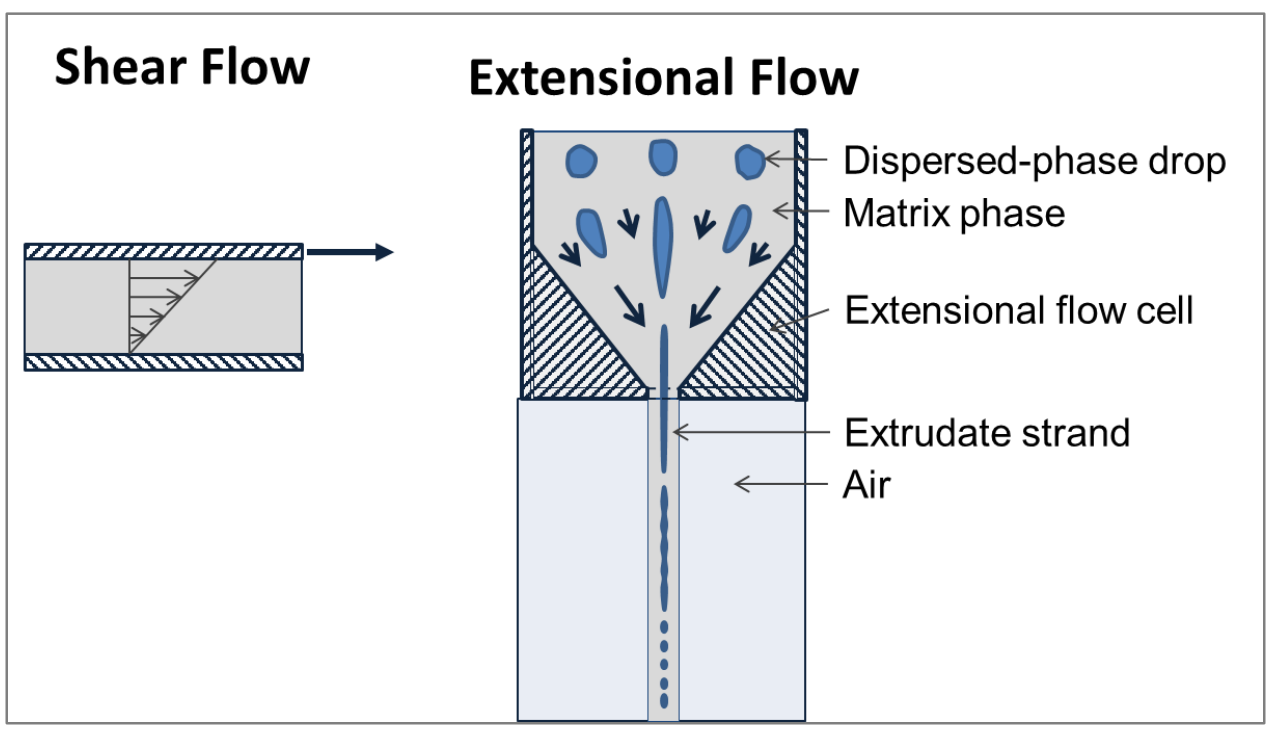

Figure 2-2: Shear flow and extensional flow field schematics. Left - Sliding-plate drag flowdriven shear flow. Right - Extensional, pressure-driven flow in a converging nozzle along with schematics of the deformation and breakup of a dispersed polymer phase.

The overall physics of the processing operations are often described by dimensionless numbers, which compare the relative magnitudes of various effects to one another. For example, the Reynolds number, Re, is likely the most commonly used dimensionless number in fluid dynamics, and it compares the contributions of inertial effects to viscous effects. This typically approaches zero for polymer flows due to the very high viscosities of polymers, and this type of flow is called creeping flow. Body forces like gravity and electromagnetic effects are also important in many blends, but for this work, they are negligible compared to viscous and other effects. At the length scales associated with typical dispersed phase domains, the effects of surface, or interfacial, tension become important. The capillary number, $\mathrm{Ca}$, compares viscous effects to interfacial tension effects, and it is regarded as the most important dimensionless group that is useful in describing the behavior of polymer blending. The viscosity 
ratio, $p$, which is the ratio of the dispersed-phase viscosity to that of the matrix phase is also very important. Other groups investigated in this work include the Deborah number, De, which compares the polymer relaxation time to the characteristic time scale of the process, and the Weissenberg number, Wi, which compares the relaxation time to the time scale associated with the strain rate of the flow. The important dimensionless groups are discussed further throughout this dissertation.

\subsubsection{Mixing Strategies}

The mixing of distinct material phases into a polymer matrix is usually accomplished in extruders, either single-screw extruders (SSEs) or twin-screw extruders (TSEs). SSEs are very efficient at generating pressure and stable exit flow rates, while TSEs are more adept at compounding operations. TSEs may be counter- or co-rotating, with co-rotating being more popular and efficient at passing material back and forth between the screws and effectively distributing and mixing dispersed phases in the blends. The screw design of extruders is the most effective way to dictate the flow fields within the extruder and tune the mixing intensity. For example, kneading blocks are very efficient at dispersing materials in the blends due the relatively higher degree of extensional flow fields present in those sections. Most of these implementations then rely on drag flow to improve mixing efficiency. Pressure-driven flows may also be utilized to do the same thing. Static mixing elements may be added at the die exit of the extruder, where the molten material is forced to flow through geometries that either disperse or distribute the phases. This approach is the one taken in this work, in which dispersive static mixing elements, or cells, or nozzles, are designed with converging geometries that impart strong mixing extensional flow fields onto the blend. Static mixers utilize pressuredriven flows generated by SSEs, TSEs with gear pumps, and injection molding machines. They are used in industry often in the form of distributive mixing elements to effectively distribute solid or liquid dispersed phases into polymer matrices. The extensional-flow static mixing elements designed and tested in this work focus on dispersive mixing, and the only distributive mixing contributions are in the direction of flow, with little distributive mixing normal to the direction of flow and with little re-orientations of flow. 
While the physical blending operations are critical in affecting the level of mixing of composite material systems, thermodynamics are critical as well. Most polymers are immiscible with one another and form separate, distinct phases upon their blending with an interface between them with an associated interfacial tension. The relative magnitude of this interfacial tension has a large effect on the behavior of the blend system, with the size of the dispersed phase directly proportional to the interfacial tension. To combat this, compatibilization efforts are taken to modify the interface between the polymers. Surfaceactive agents, or surfactants, are low-molecular weight molecules that have polar and nonpolar ends that are adept at modifying the interface. Block copolymers are also used which work by the same mechanism, and reactive compatibilizers may also be used to covalently bond the phases together.

\subsection{Morphology Development}

\subsubsection{Description of Morphologies}

Given that most polymers are immiscible with one another, mixing them usually results in the formation of microstructures made up of distinct phases. The type of microstructure, or morphology, formed depends on the volume fractions of each phase, the interfacial tension between phases, and processing. Several morphology types may be formed, including but not limited to those shown in Figure 2-3. 


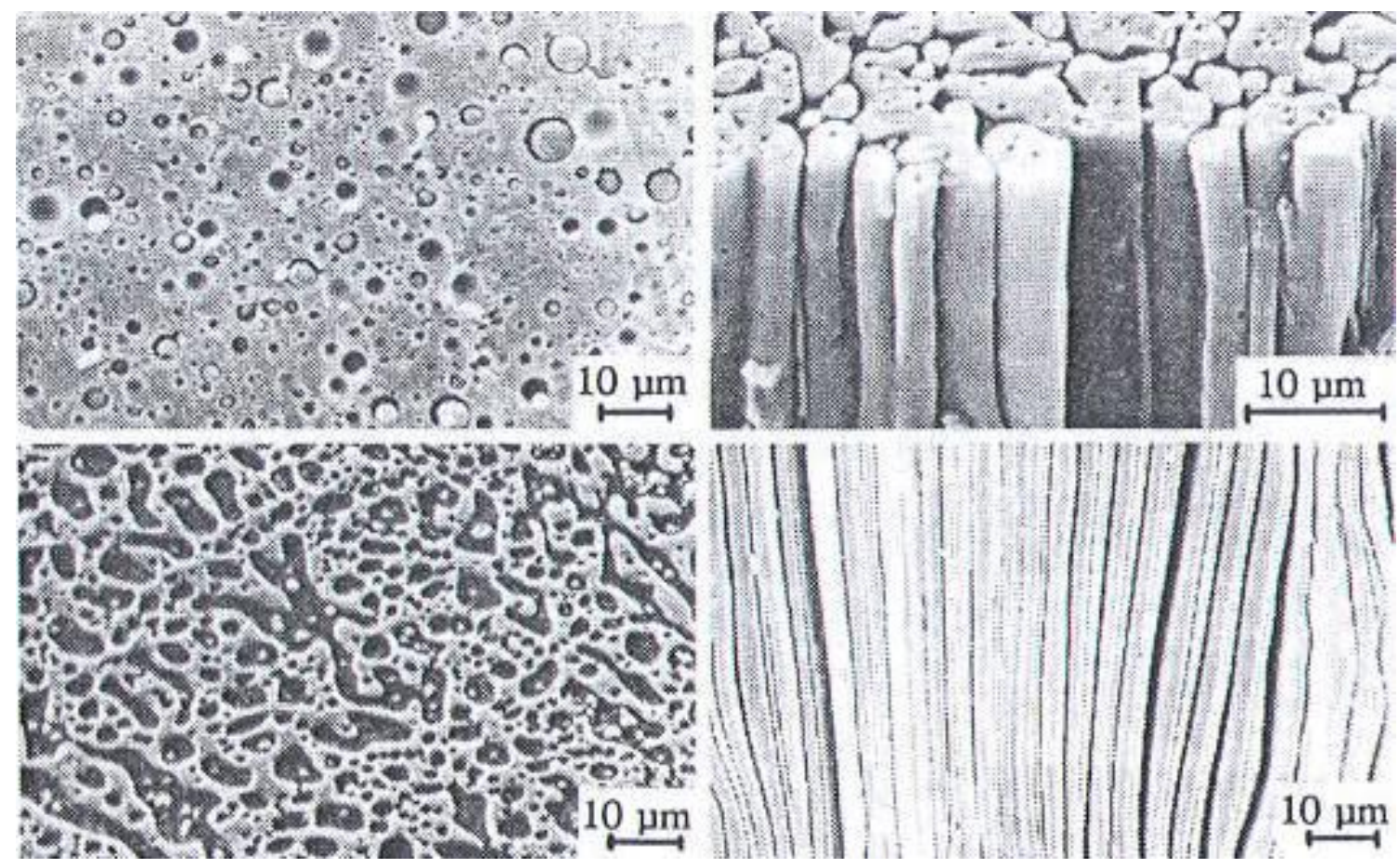

Figure 2-3: Two-phase morphologies (Reproduced with permission from Meijer et al. 1988

[7]). Top-left, dispersed, or drops-in-matrix, morphology; top-right, fibrillary morphology; bottom-left, cocontinuous morphology; bottom-right, lamellar morphology.

The drop-in-matrix morphology is the most common morphology encountered in commercial polymer blends. Quantification of the level of mixing of drop-in-matrix blends is given by the dispersion and distribution of the dispersed phase. Dispersion is defined by the decrease in length scale of the dispersed-phase drops, where fine dispersions are given by blends with fine, small-diameter drops. Concomitantly, the overall interfacial surface area and the ratio of interfacial area to volume increase with increasing dispersion. Very fine dispersions are typically indicative of relatively compatible blends, and dispersed-phase drop sizes of $1 \mu \mathrm{m}$ or less are often desired. However, the blend property of interest influences the optimum drop size greatly. For example, for impact resistance, the mechanism for impact absorption of the material dictate whether very small or relatively larger dispersed phase drops are desired. Distribution is defined by the relative spacing between drops, where increasing distribution yields blends with more evenly arranged drops throughout the matrix. The distribution may be quantified in several ways, with interparticle spacing one of the common metrics. 


\subsubsection{Drop Deformation}

Since the viscosity of liquid polymers in solution or in the molten state is usually very high, the Reynolds number of flow during processing is usually very low and inertial effects may be ignored compared to viscous effects. Furthermore, the densities of liquid polymers usually does not vary greatly (low Bond numbers) and density and buoyancy effects may also be ignored. The important forces that govern the behavior of liquid polymer systems then are characterized by the capillary number, $\mathrm{Ca}$, which is the ratio of viscous stresses, which deform the dispersed phase, and interfacial stresses, which conserve the shape of the dispersed phase:

$$
\mathrm{Ca}=\frac{\tau R}{\sigma_{12}},
$$

where $\tau$ is the viscous stress resulting from flow of the matrix material and $\sigma_{12} / R$ is the interfacial stress due to interfacial tension, $\sigma_{12}$, acting on a dispersed-phase drop of radius $R$. Flow fields may be characterized by the components of shear flow and extensional flow.

The capillary numbers for simple shear flow fields and for extensional flow fields are

$$
\begin{gathered}
\mathrm{Ca}_{\mathrm{s}}=\frac{\eta_{\mathrm{m}} \dot{\gamma} R}{\sigma_{12}} \\
\text { and } \mathrm{Ca}_{\mathrm{e}}=\frac{\eta_{\mathrm{e}, \mathrm{m}} \dot{\varepsilon} R}{\sigma_{12}},
\end{gathered}
$$

respectively, where $\eta_{\mathrm{m}}$ and $\dot{\gamma}$ are the matrix-phase shear viscosity and shear rate and $\eta_{\mathrm{e}, \mathrm{m}}$ and $\dot{\varepsilon}$ are the matrix-phase extensional viscosity and extensional strain rate.

Using Equation (2-6) to characterize blending of immiscible polymers, typically the dispersed phase is initially coarse, with large $R$ and large Ca. Viscous stresses outweigh interfacial stresses, and dispersed drops stretch and break up. With time, drop size decreases, thus Ca decreases, until a point is reached at which interfacial stresses become significant and breakup no longer occurs. For Ca below this critical point, a steady ellipsoidal drop shape is formed. The critical point, $\mathrm{Ca}_{\text {crit, }}$ has been determined experimentally under quasi-equilibrium conditions by slowly increasing the rate of flow and evaluating the long-term stability of deformed drops, and it is known to depend on the type of flow and the viscosity ratio, $p$ :

$$
p=\frac{\eta_{\mathrm{d}}}{\eta_{\mathrm{m}}},
$$


where $\eta_{\mathrm{d}}$ is the dispersed-phase viscosity [8-9]. The dependence of $\mathrm{Ca}_{\text {crit }}$ on the type of flow and viscosity ratio is shown in Figure 2-4; this is called the "Grace curve" in the literature. The assumptions and experimental conditions used to create the Cacrit curves are described in the figure's caption. Above the curve, breakup may occur, and below the curve, a stable drop size and shape is assumed and breakup is not predicted. The $\mathrm{Ca}_{\text {crit }}$ vs. $p$ curve depends on the flow field. The curves for simple shear and planar extensional (2-D elongation) flows are plotted in Figure 2-4. Other flow fields and mixed flow fields have curves that fall between those two. Of particular interest is the asymptote that is approached at $p=3.8$ for simple shear flows; this will be discussed further below.

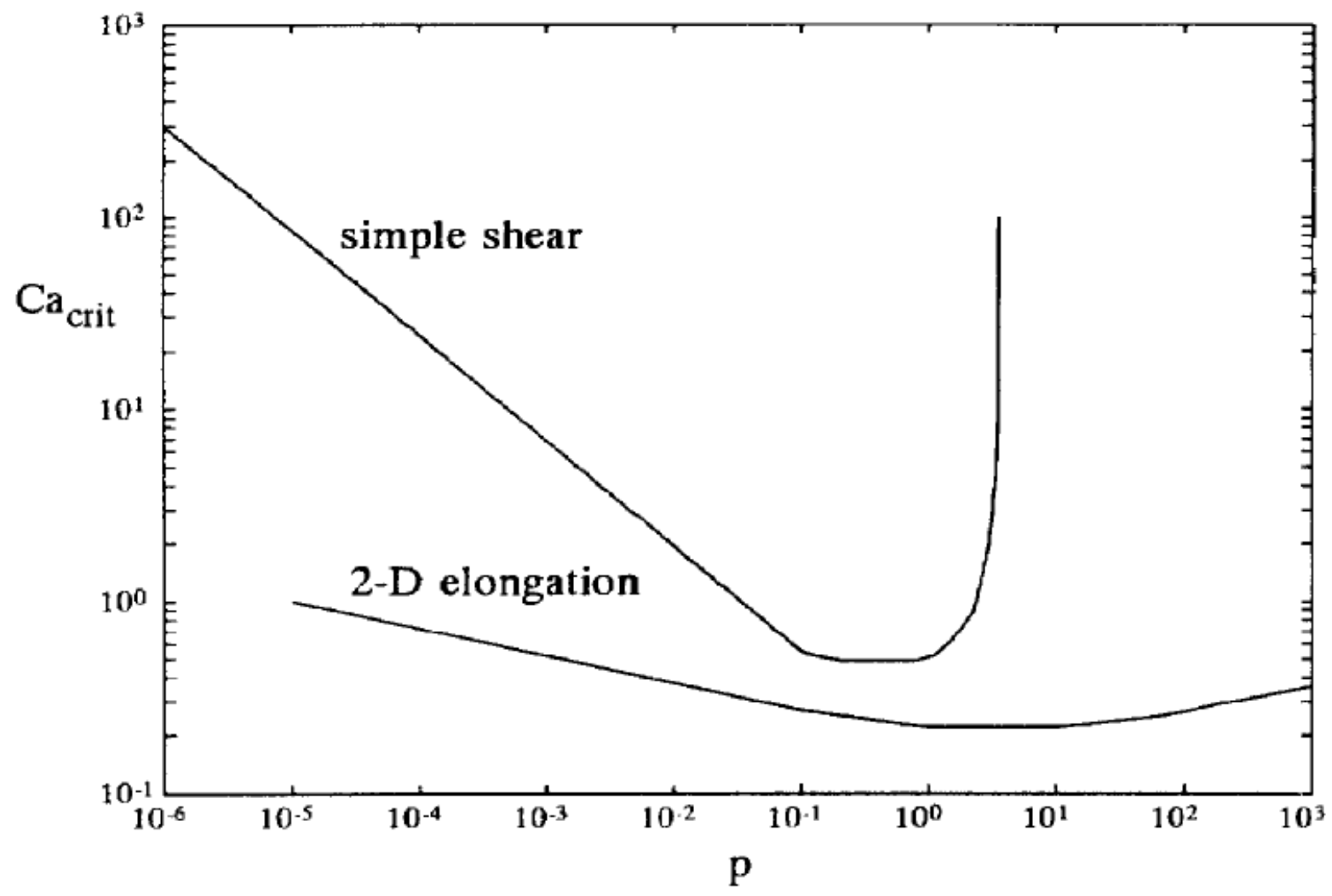

Figure 2-4: Critical capillary number as a function of flow type $\&$ viscosity ratio (Reproduced with permission from Janssen \& Meijer, J. Rheol., 37, 597-608, 1993 [10]. Copyright 1993, The Society of Rheology). Cacrit for breakup of one, initially spherical, Newtonian drop in quasisteady, well-defined flow of Newtonian matrix. 
Due to flow, initially spherical drops stretch into elongated ellipsoids, as shown in Figure 2-5. Drop deformation, $D$, may be quantified by

$$
D=\frac{L-B}{L+B},
$$

in which $L$ and $B$ are the length and width, respectively, of the deformed ellipsoid with circular cross section. Affine deformation may be described by an affine transformation, composed of a linear transformation, e.g. rotation, shear, extension, or compression, and a rigid body translation. This deformation occurs when $\mathrm{Ca}$ is sufficiently larger than $\mathrm{Ca}_{\text {crit, }}$ e.g. Ca 2 to 10 times greater than $\mathrm{Ca}_{\text {crit }}[11]$. In simple shear flow, affine deformation results in

$$
D=\frac{\gamma}{\sqrt{4+\gamma^{2}}}
$$

where $\gamma$ is total shear, $\gamma=j t$, in which $t$ is time, and the orientation angle, $\varphi$, between the drop length axis and the flow streamlines is given by

$$
\tan \varphi=\left(\frac{1}{2} \gamma+\frac{1}{2} \sqrt{4+\gamma^{2}}\right)^{-1} .
$$

The drop initially stretches at a $45^{\circ}$ angle with respect to the flow stream lines and rotates toward the streamlines as $\gamma$ increases. The rotational aspect of shear flow defines it as a weak flow, i.e. rotational flow. This is the source of the asymptote in the Grace curve, Figure 2-4, at $p$ = 3.8 for simple shear flow, meaning initially spherical drops cannot affinely deform in simple shear flow when the viscosity ratio is greater than 3.8 , rather they rotate and assume a steady ellipsoidal shape. Affine deformation in extensional flow results in the drop extending exponentially in the principal direction of strain; for planar (2D) extensional flow,

$$
D=\frac{e^{2 \varepsilon}-1}{e^{2 \varepsilon}+1},
$$

where $\varepsilon$ is the total extensional strain, $\varepsilon=\dot{\varepsilon}$. It is evident, therefore, that for affine deformation, $D$ depends only on total shear or total extensional strain, regardless of the combination of rate and time to get there. 


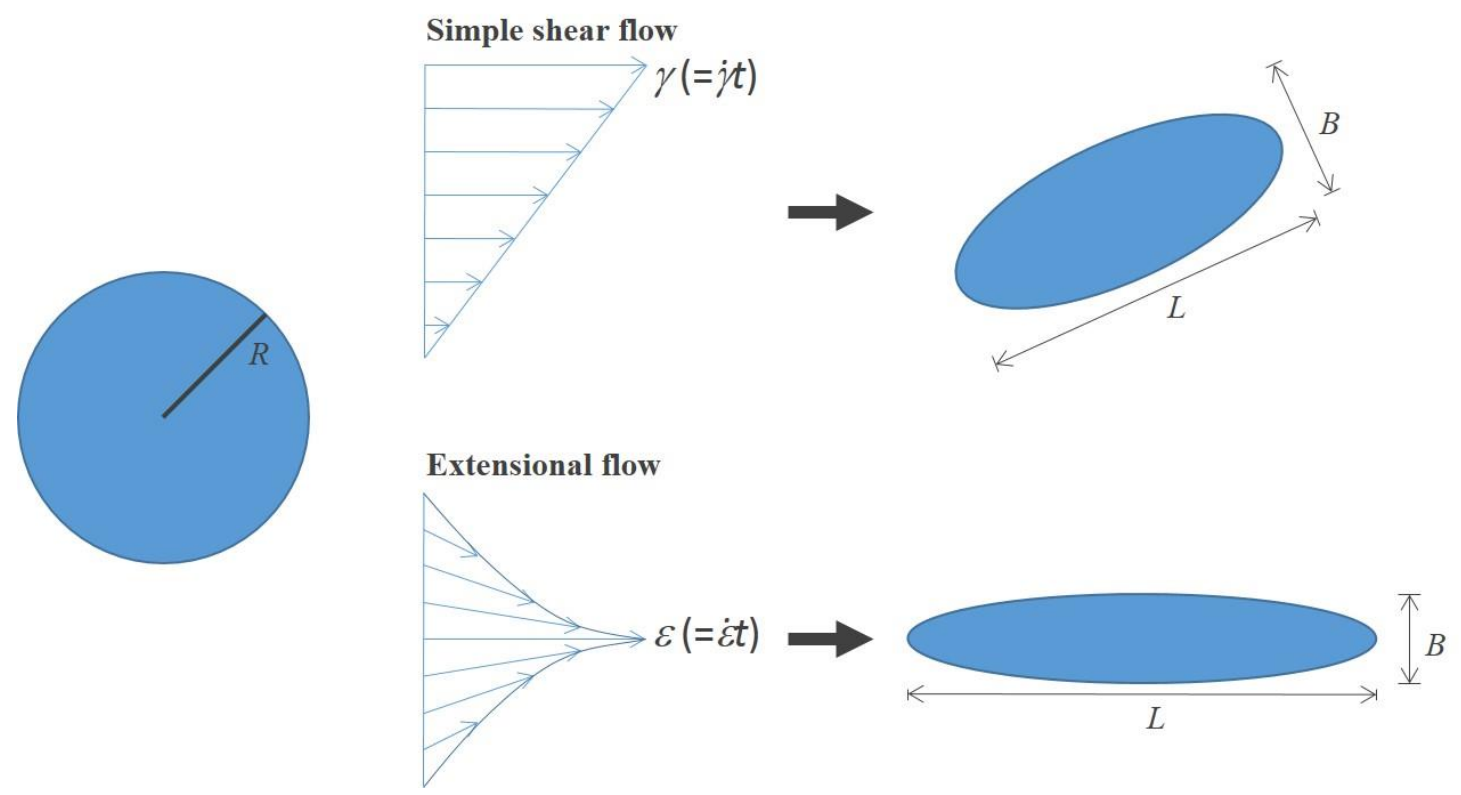

Figure 2-5: Drop deformation in different types of flow. Schematic representation of affine deformation of initially spherical drop in simple shear and extensional flow.

Simple shear flow is also results in flattening of the extending drops; one cross-sectional axis is compressed while the other does not change. However, interfacial tension limits this when the curvature at the edges gets large enough, and the drop width no longer deforms affinely. With the drop length still stretching affinely, assuming a circular cross section and incompressibility yields

$$
D=\frac{\left(\frac{1}{2} \gamma+\frac{1}{2} \sqrt{4+\gamma^{2}}\right)^{3 / 2}-1}{\left(\frac{1}{2} \gamma+\frac{1}{2} \sqrt{4+\gamma^{2}}\right)^{3 / 2}+1}
$$

in simple shear flow, which for large shear values $(\gamma>5)$ may be approximated by

$$
D=\frac{\gamma^{3 / 2}-1}{\gamma^{3 / 2}+1} \text {. }
$$

Similarly, planar (2D) extensional flow also causes flattening, and again assuming interfacial tension maintains a circular cross section yields

$$
D=\frac{e^{3 \varepsilon / 2}-1}{e^{3 \varepsilon / 2}+1} .
$$


The assumption that interfacial tension maintains a circular cross section in Equations (2-14) and (2-16) results in a slower growth of $D$ compared to affine deformation with flattening, Equations (2-11) and (2-13). In all cases, though, extensional flow (exponential) results in much faster deformation than shear flow (linear). In practice, however, extensional flow fields are usually much shorter lived; contractions are inherently finite for flow to occur.

\subsubsection{Breakup}

When drops are highly elongated into threads, the capillary number Ca decreases as thread radius decreases, as per Equation (2-6), where $R$ then is the thread radius; therefore, interfacial stresses become significant. Small disturbances of the interface grow in amplitude due to interfacial tension trying to minimize interfacial area until breakup occurs and the thread breaks up into a line of small droplets.

\subsubsection{Breakup of Newtonian Thread in Quiescent Newtonian Matrix}

The breakup of jets was first studied by Lord Rayleigh [12] for the breakup of water jets, including surface tension and inertia, in air. Later, the viscosity of the threads was included [13], then Tomotika [14] added the viscosity of the matrix and the effect of viscosity ratio. Small sinusoidal disturbances, Rayleigh disturbances, are imposed the interface between a liquid thread and the matrix fluid, both without flow, as in Figure 2-6; these are given by

$$
R(z)=\bar{R}+\alpha \sin \left(\frac{2 \pi z}{\lambda}\right),
$$

where $\alpha$ and $\lambda$ are the amplitude and wavelength of the disturbance, respectively, and $\bar{R}$ is the average radius (smaller than the initial radius $R_{0}$ ):

$$
\bar{R}=\sqrt{R_{0}^{2}-\frac{\alpha^{2}}{2}} .
$$




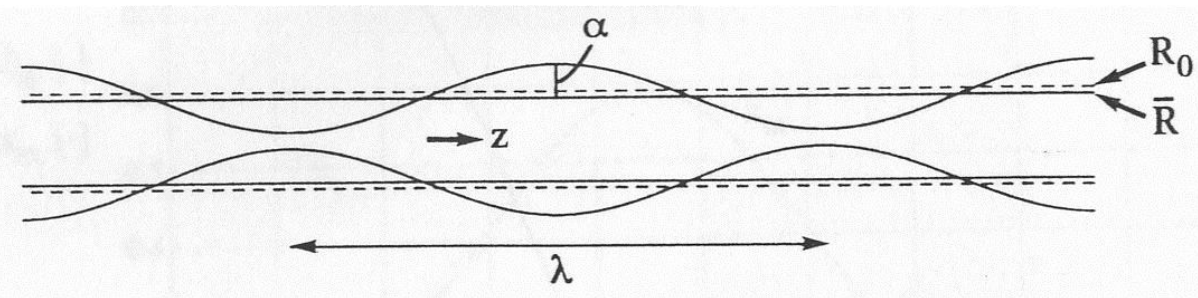

Figure 2-6: Sinusoidal disturbance on thread surface (Reproduced with permission from Janssen 1993 [11]). Disturbance with amplitude $\alpha$ and wavelength $\lambda$ on the surface of a thread of initial radius $R_{0}$ and average radius after disturbance of $\bar{R}$.

For disturbances with wavelength larger than the initial thread circumference, $\lambda>2 \pi R_{0}$, interfacial area decreases monotonically with increasing disturbance amplitude; therefore, these disturbances grow with time. Conversely, disturbances with wavelengths smaller than the initial thread circumference initially increase interfacial area as amplitude grows, and these disturbances damp with time. Assuming the disturbance amplitude $\alpha$ grows exponentially with time and substituting the dimensionless wave number $x$ for wavelength $\lambda, x=2 \pi R_{0} / \lambda$, result in

where the growth rate $q$ is

$$
\alpha=\alpha_{0} e^{q t}
$$

$$
q=\frac{\sigma_{12} \Omega(x, p)}{2 \eta_{\mathrm{m}} R_{0}},
$$

which depends on the dimensionless growth rate of the disturbance $\Omega$, a function of wave number and viscosity ratio. Initially, very small disturbances of all wave numbers are present, but for a given $p$, whichever wave number $x$ yields the largest growth rate $\Omega$ becomes dominant, i.e. a dominant wave number $x_{m}$ and dominant growth rate $\Omega_{m}$ exist, given in Figure 2-7. The dominant disturbance amplitude grows until it equals the average radius, i.e. $\alpha=\bar{R}$, at which point the thread breaks up into a line of small droplets. The breakup amplitude $\alpha_{\mathrm{b}}$ and final droplet radius $R_{\text {droplets }}$ (for incompressible liquids) are

$$
\begin{gathered}
\alpha_{\mathrm{b}}=\sqrt{2 / 3} R_{0} \\
\text { and } R_{\text {droplets }}=R_{0}\left(\frac{3 \pi}{2 x_{\mathrm{m}}}\right)^{1 / 3},
\end{gathered}
$$

respectively. 


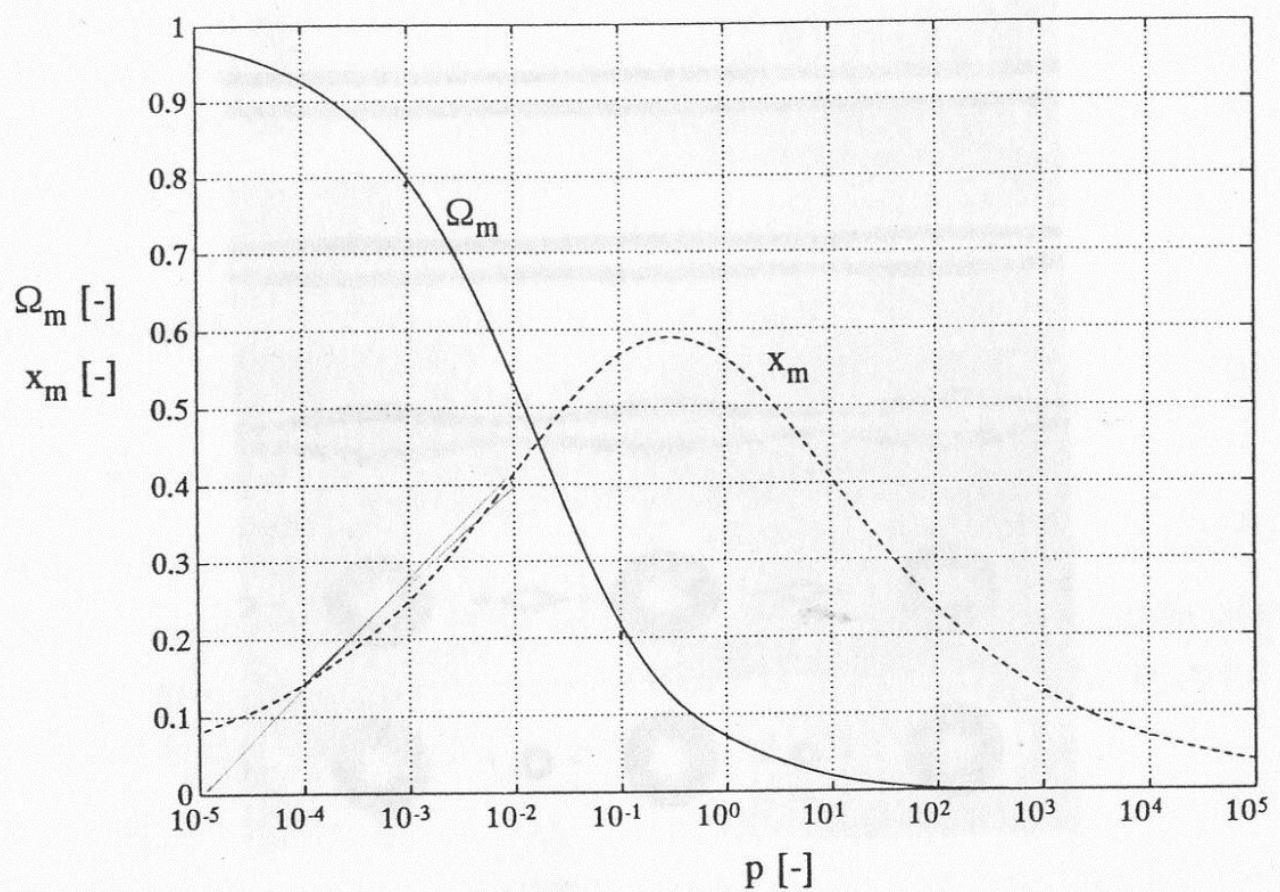

Figure 2-7: Dominant growth rate $\&$ wave number vs. viscosity ratio (Reproduced with permission from Janssen 1993 [11]). $\Omega_{\mathrm{m}} \equiv$ Dominant growth rate; $x_{\mathrm{m}} \equiv$ wave number of interfacial disturbances; and $p \equiv$ viscosity ratio.

Given the growth rate in Equation (2-19), the time required for breakup, $t_{b}$, of the thread is given by

$$
t_{\mathrm{b}}=\frac{1}{q} \ln \left(\frac{\alpha_{\mathrm{b}}}{\alpha_{0}}\right) \text {. }
$$

The initial amplitude $\alpha_{0}$ has been estimated based on Brownian motion due to temperature fluctuation [15]:

$$
\alpha_{0}=\sqrt{\frac{21 k T}{8 \pi^{3 / 2} \sigma_{12}}},
$$

where $k$ is Boltzmann's constant. This prediction yields $\alpha_{0} \approx 1 \mathrm{~nm}$. Combining Equations (2-20), (2-21), (2-23), and (2-24) yields the following expression for the breakup time of a thread in a stationary matrix:

$$
t_{\mathrm{b}}=\frac{\eta_{\mathrm{m}} R_{0}}{\sigma_{12} \Omega_{\mathrm{m}}} \ln \left(\frac{10^{23} \sigma_{12} R_{0}^{2}}{T}\right)
$$


Extrapolation of Tomotika's small-amplitude linear analysis [14] all the way to breakup of the thread has been verified numerically by Janssen [11].

Experimental studies of the breakup of a Newtonian thread in a quiescent Newtonian matrix match the linear theory by Tomotika [14] well, e.g. that shown in Figure 2-8. This technique has even been used to measure interfacial tension, called the breaking thread method. Given the viscosity of each phase, measurements of $\ln \alpha$ vs. time allow the calculation of the growth rate $q$; then, assuming $\Omega=\Omega_{\mathrm{m}}$ from Figure 2-7, the interfacial tension $\sigma$ may be calculated using Equation (2-20). Also notable from Figure 2-8 is the formation of smaller satellite droplets between the main drops. These form during the last stage of breakup due to very fast growth of Rayleigh disturbances on the fine filament between the main drops, and in general, for larger viscosity ratio, fewer but larger satellite droplets are formed due to the viscous damping of internal flows [11].

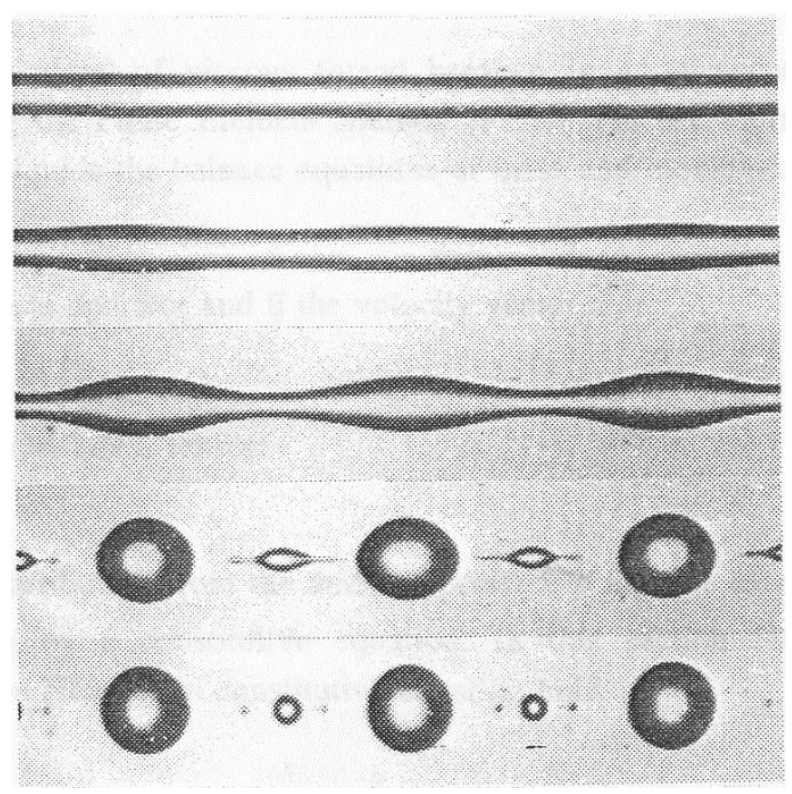

Figure 2-8: Breakup of a Newtonian thread in a quiescent Newtonian matrix (Reproduced with permission from Janssen 1993 [11]). Thread: castor oil, $\eta_{\mathrm{d}}=0.74 \mathrm{~Pa} \cdot \mathrm{s}, R_{0}=0.35 \mathrm{~mm}$; matrix = silicone oil, $\eta_{\mathrm{m}}=0.94 \mathrm{~Pa} \cdot \mathrm{s} ; \sigma_{12}=4.1 \mathrm{mN} / \mathrm{m}$; photographs after every second. 


\subsubsection{Breakup of Viscoelastic Thread in a Quiescent Matrix}

Breakup of a viscoelastic thread in a quiescent matrix is a more complex problem. The breaking thread method has been used successfully for polymer melts since the deformation rates are small, but this only valid if the relaxation of stresses that were induced during the formation of the thread takes much longer than the relaxation times of the polymers. For viscoelastic threads, elongational viscosity may increase dramatically during the breakup process due to strain hardening, which slows the breakup process. The disturbance is not sinusoidal but proceeds in a bead-string shape, as in Figure 2-9, and amplitude does not grow exponentially but is much slower.

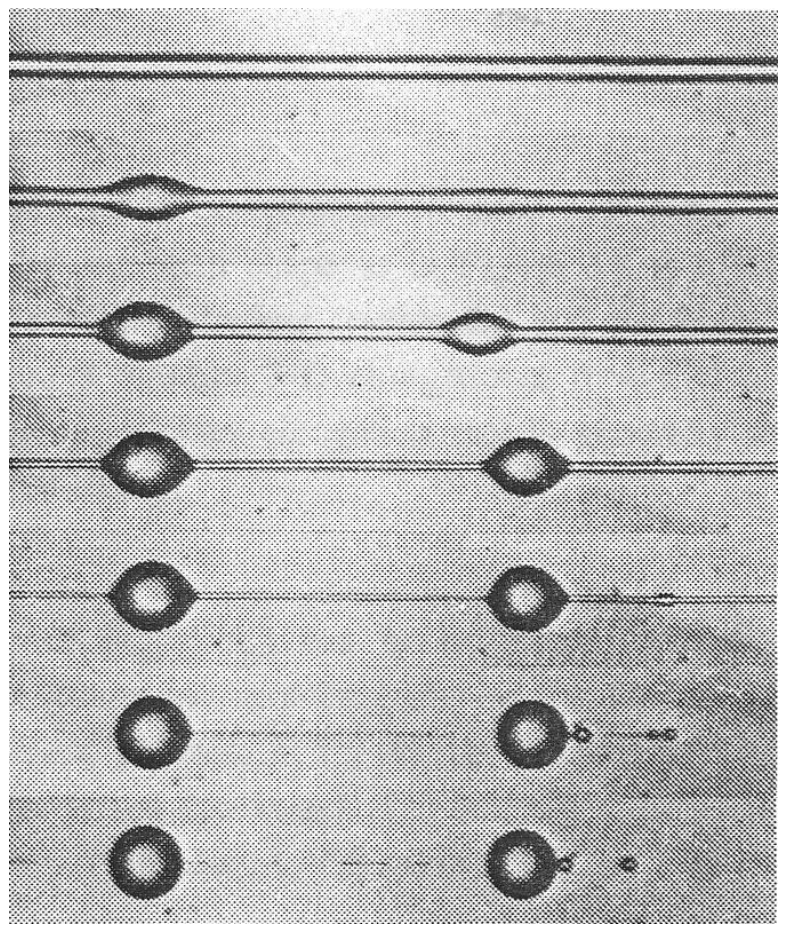

Figure 2-9: Breakup of a viscoelastic thread in a quiescent Newtonian matrix (Reproduced with permission from Janssen 1993 [11]). Thread: 80\% corn syrup/20\% water $/ 0.01 \%$ polyacrylamide, $R_{0}=0.07 \mathrm{~mm}$; matrix: silicone oil, $\eta_{\mathrm{m}}=2.0 \mathrm{~Pa} \cdot \mathrm{s} ; \sigma_{12}=18 \mathrm{mN} / \mathrm{m}$; photographs after every 3 seconds.

Viscoelastic effects due to the stresses from formation of the thread are significant if stresses build faster than they relax, i.e. the Weissenberg number $\mathrm{Wi}=\dot{\varepsilon} \lambda>1$, where $\lambda$ is the relaxation time of the thread phase, and if the total strain is substantial, i.e. $\dot{\varepsilon} t>1$. 
Furthermore, as discussed, orientational stresses may also be significant during the breakup process due to large deformation and deformation rates in the filament between droplets (beads).

Actually, in processing with extruders, bead-string breakup pattern is rarely observed for polymer blends. This may be due to elastic rupture, where the dispersed-phase elasticity is the driver for thread breakup [16]. The extensional viscosity of the thread is also a crucial parameter affecting the breakup process. However, extensional viscosity is not easily measured due to difficulties in controlling the deformation history and obtaining a steady-state [17]. For Newtonian liquids, the Trouton ratio, the ratio of extensional viscosity and simple shear viscosity, $\eta_{\mathrm{e}} / \eta_{\mathrm{s}}=3$, but for viscoelastic liquids, it can be much larger than 3 .

\subsubsection{Breakup under Flow}

Unlike breakup in a quiescent matrix where one disturbance wavelength is dominant, the disturbances on an extending thread are continuously stretched, and the disturbance wavelength that is dominant may fluctuate. Similarly, breakup occurs when a disturbance's amplitude equals the average thread radius, and the thread disintegrates into a line of droplets.

The breakup of an infinitely long liquid cylinder in general linear flows, i.e. flows that can be decomposed into 3D extension and shear components, was described by Khakhar and Ottino [18]. They assume that the shear part is negligible compared to the orientationdependent stretching rate $\dot{\varepsilon}$, but they make time dimensionless, $t^{*}$, using the orientationindependent shear rate $\dot{\gamma}$.

$$
t^{*}=\dot{\gamma} \text {. }
$$

Janssen extended the analysis incorporating the stretching efficiency $e_{f}$ :

$$
e_{\mathrm{f}}=\sqrt{2} \frac{\dot{\varepsilon}}{\dot{\gamma}} .
$$

The stretching efficiency $e_{\mathrm{f}}$ is constant for steady 2D or 3D extensional flow, $e_{\mathrm{f}}=\sqrt{1 / 2}$ or $\sqrt{2 / 3}$, respectively, but it decays toward zero for steady shear flow due to rotation of the stretching thread toward the streamline direction. At a constant stretch rate, the thread radius continuously decreases, and disturbances of all wavelengths grow but are also stretched and 
damp again to the noise level $\alpha_{0}$. After a critical time $t^{*}{ }_{\text {crit, }}$ where the thread radius is $R_{\text {crit, }}$ a fatal wavelength appears with wave number $x_{\text {crit, }}$ and grows for time $t^{*}$, until the disturbance amplitude equals the mean thread radius $R_{\text {breakup }}$ and the thread breaks up into a line of droplets. The total breakup time $t^{*}{ }_{\mathrm{b}}=t^{*}{ }_{\mathrm{crit}}+t^{*}{ }_{\mathrm{g}}$.

Janssen \& Meijer [10] condensed the analysis to 2 dimensionless groups containing the independent variables: dimensionless stretch rate $=\eta_{\mathrm{m}} \dot{\varepsilon} \alpha_{0} / \sigma$ and $p$. The dependent variables are $R_{\text {crit }} / \alpha_{0}, R_{\text {droplets }} / \alpha_{0}$, and $e_{\mathrm{f}} t_{\mathrm{g}}$. The results do not depend on $R_{0}$, as $R$ decreases affinely from $R_{0}$ to $R_{\text {crit. }}$ The results are given by Figure 2-10, Figure 2-11, and Figure 2-12. Furthermore, the total breakup time is [11]

$$
t_{\mathrm{b}}^{*}=t_{\text {crit }}^{*}+t_{\mathrm{g}}^{*}=\frac{1}{e_{\mathrm{f}}}\left[2^{3 / 2} \ln \left(\frac{R_{0}}{R_{\text {crit }}}\right)+e_{\mathrm{f}} t_{\mathrm{g}}^{*}\right] .
$$

Given the $\dot{\varepsilon}$ and $j$ and therefore $e_{\mathrm{f}}$ of a constant stretching flow and given $p, \eta_{\mathrm{m}}, \sigma_{12}$, and $\alpha_{0}$, Figure 2-10 and Figure 2-12 can be used to find $R_{\text {crit }}$ and $e_{\mathrm{ft}} t_{\mathrm{g}}$, respectively. Then, given $R_{0}$, Equation (2-28) can be used to calculate the total breakup time $t_{b}=t^{*}{ }_{b} / \gamma$ of a thread in constant

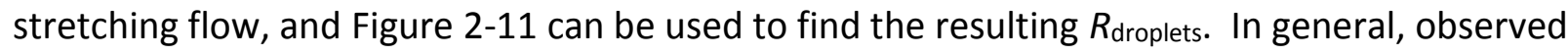
droplet sizes in practice are larger than those predicted by theory due to coalescence and early breakup caused by collisions. A collision during the breakup process (disturbance growth) can effectively create a large disturbance amplitude at the collision site and cause the thread to break up prematurely compared to breakup by disturbance growth alone. 


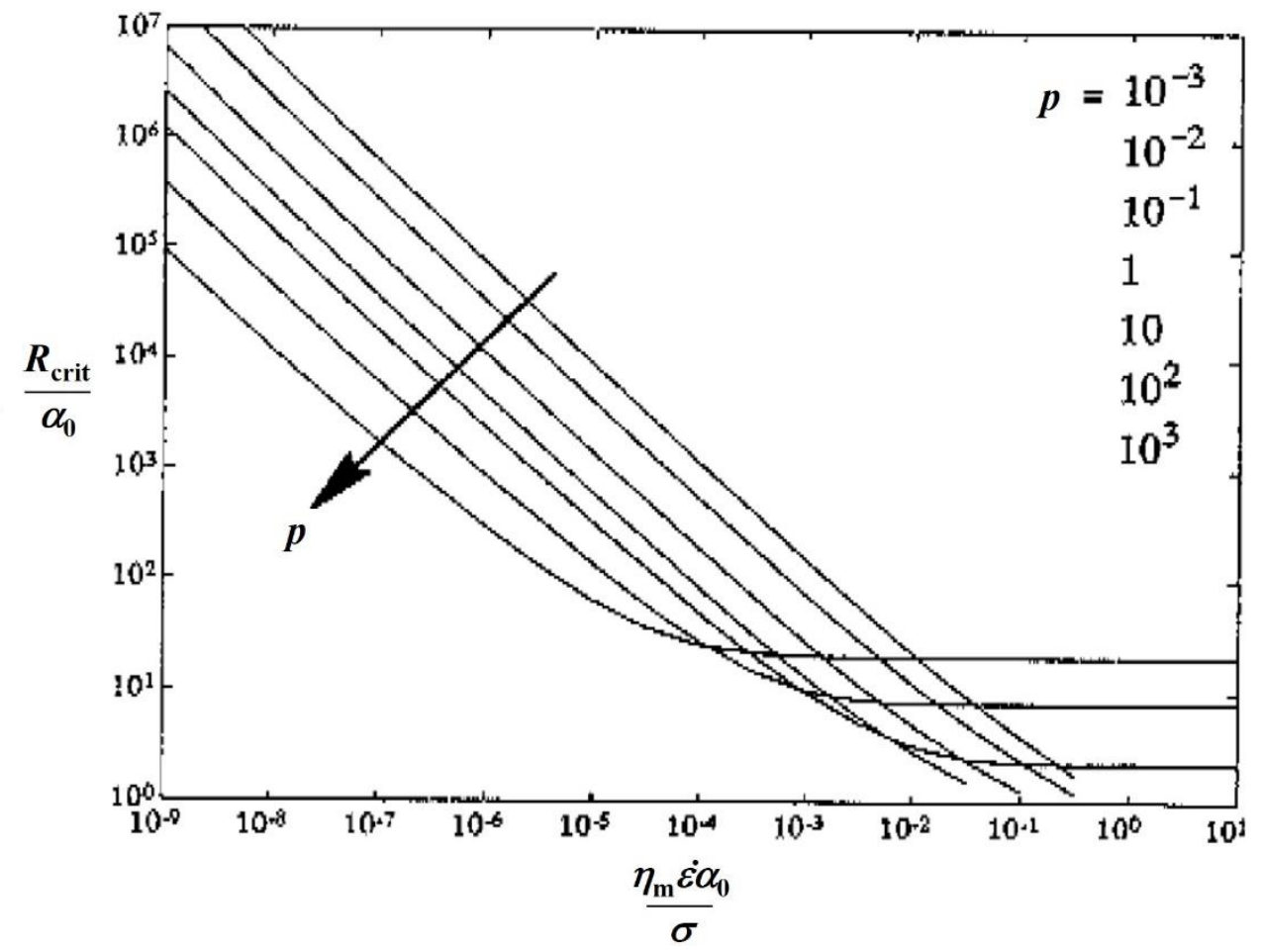

Figure 2-10: Dimensionless critical thread radius vs. dimensionless stretch rate $\& p$ (Reproduced with permission from Janssen 1993 [11]). At $R_{\text {crit, }}$ fatal disturbance starts to grow while thread is stretched at constant rate. 


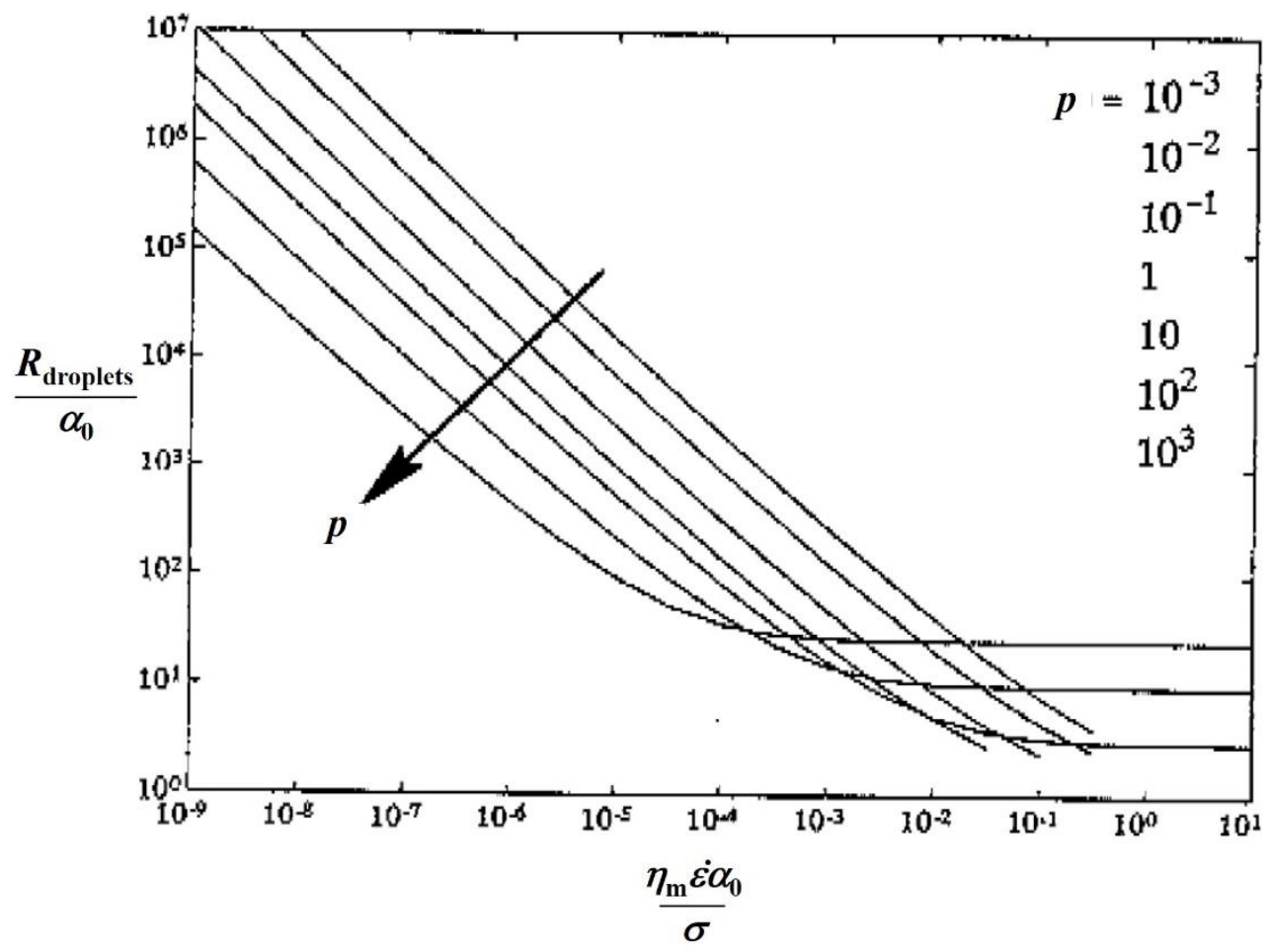

Figure 2-11: Dimensionless droplet radius vs. dimensionless stretch rate \& viscosity ratio (Reproduced with permission from Janssen 1993 [11]). Size of resulting droplets after breakup of a thread during constant stretching. 


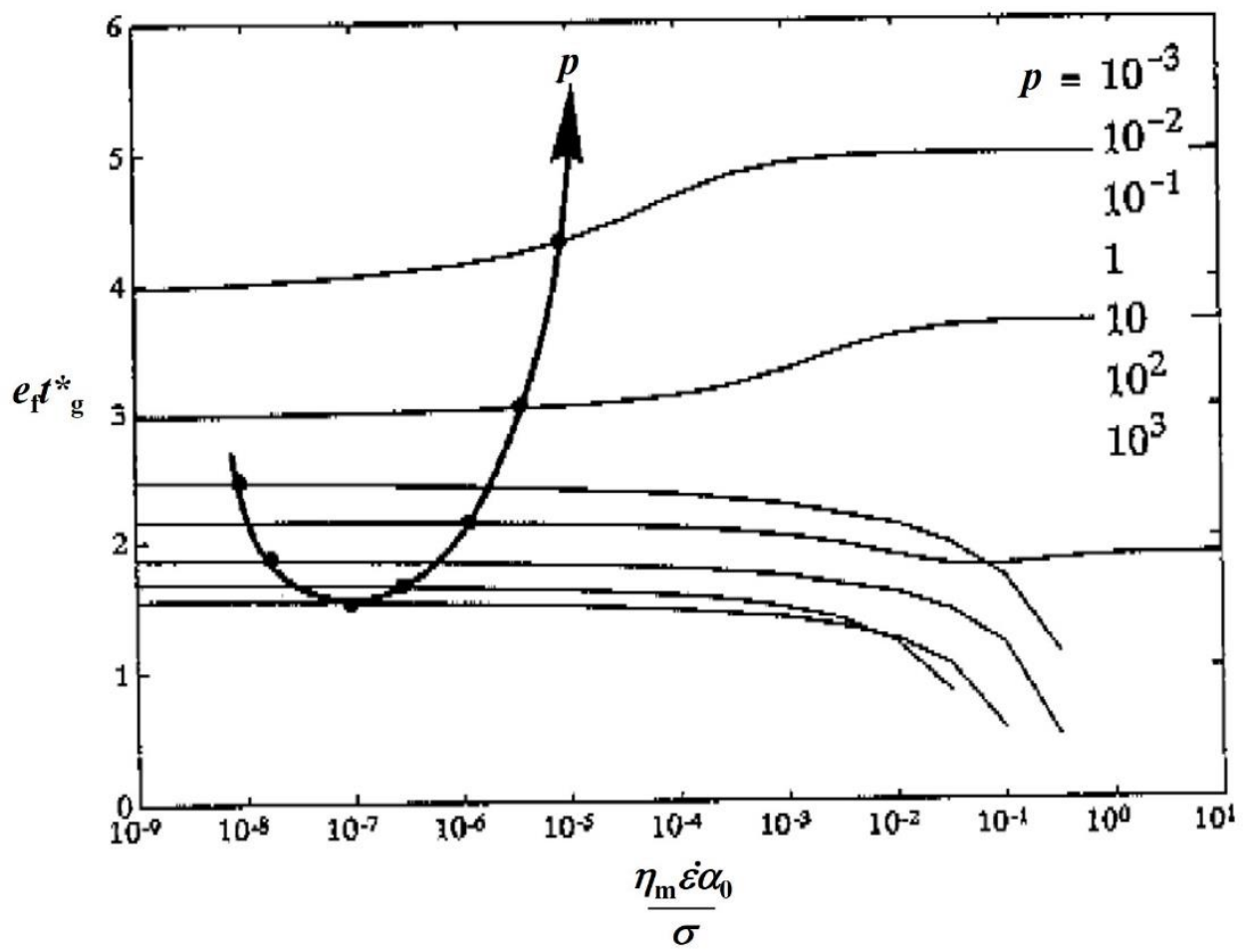

Figure 2-12: Fatal disturbance growth time vs. dimensionless stretch rate \& viscosity ratio (Reproduced with permission from Janssen 1993 [11]). Growth time after fatal disturbance, independent of type of flow, appears during constant stretching flow.

As given in Figure 2-10 and Figure 2-11, increasing the stretching rate causes the thread to thin further before breakup occurs, resulting in smaller droplets. Furthermore, in the straight line domain of Figure $2-11, R_{\text {droplets }} / \alpha_{0} \propto\left(\eta_{\mathrm{m}} \dot{\varepsilon} \alpha_{0} / \sigma_{12}\right)^{-0.9} p^{-0.45}$ or $R_{\text {droplets }} \propto\left(\eta_{\mathrm{d}} \eta_{\mathrm{m}}\right)^{-0.45}$ since stretch rate is constant. Therefore, higher viscosity of either phase or larger $p$ results in smaller droplets. At large dimensionless stretch rates, an optimum $p$ is present resulting in the smallest droplets. If $R_{0}$ » $R_{\text {crit, }}$ as in most practical situation, then $t_{\mathrm{g}}$ is negligible compared to $t_{\text {crit, }}$ and the total breakup time depends only on affine deformation from $R_{0}$ to $R_{\text {crit. }}$. Assuming the cross section of the thread remains circular, the thread radius thins as

$$
R=R_{0} \exp \left(-\frac{\dot{\varepsilon} t}{2}\right) ;
$$

therefore, the breakup time considering only affine deformation from $R_{0}$ to $R_{\text {crit }}$ is estimated by

$$
t_{\mathrm{b}} \approx t_{\text {crit }}=-\frac{2}{\dot{\varepsilon}} \ln \left(\frac{R_{\text {crit }}}{R_{0}}\right) .
$$




\subsubsection{Breakup Mechanisms}

The mechanism for drop breakup depends on $\mathrm{Ca}$ and $p$. For $\mathrm{Ca}$ » $\mathrm{Ca}_{\text {crit, }}$, see Figure 2-4, drops are stretched into long threads and capillary breakup occurs resulting in disintegration into many small droplets in one step. For Ca closer to $\mathrm{Ca}_{\text {crit, }}$ stepwise binary breakup from one drop to two daughter droplets occurs, which is the basis for the production of the Grace curve, Figure 2-4. For $\mathrm{Ca}<\mathrm{Ca}_{\text {crit, }}$ a stable drop shape is maintained, interfacial stresses outweigh viscous stresses, and breakup does not occur; though, $\mathrm{Ca}_{\text {crit }}$ depends on flow type with extensional flows leading to breakup at lower Ca than shear flows with no limit for $p$. Furthermore, drop shape depends on $p$. For $p>1$, deformed drops have rounded ends, while for $p<1$, the ends are pointed and very small droplets may break off of the ends, called tip streaming. Surfactants may facilitate this tip streaming, and they also immobilize the interface, suppressing internal flows and retarding deformation and breakup. For stepwise equilibrium breakup as in Figure 2-4, smallest droplet sizes result for $p=1$ for all flow types, but in general, transient capillary breakup during stretching results in smaller droplet sizes and finer morphologies.

Schematic representations of stepwise binary breakup and capillary breakup are shown in Figure 2-13. For stepwise binary breakup under quasi-equilibrium conditions, as in Figure 2-4, smallest droplet sizes result for $p=1$ for all flow types. However, transient capillary breakup is the dominant breakup mechanism in practice. The minimum attainable drop size in plane (2D) hyperbolic flow with stepwise binary breakup and with transient capillary breakup mechanisms are given in Figure 2-14 for various $p$. Transient capillary breakup with constant $\dot{\varepsilon}$ generally yields smaller $R_{\text {drops }}$ than stepwise binary breakup. Also, smaller drops are obtained for blends with larger $p$, as opposed to stepwise binary breakup as in Figure 2-4. The smallest drop sizes, i.e. the minimum for the $y$-axis of Figure 2-14, are limited physically to about $1 \mathrm{~nm}$ to still be considered a separate phase. Note also that if processing time permits, drops broken up by transient capillary breakup at high strain rates may still break up further by stepwise breakup mechanism. The breakup time, as in Equation (2-28) or estimated by Equation (2-30), is an important parameter for capillary breakup, and in general, stepwise binary breakup requires much longer breakup time comparatively because $\mathrm{Ca} \sim \mathrm{Ca}_{\text {crit }}$. 


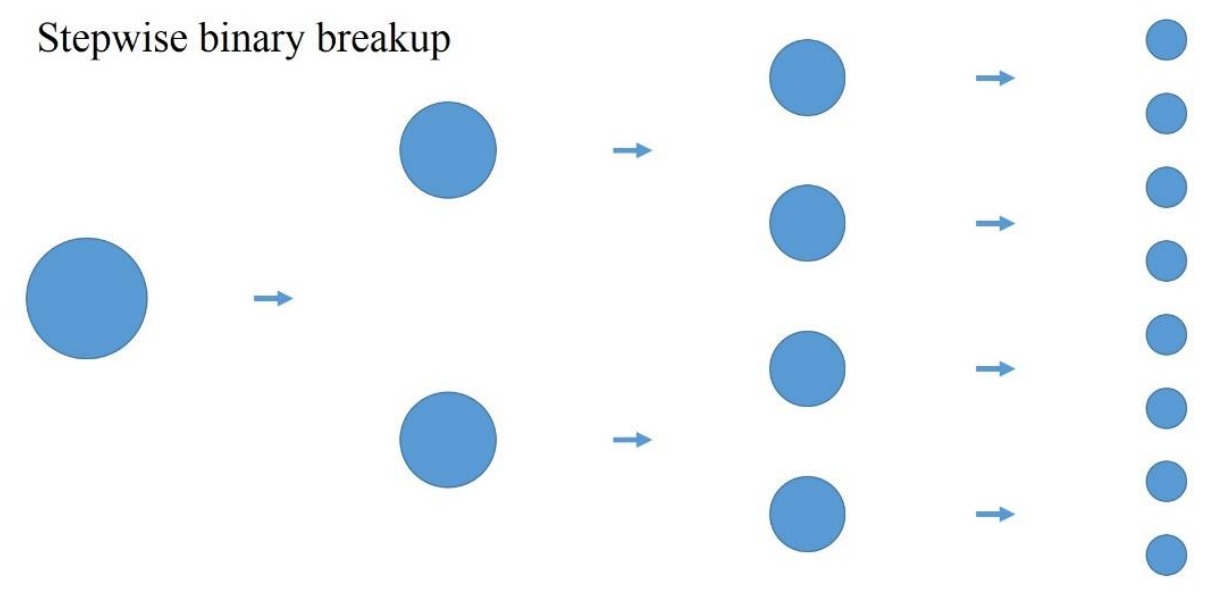

Capillary breakup

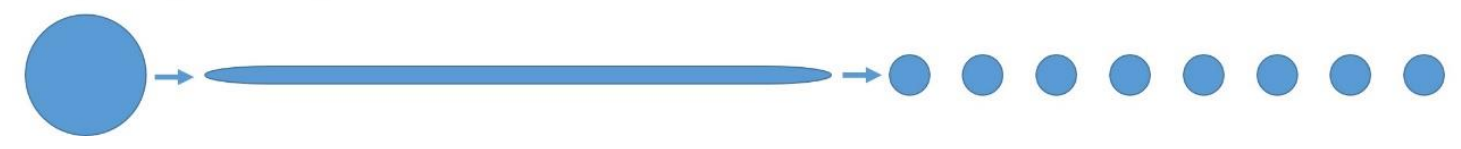

Figure 2-13: Schematic representations of stepwise binary breakup \& capillary breakup. Stepwise binary breakup at quasi-equilibrium conditions at $\mathrm{Ca} \sim \mathrm{Ca}$ crit vs. transient capillary breakup during stretching for $\mathrm{Ca} \Perp \mathrm{Ca}_{\text {crit. }}$. 


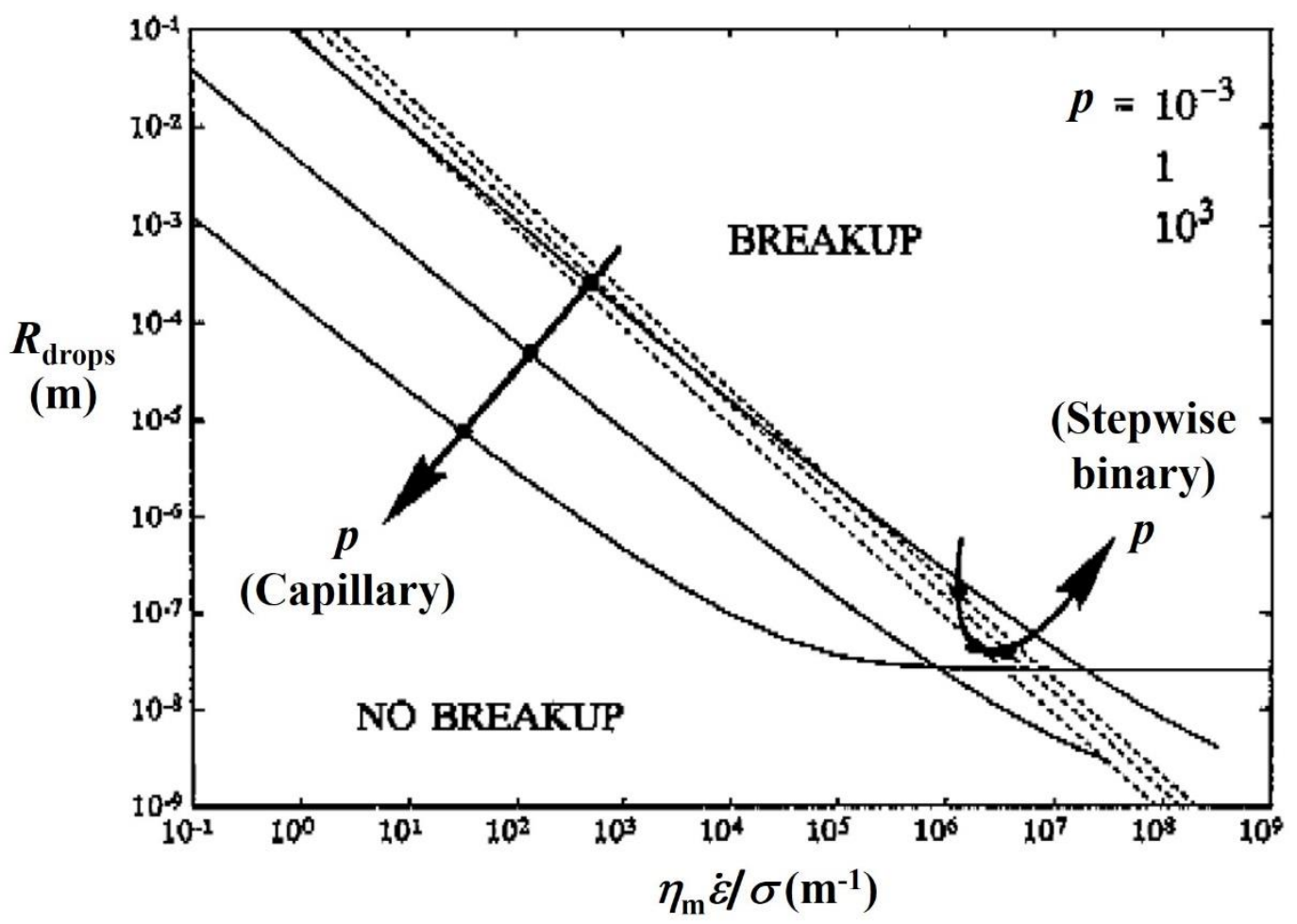

Figure 2-14: Minimum drop size in plane hyperbolic extensional flow (Reproduced with permission from Janssen 1993 [11]). Dashed lines - Stepwise, binary, equilibrium breakup mechansim at $\mathrm{Ca} \sim \mathrm{Ca}_{\text {crit; }}$ Solid lines - Transient, capillary breakup mechanism at $\mathrm{Ca}$ » $\mathrm{Ca}_{\text {crit. }}$.

Two competitive processes occur on an extended drop in a quiescent matrix: $a$ ) retraction toward a sphere due to the pressure difference caused by difference in interface shape between the ends and the drop center and $b$ ) growth of capillary waves leading to breakup. Whether the drop totally retracts or breaks up depends on the initial extension of the drop and viscosity ratio and and flow type [19]. In general, larger $p$ retards the growth of instabilities leading to capillary breakup and requires larger extension for breakup to occur rather than drop relaxation. Drop relaxation is a common method for measuring interfacial tension.

Viscoelasticity in either of the two phases generally retards deformation and breakup; though, it is only significant if the deformation is fast and large enough, i.e. $\dot{\varepsilon} \lambda>1$ and $\dot{\varepsilon} t>1$. Hence, it does not significantly affect quasi-equilibrium drop deformation experiments since deformation rates are small and stresses quickly relax. For drop relaxation, though, viscoelastic 
drops require larger extensions for breakup mechanisms to outweigh drop retraction than do Newtonian drops since growth of capillary waves is largely retarded.

\subsubsection{Coalescence}

The final morphology of an immiscible blend depends on the dynamics of and the interaction between drop breakup and coalescence. Drop deformation and breakup tend to decrease the size of the dispersed phase, while coalescence tends to increase it. Coalescence occurs during the collision of two droplets. The interface of colliding droplets flattens and forms a thin film of the matrix fluid between the droplets. The thin film must drain until a critical film thickness $h_{\text {crit, }}$ typically about $10 \mathrm{~nm}$ [20], is reached, at which point van der Waals forces cause the attraction which ruptures the film and the droplets coalesce. Film drainage requires time and is considered the rate-limiting step; if the time for film drainage is greater than the interaction time between drops during a collision, due to hydrodynamic forces, then the droplets will bounce off of one another and not coalesce.

Models for coalescence combine the frequency of collisions and the probability that a collision will result in coalescence. The frequency of collisions is dependent on the volume fraction of the dispersed phase, flow conditions, and processing time. For equally sized spherical drops in simple shear flow, the collision frequency $C$ of a drop is

$$
C=\frac{8}{\pi} \dot{\gamma} \phi
$$

which interestingly does not depend on drop radius [11]. Using Equation (2-31), the average time between collisions for each drop is $t_{\text {coll }}=1 / C$. The probability that a collision will occur during processing, $P_{\text {coll, }}$ then depends on the characteristic processing time $t_{\text {proc: }}$ :

$$
P_{\text {coll }}=\exp \left(-\frac{t_{\text {coll }}}{t_{\text {proc }}}\right)=\exp \left(\frac{-\pi}{8 \dot{\gamma} \phi t_{\text {proc }}}\right),
$$

which varies from zero for $t_{\text {proc }}$ " $t_{\text {coll }}$ to one for $t_{\text {proc }} " t_{\text {coll. }}$.

The assumptions used to develop film drainage models depend on the viscosity ratio $p$. For $p$ « 1, the droplet fluid provides no resistance to drainage, and the model assumes a fully mobile interface (FMI). For $p \approx 1$, viscous stresses within the droplet are the main resistance to 
film drainage, and the model assumes a partially mobile interface (PMI). Finally, for $p » 1$, the interface does not deform in response to shear stresses in the film during drainage: an immobile interface (IMI). Schematics for these three systems are shown in Figure 2-15 for equally sized drops colliding symmetrically with flattened, parallel interfaces between them and with a constant contact force $F$, all of which are also assumptions made for the simplest film drainage models.
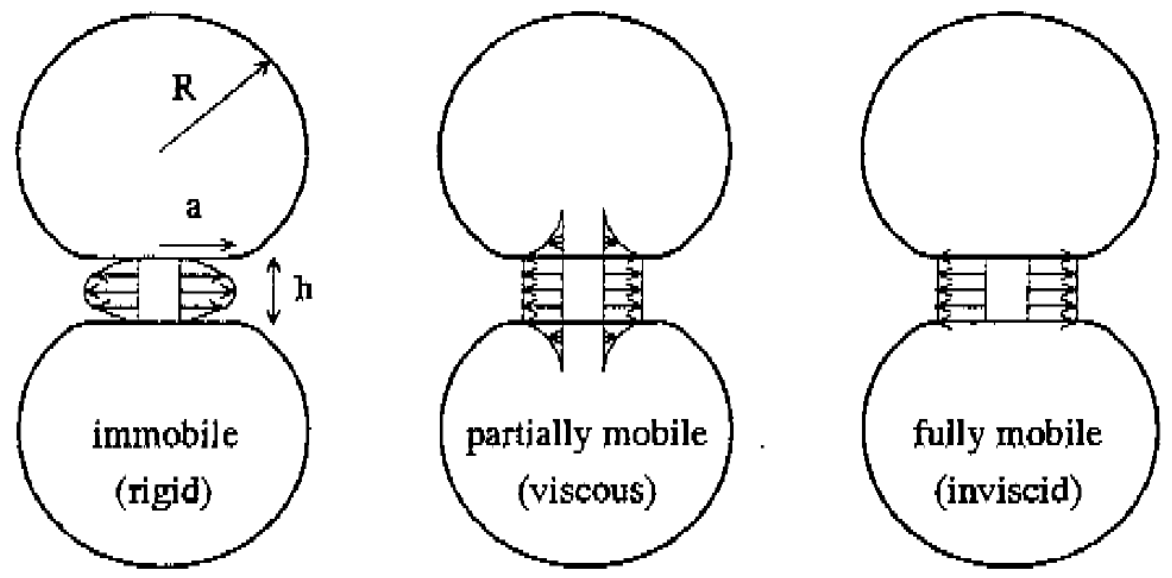

Figure 2-15: Interface mobility during film drainage (Reproduced with permission from Janssen 1993 [11]). Schematics of velocity distribution during film drainage for immobile, partially mobile, and fully mobile interfaces.

Using the previous assumptions and the boundary conditions shown in Figure 2-15, models have been developed to give the rate of film drainage, $-d h / d t$. Then, to calculate the required drainage time, $t_{\text {drain, }}$ these relations may be integrated from $h_{0}$, the initial film thickness at which drop interaction begins, to $h_{\text {crit, }}$ the critical thickness at which van der Waals forces become important and the interface between the drops ruptures. The constant contact force $F$ may be estimated from the Stokes drag force acting on the drops, which for simple shear flow is [11] (note should also include a term for effective viscosity of the dispersion, as in Ch. 5 of Janssen diss.)

$$
F=6 \pi \eta_{\mathrm{m}} \dot{\gamma} R^{2}
$$


Additionally, the initial film thickness $h_{0}$ is the thickness at which the film drainage model's $d h / d t$ equals the constant approach velocity at large distances due to flow $j R$, and the critical film thickness $h_{\text {crit }}$ is estimated using [11]

$$
h_{\text {crit }}=\left(\frac{A R}{8 \pi \sigma_{12}}\right)^{1 / 3},
$$

where $A$ is the material-dependent Hamaker constant (around $10^{-20} \mathrm{~J}$ ). For most blends, $h_{\text {crit }}$ is approximately $10 \mathrm{~nm}$ [20]. Table 2-1 summarizes the important relations for these film drainage models. Note that the capillary number $C a$ is $\eta_{\mathrm{m}} \dot{\gamma} R / \sigma_{12}$, and the viscosity ratio $p$ is $\eta_{\mathrm{d}} / \eta_{\mathrm{m}}$

Table 2-1: Film drainage models for different interface mobility assumptions [11].

\begin{tabular}{|c|c|c|c|}
\hline Interface mobility & $\begin{array}{l}\text { Rate of film } \\
\text { drainage, }-\mathrm{dh} / \mathrm{dt}\end{array}$ & Drainage time, $\mathrm{t}_{\text {drain }}$ & $\begin{array}{l}\text { Initial film thickness, } \\
\mathrm{h}_{0}\end{array}$ \\
\hline $\begin{array}{c}\text { Immobile } \\
\text { interface }\end{array}$ & $\frac{8 \pi \sigma_{12}^{2} h^{3}}{3 \eta_{\mathrm{m}} R^{2} F}$ & $\frac{3 \eta_{\mathrm{m}} R^{2} F}{16 \pi \sigma_{12}^{2}}\left(\frac{1}{h_{\text {crit }}^{2}}-\frac{1}{h_{0}^{2}}\right)$ & $R\left(\frac{3}{2} C a\right)^{2 / 3}$ \\
\hline $\begin{array}{c}\text { Partially mobile } \\
\text { interface }\end{array}$ & $\frac{2\left(2 \pi \sigma_{12} / R\right)^{3 / 2} h^{2}}{\pi \eta_{\mathrm{d}} F^{1 / 2}}$ & $\frac{\pi \eta_{\mathrm{d}} F^{1 / 2}}{2\left(2 \pi \sigma_{12} / R\right)^{3 / 2}}\left(\frac{1}{h_{\text {crit }}}-\frac{1}{h_{0}}\right)$ & $R\left(\frac{3}{16}\right)^{1 / 4} p^{1 / 2} C a^{3 / 4}$ \\
\hline $\begin{array}{c}\text { Fully mobile } \\
\text { interface }\end{array}$ & $\frac{2 \sigma_{12} h}{3 \eta_{\mathrm{m}} R}$ & $\frac{3 \eta_{\mathrm{m}} R}{2 \sigma_{12}} \ln \left(\frac{h_{0}}{h_{\text {crit }}}\right)$ & $R \frac{3}{2} C a$ \\
\hline
\end{tabular}

As expected, for all interface mobility assumptions, larger drops (larger $R$ ) require longer drainage times because the flattened area, given by $\pi a^{2}$ as in Figure 2-15, is larger, therefore, requiring more liquid to drain. For unequally sized drops, an equivalent drop radius is sometimes used [11]

$$
R_{\text {eq }}^{-1}=\frac{R_{1}^{-1}+R_{2}^{-1}}{2},
$$

which varies from $R_{1}$ to $2 R_{1} ; R_{v}$ is also often used. Also, interestingly, larger $F$ yields longer drainage times; this is again explained by larger flattened area. Using a simple pressure balance, equating contact force/contact area to interfacial tension, yields 


$$
a=\sqrt{\frac{F R}{2 \pi \sigma_{12}}},
$$

and substituting $F$ as in Equation (2-33) yields

$$
a=R \sqrt{3 C a} .
$$

Therefore, lower $F$ or lower $C a$ decreases $a$ and decreases the amount of liquid for film drainage, increasing the likelihood of coalescence. To determine the appropriate interface mobility assumption, Table 2-2 may be used; if all the conditions listed are satisfied, then the model assuming partially mobile interfaces may be used. Furthermore, an additional condition should be satisfied which restricts $a$ with respect to $R$ [11]:

$$
\frac{a}{R}<1 \text { or } R<\frac{1}{3}\left(\frac{\eta_{\mathrm{m}} \dot{\gamma}}{\sigma_{12}}\right)^{-1} .
$$

For Table $2-2, h_{\text {crit }}$ should be used for $h$, since the last stage of film drainage is rate-limiting. 
Table 2-2: Conditions to deviate from partially mobile interface model [11].

\begin{tabular}{|l|l|}
\hline Condition & Action if condition not met \\
\hline$p>\frac{6 h}{a}$ or $R>\left(\frac{12 h^{2}}{p^{2}}\right)^{1 / 3}\left(\frac{\eta_{\mathrm{m}} \dot{\gamma}}{\sigma_{12}}\right)^{-1 / 3}$ & Transition to fully mobile interfaces \\
\hline$\frac{a}{R}>2\left(\frac{h}{R}\right)^{1 / 2}$ or $R>\left(\frac{p^{2} h^{2}}{27}\right)^{1 / 3}\left(\frac{\eta_{\mathrm{m}} \dot{\gamma}}{\sigma_{12}}\right)^{-1 / 3}\left(\frac{\eta_{\mathrm{m}} \dot{\gamma}}{\sigma_{12}}\right)^{-1 / 2}$ & Transition to immobile interfaces \\
\hline$h<\frac{\eta_{\mathrm{d}}^{2} R}{4 \rho_{\mathrm{d}} \sigma_{12} a}$ or $R<\frac{\eta_{\mathrm{d}}^{4}}{48 h^{2} \rho_{\mathrm{d}}^{2} \sigma_{12}^{2}}\left(\frac{\eta_{\mathrm{m}} \dot{\gamma}}{\sigma_{12}}\right)^{-1}$, & Transition to drainage without flattening \\
where $\rho_{\mathrm{d}}$ is dispersed-phase density & stationary boundary layer inside the drops) \\
\hline
\end{tabular}

Similar to the collision probability in Equation (2-32), the probability that film drainage occurs completely, $P_{\text {drain, }}$ may be calculated as

$$
P_{\text {drain }}=\exp \left(-\frac{t_{\text {drain }}}{t_{\text {int }}}\right),
$$

where $t_{\text {int }}$ is the interaction time for drops during collision, given by

$$
t_{\text {int }}=\frac{1}{\dot{\gamma}}
$$

for drops in simple shear flow. Assuming that $h_{0} » h_{\text {crit, }} 1 / h_{0}^{2}$ and $1 / h_{0}$ are negligible in the $t_{\text {drain }}$ equations in Table 2-1 for immobile and partially mobile interfaces, respectively, and $h_{0}$ is approximated by $R$ for $t_{\text {drain }}$ for fully mobile interfaces. The resulting drainage probabilities for the different interface mobility models are given in Table 2-3. 
Table 2-3: Drainage probability for various interfacial mobilities [11].

\begin{tabular}{|l|r|}
\hline Interface mobility & Drainage probability, $\mathrm{P}_{\text {drain }}$ \\
\hline Interface & $\exp \left[-\frac{9}{8}\left(\frac{R}{h_{\text {crit }}}\right)^{2} \mathrm{Ca}^{2}\right]$ \\
\hline $\begin{array}{l}\text { Partially mobile } \\
\text { interface }\end{array}$ & $\exp \left(-\frac{\sqrt{3}}{4} \frac{R}{h_{\text {crit }}} \mathrm{pCa}^{3 / 2}\right)$ \\
\hline Fully mobile & $\exp \left[-\frac{3}{2} \mathrm{Ca} \ln \left(\frac{R}{h_{\text {crit }}}\right)\right]$ \\
\hline
\end{tabular}

Coalescence occurs when both drop collision and film drainage take place; therefore, the overall coalescence probability, $P_{\text {coa, }}$ is

$$
P_{\text {coa }}=P_{\text {coll }} \cdot P_{\text {drain }}=\exp \left(-\frac{t_{\text {coll }}}{t_{\text {proc }}}-\frac{t_{\text {drain }}}{t_{\text {int }}}\right) .
$$

Figure 2-16 shows the dependence of collision, drainage, and overall coalescence probabilities on drop size and the flow parameter for a specific situation. As given by Equation (2-32), collisions occur when flow is fast enough, and $P_{\text {coll }}$ does not depend on $R$. Conversely, film drainage is more likely for slower flow, depending on $R$, with drainage being easier for smaller drops. The transition from 1 to 0 for $P_{\text {drain }}$ occurs at higher flow for smaller drops. Combining these, $P_{\text {coa }}$ appears as a hump, where coalescence occurs when flow is fast enough for collisions to take place but not too fast so that film drainage is complete for a given drop size. 

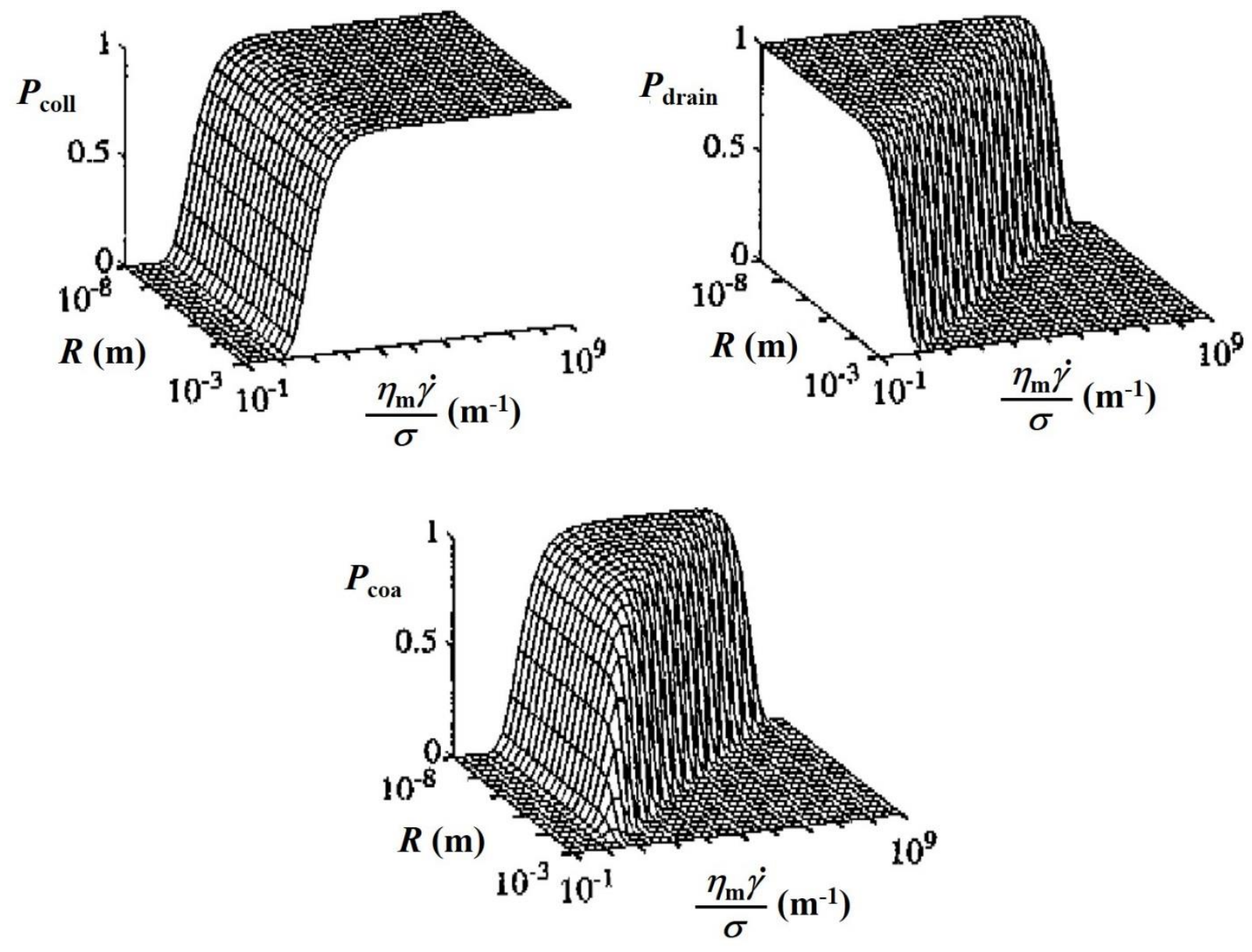

Figure 2-16: Probabilities associated with coalescence vs. radius \& flow parameter (Reproduced with permission from Janssen 1993 [11]). $P_{\text {coll }} \equiv$ collision probability; $P_{\text {drain }} \equiv$ drainage probability; and $P_{\text {coa }} \equiv$ coalescence probability. Plots are for simple shear flow, $t_{\text {proc }}=$ $50 \mathrm{~s}, \eta_{\mathrm{d}}=\eta_{\mathrm{m}}=1 \mathrm{~Pa} \cdot \mathrm{s}, \sigma=10 \mathrm{mN} / \mathrm{m}, h_{\text {crit }}=5 \mathrm{~nm}, \phi=0.1$, partially mobile interfaces.

Figure 2-17 shows the dependence of coalescence probability on the capillary number; the plot is constructed by multiplying cross sections of $P_{\text {coa }}$ vs. $\eta_{\mathrm{m}} \dot{\gamma} / \sigma_{12}$ for various $R$ values by $R$, yielding the capillary number Ca. The window for coalescence is much wider for smaller $R$ as expected. Also shown in Figure 2-17 is $\mathrm{Ca}_{\text {crit }}$ for the situation of Figure 2-16. It is apparent then that coalescence occurs at much smaller Ca than breakup. Therefore, breakup takes place in regions of strong flow, and coalescence takes place in regions of weak flow. 


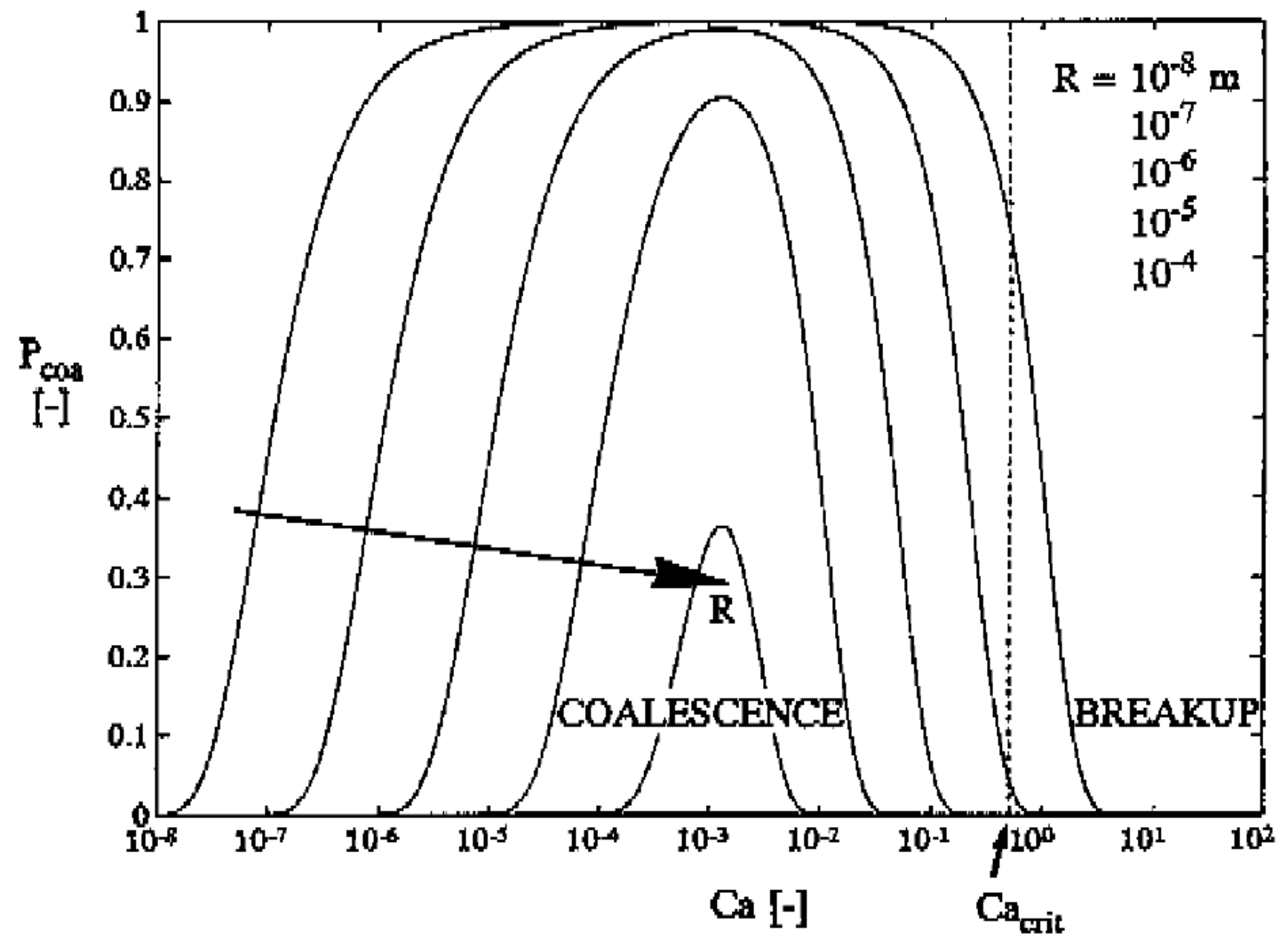

Figure 2-17: Coalescence probability vs. capillary number for various drop radii (Reproduced with permission from Janssen 1993 [11]). Same situation as in Figure 2-16.

Given the steep slopes in the regions where coalescence probability changes from zero to one in Figure 2-16, $P_{\text {coa }}$ can be reasonably estimated by a binary function which equals either zero or one depending on certain criteria. Generally, film drainage is the rate-limiting step of coalescence, and depending on concentration of the dispersed polymer phase, $P_{\text {coll }}$ is usually high. Therefore, it is reasonable to assume $P_{\text {coll }}=1$, and $P_{\text {coa }}=P_{\text {drain }}$ which will equal 1 if $t_{\text {int }} \geq$ $t_{\text {drain }}$ and 0 if $t_{\text {int }}<t_{\text {drain. }} . P_{\text {coa }}$ transitions from 0 to 1 then when $t_{\text {int }}=t_{\text {drain, }}$ from which a critical drop size $R_{\max }$ may be calculated below which coalescence occurs and above which it does not. The maximum drop size for coalescence is given in Table 2-4 for each interface mobility model. 
Table 2-4: Maximum drop size for coalescence [11].

\begin{tabular}{|l|l|}
\hline Interface mobility & Maximum drop radius, $\mathrm{R}_{\max }$ \\
\hline Immobile & $R_{\max }=\left(\frac{8}{9}\right)^{1 / 4} h_{\text {crit }}^{1 / 2}\left(\frac{\eta_{\mathrm{m}} \dot{\gamma}}{\sigma_{12}}\right)^{-1 / 2}$ \\
\hline $\begin{array}{l}\text { Partially mobile } \\
\text { interface }\end{array}$ & $R_{\max }=\left(\frac{4}{\sqrt{3}} \frac{h_{\text {crit }}}{p}\right)^{2 / 5}\left(\frac{\eta_{\mathrm{m}} \dot{\gamma}}{\sigma_{12}}\right)^{-3 / 5}$ \\
\hline $\begin{array}{l}\text { Fully mobile } \\
\text { interface }\end{array}$ & $R_{\max } \ln \left(\frac{R_{\max }}{h_{\text {crit }}}\right)=\frac{2}{3}\left(\frac{\eta_{\mathrm{m}}}{\sigma_{12}}\right)^{-1}$ \\
\hline
\end{tabular}

\subsubsection{Overall Morphology Development for Viscoelastic Blends}

Janssen offered a useful analysis of the kinetics of morphology development in the extrudate strands of polymer blended material after exiting the die head of an extruder and before entering a cooling water bath [11]. Depending on the time between the strand exiting the die and entering the water bath, the morphology may be thread-in-matrix or drop-inmatrix. Flow through the die is highly extensional and is a strong mixing zone, while flow in the strand before quenching in water is a quiescent, weak zone. The total extensional strain through the convergence of the die, $\varepsilon$, is given by

$$
\varepsilon=\ln \left(\frac{\text { Area }_{1}}{\text { Area }_{2}}\right)=\ln \left[\left(\frac{d_{1}}{d_{2}}\right)^{2}\right],
$$

where $d_{i}$ is the diameter of the cross section at the die entrance $(i=1)$ or exit $(i=2)$. The average extensional strain rate $\overline{\dot{\varepsilon}}$ through the die may then be calculated given the residence time in the die $t_{\text {res: }}$

$$
\overline{\dot{\varepsilon}}=\frac{\varepsilon}{t_{\text {res }}} \text {. }
$$

Then, given the initial drop size, viscosity of each phase, and interfacial tension, the capillary number may be calculated according to Equation (2-8). If $\mathrm{Ca} / \mathrm{Ca}$ crit is greater than 
about 5, the drop will stretch affinely according to Equation (2-16), where the width of the extending thread $(B=2 R)$ decreases exponentially according to

$$
\frac{B}{2 R_{0}}=\frac{R}{R_{0}}=e^{-\varepsilon} .
$$

If $R \leq R_{\text {crit }}$ as given in Figure 2-10, then the extending thread will break up into droplets sized according to Figure 2-11. Otherwise, the extended thread will be stable after passing through the die. As the polymer strand is transported through air after exiting the die, the amount of time for capillary breakup in quiescent flow may be calculated according to Equation (2-25). If the time for the strand to go from the die exit to the water bath is longer than $t_{b}$, then the extended thread of the dispersed phase will break up into droplets sized according to Equation (2-22) with $x_{m}$ given in Figure 2-7. Otherwise the thread-in-matrix morphology is quenched in place in the final material. Furthermore, the time before quenching should also be minimized to avoid coalescence in the quiescent strand.

The focus of this work is dispersive mixing as quantified by the size and size distribution of the dispersed-phase domains. Distributive mixing is also important for good overall mixing and is quantified by the minimum spacing and distribution of spacing between dispersed-phase domains. Re-orientation of the dispersed phase is efficient to impart distributive mixing and effectively mix dispersed solid agglomerates and aggregates as well as liquid dispersed phases into polymer matrices. However, distributive mixing was not studied in detail in this work.

\subsection{Nanoparticles in Blends}

The addition of nanoparticles into colloidal systems was first described by Pickering and Ramsden [21]. S.U. Pickering described in 1907 the stabilization of paraffin oil-in-water emulsions by various types of solid particles adsorbed at the oil droplet surface and showed that this strategy improved the stability compared to surfactant-based emulsions. W. Ramsden described in 1903 the adsorption of solid particles at air-water interfaces and studied their influences over foaming and the recovery of solid particles. These systems of solid particles adsorbed at the interface between phases in binary colloidal blends are called Pickering- 
Ramsden systems. This stabilization strategy may be extended to high-molecular weight blends of immiscible polymers. The background and theory associated with this compatibilization strategy are described in this section.

\subsubsection{Thermodynamic Effects}

The equilibrium localization of a nanoparticle in a mixture of immiscible liquids is given by the interfacial thermodynamics of the system. The nanoparticles may be localized in one liquid phase or the other, at the interface between the two liquids, or a combination of the above. Kinetic effects are very influential in molten polymers, however, due to their high viscosities, the complex flow fields that are created during processing, and other rheological characteristics; therefore, equilibrium localization is not always reached. The surface Gibbs free energy, $G$, of a single, isolated nanoparticle at the liquid-liquid interface, either temporarily or at equilibrium, is given by [22]

$$
G=A_{\mathrm{s}-1} \gamma_{\mathrm{s}-1}+A_{\mathrm{s}-2} \gamma_{\mathrm{s}-2}-A_{12} \gamma_{12},
$$

where $A_{s-i}$ and $\gamma_{s-i}$ are the interfacial area and interfacial tension between the solid nanoparticle and each liquid, respectively, $A_{12}$ is the displaced interfacial area between the liquids, and $\gamma_{12}=$ $\sigma_{12}$, the interfacial tension between the liquids. These are shown schematically in Figure 2-18. The interfacial areas between the nanoparticle and each liquid are spherical surfaces and total to $4 \pi R^{2}$, and the displaced interfacial area between the liquids is a planar surface. 


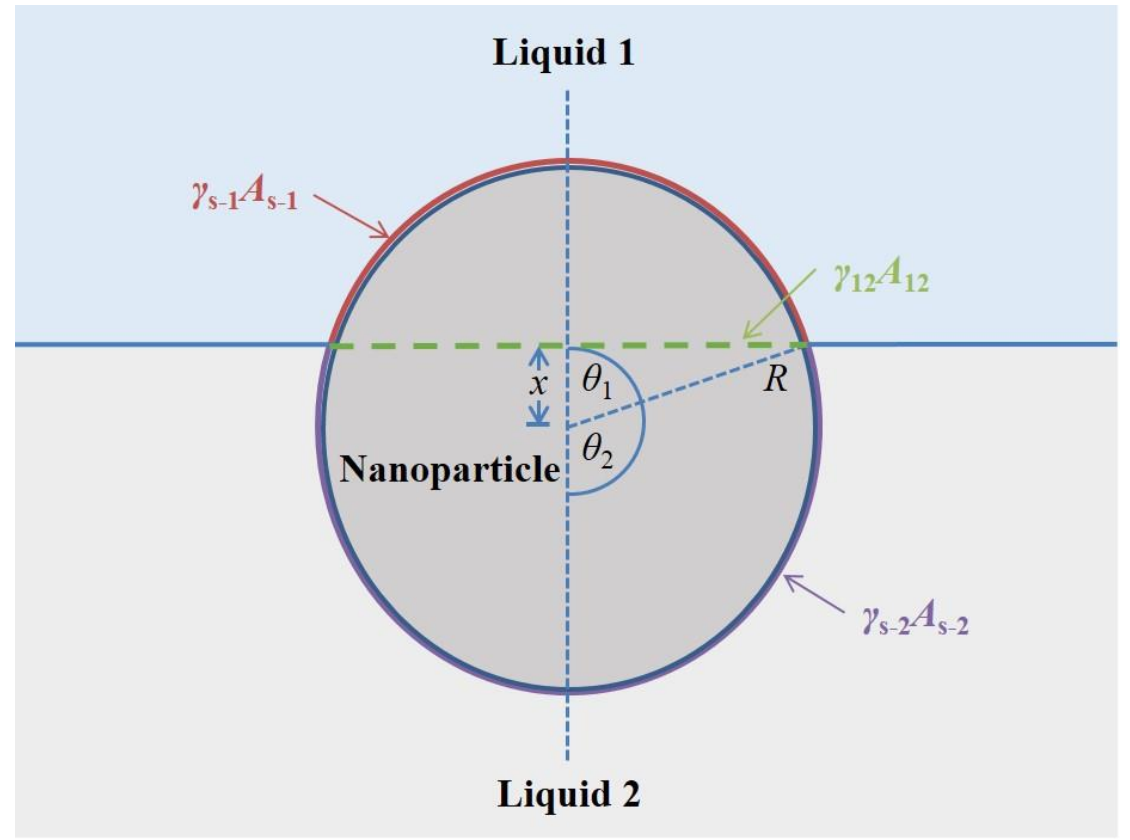

Figure 2-18: Schematic of nanoparticle at liquid-liquid interface. Spherical nanoparticle of radius $R$ located with center at distance $x$ from interface between two immiscible liquids with interfacial tensions and areas between nanoparticle and respective liquids labeled as well as interfacial tension and displaced area between the liquids. Contact angles through each liquid are also defined.

Assuming that $-R \leq x \leq R$, i.e. the nanoparticle is localized the interface, geometrical relations reduce Equation (2-45) to [22]

$$
G=2 \pi R^{2}\left\{\left(1-\frac{x}{R}\right) \gamma_{\mathrm{s}-1}+\left(1+\frac{x}{R}\right) \gamma_{\mathrm{s}-2}-\frac{1}{2}\left[1-\left(\frac{x}{R}\right)^{2}\right] \gamma_{12}\right\},
$$

which reduces to $G=4 \pi R^{2} \gamma_{s-1}$ when $x=-R$ and is defined as such for $x<-R$ (complete wetting of the nanoparticle by Liquid 1) and to $G=4 \pi R^{2} \gamma_{\mathrm{s}-2}$ when $x=R$ and is defined as such for $x>R$. Equation (2-46) is plotted in Figure 2-19 for the case when $\gamma_{s-1}>\gamma_{s-2}$ with various relations for $\gamma_{12}$. As shown, the lowest energy position is in Liquid $2(x \geq R)$ when

$$
\gamma_{12} \leq \gamma_{\mathrm{s}-1}-\gamma_{\mathrm{s}-2}
$$

An equivalent relation is the spreading coefficient, $S_{2-1}$

$$
S_{2-1}=\gamma_{\mathrm{s}-1}-\gamma_{\mathrm{s}-2}-\gamma_{12}
$$

which is the same as Relation (2-47) if

$$
S_{2-1} \geq 0
$$


and indicates that Liquid 2 will displace Liquid 1 on the surface of the solid nanoparticle.

Therefore, if Relations (2-47) and (2-49) hold, then a nanoparticle crossing the interface from Liquid 1 toward Liquid 2 will experience a positive driving force at all points for $-R \leq x \leq R$.

The force acting on the nanoparticle as a result of interfacial tensions is [22]

$$
-\frac{\partial G}{\partial x}=2 \pi R\left[\gamma_{\mathrm{s}-1}-\gamma_{\mathrm{s}-2}-\left(\frac{x}{R}\right) \gamma_{12}\right]
$$

which is positive at all $x$ between $-R$ and $R$ when

$$
\gamma_{12} \leq \gamma_{\mathrm{s}-1}-\gamma_{\mathrm{s}-2}
$$

Therefore, in this case, a nanoparticle crossing the interface due to kinetic motion experiences an interfacial driving force.

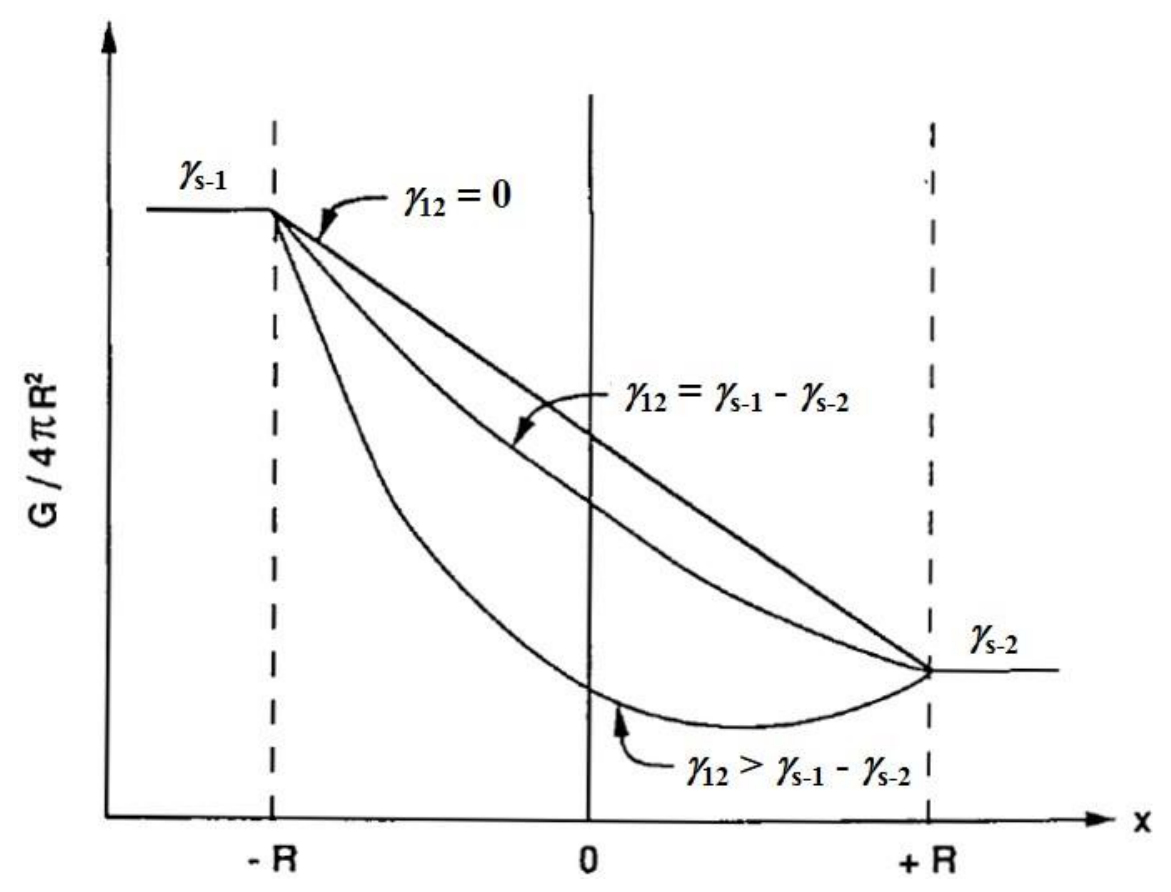

Figure 2-19: Surface Gibbs free energy for a nanoparticle at interface (Reproduced from Cheng, Keskkula, and Paul, Polymer, 33, 8, 1606-1619, 1992 [22], Copyright 1992, with permission from Elsevier). Surface Gibbs free energy curves with respect to $x$ for a particle of radius $R$ localized at the liquid-liquid interface for $\gamma_{s-1}$.

Partial wetting requires that the energy of adhesion between phases, $E_{\mathrm{adh}, 1-2}$, be positive and the spreading coefficient, $S_{1-2}$, be negative [21]: 


$$
\begin{gathered}
E_{\mathrm{adh}, 1-2}=\gamma_{\mathrm{s}-2}+\gamma_{12}-\gamma_{\mathrm{s}-1}>0 \\
\text { and } S_{1-2}=\gamma_{\mathrm{s}-2}-\gamma_{12}-\gamma_{\mathrm{s}-1}<0,
\end{gathered}
$$

which is equivalent to the stipulation given elsewhere that the wettability parameter, $\omega_{1-2}$, [23]

$$
\omega_{1-2}=\cos \theta_{1}=\frac{\gamma_{\mathrm{s}-2}-\gamma_{\mathrm{s}-1}}{\gamma_{12}}
$$

be between -1 and 1 , corresponding to a definable contact angle:

$$
\left|\omega_{1-2}\right|<1 ; 0<\theta_{1}<180^{\circ} .
$$

The contact angle through the other phase is defined as

$$
\cos \theta_{2}=\frac{\gamma_{\mathrm{s}-1}-\gamma_{\mathrm{s}-2}}{\gamma_{12}}=\omega_{2-1}=\pi-\theta_{1} .
$$

Therefore, for partial wetting with definable contact angles through each phase, particles cannot be too hydrophilic, which would result in complete wetting by the more polar phase ( $S_{1-2}$ or $S_{2-1} \geq 0$ ), or too hydrophobic, which would correspondingly result in localization in the more nonpolar phase. In fact, adsorption has often been reported to be strongest, or the resulting emulsion to be most stable, when the contact angle is $90^{\circ}$ [21], and indeed the free energy of adsorption (actually defined as the free energy input required to desorb the particle from the interface into one of the two liquid phases) is strong, much larger than thermal energy even for nano-sized particles. For spherical particles, this free energy of adsorption is defined as [21]

$$
\Delta F_{a d s}=-\pi R^{2} \gamma_{12}\left(1-\left|\cos \theta_{2}\right|\right) .
$$

Additionally, according to experimental observations, adsorbed solid particles do not have surface activity as surfactant molecules often do [21]; therefore, partially wetted solid particles results in immobile interfaces. It should also be noted that interface localization can also occur even if the surface coating of the solid particle is miscible with one of the liquid phases if Relation (2-55) is satisfied; though, chain entanglement may give rise to other effects that make Relation (2-55) invalid [22].

The thermodynamic model discussed in this section is based on the assumption that the nanoparticles are fully dispersed to primary particles and fully isolated from one another; no particle-particle interactions are included. Deviations from the thermodynamic predictions of this section are expected for nanoparticle aggregates or non-isolated nanoparticles. For 
example, another assumption of the model is that the spherical nanoparticle has no porosity; however, tightly bound aggregates of nanoparticles would contain inter-particle porosity for which capillary action is an important factor during wetting and dewetting of liquids. Furthermore, particle-particle collisions introduce the energy of cohesion between the nanoparticles, which can be strong; this is the source of flocculation in solid-filled colloidal systems.

\subsubsection{Kinetic Effects}

Kinetic effects in polymer processing are often more influential than thermodynamics in governing the behavior of nanoparticle-filled polymer blends. The high viscosity and viscoelasticity of polymer melts are the influencing factors that make that so. The order of mixing is very important in dictating the final morphology and phase localization of nanoparticles in the blends [24-26]. The kinetic effects along with other influential effects are reviewed by Fenouillot et al. [23]. Further discussion of these effects are offered in Chapter 4. 


\section{Experimental Methods}

\subsection{Materials}

\subsubsection{Polymers}

Several immiscible polymer blend systems were investigated in this work. The desired attributes of the blend system were that the polymers be immiscible and preferably incompatible and that the viscosity ratio between them be high, thereby requiring extensional flows in blending to promote good mixing. The blends investigated were the following: 1) polycarbonate (PC) dispersed in styrene-co-acrylonitrile (SAN), 2) polystyrene (PS) dispersed in linear low-density polyethylene (LLDPE), and 3) high-density polyethylene (HDPE) dispersed in PS. PC/SAN blends are successful commercial engineering thermoplastic blends. Alternatively, PS and polyethylene (PE) are incompatible commodity plastics and require more efforts in compatibilization or mixing intensity to yield useful properties. PC/SAN and PS/LLDPE were preliminary blends of investigation. Detailed characterization and results of extensional flow blending of the unfilled preliminary blends are given in Sections 4.1.1 and 4.1.2, and the results of the incorporation of nanoparticles into these preliminary blends are presented in Sections 4.3.2 and 4.3.3. The primary blend of interest for this dissertation is HDPE/PS, where the grade of PS has a lower molecular weight than that of the grade of PS used in the PS/LLDPE blend system. The detailed characterization of HDPE/PS is given in Section 4.1.3, the results of extensional flow blending of the unfilled HDPE/PS are presented in Section 4.2, and the results of the incorporation of nanoparticles into the HDPE/PS blend are given in Section 4.3.4.

\subsubsection{Preliminary Blends PC/SAN and PS/LLDPE}

For the PC/SAN blend, the highest molecular weight polycarbonate produced commercially by Bayer MaterialScience (now Covestro), Makrolon ${ }^{\circledR} 3208$, was used. It is an amorphous thermoplastic with a density of $1.20 \mathrm{~g} / \mathrm{cm}^{3}$ and is typically processed at $240-300^{\circ} \mathrm{C}$. SAN 572 from GE Plastics and DMN 100 from Lanxess are research grades of SAN with similar viscosities and were used interchangeably. SAN is an amorphous copolymer with a density of about $1.07 \mathrm{~g} / \mathrm{cm}^{3}$ and is usually processed at $210-260^{\circ} \mathrm{C}$. 
For the blend termed "PS/LLDPE," PS 500 a general-purpose polystyrene grade from Total Petrochemicals was used. PS 500 is an amorphous thermoplastic with a density of 1.04 $\mathrm{g} / \mathrm{cm}^{3}$ and can be processed at $180-270^{\circ} \mathrm{C}$. The LLDPE grade used was Petrothene ${ }^{\circledast} \mathrm{GA} 574-000$ from LyondellBasell, which has a density of $0.926 \mathrm{~g} / \mathrm{cm}^{3}$ and processing temperature window of $170-230^{\circ} \mathrm{C}$.

\subsubsection{Primary Blend HDPE/PS}

Several other polystyrene and polyethylene grades were also characterized based on their viscosities in search of a blend system with high viscosity ratio for all shear rates expected during processing. These grades are further discussed in the Section 4.1 which gives the rheology data obtained for all of the grades acquired. The final blend system decided upon for intensive study was termed "HDPE/PS" and consisted of HDPE from NOVA Chemicals, grade NOVAPOL ${ }^{\circledR}$ HB-W355-A, dispersed in a developmental grade of general-purpose polystyrene from Americas Styrenics, grade name PS XU36305. The density of HDPE HBW355A is 0.955 $\mathrm{g} / \mathrm{cm}^{3}$, and its high load melt index (HLMI), measured at $190^{\circ} \mathrm{C} / 21.6 \mathrm{~kg}$, is $3.0 \mathrm{~g} / 10 \mathrm{~min}$.

The concentration of the dispersed polymer phase in the blend compositions varied from 1-25 wt\%. The terminology used to describe the composition of the binary polymer blends is to list the concentration in wt\% of the dispersed phase with the dispersed phase listed first followed by the matrix phase. For example, the blend of 5 wt\% HDPE/95 wt\% PS is referred to as "5\% HDPE/PS." Much of the work was done with 5 wt\% of the dispersed phase to attempt to minimize the amount of coalescence occurring, which is directly proportional to the concentration of the dispersed phase, and to focus on drop deformation and breakup. The binary polymer blends without nanofillers are referred to as "unfilled blends." The materials are further characterized in the Section 4.1, including literature surface tension values and the calculated interfacial tensions for the blend systems.

\subsubsection{Nanomaterials}

The nanoparticles considered in this work were the Aerosil ${ }^{\circledR}$ fumed silica grades provided by Evonik Industries (formerly Degussa). They are produced in a pyrolysis process 
which yields very pure amorphous $\mathrm{SiO}_{2}(>99.8 \%)$ [27]. The material consists of spherical primary particles with low porosity and size on the order of 5-40 $\mathrm{nm}$. The primary particles collide and fuse at high temperatures during manufacture, resulting in tightly bound aggregates of size 100-200 nm; these are also often referred to as mass fractals and may be characterized by fractal geometries. Furthermore, the as-received material is agglomerated with sizes up to tens of $\mu \mathrm{m}$, but these agglomerates are held together by van der Waals forces and are relatively easily dispersed down to aggregates [28-29]. Various grades with different hydrophobic surface treatments were provided, with more hydrophobic fumed silicas being more easily dispersed and resulting in better breakup of agglomerates. The grades used in this work range from untreated, hydrophilic Aerosil 200 to several different hydrophobic grades with surface treatments by organosilanes and even polydimethylsiloxane (PDMS). They are described in Table 3-1. The density of the solid particles was $2.20 \mathrm{~g} / \mathrm{cm}^{3}$. Further information and characterization of the nanosilica grades are given in Section 4.3.1. The blends filled with nanosilica are referred to in this work as "nano-filled blends." The nanosilica concentrations used depended on the mixing strategies employed, which will be described in Section 3.3.1.2. Typical polymer pellets and nanosilica powder are shown in Figure 3-1. 
Table 3-1: Fumed silica grades used in this work

\begin{tabular}{|c|c|c|}
\hline $\begin{array}{c}\text { Fumed } \\
\text { nanosilica } \\
\text { grade }\end{array}$ & $\begin{array}{c}\text { BET specific } \\
\text { surface area } \\
\qquad\left(\mathrm{m}^{2} / \mathrm{g}\right)\end{array}$ & Surface/surface-treating molecule \\
\hline Aerosil 200 & $200 \pm 25$ & No treatment; Si-OH moieties make surface hydrophilic \\
\hline Aerosil R974 & $170 \pm 20$ & Dimethyldichlorosilane (DDS) \\
\hline Aerosil R816 & $190 \pm 20$ & Hexadecylsilane $\left(\mathrm{C}_{16}\right)$ \\
\hline Aerosil R104 & $150 \pm 25$ & Octamethylcyclotetrasiloxane (OMCTS or D4) \\
\hline Aerosil R208 & $115 \pm 25$ & $\begin{array}{l}\text { Polydimethylsiloxane (PDMS); 4.5-6.5\% C content for } \\
\text { total nanoparticle }\end{array}$ \\
\hline Aerosil R812S & $220 \pm 25$ & Hexamethyldisilazane (HMDS or HMDZ) \\
\hline Aerosil R8200 & $160 \pm 25$ & Hexamethyldisilazane (HMDS or HMDZ) \\
\hline
\end{tabular}

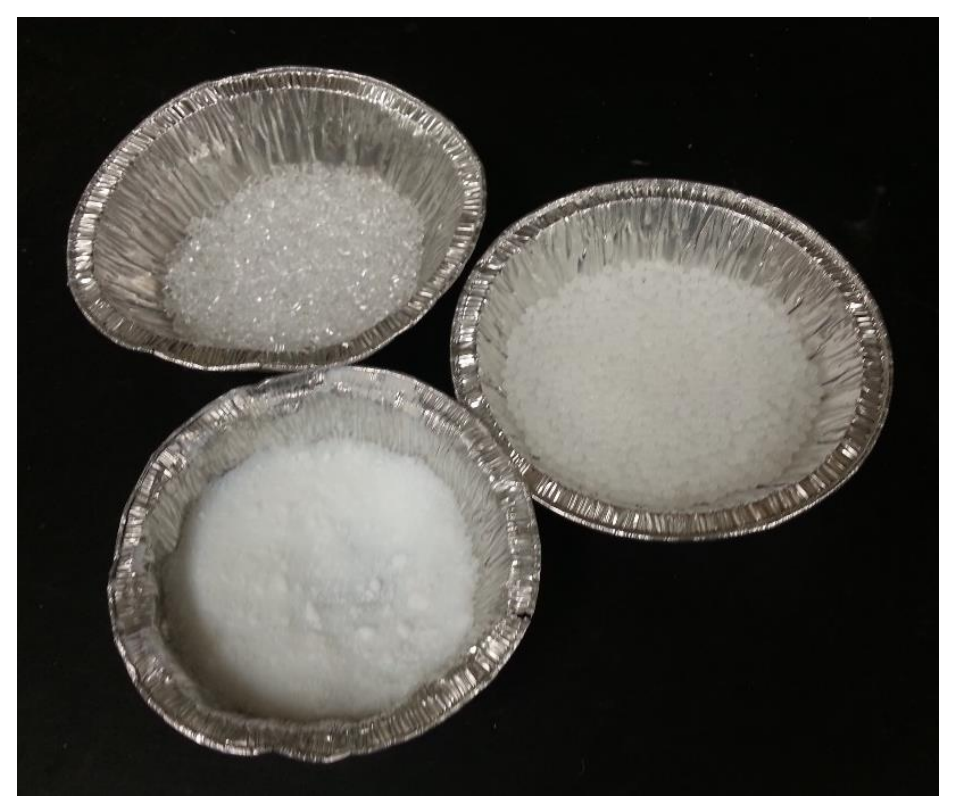

Figure 3-1: PS and HDPE pellets and nanosilica powders, clockwise from the top-left. 


\subsection{Materials Characterization Procedures}

The most important material property of interest was viscosity. Blends of high viscosity ratio were desired to emphasize the need for extensional flow fields to achieve good mixing. Polymer material suppliers usually cite a polymer's melt flow index (MFI) as a measure of the material's shear viscosity. A Dynisco LMI Series 4000 Melt Indexer, owned by the Polymer Characterization and Processing laboratory of Professor Rakesh K. Gupta of the Department of Chemical and Biomedical Engineering, was used when necessary to check the MFI of the materials being used. The indexer consists of a heated barrel, in which a small amount (a few grams) of dried pellets are loaded and melted and a piston with a weight is set on top of the molten material to force the melt to flow through a capillary at the bottom of the barrel. The extrudate is collected over a certain amount of time and weighed and converted to units of $\mathrm{g} / 10 \mathrm{~min}$. This is a relatively simple quality control technique that does not require much sample that can elucidate significant changes in molecular weight via changes in viscosity, but MFI values do not give a good estimation of a material's flow behavior in actual polymer melt processing at greater pressures and flow rates. The MFI is a single-point viscosity measurement at a single, relatively low shear rate, and a single temperature. More informative are full viscosity curves as measured by melt rheometers. Note that a method [30] is available for generating shear viscosity vs. shear rate curves from MFI via master curves established for various materials, but these are considered order-of-magnitude estimates and should not be used for detailed design or processing determinations.

For the other rheology measurements, the melt temperatures investigated were those within the normal processing temperature window for the materials. For PC, this is typically $250-340^{\circ} \mathrm{C}$; for SAN, $200-270^{\circ} \mathrm{C}$; and therefore for PC/SAN, $250-270^{\circ} \mathrm{C}$. For general-purpose PS (GPPS), this is typically $180-220^{\circ} \mathrm{C}$; for polyethylene (PE), $180-280^{\circ} \mathrm{C}$; and therefore for PS/LLDPE and HDPE/PS, $180-220^{\circ} \mathrm{C}$. The primary decision factor for the melt temperature of interest for each of the three blends was the viscosity ratio; the melt temperatures of investigation were those that yielded the largest viscosity ratio for each blend. 


\subsubsection{Parallel-Plate Rheology}

To measure the viscosities and other rheological properties of the materials used in this work, a parallel-plate rheometer and a capillary rheometer were used. The parallel-plate rheometer was a Rheometrics RMS-800, owned by the Polymer Characterization and Processing laboratory of Professor Rakesh K. Gupta of the Department of Chemical and Biomedical Engineering, with 25.0 mm-diameter plates; it is shown in Figure 3-2. This was used to measure the shear viscosity at low shear rates and to measure the dynamic rheology of the materials, including storage and loss moduli and phase angle. To prepare samples for the parallel-plate rheometer, material was compression molded into plaques using a PHI SQ-230H heated hydraulic press at melt temperature typically for 2-3 min at up to 15 ton of force using rectangular molds with thickness of 2-3 $\mathrm{mm}$. Around 1 inch square or circular samples were cut from the plaques and loaded in the rheometer and melted. The gap setting was 0.4 to $1.4 \mathrm{~mm}$. Tests were started after 5 min pre-test melting time. Shear rate sweeps were done at various melt temperatures over a shear rate range of $0.01-100 \mathrm{~s}^{-1}$. The measureable shear rate is limited at the low end by the sensitivity of the torque transducer which requires at least $2 \mathrm{~g} \cdot \mathrm{cm}$ of torque for accurate measurement, and additionally tests require much more time at very low shear rates. The shear rate is limited at the high end by the machine which can generate a max shear rate of $100 \mathrm{~s}^{-1}$ and is also limited by inertia which may cause material to release out radially from between the plates at high shear rates. 


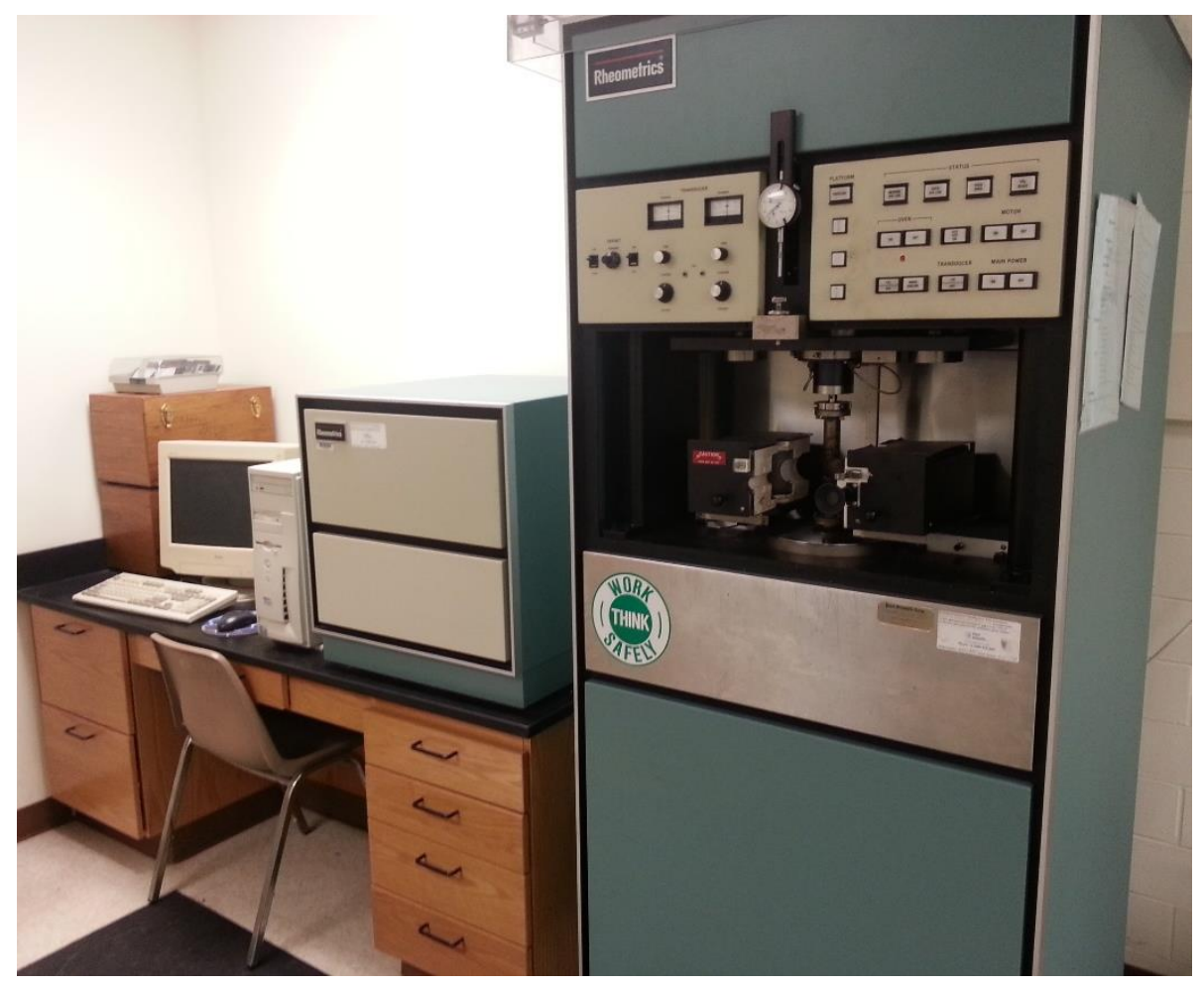

Figure 3-2: Rheometrics RMS-800 rotational rheometer

Dynamic rheology was also performed using the parallel-plate rheometer. The linear viscoelastic (LVE) region was first found using a strain sweep at $1 \mathrm{rad} / \mathrm{s}$ frequency. The LVE region is the range of shear strains for which the rheological properties have constant values, most importantly the storage and loss moduli, $G^{\prime}$ and $G^{\prime \prime}$, while also generating enough torque for accurate measurements. Then, dynamic strain-controlled frequency sweeps could be performed at shear strains in the LVE region, typically $2-10 \%$ strain and most commonly $5 \%$ strain. The frequency sweeps measured the complex viscosity, $\eta^{*} ; G^{\prime} ; G^{\prime \prime} ;$ and the tangent of the phase angle, $\tan \delta$, over a range from $0.1-100 \mathrm{rad} / \mathrm{s}$.

\subsubsection{Capillary Rheology}

The shear rates of interest in the processing of this work were much higher than $100 \mathrm{~s}^{-1}$. To measure the shear viscosity at higher shear rates, a Bohlin Instruments Rosand $\mathrm{RH} 2000$ capillary rheometer, owned by the Polymer Characterization and Processing laboratory of Professor Rakesh K. Gupta of the Department of Chemical and Biomedical Engineering, shown 
in Figure 3-3, was used. For this, about $35 \mathrm{~g}$ of dried pellets are loaded into a heated barrel and melted and a piston forces the melt through a capillary die at the bottom of the barrel. A pressure transducer measures the melt pressure just above the die, and the piston speed and therefore flow rate is precisely controlled. A pre-test melting procedure was used where the piston compresses the pellets at $100 \mathrm{~mm} / \mathrm{min}$ piston speed to $1 \mathrm{MPa}$, the material melts for 3 $\mathrm{min}$, the piston compresses again to $2 \mathrm{MPa}$, and the temperature is held for another $2 \mathrm{~min}$. Three capillary dies were used to measure viscosity, all of which with a length-to-diameter ratio of 16 and $90^{\circ}$ entry profile, meaning the top of the die was shaped as a converging cone with straight walls and a total cone angle of $90^{\circ}$ and therefore half cone angle of $45^{\circ}$. The three dies had diameters of $1.0,1.5$, and $2.0 \mathrm{~mm}$.

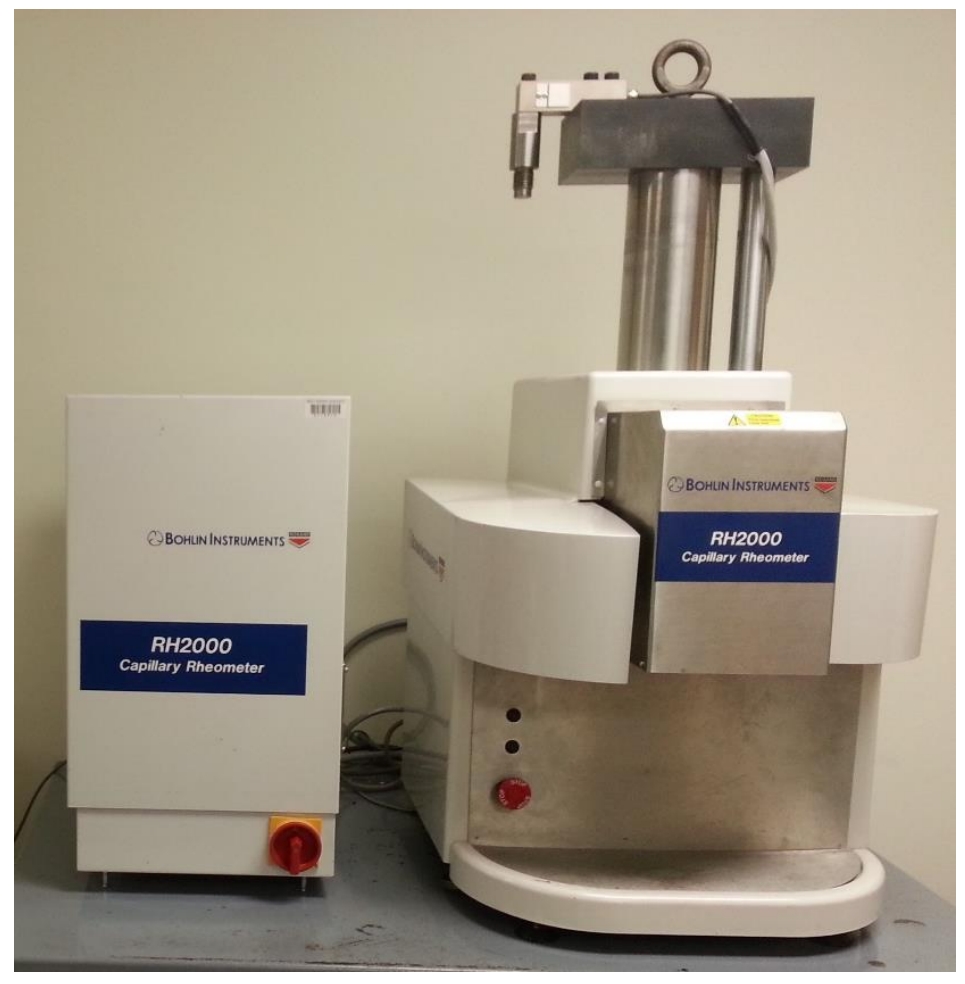

Figure 3-3: Bohlin Rosand RH2000 capillary rheometer

Typically, the $2 \mathrm{~mm}$-diameter, $32 \mathrm{~mm}$-length die was used for lower shear rates, from about $10-500 \mathrm{~s}^{-1}$, and the $1 \mathrm{~mm}$-diameter, $16 \mathrm{~mm}$-length die was used to measure the highest shear rates possible, for a range of about $500-10,000 \mathrm{~s}^{-1}$. The range of shear rates measureable is limited by the finite volume of material that may be loaded into the barrel and is also limited 
at the low end by the time required for the flow to reach a steady state and at the high end by the maximum piston force of the machine of $19 \mathrm{kN}$ and maximum pressure of $64 \mathrm{MPa}$ (both piston force and pressure include a safety factor and are $95 \%$ of the machine's actual maximums) and also by gross melt fracture of the extrudate which causes pressure readings to oscillate. No correction procedures were used to correct for end-effects (Bagley correction) or non-parabolic velocity profile (Weissenberg-Rabinowitsch correction) [31] because good agreement was found between the shear viscosity data collected using the parallel-plate and capillary rheometers without considering the correction factors. This is likely because the length-to-diameter ratio of 16 is sufficiently high to disregard entry and exit effects into and out of the capillary tube; ASTM D3835-16 suggests the use of a length-to-diameter of 20 for this reason.

\subsubsection{Differential Scanning Calorimetry}

The thermal properties of HDPE, PS, and 5\% HDPE/PS blends with various processing were also measured using differential scanning calorimetry (DSC). Samples of 6-9 mg were loaded in aluminum pans and loaded in a TA Instruments Q100 DSC, owned by the Polymer Characterization and Processing laboratory of Professor Rakesh K. Gupta of the Department of Chemical and Biomedical Engineering. The first heating and cooling procedure was to heat at $10^{\circ} \mathrm{C} / \mathrm{min}$ to $250^{\circ} \mathrm{C}$ and remain isothermal for $5 \mathrm{~min}$, then cool at $10^{\circ} \mathrm{C} / \mathrm{min}$ back to $25^{\circ} \mathrm{C}$. The first heating curve can reveal information on the thermal history of the materials. The glass transition temperature, $T_{\mathrm{g}}$, of PS was able to be measured. For HDPE, the melting temperature, $T_{\mathrm{m}}$, and crystalline heat of fusion for melting, $\Delta H_{\mathrm{m}}$, from which crystallinity can be calculated by dividing by the $\Delta H_{\mathrm{m}, \infty}$ for $100 \%$ crystalline HDPE were measured from the first heating. 


\subsection{Blending Procedures}

The materials were first pre-mixed together in relatively low-intensity blending operations to form what were referred to as "coarse" blends, meaning the dispersed-phase morphology was expected to be coarse compared to that of the blends after compatibilization or intensive blending operations. To study the effects of extensional flow on the morphology, custom dies were fabricated in the WVU Chemical and Biomedical Engineering machine shop using an electric-motor metal lathe, a numerically controlled (NC) mill, and a drill press. The fabricated dies incorporated various converging geometries to impart the blends to extensional flow fields. The dies are referred to as extensional flow cells (EFCs). Coarse blends of unfilled and nanosilica-filled blends were prepared and processed through extensional flow.

\subsubsection{Preparation of Coarse Blends}

Materials were dried thoroughly before any melt processing. PC and SAN were dried in an oven at $80-85^{\circ} \mathrm{C}$ for at least $5 \mathrm{hr}$. All other polymers were various grades of polyethylene or polystyrene, both of which are made by addition polymerization and do not necessarily require drying before processing. These were still dried in an oven, however, at $75^{\circ} \mathrm{C}$ for $2-4 \mathrm{hr}$. Nanosilica was dried at $100^{\circ} \mathrm{C}$ for $2-4 \mathrm{hr}$.

Coarse blends were prepared using the Haake PolyDrive internal mixer, owned by the Polymer Characterization and Processing laboratory of Professor Rakesh K. Gupta of the Department of Chemical and Biomedical Engineering, shown in Figure 3-4. The internal mixer used counter-rotating roller rotors to mix the materials in a batch process. The mixer also has real-time data collection of temperatures, rotor rotation rate, and torque on the rotors, which is an indication of the viscosity of the batch. Batch sizes were such that the internal cavity of the internal mixer was $70 \%$ full, which is recommended for optimal mixing. In this text, if sampling location is not specified, samples were taken from the inner space between the rotors. Samples were also taken from the outer area between the rotor and the cavity wall and in initial experiments from the material that adheres to the feed piston. The samples were allowed to cool through convection in air and conduction to the metal table on which they were 
placed. After internal mixing, the blended material was scraped from the rotors and cavity walls with a brass spatula and, after cooling and solidifying, broken into sizes suitable for additional processing by cutting with snips, granulating in a Dynisco Mini bench-top granulator, or granulating in a Fritsch Pulverisette 19-25 cutting mill (granulator), both owned by the Polymer Characterization and Processing laboratory of Professor Rakesh K. Gupta of the Department of Chemical and Biomedical Engineering,.

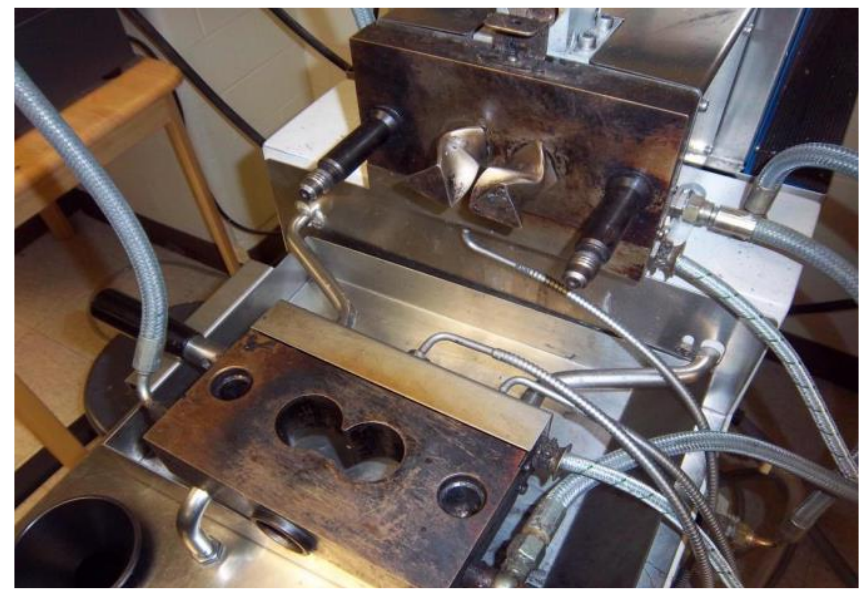

Figure 3-4: Internal mixer with roller rotors (Reproduced with permission from Finniss 2014 [32]).

\subsubsection{Unfilled Coarse Blends}

Unfilled polymer blends, i.e. those without nanosilica, were prepared by adding the polymer pellets together at once in the mixer. The concentration of the dispersed phase for some of the most used unfilled blend compositions in this work are given in Table 3-2 on both by weight and by volume bases. 
Table 3-2: Dispersed-phase concentration conversions for selected blend compositions

\begin{tabular}{|c|c|c|}
\hline Blend* & $\begin{array}{l}\text { Concentration } \\
\text { (wt\%) }\end{array}$ & $\begin{array}{l}\text { Concentration } \\
\text { (vol\%) }\end{array}$ \\
\hline \multirow[t]{2}{*}{ PC/SAN } & 5 & 4.5 \\
\hline & 25 & 22.9 \\
\hline PS/LLDPE & 15 & 13.6 \\
\hline HDPE/PS & 5 & 5.4 \\
\hline
\end{tabular}

*The convention of this text is to list the dispersed phase first followed by the matrix phase.

$\mathrm{PC} / \mathrm{SAN}$ coarse blends were mixed at $250^{\circ} \mathrm{C}$ with a rotor speed of $60 \mathrm{rpm}$ for $5 \mathrm{~min}$. This was confirmed to be sufficient mixing time as discussed in Section 4.1.1.3. Since a large amount of testing was planned for unfilled 5\% PC/SAN and unfilled 25\% PC/SAN, larger batches of those coarse blends were also prepared on a Leistritz Micro-27 twin screw extruder (TSE) at $250^{\circ} \mathrm{C}$ melt temperature in the die with co-rotating screw speeds of $30 \mathrm{rpm}$. The extrudate was cooled in a water bath and pelletized.

PS/LLDPE coarse blends were mixed in the internal mixer at $200^{\circ} \mathrm{C}, 80 \mathrm{rpm}, 6 \mathrm{~min}$, with samples removed from the inner section of the cavity between the roller rotors. A similar mixing analysis was done on 15\% PS/LLDPE blends and is discussed in Section 4.1.2.3.

Additionally, an extruded coarse blend was prepared on the TSE with $205^{\circ} \mathrm{C}$ melt temperature, $7 \mathrm{~kg} / \mathrm{h}$ total mass flow rate, and $75 \mathrm{rpm}$ rotation speed, generating $43 \%$ of the maximum torque of the motor. HDPE/PS coarse blends were mixed in the internal mixer at $220^{\circ} \mathrm{C}, 80 \mathrm{rpm}, 6 \mathrm{~min}$.

\subsubsection{Nano-Filled Coarse Blends}

Various mixing strategies were employed for mixing nanosilica into the polymer blends. The general blend composition and a high-level characterization of the grades of nanosilica used are presented in Figure 3-5. The material is a fine, white powder that aerosolizes when pouring into the internal mixer, so care had to be taken to maintain safety as well as minimize material losses. Figure 3-1 shows the polymer pellets and nanosilica powder before loading into the internal mixer. The temperatures and roller rotor rotation rates were identical to those for the unfilled coarse blends. The overall loading of nanosilica in the blends was typically 1,2 , or 4 wt\% up to $7 w t \%$. The convention used in this text to describe the compositions of the 
filled blends was to list the concentration of nanosilica in the entire blend and the concentration of the dispersed polymer phase with respect to the binary polymer system only. Therefore, a blend with an overall loading of $1 \mathrm{wt} \%$ nanosilica in a polymer binary system consisting of $5 \%$ HDPE/PS is referred to as "1\% nanosilica in 5\% HDPE/PS."

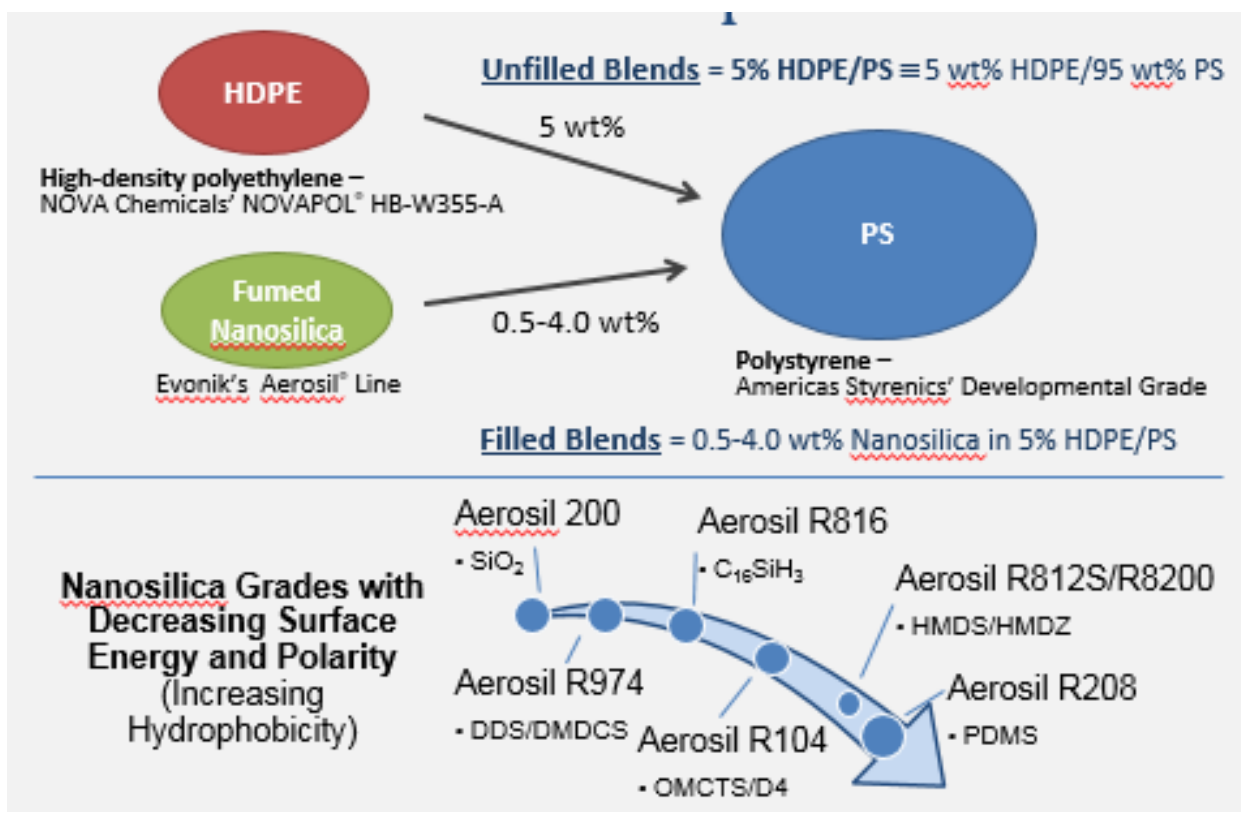

Figure 3-5: High-level schematic of blend compositions and nanosilica grade characteristics

Two different mixing methods were used: one-step mixing and two-step mixing. For one-step mixing, all components were added to the internal mixer at the same time. This mixing method was adapted, however, for the nano-filled HDPE/PS blends, in which the two polymers were first melted and premixed for $1 \mathrm{~min}$, then nanosilica was added and mixed for typically 6-10 min but up to 45 min. For two-step mixing, the nanosilica was first loaded into one of the two polymer phases, then the second polymer was added and mixed in a subsequent step. The nanosilica may be added first to the dispersed polymer phase or to the matrix phase. The typical procedure for two-step mixing of nano-filled HDPE/PS blends was to first prepare a masterbatch (MB) of nanosilica/HDPE at $220^{\circ} \mathrm{C}$ and $80 \mathrm{rpm}$ for at least $10 \mathrm{~min}$, then the concentrated nanosilica/HDPE MB was mixed with PS in a second melt-processing step at $220^{\circ} \mathrm{C}$ and $80 \mathrm{rpm}$ for typically $6-10 \mathrm{~min}$ but up to $45 \mathrm{~min}$. The MB composition was such 
that the final blend would consist of 0.5-4.0 wt\% nanosilica in 5\% HDPE/PS: 9.1 wt\% nanosilica/HDPE to produce a 0.5\% nanosilica in 5\% HDPE/PS final blend; 16.8 wt\% nanosilica/HDPE to produce a $1 \%$ nanosilica final blend; and $29.0 \mathrm{wt} \%$ nanosilica/HDPE to produce a $2 \%$ nanosilica in $5 \%$ HDPE/PS final blend.

Table 3-3: Concentration conversions for nano-filled 5\% HDPE/PS blends

\begin{tabular}{|c|c|c|c|c|}
\hline Blend step & $\begin{array}{l}\text { Nanosilica } \\
\text { concentration } \\
\text { (wt\%) }\end{array}$ & $\begin{array}{l}\text { HDPE } \\
\text { concentration } \\
\text { (wt\%) }\end{array}$ & $\begin{array}{l}\text { Nanosilica } \\
\text { concentration } \\
\text { (vol\%) }\end{array}$ & $\begin{array}{l}\text { HDPE } \\
\text { concentration } \\
\text { (vol\%) }\end{array}$ \\
\hline \multirow{4}{*}{$\begin{array}{l}\text { Final blend (one- } \\
\text { step mixing or } \\
\text { after second step } \\
\text { of two-step } \\
\text { mixing) }\end{array}$} & 0.50 & 4.98 & 0.24 & 5.43 \\
\hline & 1.00 & 4.95 & 0.48 & 5.42 \\
\hline & 2.00 & 4.90 & 0.96 & 5.39 \\
\hline & 4.00 & 4.80 & 1.93 & 5.34 \\
\hline \multirow{3}{*}{$\begin{array}{l}\text { First step of two- } \\
\text { step mixing } \\
\text { (Nanosilica/HDPE } \\
\text { MB) }\end{array}$} & $\begin{array}{l}9.13 \text { (for } 0.5 \% \text { in } \\
\text { final blend) }\end{array}$ & 90.87 & 4.18 & 95.82 \\
\hline & $\begin{array}{l}16.81 \text { (for } 1 \% \text { in } \\
\text { final blend) }\end{array}$ & 83.19 & 8.06 & 91.94 \\
\hline & $\begin{array}{r}28.99 \text { (for } 2 \% \text { in } \\
\text { final blend) }\end{array}$ & 71.01 & 15.05 & 84.95 \\
\hline
\end{tabular}

\subsubsection{Extensional Flow Blending}

The coarse materials (both unfilled and nano-filled) were exposed to strong extensional flow fields using in-house fabricated dies, or EFCs, that attached to the bottom of the capillary rheometer of Figure 3-3. Several different converging geometries were investigated with various entry profiles and nested obstructions that created multiple stretching episodes in one EFC. The EFCs are described in the following sections. The materials were dried before blending, then about $35 \mathrm{~g}$ of the granulated coarse material was loaded into the preheated barrel. The same pre-test packing procedure was used as that for the capillary rheology measurements described in Section 3.2.2. The PC/SAN blends were extensional flow-blended at 250 or $270^{\circ} \mathrm{C}$. Most PS/LLDPE blends were blended at $200^{\circ} \mathrm{C}$ with one experiment at 180 and 
$220^{\circ} \mathrm{C}$. HDPE/PS blends were blended at $220^{\circ} \mathrm{C}$. The EFCs were heated through thermal conduction from contact with the bottom of the capillary rheometer barrel.

The piston forced the material through the EFCs with piston speeds of $10-300 \mathrm{~mm} / \mathrm{min}$. With the barrel exit diameter of $14.5 \mathrm{~mm}$, the volumetric flow rates, $Q$, for the extensional flow blending were $2-50 \mathrm{~cm}^{3} / \mathrm{min}$, as reported in Table 3-4. A pressure transducer just before the die measured the pressure drop across the die, taking atmospheric pressure to be zero, and the piston force was also reported in real time. With a safety factor included, the maximum pressure and force for the machine were $64 \mathrm{MPa}$ and $19 \mathrm{kN}$, respectively. These, along with the finite volume of the barrel and the time required for a steady state to be reached for a given flow rate, limited the maximum flow rates for each EFC and material combination. Melt fracture also occurred at high flow rates and was reported when observed. Surface rippling and gross melt fracture with the extrudate corkscrewing out of the die are caused by different sources including fluid elasticity and slip at the walls, but the onset of melt fracture generally occurs at a wall shear stress on the order of $10^{5} \mathrm{~Pa}$ [33]. Therefore, the onset of melt fracture gives an indication of the wall shear stress. After pressure and force stabilized for a given flow rate, samples were taken from the extrudate strands. For PC/SAN and most PS/LLDPE blends, the strands were allowed to cool through convection in air and conduction to the metal surface onto which the strand was extruded, and for HDPE/PS blends, the strands were quenched in water about 9 inches below the exit of the capillary rheometer.

Table 3-4: Flow rates for extensional flow blending
\begin{tabular}{|r|r|}
\hline $\begin{array}{l}\text { Piston speed } \\
(\mathrm{mm} / \mathrm{min})\end{array}$ & $\begin{array}{l}\text { Volumetric flow } \\
\text { rate }\left(\mathrm{cm}^{3} / \mathrm{min}\right)\end{array}$ \\
\hline 10 & 2 \\
\hline 50 & 8 \\
\hline 100 & 17 \\
\hline 200 & 33 \\
\hline 300 & 50 \\
\hline
\end{tabular}

The EFCs fabricated in-house for extensional flow blending are referred to as converging cone, orifice, disk-in-tube, hemisphere-in-cone, and disk + hemisphere dies. The dies were machined from carbon steel in the WVU Chemical and Biomedical Engineering machine shop. They are described in the next sections. 


\subsubsection{Converging Cone Dies}

The primary converging dies, or extensional flow cells (EFCs), used in this work to investigate the effects of extensional flow blending on the morphology of the immiscible polymer blends were converging cone dies. Two converging cone dies with straight walls and half-cone angles of about $47^{\circ}$ were fabricated and are shown schematically in Figure 3-6. The dies were made from bolts with screw channels that allowed it to be screwed into the bottom of the capillary rheometer. The diameter of the tubular section at the entrance of the die was $11.7 \mathrm{~mm}$. Two dies were made with exit diameters of $0.559 \mathrm{~mm}$ and $0.330 \mathrm{~mm}$, equating to $\varepsilon=$ 6.1 and $\varepsilon=7.1$, respectively. The length of the straight-walled exit capillary tube section at the exit of each die was kept as short as possible to minimize the shear-flow contributions and maximize the extensional-flow contributions to the overall flow profile through the dies. This is what differentiates the fabricated converging cone dies from the standard rheometrymeasurement dies for the capillary rheometer. The straight-walled length of the exit capillarytube exits of the converging cone dies were both about $0.87 \mathrm{~mm}$, which equates to length-todiameter ratios of 1.6 and 2.6, respectively, for the converging cone dies with $0.559 \mathrm{~mm}$ and $0.330 \mathrm{~mm}$ exit diameters.

In the converging cone dies, the entry profile into the small-diameter section of the die is dictated by the entry profile of the die. This constrained geometry then prevents the formation of secondary flows if the cone angle of the die is less than that of the freely converging material. This is referred to as constrained convergence. 


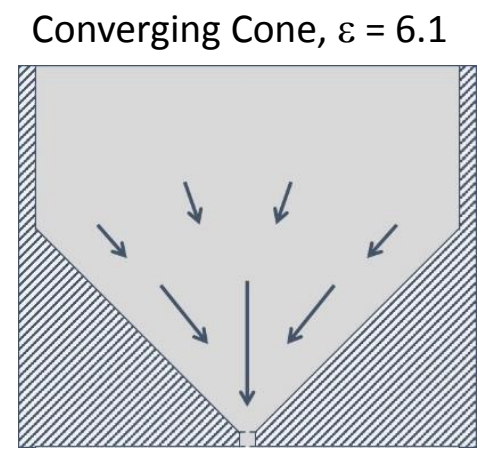

Converging Cone Die

Measurements

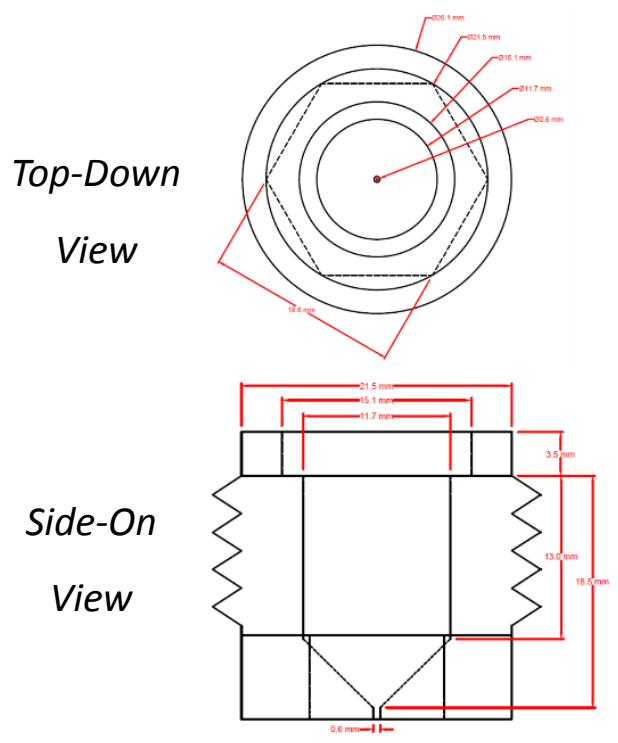

Figure 3-6: Schematic representations of the converging cone dies. Top-left - Converging cone with larger exit diameter of $0.559 \mathrm{~mm}$, equating to $\varepsilon=6.1$; Top-right - Converging cone with smaller exit diameter of $0.330 \mathrm{~mm}, \varepsilon=7.1$; Bottom-center-Top-down and side-on views of converging cone die with measurements.

\subsubsection{Orifice Dies}

Orifice dies consist of an abrupt constriction of cross-sectional area for flow, i.e. an orifice, where instead of the constricted convergence controlled by the walls of the converging cone dies, the polymer melt is free to flow in its own streamlines in unconstricted convergence. These orifice dies are described in a capillary rheometry as dies having $180^{\circ}$ entry profile, that is the top of the die is flat. Two orifice dies were made with two different orifice diameters: 
$0.559 \mathrm{~mm}$ and $0.330 \mathrm{~mm}$. The dies, shown in Figure 3-7, were loaded into the bottom of the capillary rheometer using the set screw used for typical capillary dies. The diameter of the bottom of the capillary rheometer barrel is $14.5 \mathrm{~mm}$. This equates to an extensional strain, $\varepsilon=2 \ln \left(d_{\text {in }} / d_{\text {out }}\right)$, of 6.5 . The extensional strain for the orifice die with $0.330 \mathrm{~mm}$ orifice diameter is 7.6. The bottoms of the dies were bored to shorten the length of the smalldiameter capillary section at the entrance of the die. The purpose of this was to minimize the shear contribution in the die and focus on the generation of extensional flow fields.

Furthermore, this bore-out allowed the two dies to be stacked one atop the other and expose the material to two extensional, or stretching, episodes in one pass through the EFC. When the stacked configuration was used, the larger orifice diameter die was always stacked on top of the smaller orifice diameter die, and the bore-out section provided a diverging section. The resulting two stretching episodes have extensional strains of 6.5 and 6.0, respectively.

The orifice dies have free convergence, meaning the molten material is free to assume its own entry profile into the orifice. Secondary flows, or vortices, may form in the corners of the entry zone. The entry profile of the molten material is material- and flow rate-dependent. The orifice dies differed from the standard rheometry-measurement dies for the rheometer by their entry profile and length-to-diameter ratio of the exit, straight-walled capillary tube section, the latter of which was kept to a minimum by boring out the dies from down-stream side. The land length of the straight-walled exit capillary tube section of the orifice dies was about $0.5 \mathrm{~mm}$, yielding a length-to-diameter ratio of 0.9 and 1.5 , respectively, for the dies with $0.559 \mathrm{~mm}$ and $0.330 \mathrm{~mm}$ exit diameters. 

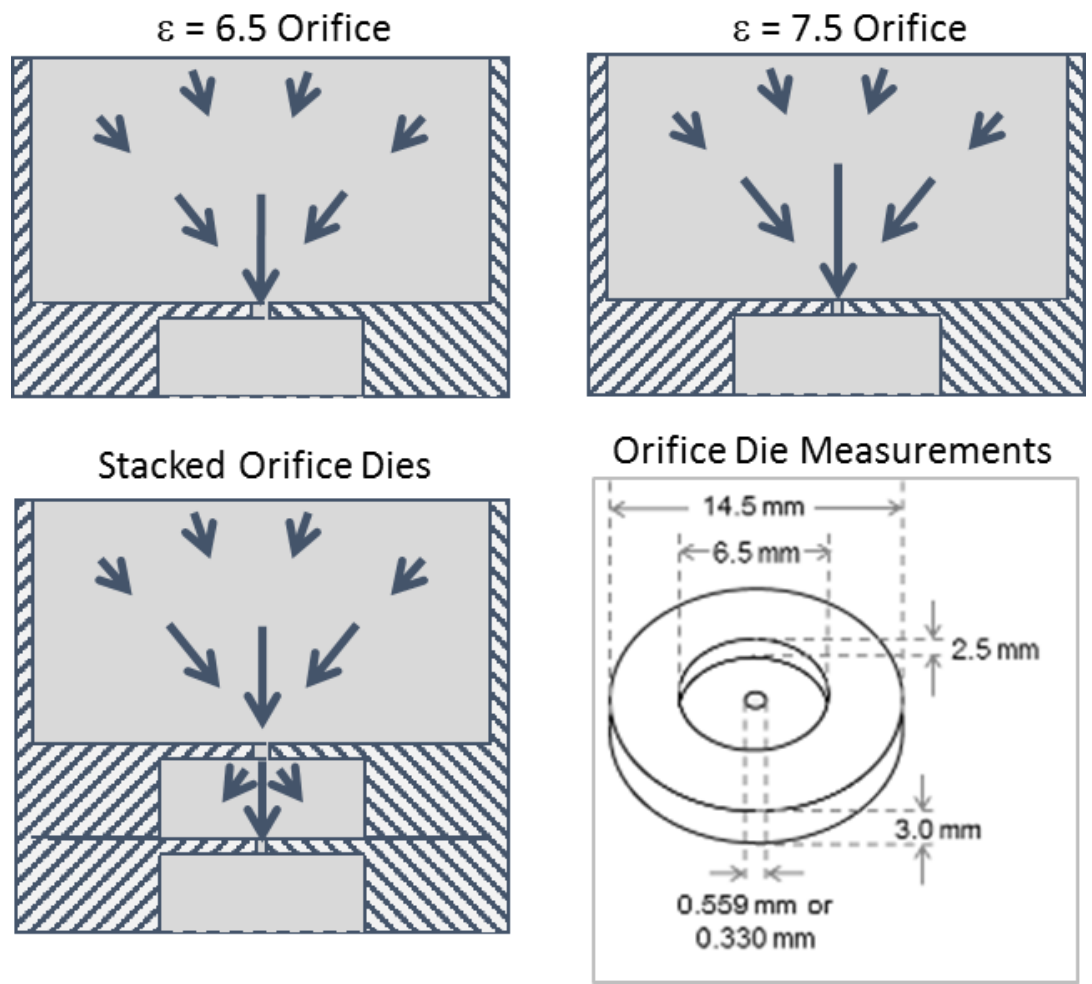

Figure 3-7: Schematic representations of the orifice dies. Top-left-Orifice with larger orifice diameter of $0.559 \mathrm{~mm}$, equating to $\varepsilon=6.5$; Top-right - Orifice with smaller orifice diameter of $0.330 \mathrm{~mm}, \varepsilon=7.6$; Bottom-left - Orifice dies stacked with larger orifice diameter die on top of smaller orifice die, $\varepsilon=6.5 \& 6.0 ;$ Bottom-right - Upside-down view of orifice die with measurements.

\subsubsection{Disk-in-Tube Die}

To add another stretching episode to the converging cone die, inserts were fabricated that nest within the converging cone die with the larger exit diameter, $\varepsilon=6.1$. The first of these inserts consisted of a disk that nested within the tubular section of the die. The disk-in-tube die is shown in Figure 3-8. The disk insert hung in place by resting on the shoulder of the entrance of the converging cone die, and sections were cut out of the top of the insert to allow the molten material to flow through. The disk was seated to the tube walls using an abrasive mixture, creating a gap between the disk insert and the tube wall of about 0.001 in or $25 \mu \mathrm{m}$. The diameter of the tube section of the converging cone die is $11.7 \mathrm{~mm}$, and that of the stem of the disk insert is $5.4 \mathrm{~mm}$. This equates to a cross-sectional area of $84.6 \mathrm{~mm}^{2}$. With a gap of 
0.001 in in the converging zone, the cross-sectional area in the convergence was $0.9 \mathrm{~mm}^{2}$. Therefore, the extensional strain of the stretching episode between the disk and tube wall, using $\varepsilon=\ln \left(A_{\mathrm{c}, \text { in }} / A_{\mathrm{c}, \text { out }}\right)$, was $\varepsilon_{1}=4.5$. After divergence, the stretching episode at the exit of the converging cone generates $\varepsilon_{2}=6.1$. The straight walls of the disk insert produce a large amount of shear as well, which will be discussed further in Section 4.2.3.

In this first iteration, the disk walls were straight and parallel to the tube wall, generating a large amount of shear in addition to the extensional flow at the entrance to the convergence. After passing through the gap between the disk and tube wall, a diverging section is present followed by a second stretching episode at the exit of the converging cone.

Disk-in-Tube Die Measurements

Disk-in-Tube Die

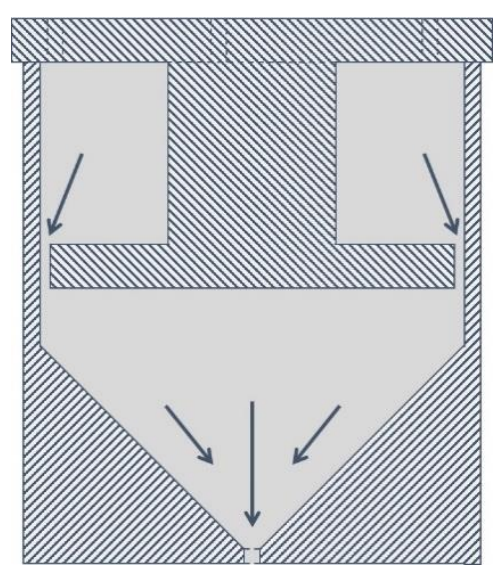

Disk Insert

Converging Cone,

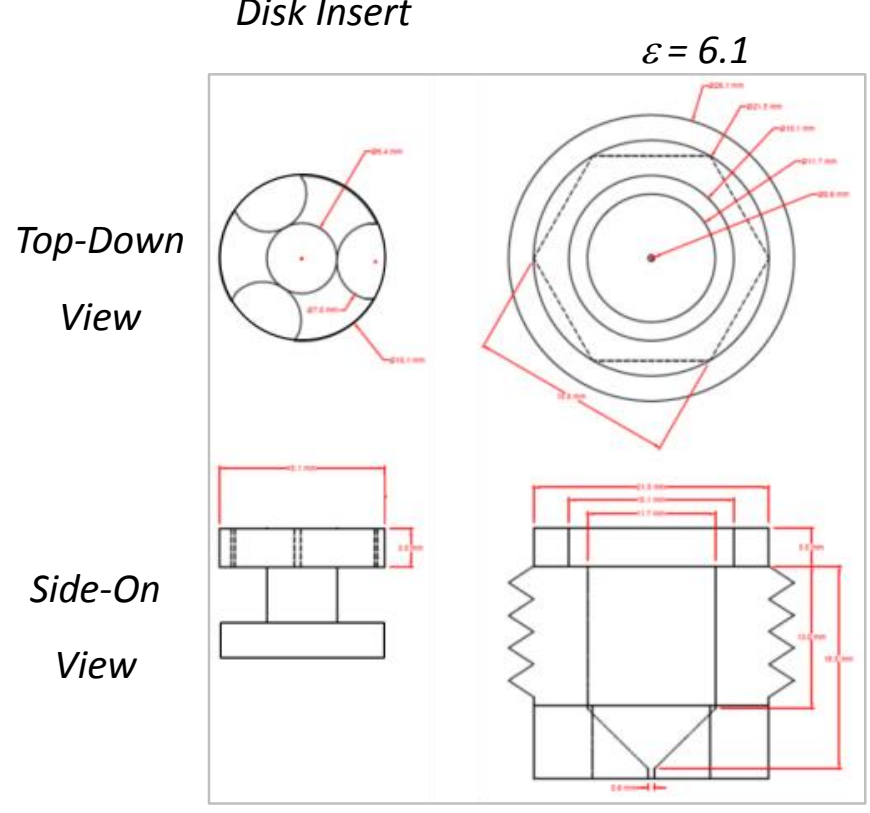

Figure 3-8: Schematic representations of the disk-in-tube die. Left - Schematic showing two stretching episodes; Right - Top-down and side-on views of disk-in-tube die with measurements of the disk insert and converging cone housing. 


\subsubsection{Hemisphere-in-Cone Die}

Another EFC design imparting two stretching episodes onto the material consists of an insert with a hemispherical or rounded end that nests within the converging cone section. This design was favorable for homogenization of oil-in-water emulsions in earlier work in the group [34]. The hemisphere-in-cone die, shown in Figure 3-9, has cut-outs for flow in the upper lip that rests on the shoulder at the top of the converging cone with $\varepsilon=6.1$, similar to the disk-intube die. However, unlike the disk-in-tube die for which the gap of the convergence cannot be changed, the gap of the converging zone between the hemisphere and cone wall can be set using steel shims of different thicknesses as spacers between the hemisphere insert and the shoulder of the converging cone die. Steel shims were acquired with thicknesses of 0.001 , 0.002 , and 0.004 in $(25.4,50.8$, and $101.6 \mu \mathrm{m})$, and the 0.004 in shims were also stacked using two or four shims. The spacer thickness then was varied from $0.001-0.016$ in $(0.025-0.406$ $\mathrm{mm})$.

Hemisphere-in-Cone

Die

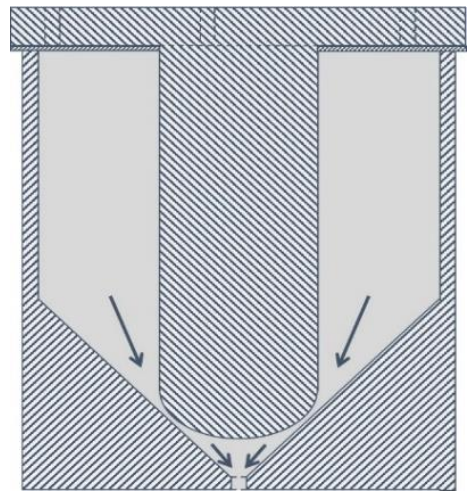

Hemisphere-in-Cone Measurements

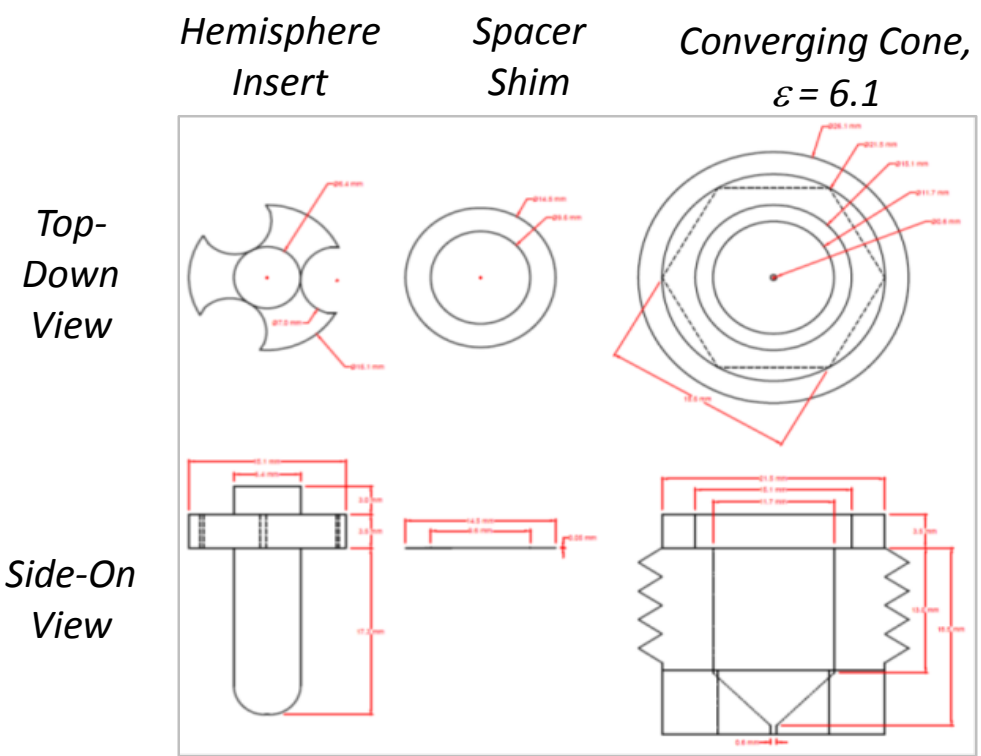

Figure 3-9: Schematic representations of the hemisphere-in-cone die. Left-Schematic showing two stretching episodes; Right - Top-down and side-on views of hemisphere-in-cone die with measurements of the hemisphere insert, spacer shim, and converging cone housing. 
The minimum area for flow in the convergence between the hemisphere and the converging cone wall is the conical frustum correlating to the minimum gap normal to the converging cone wall as depicted in Figure 3-10. The minimum gap, $G$, is set by the thickness of the spacers, $t_{\mathrm{sp}}$, and the cone half-angle, $\theta$. The radius $R_{1}$ is the location where the hemisphere insert touches the cone wall with no spacers added, and the radius $R_{2}$ corresponds to the bottom of the conical frustum for flow. The area $A_{1}$ for flow through the conical frustum is the following:

$$
A_{1}=\pi\left(R_{1}+R_{2}\right) G \text {. }
$$

The extensional strain through this first converging section is given in Table 3-5. The extensional strain through the second stretching episode is dependent on the decrease in crosssectional area from the circular cross section at $R_{2}$ and that at the exit of the converging cone. The second extensional strain is also given in Table 3-5. 


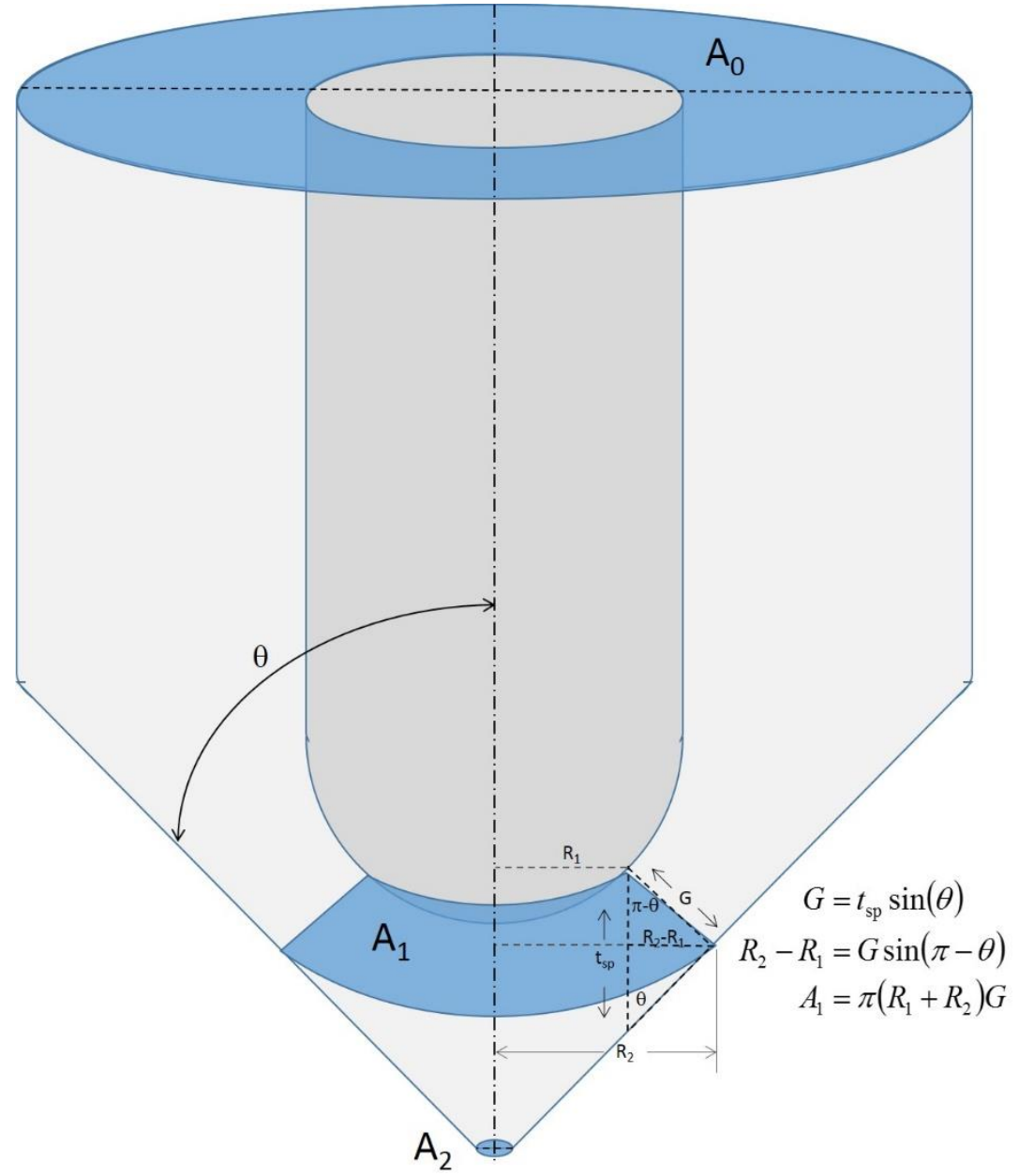

Figure 3-10: Cross-sectional areas for flow through hemisphere-in-cone die. The minimum gap, $G$, between the hemisphere insert and the cone wall is dependent on the thickness of the spacers, $t_{\mathrm{sp}}$, and the cone half-angle, $\theta$. Also shown are the equations for calculating the area for flow through the conical frustum between the hemisphere and the cone wall.

Table 3-5: Extensional strain through hemisphere-in-cone die

\begin{tabular}{|l|r|r|r|r|r|}
\hline Spacer thickness, $\boldsymbol{t}_{\text {sp }}(\mathrm{in})$ & 0.001 & 0.002 & 0.004 & 0.008 & 0.016 \\
\hline Spacer thickness, $\boldsymbol{t}_{\mathrm{sp}}(\boldsymbol{\mu m})$ & 25.4 & 50.8 & 101.6 & 203.2 & 406.4 \\
\hline Gap $(\mathbf{m m})$ & 0.019 & 0.037 & 0.074 & 0.148 & 0.296 \\
\hline $\boldsymbol{R}_{\mathbf{2}}(\mathbf{m m})$ & 2.450 & 2.463 & 2.488 & 2.539 & 2.640 \\
\hline $\boldsymbol{A}_{\mathbf{1}}=\boldsymbol{\pi}\left(\boldsymbol{R}_{\mathbf{1}}+\boldsymbol{R}_{\mathbf{2}}\right) \boldsymbol{G}\left(\mathbf{m m}^{\mathbf{2}}\right)$ & 0.284 & 0.570 & 1.145 & 2.315 & 4.723 \\
\hline $\boldsymbol{\varepsilon}_{\mathbf{1}}=\ln \left(\boldsymbol{A}_{\mathbf{0}} / \boldsymbol{A}_{\mathbf{1}}\right)$ & 5.7 & 5.0 & 4.3 & 3.6 & 2.9 \\
\hline $\boldsymbol{A}_{\mathbf{c}}\left(\boldsymbol{R}_{\mathbf{2}}\right)=\pi \boldsymbol{R}_{\mathbf{2}} \mathbf{2}\left(\mathbf{m m}^{\mathbf{2}}\right)$ & 18.9 & 19.1 & 19.5 & 20.3 & 21.9 \\
\hline $\boldsymbol{A}_{\mathbf{2}}\left(\mathbf{m m}^{\mathbf{2}}\right)$ & & & 0.439 & & \\
\hline $\boldsymbol{\varepsilon}_{\mathbf{2}}=\ln \left[\boldsymbol{A}_{\mathbf{c}}\left(\boldsymbol{R}_{\mathbf{2}}\right) / \boldsymbol{A}_{\mathbf{2}}\right]$ & 3.8 & 3.8 & 3.8 & 3.8 & 3.9 \\
\hline
\end{tabular}




\subsubsection{Disk + Hemisphere Die}

The last EFC fabricated for this work consisted of the hemisphere-in-cone die with an additional disk insert that wraps around the hemisphere insert, resulting in three stretching episodes for one pass through the EFC. This EFC is referred to as the "disk + hemisphere die" and is shown in Figure 3-11. The bottom of the disk insert has cut-outs for flow and rests on the top of the converging cone. The outer edge of the disk is tapered in this die to decrease the amount of shear compared to that of the disk-in-tube die. The minimum gap in this first stretching episode is nominally the same as that of the disk-in-tube die, resulting in the same total extensional strain through this first stretching episode. The extensional strain through the second stretching episode is set by spacer shims for the hemisphere insert, in the same way as the hemisphere-in-cone die, and the resulting extensional strains for the second and third stretching episodes are identical to those of the hemisphere-in-cone die, as given in Table 3-5.

$$
\text { Disk + Hemisphere Die }
$$

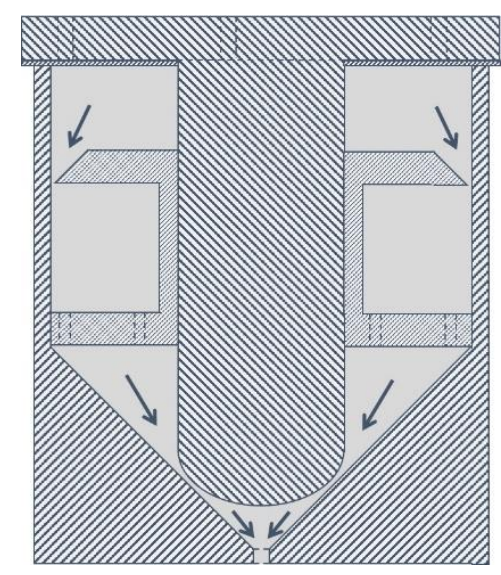

Figure 3-11: Schematic representation of the disk + hemisphere die. Additional disk insert adds third stretching episode. 


\subsection{Microscopy and Image Analysis}

\subsubsection{Sample Preparation}

The samples of coarse blends from the internal mixer or extruder and those from the extensional-flow mixed blends consisting of strand extrudate from the EFCs were analyzed microscopically. The samples for analysis with by scanning electron microscopy (SEM) were cryo-fractured by immersing in liquid nitrogen for at least 2 minutes then fracturing. Selective samples were also polished from the side with fine grit diamond abrasive paper under a flow of water to minimize frictional heating. Samples for analysis by transmission electron microscopy (TEM) were cryo-microtomed using fresh glass blades with liquid nitrogen cooling to about $60^{\circ} \mathrm{C}$ in $100 \mathrm{~nm}$ thick slices. The cryo-ultramicrotome used was owned and operated by the research laboratory of Professor Xueyan Song of the Department of Mechanical and Aerospace Engineering of WVU. Selective solvents were also sometimes used to selectively dissolve, or etch, one of the polymer phases to enhance contrast between the phases. For the PC/SAN blends, a $6 \mathrm{M}$ aqueous sodium hydroxide $(\mathrm{NaOH})$ solution was used to etch the dispersed PC phase from the surface of the sample. The time required for etching was analyzed and is discussed in Section 4.1.1. After etching, the samples were rinsed in deionized (d.i.) water for one day and dried in a vacuum desiccator. For PS/LLDPE blends, several organic solvents are available to etch the PS phase from the relatively chemical-resistant PE phase. In this work, dimethylformamide (DMF) was used, and the time required for etching was analyzed with one day found to be sufficient. Selectively dissolving HDPE from HDPE/PS blends is much more difficult. Polyolefins are soluble in refluxing xylenes, and some report that they are soluble in heated solutions of chromic and sulfuric acids. For safety reasons, this etching was not done for HDPE/PS blends. In one experiment, the PS matrix phase of HDPE/PS was dissolved using toluene.

For samples for SEM, an electrically conductive sample surface is required to prevent charging of the surface which disrupts microscopy. The sample surface was sputter coated with $60 \%$ gold/40\% palladium ( $\mathrm{Au} / \mathrm{Pd}$ ) using an SPI-MODULE sputter coater, owned by the 
Department of Chemical and Biomedical Engineering. The layer thickness of Au/Pd was about 8-24 nm.

\subsubsection{Microscopy}

Sputter-coated samples were imaged using a Hitachi S-4700 field-emission scanning electron microscope (FE-SEM), owned by the Shared Research Facilities of WVU, and operated by the author after training by Shared Research Facilities staff. Typical SEM parameters of $5 \mathrm{kV}$ accelerating voltage and $6 \mathrm{~mm}$ working distance were used for topological imaging for investigating the dispersed polymer phase morphology. Several images were taken at various magnifications for each sample to ensure both small and large dispersed polymer phase drops were included. For later work including all of that with HDPE/PS, standard magnifications were used for each sample along with several images at various magnifications. Both upper and lower detectors were used for many images, referred to as mixed detectors, and for later work, just the upper detector was used for detailed topology investigation.

The atomic composition of samples was also measured using energy dispersive $x$-ray spectroscopy (EDS), which was coupled with the SEM using a separate detector. For this, the accelerating voltage was $10-15 \mathrm{kV}$ and working distance was $12 \mathrm{~mm}$. Spot scans give a spectrum at a given spot, with spot size resolution on the order of microns. Line scans and 2D maps were also used. For maps, for a given element, pixels in the image are colored if the peak corresponding to that element is present in the spectrum.

TEM was used particularly to investigate the localization of nanosilica in the polymer blends. For this, the cryo-microtomed samples were imaged using a JEOL JEM-2100 transmission electron microscope, owned by the Shared Research Facilities of WVU and operated by Professor Xueyan Song. The typical TEM imaging parameters were $200 \mathrm{kV}$ accelerating voltage with appropriate adjustments to the focus to enhance the contrast between the polymer phases as well as the nanoparticle phase. The polymer samples were sensitive to the electron beam and only allowed for a short time for imaging before vaporizing. The TEM was also equipped with an EDS detector, and spot scans were performed. 


\subsubsection{Analysis}

Image analysis software, ImageJ, v. 1.45s, freely available for download online, was used to analyze the SEM images. Dispersed phase polymer drops were outlined, and the image was converted to a binary black-and-white image. From exported area measurements for each drop, $A_{i}$, an equivalent circle diameter, $d_{i}$, was calculated,

$$
d_{i}=\sqrt{\frac{4 A_{i}}{\pi}} .
$$

To describe the dispersed phase drop size and size distribution, the number-average diameter, $d_{\mathrm{n}}$, volume-average diameter, $d_{\mathrm{v}}$, and polydispersity index, P.I., were computed, as given by the following:

$$
\begin{gathered}
\bar{d}_{\mathrm{n}}=\frac{\sum_{i} n_{i} d_{i}}{\sum_{i} n_{i}} \\
\bar{d}_{\mathrm{v}}=\frac{\sum_{i} n_{i} d_{i}^{4}}{\sum_{i} n_{i} d_{i}^{3}} \\
\text { P.I. }=\frac{\bar{d}_{\mathrm{v}}}{\bar{d}_{\mathrm{n}}} .
\end{gathered}
$$

The number-average diameter, $d_{n}$, is representative of small drops in the blend, while the volume-average diameter, $d_{v}$, is sensitive to large drops in the blend. The polydispersity index, P.I., then serves as a metric for the width of the size distribution. Three replicates were analyzed for many samples with the average and standard deviation of each metric being used.

Discrete histograms of frequency vs. dispersed phase drop size were also plotted using Matlab, operated under the floating licenses held by the Department of Chemical and Biomedical Engineering of WVU. The curves were fitted to a log-normal distribution, and the log-normal fit was described by the geometric mean, $d_{G}$, which is equivalent to a count median diameter, and by the geometric standard deviation, $\sigma_{G}$, which are the exponential of the arithmetic mean and standard deviation. The skewness and kurtosis were also calculated for the raw data and fitted data. Skewness is the third moment of a distribution and quantifies the asymmetry of the distribution. Since particle size distributions are almost always skewed to the right, resulting in a positive skewness, whereas symmetric distributions have zero skewness and those skewed to the left have negative skewness. Kurtosis is the fourth moment and quantifies 
the relative weight of the tails of the distribution compared to that of the center of the distribution.

Using the procedure from [35], around 300 drops were needed to be analyzed for each sample to characterize the morphology. In this work, 11-13 SEM images having various magnifications were analyzed for each of three replicate samples, resulting in an average of at least 322 dispersed phase drops being analyzed per sample. 


\section{Results and Discussion}

\subsection{Selection of Blend System}

The objectives of this dissertation research, given in Section 1.3, were the following: 1) Characterize the effects of design variables related to extensional flow blending on the morphology of immiscible blends and develop general extensional flow cell (EFC) design considerations, 2 ) investigate the effects of nanoparticle stabilization on blend morphology, and 3) examine extensional flow blending with nanoparticle stabilization. For the purposes of Objective 1, the blend system must simply be immiscible, forming a two-phase mixture. To accentuate the effects of extensional flow blending, the blend should also have high viscosity ratio, the ratio of dispersed-phase viscosity to matrix-phase viscosity. These blends require extensional flows to promote good mixing. Several blend systems were investigated in this work and will be discussed in this section. Preliminary blend systems were PC/SAN and PS/LLDPE; their characterization and the results of extensional flow blending of their unfilled blends are presented in Sections 4.1.1 and 4.1.2, and the results of the incorporation of nanoparticles into these preliminary blends are presented in Sections 4.3.2 and 4.3.3. The primary blend of interest was HDPE/PS. Its characterization is given in Section 4.1.3, the results of extensional flow blending of its unfilled blends are presented in Section 4.2, and the results of the incorporation of nanoparticles into HDPE/PS are given in Section 4.3.4.

\subsubsection{Polycarbonate (PC)/Styrene Acrylonitrile (SAN)}

Blends of polycarbonate (PC) and styrene acrylonitrile (SAN) are technologically important blends used in the automotive, home appliance, consumer electronics, and industrial markets. PC and SAN are fairly compatible with one another and useful blends have been commercialized without the need for compatibilizers. PC and SAN is the basis for $\mathrm{PC} /$ acrylonitrile butadiene styrene (ABS) blends as well, where ABS consists of butadiene rubber particles with SAN chains grafted to the surface. In the case of blends consisting of PC dispersed in SAN, PC may serve as a toughening agent to the SAN matrix. The properties of 
such blends depend strongly on the blend morphology, however, which is the purpose of this investigation.

\subsubsection{Rheology of PC/SAN}

The viscosities of the PC and SAN grades, Makrolon 3208 and SAN 572, respectively, were measured using a parallel-plate rheometer and a capillary rheometer, as described in Section 3.2. The viscosity curves are given in Figure 4-1 along with the viscosity ratio as functions of shear rate and temperature. The curves at lower shear rates $\dot{\gamma}$ were measured using the parallel plate rheometer, for which shear rate is limited to $\dot{\gamma}<100 \mathrm{~s}^{-1}$ or even lower if material releases from between the plates due to inertia. The curves at higher shear rates were measured using the capillary rheometer, for which low shear rates are difficult to measure because of the time required for flow to reach a steady equilibrium state and high shear rates are limited by the barrel capacity and maximum pressure drop and piston force of the machine. Desired for this work was a blend system with viscosity ratio greater than 3.8 , the asymptote for pure shear mixing above which pure shear mixing cannot breakup dispersed-phase drops. The viscosity ratio of the PC/SAN blend exceeds 3.8 for $\dot{\gamma}>20 \mathrm{~s}^{-1}$. The shear rate in an internal mixer typically ranges from 1-400 $\mathrm{s}^{-1}$ [36], and so the viscosity ratio of this PC/SAN blend is lower than 3.8 for most of the shear rates generated in the internal mixer. Therefore, the internal mixer which generates predominantly shear flow fields may be able to produce adequate mixing. 


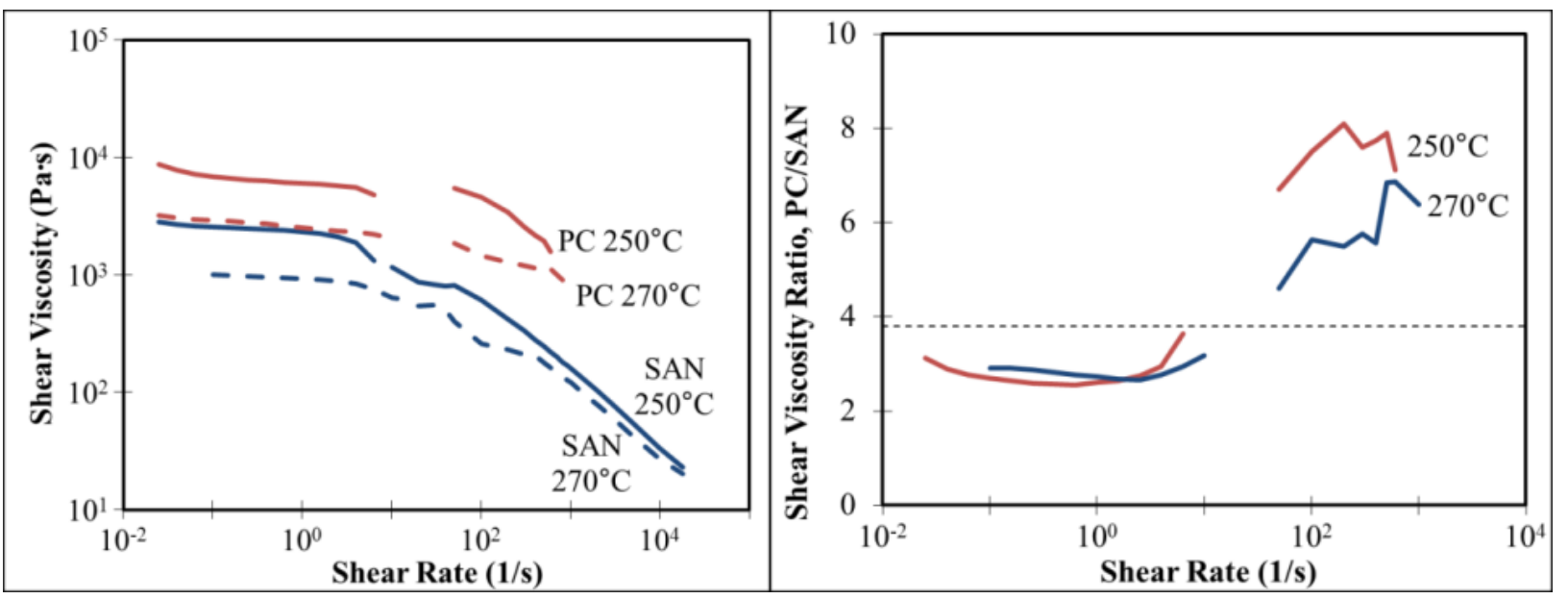

Figure 4-1: PC and SAN viscosities and blend viscosity ratio. Shear viscosities of PC and SAN at $250^{\circ} \mathrm{C}$ and $270^{\circ} \mathrm{C}$ measured with parallel-plate rheometer (low $\dot{\gamma}$ ) and capillary rheometer (high $\dot{\gamma})$. Viscosity ratio, $p$, exceeds 3.8 for $\dot{\gamma}>20 \mathrm{~s}^{-1}$.

Indeed, the morphology resulting from initial blending in the internal mixer was fairly fine with the size of the dispersed PC phase in the sub-micron range, which is considered fine in practice and typically yields satisfactory mechanical properties. A representative scanning electron microscope (SEM) image for the blend of 5 wt\% PC/95 wt\% SAN (hereinafter called 5\% $\mathrm{PC} / \mathrm{SAN}$ ) is shown in Figure 4-2. This is referred to as a coarse blend, in that it was not passed through extensional flow yet to promote dispersion. This blend was prepared on a twin-screw extruder at $250^{\circ} \mathrm{C}$ with $30 \mathrm{rpm}$ screw speed. Samples for SEM were prepared by cryogenically fracturing in liquid nitrogen then etching, or selectively dissolving, the PC dispersed phase from the fracture surface using a $6 \mathrm{M}$ sodium hydroxide $(\mathrm{NaOH})$ in water solution. The etching process was done to increase the phase contrast of the SEM images, in that the holes left behind where PC drops were dissolved appear darker than intact PC drops in the SAN matrix.

The amount of time required to completely etch the dispersed PC phase was determined by immersing samples in the $6 \mathrm{M} \mathrm{NaOH}$ solution for increasing amounts of time and finding the time at which the measured dispersed-phase size equalized. Size was determined using an automatic image processing using ImageJ software to measure the Feret's diameter, which is the maximum chord length that can be drawn across a given drop. Over 1000 drops per sample were measured for this experiment. As shown in Figure 4-3, the 
measured drop size increased as the polycarbonate dispersed phase was dissolved, until a stable size was reached after 3 days. This implies that 3 days are sufficient to dissolve all of the polycarbonate from the surface of the sample.

A procedure was investigated to correct the drop diameters based on areal measurements from planar sections through the sample to account for the true volume-wise drop diameter. One can imagine that planar sections through a sample may cut quasi-spherical drops at different places, not necessarily at the midplane of each drop to reveal the true drop diameter [37]. The procedure used was the Schwartz-Saltykov analysis [38] which uses the probability distribution of random planar sections of a sphere to create a table of coefficients used to calculate the average sphere diameter from circular, section diameters. The method was found to effectively decrease diameters obtained by about 15\%, as shown in Figure 4-3. This is consistent with previous work done in the group [39]. The Schwartz-Saltykov analysis did not affect the trends observed in this example or others and was not used in further analyses. This conclusion was also drawn by others [40-41] who further justified that Schwartz-Saltykov corrections are not necessary because one can imagine that planar sections are more likely to section larger drops than smaller drops but larger drops are also more likely to be located on the edge of the frame of images and therefore be excluded from analysis. 


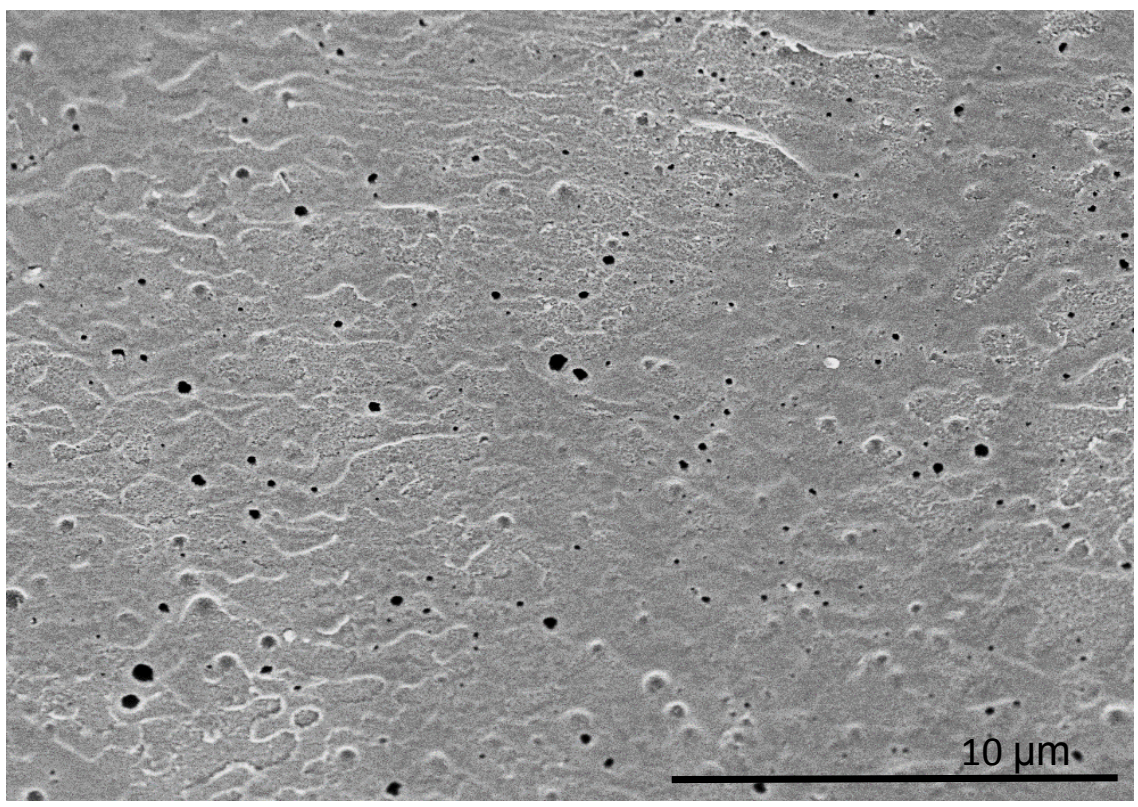

Figure 4-2: 5\% PC/SAN coarse blend. Coarse blend was made using twin screw extruder at $250^{\circ} \mathrm{C}$ and $30 \mathrm{rpm}$ screw speed.

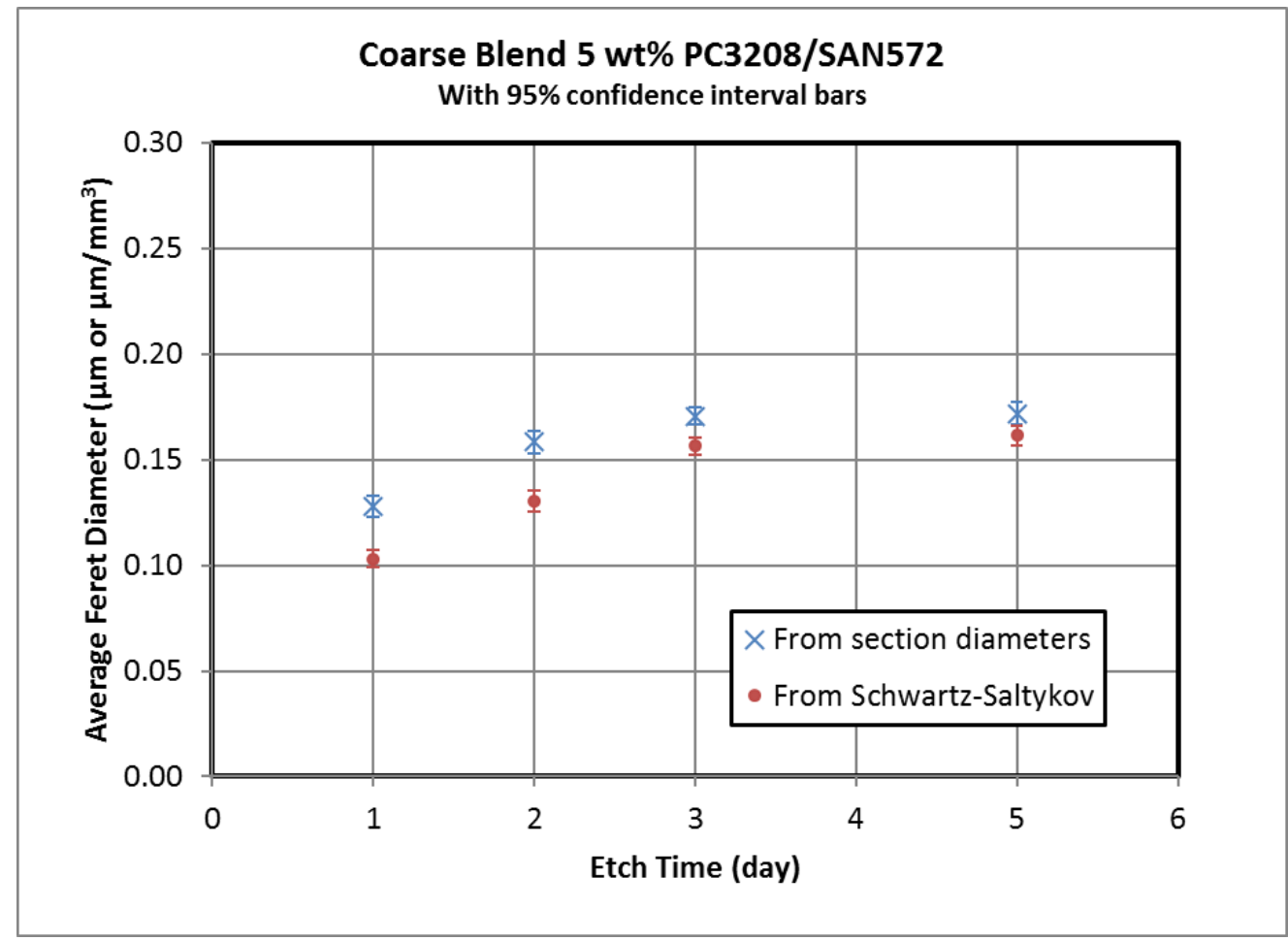

Figure 4-3: Etching PC dispersed phase in 5\% PC/SAN. Feret's diameter is the longest chord length across a drop. Error bars represent the 95\% confidence interval. Schwartz-Saltykov analysis is a procedure to correct measurements from 2D sections to 3D actual drop diameters. 


\subsubsection{Interfacial Tension of PC/SAN}

The morphology observed even for predominantly shear mixed coarse blends is fairly fine, with PC drops in the sub-micron size range, as seen in Figure 4-2. The reason for this is because the interfacial tension of PC/SAN is quite low for typical polymer blends, and the blend is even considered partially miscible in much of the literature [42-43]. This implies that the thermodynamic window for mutual solubility of PC and SAN is large with respect to many factors and that the interface phase (entanglement of molecules of each phase) is more pronounced than it is for most immiscible blends. The interfacial tension between PC and SAN at a melt temperature of about $260^{\circ} \mathrm{C}$ is about $1.4 \pm 1.1 \mathrm{mN} / \mathrm{m}$ [44-48]. This is quite low for typical immiscible polymer blends. The large standard deviation cited is a result of the inconsistencies of measured and calculated surface tension data using different methods and laboratories.

\subsubsection{PC/SAN Coarse Blend Mixing Time}

To determine the length of time required for the morphology to stabilize in the internal mixer during the preparation of the coarse blends, a batch of 1\% PC/SAN was blended in the internal mixer and samples were taken at various mixing times up to $20 \mathrm{~min}$. The results of this study are given in Figure 4-4. As shown, $d_{\mathrm{n}}$ increases from $1 \mathrm{~min}$ to $5 \mathrm{~min}$ and remains level thereafter, while $d_{v}$ stays fairly constant up to $10 \mathrm{~min}$, at which point it increases only to decrease again at $20 \mathrm{~min}$. The early increase in $d_{\mathrm{n}}$ was unexpected for this blend, but it may be the result of a small degree of flow-induced coalescence, which has been reported in the literature even at such a low dispersed-phase concentration. The behavior of $d_{v}$ is also unexpected. Samples in this experiment were collected by raising the plunger to the entry port of the mixing cavity and removing some of the molten material that adheres to the bottom of the plunger, which is typically about $1 \mathrm{in}^{3}$ of material in total. This was later identified to be an inconsistent sampling method, and subsequent coarse blend samples were taken from within the mixing cavity between the rotors by opening the cavity completely. Despite this potential source of experimental error, since typical internal mixing times for polymer blending are 5-10 min and studies have shown that the majority of mixing occurs in the first few minutes of 
melting and mixing [49] [50], and since $d_{\mathrm{n}}, d_{\mathrm{v}}$, and P.I. were all very consistent from 5 min to 10 $\mathrm{min}$, it was concluded that $5 \mathrm{~min}$ is sufficient mixing time in the internal mixer to produce a relatively stable morphology for PC/SAN blends.

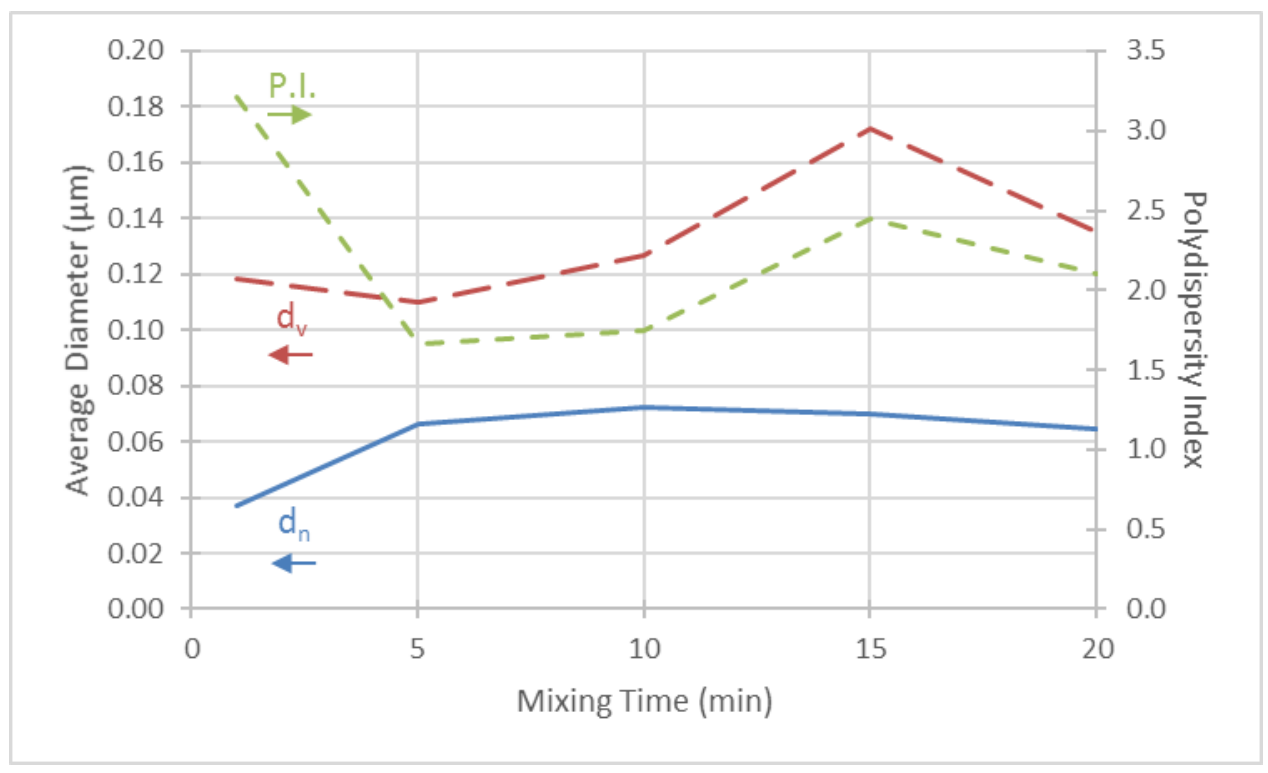

Figure 4-4: Effect of mixing time on morphology of $1 \% \mathrm{PC} / \mathrm{SAN}$. Mixing at $250^{\circ} \mathrm{C}$ with roller rotor speed of $60 \mathrm{rpm}$.

\subsubsection{Extensional Flow Blending of PC/SAN}

\subsection{PC/SAN through Orifice Dies}

To investigate the effects of extensional flow on the morphology of the PC/SAN blends, orifice dies were fabricated for use on the capillary rheometer, as discussed in Section 3.3.2.2. For orifice dies, available cross-sectional area for flow abruptly converges from the diameter of the capillary rheometer barrel (reservoir) to a small orifice diameter; this is referred to as a $180^{\circ}$ cone inlet. The tube length at the orifice diameter is minimized to avoid shear flow contributions to droplet dynamics and pressure drop. The orifice dies used in this work have $0.559 \mathrm{~mm}$ and $0.330 \mathrm{~mm}$ orifice diameters, respectively, and they may be used singly or in a stacked configuration, with the larger orifice-diameter die stacked on top of the smaller orificediameter die. 
For a given temperature and flow rate, increasing extensional strain is expected to result in decreasing dispersed phase size, and multiple stretching episodes are expected to further reduce the dispersed phase size. In general, this was found to be fairly true for flow of the $5 \%$ PC/SAN blend through the orifice dies. Representative scanning electron microscope (SEM) images are shown in Figure 4-5 for the coarse blend and that after extensional flow through the two different orifice dies and the dies in the stacked configuration with processing at $250^{\circ} \mathrm{C}$ and $17 \mathrm{~cm}^{3} / \mathrm{min}$. The morphology is quantified in Figure 4-6. Both the $d_{\mathrm{n}}$ and $d_{\mathrm{v}}$ decreased with extensional flow through the orifice dies, with each slightly larger for the $\varepsilon=7.6$ die compared to those for the $\varepsilon=6.5$ die. The spread of the dispersed phase size distribution, given by P.I., was slightly smaller though for flow through the $\varepsilon=7.6$ die. The average size and the P.I. decreased even further with flow through the stacked orifice dies, which is represented in Figure 4- 6 by an arbitrary extensional strain of 10 because flow through two stretching episodes with $\varepsilon_{1}=6.5$ and $\varepsilon_{2}=6.0$ is unlikely to have the same effect as flow through one episode with the same overall strain or with a strain equal to the sum of the two, $\varepsilon=\varepsilon_{1}+\varepsilon_{2}$. Flow through the stacked orifice dies resulted in about a factor of 2 decrease in size compared to the coarse blend. 

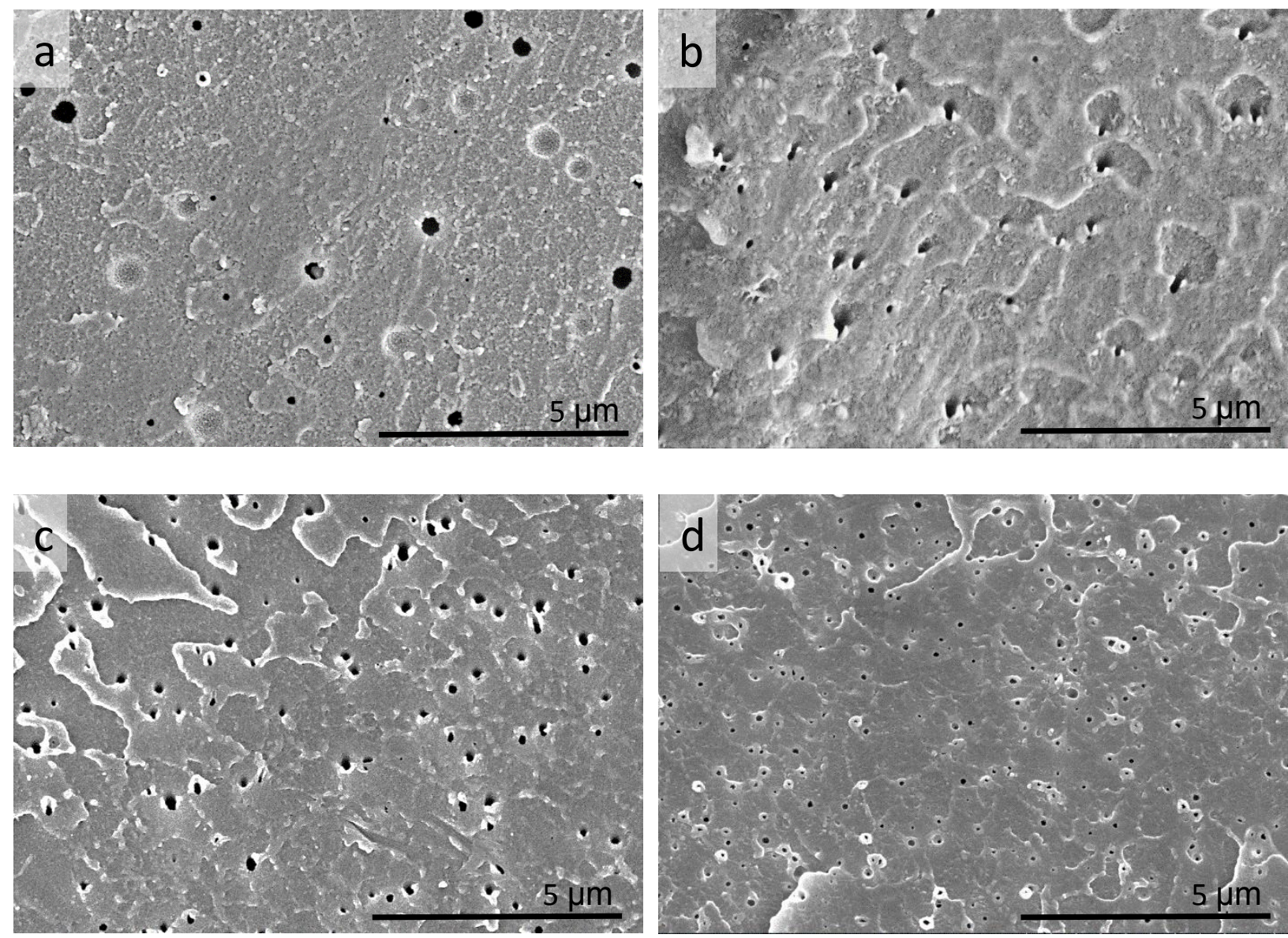

Figure 4-5: SEM of 5\% PC/SAN with various extensional strains through orifice dies.

Processed at $250^{\circ} \mathrm{C}$ and $17 \mathrm{~cm}^{3} / \mathrm{min}$ : a) Coarse blend, $\varepsilon=0 ; b$ ) Large-exit, $\left.\varepsilon=6.5 ; c\right)$ Small-exit, $\varepsilon$ $=7.6 ; d)$ Stacked, $\varepsilon=6.5 \&$ 6.0. Scale bar for all is $5 \mu \mathrm{m}$. 


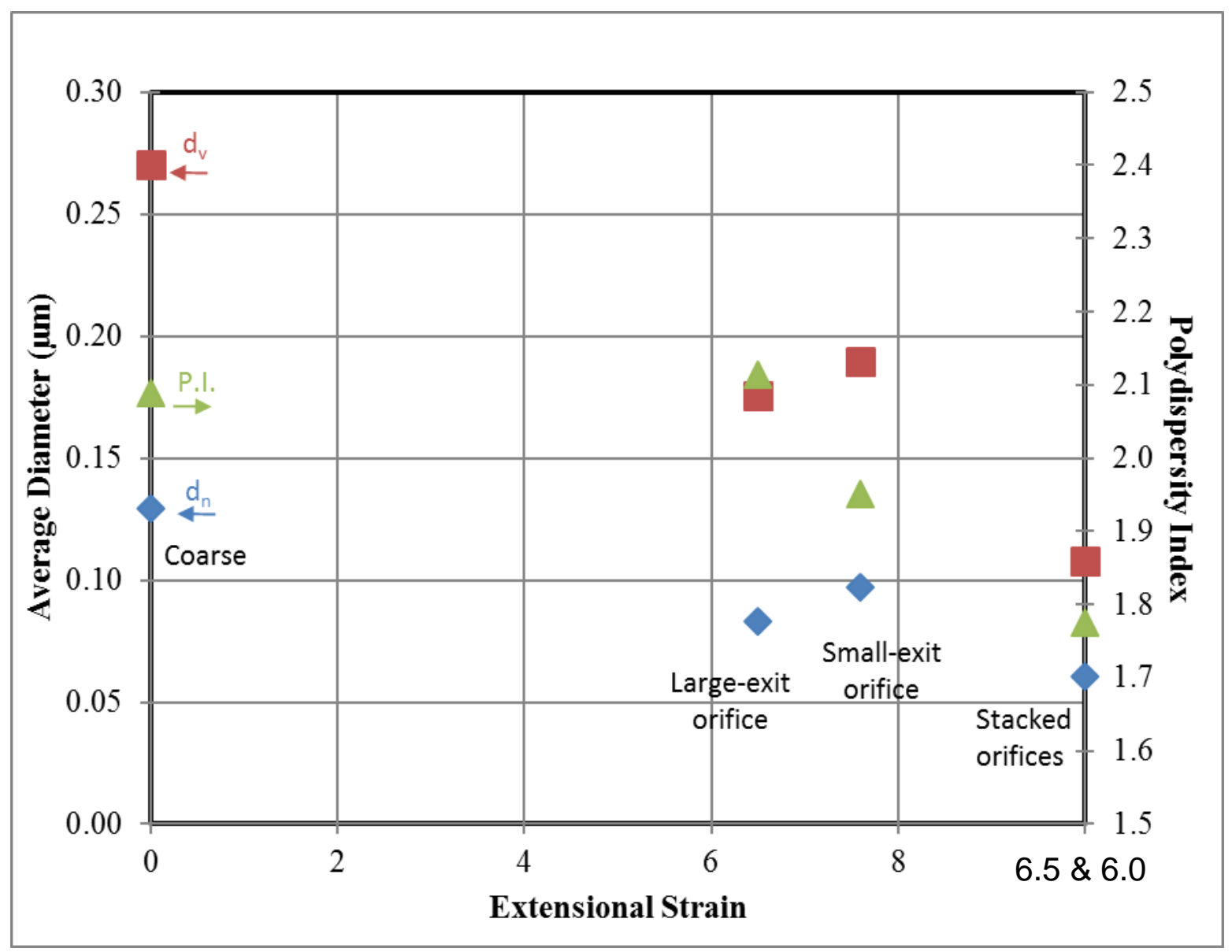

Figure 4-6: Effect of extensional strain for 5\% PC/SAN through orifice dies. Morphologies of coarse blend and blends passed through large-exit, small-exit, and stacked orifices at $250^{\circ} \mathrm{C}$ and $17 \mathrm{~cm}^{3} / \mathrm{min}$.

The results shown in Figure 4-7 and Figure 4-8 illustrate the effects of composition, temperature, total strain, and flowrate (proportional to strain rate) on the morphology of PC/SAN blends through the orifice dies. Blends of $5 \%$ and $25 \%$ PC/SAN were passed through the three orifice die configurations at $250^{\circ} \mathrm{C}$ and $270^{\circ} \mathrm{C}$ and at flowrates of 17 and $83 \mathrm{~cm}^{3} / \mathrm{min}$. Higher dispersed-phase concentration resulted in higher drop size, namely $d_{v}$, as exemplified by the differences in the scales of the ordinate axes for the plots for $5 \%$ PC/SAN and $25 \%$ PC/SAN in Figure 4-7. This is expected and is the result of increased coalescence, which is directly proportional to concentration. The number-average drop size $d_{\mathrm{n}}$ was not as significantly influenced by concentration, and therefore, larger P.I. resulted for $25 \%$ PC/SAN compared to $5 \% \mathrm{PC} / \mathrm{SAN}$ in Figure 4-8. This supports the fact that a blending is a dynamic process with both 
drop breakup and coalescence occurring; with increased dispersed-phase concentration, larger drops resulting from increased coalescence inflate $d_{v}$ and P.I., while small drops are still present due to drop breakup resulting in a relatively small $d_{n}$.

With increasing temperature, smaller dispersed-phase size is expected since the viscosity ratio is smaller for the blend at $270^{\circ} \mathrm{C}$ than it is at $250^{\circ} \mathrm{C}$, as shown in Figure $4-1$. Overall the resulting morphology was relatively insensitive to processing temperature, but surprisingly the size was slightly higher for many cases for $270^{\circ} \mathrm{C}$ compared to $250^{\circ} \mathrm{C}$. This may have been a result of increased coalescence due to lower viscosity resulting in higher phase mobility, since the observation seemed to have been more pronounced for the $25 \%$ blend compared to the $5 \% \mathrm{PC} / \mathrm{SAN}$ blend.

As expected, larger total extensional strains generally resulted in finer morphologies of the blends, while the size distribution, that is the spread of the range of sizes, stayed relatively constant with varying total strain. Multiple stretching episodes also generally decreased the phase size even further, with the finest morphologies resulting from flow through the orifice dies in the stacked configuration. These observations did not hold true, however, in a few cases for the $25 \% \mathrm{PC} / \mathrm{SAN}$ blend. Flow through the small orifice-diameter die with $\varepsilon=7.6$ and through the stacked dies may have actually had an increased amount of coalescence for this higher concentration blend. As shown in Figure 4-9, coalescence may still occur in converging extensional geometries due to converging, nonparallel streamlines and variable velocity resulting in drop collisions.

Increasing the flowrate from $17 \mathrm{~cm}^{3} / \mathrm{min}$ to $83 \mathrm{~cm}^{3} / \mathrm{min}$ generally resulted in coarser morphologies, that is $d_{v}$ was usually larger for the higher flowrate. The number-average diameter $d_{n}$ was generally slightly smaller for the higher flowrate, so the P.I. was larger for the higher flowrate. This result is not surprising because of the transient nature of drop dispersion. Drop stretching and breakup take time, and the elastic nature of polymers, with the highviscosity dispersed phase likely being more elastic as well, exacerbating that. The $83 \mathrm{~cm}^{3} / \mathrm{min}$ flowrate corresponds to a piston speed of $500 \mathrm{~mm} / \mathrm{min}$, where the maximum speed of the machine is $600 \mathrm{~mm} / \mathrm{min}$. This is a very fast flowrate with gross melt fracture occurring in the extrudate strand. This adverse effect of high flowrates is something to keep in mind in the 
design and operation of extensional flow cells. Extensional flow fields are generally very short lived, especially for those created in converging flows, as opposed to those in hyperbolic flows. Therefore, drops stretching into threads generally do not have enough time to reach an equilibrium length and width, so the process is by its nature very transient.

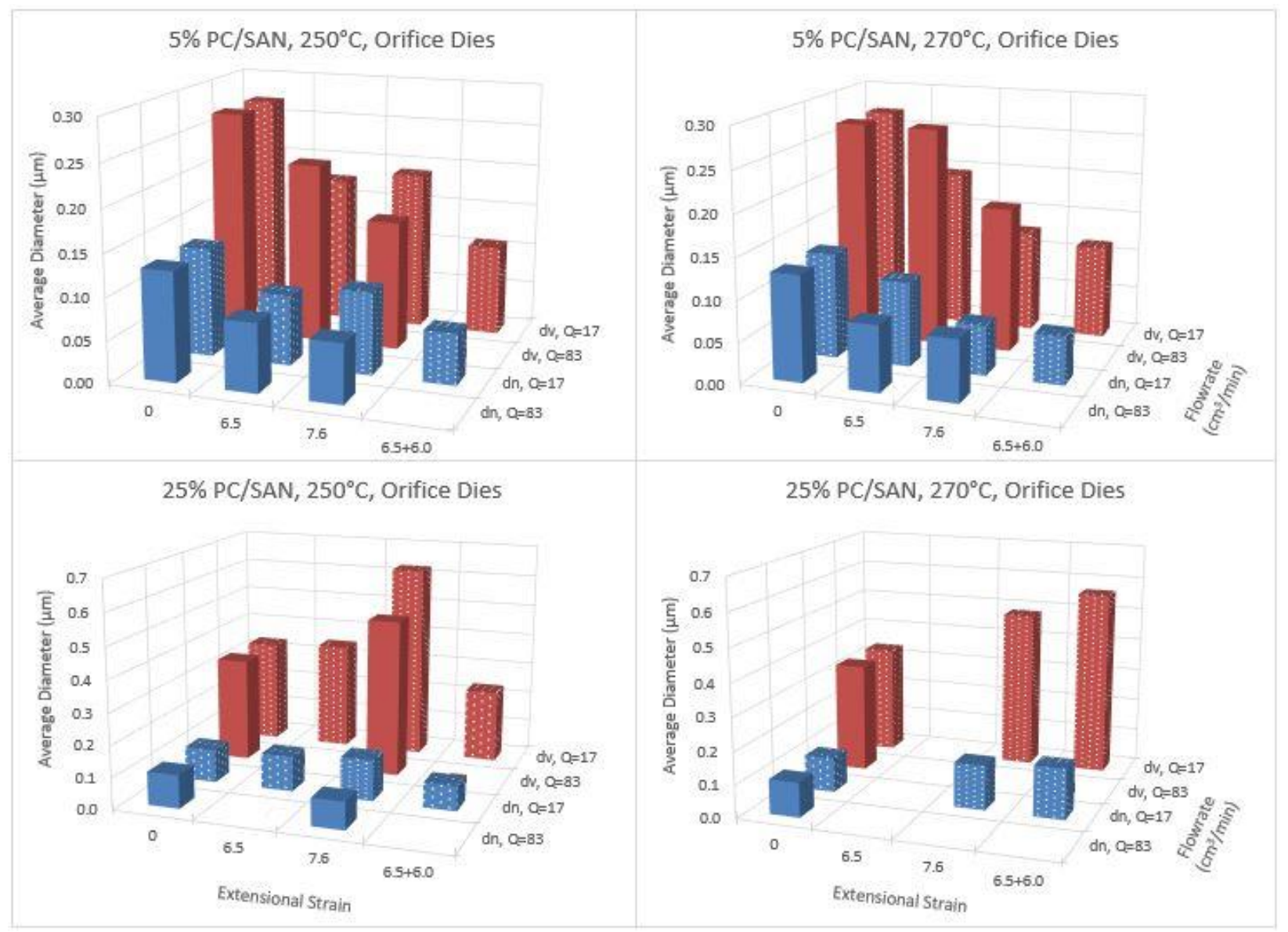

Figure 4-7: PC/SAN phase size with extensional flow through orifice dies. Effects of composition, temperature, extensional strain, and flowrate on $d_{n}$ and $d_{v}$ of PC/SAN through orifice dies. 


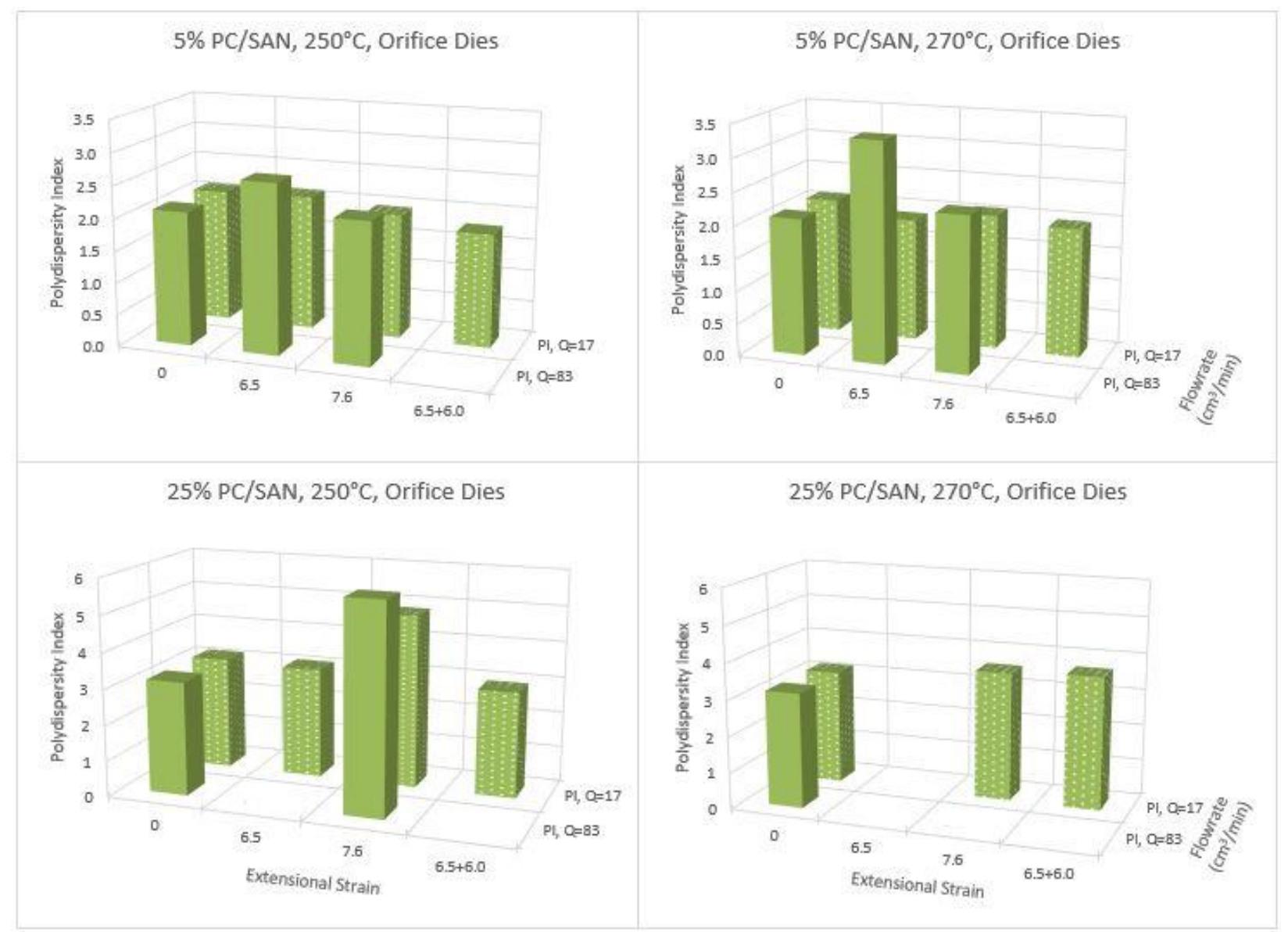

Figure 4-8: PC/SAN phase size distribution with extensional flow through orifice dies. Effects of composition, temperature, extensional strain, and flowrate on the P.I. of PC/SAN through orifice dies. 

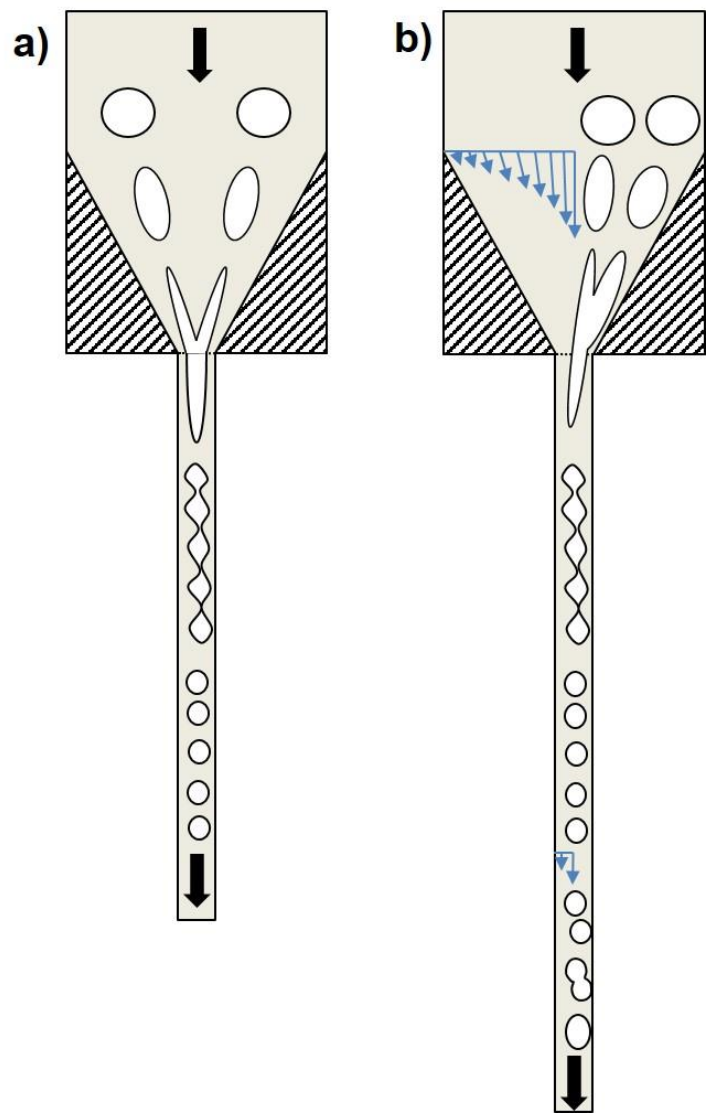

Figure 4-9: Schematics of coalescence during and after extensional flow. Coalescence may occur in the following: a) converging extensional flow fields because fluid streamlines are not parallel and may converge completely like in flow through a converging cone and b) flows where the velocity field is such that drops may overtake and collide with other drops from the side such as that shown in the converging section and during steady shear flow in the downstream tubular flow section.

The effect of the dispersed PC phase concentration on the morphology before and after extensional flow through the stacked orifice dies, $\varepsilon=6.5 \& 6.0$, at $250^{\circ} \mathrm{C}$ and $17 \mathrm{~cm}^{3} / \mathrm{min}$ is given in Figure 4-10. The beginning of each arrow represents the value for the coarse blend, and the end of each arrow represents the value after extensional flow blending. As can be seen, the dispersed phase size increased with increasing concentration, as should be expected due to coalescence which increases with concentration due to an increased number of drop collisions. The coarsening effect of concentration was accentuated in $d_{v}$ compared to $d_{n}$, and therefore the size distribution also increased with increasing concentration. Furthermore, the 
$d_{\mathrm{n}}$ after extensional flow blending through the stacked orifice dies was fairly independent of concentration for dispersed-phase concentrations greater than $1 \mathrm{wt} \%$. This implies that a stable drop size was reached. For $d_{\mathrm{n}}=0.07 \mu \mathrm{m}$, using the apparent shear rate at the wall of the exit of the smallest orifice $=2100 \mathrm{~s}^{-1}$ and an interfacial tension of $0.05 \mathrm{mN} / \mathrm{m}$, the capillary number $\mathrm{Ca}=148$, and the critical capillary number for planar extensional flow at a viscosity ratio of 7.5 is $\mathrm{Ca}_{\text {crit }}=0.21$. This implies that a finer dispersion of PC in SAN is possible yet. Though, compared to typical dispersed polymer-phase sizes of commercially useful blends, this size is already quite fine and likely corresponds to a relatively compatible blend. In practice, dispersed-phase sizes less than about $1 \mu \mathrm{m}$ are desirable, though the specific optimum size depends on the property of interest and the characteristics of the blend constituents.

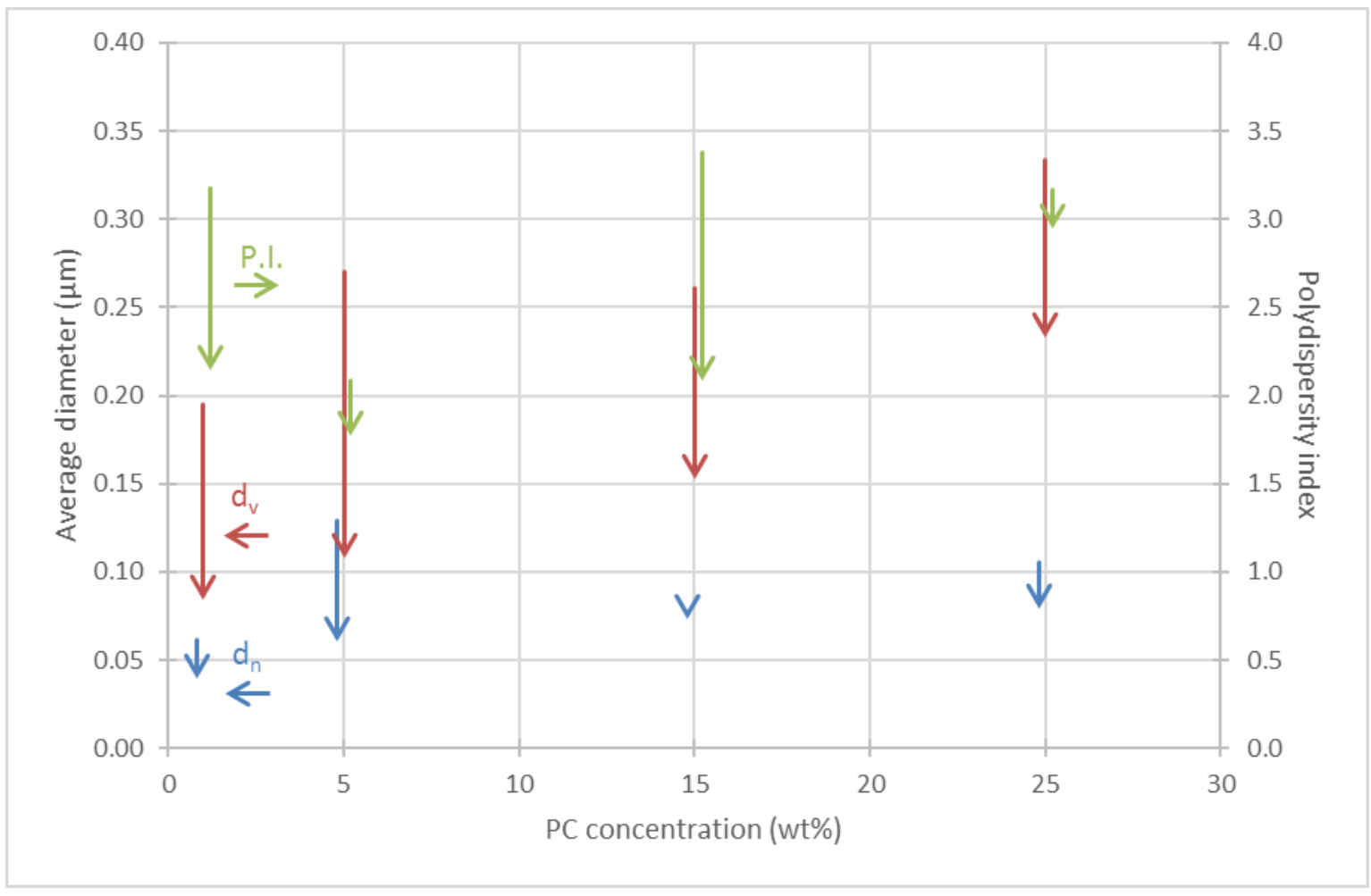

Figure 4-10: Effect of PC/SAN composition on morphology. Change in $d_{\mathrm{n}}, d_{\mathrm{v}}$, and P.I. with extensional flow through both orifice dies (stacked configuration) at $250^{\circ} \mathrm{C}$ at a flowrate of 17 $\mathrm{cm}^{3} / \mathrm{min}$. Beginning of each arrow represents value for the coarse blend, and end of each arrow represents the value after extensional flow. 
In addition to cryo-fracturing the extrudate strands to image the cross section normal to the direction of flow, strands of PC/SAN were also polished from the side in the longitudinal direction. These images are shown in Figure 4-11, and the breakup mechanism of extensional flow is elucidated here. These images show direct observation of elongated threads of the dispersed phase necking due to capillary instabilities and breaking up in a thread breakup mechanism rather than a binary, quasi-steady state breakup.
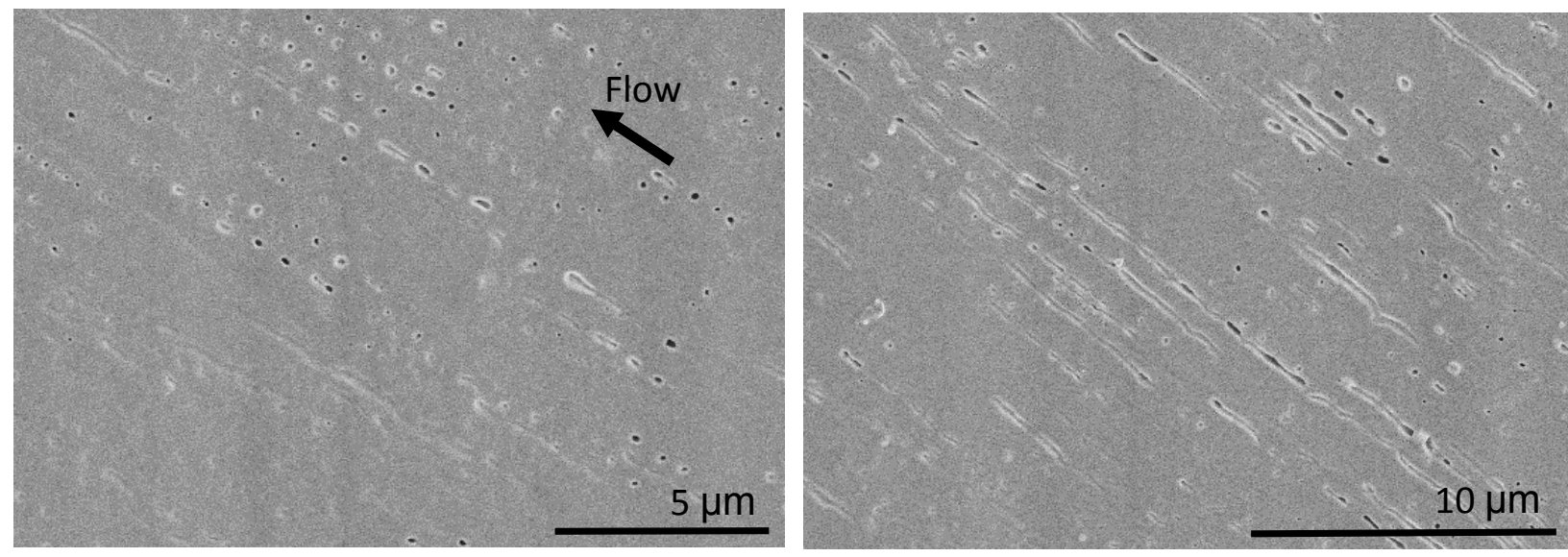

Figure 4-11: SEM of $15 \% \mathrm{PC} / \mathrm{SAN}$ after extensional flow polished in longitudinal direction. Extrudate strands polished in longitudinal direction after extensional flow through stacked orifice dies at $250^{\circ} \mathrm{C}$ and $8 \mathrm{~cm}^{3} / \mathrm{min}$. Left - Image taken near radial edge of strand $(r \rightarrow R)$ and Right - Image taken near radial center of strand $(r \rightarrow 0)$.

Additionally, it is apparent that the dispersed-phase threads break up more readily on the outer edge, at $r \rightarrow R$, compared to those located near the axis of the strand, at $r \rightarrow 0$. This is quantified in Figure 4-12. The effect is likely due to the additional shear contribution to the flow field near the radial edge of the strand, though the relative effects of shear and extensional contributions are likely blend-specific. 


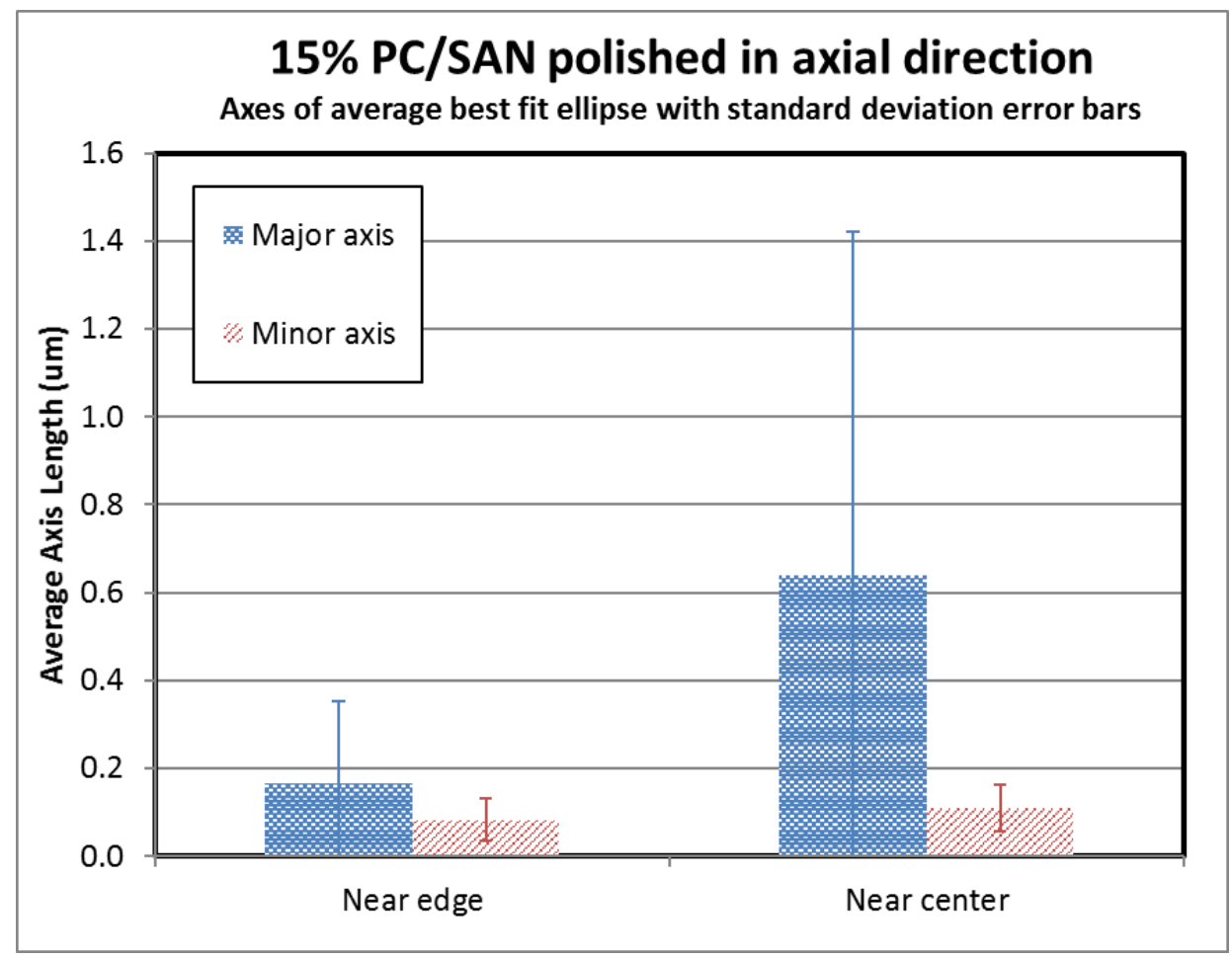

Figure 4-12: Measure of best fit ellipses for polished 15\% PC/SAN after extensional flow. PC drops of SEM images (Figure 4-11) were fitted to ellipses and plotted are the averages for the major and minor axes of the best fit ellipses.

\subsection{PC/SAN through Converging Cone Dies}

PC/SAN blends were also processed through the converging cone dies, described in Section 3.3.2.1. Two dies were fabricated with the same exit diameters as the orifice dies, $0.559 \mathrm{~mm}$ and $0.330 \mathrm{~mm}$, and since the dies had a straight-walled entry tube section with a $D_{\mathrm{i}}=$ $11.7 \mathrm{~mm}$, the dies had total extensional strains of $\varepsilon=6.1$ and 7.1 , respectively. The dies differed from the orifice dies based on the entry profile, which now constricts the flow profile entering the convergence, in contrast to the unrestricted convergence of the orifice dies, where secondary flows consisting of eddies are free to form depending on the material being processed.

The results of extensional flow blending using the converging cone dies on the morphology of PC/SAN are given in Figure 4-13 and compared to the coarse blend and the blends processed through the orifice dies. The $d_{\mathrm{n}}$ was slightly smaller using the converging cone dies, which is expected due to the added shear contribution, but the finest dv attained 
was that after processing through the stacked orifice dies. This implies that an additional stretching episode outweighs the additional shear contribution.

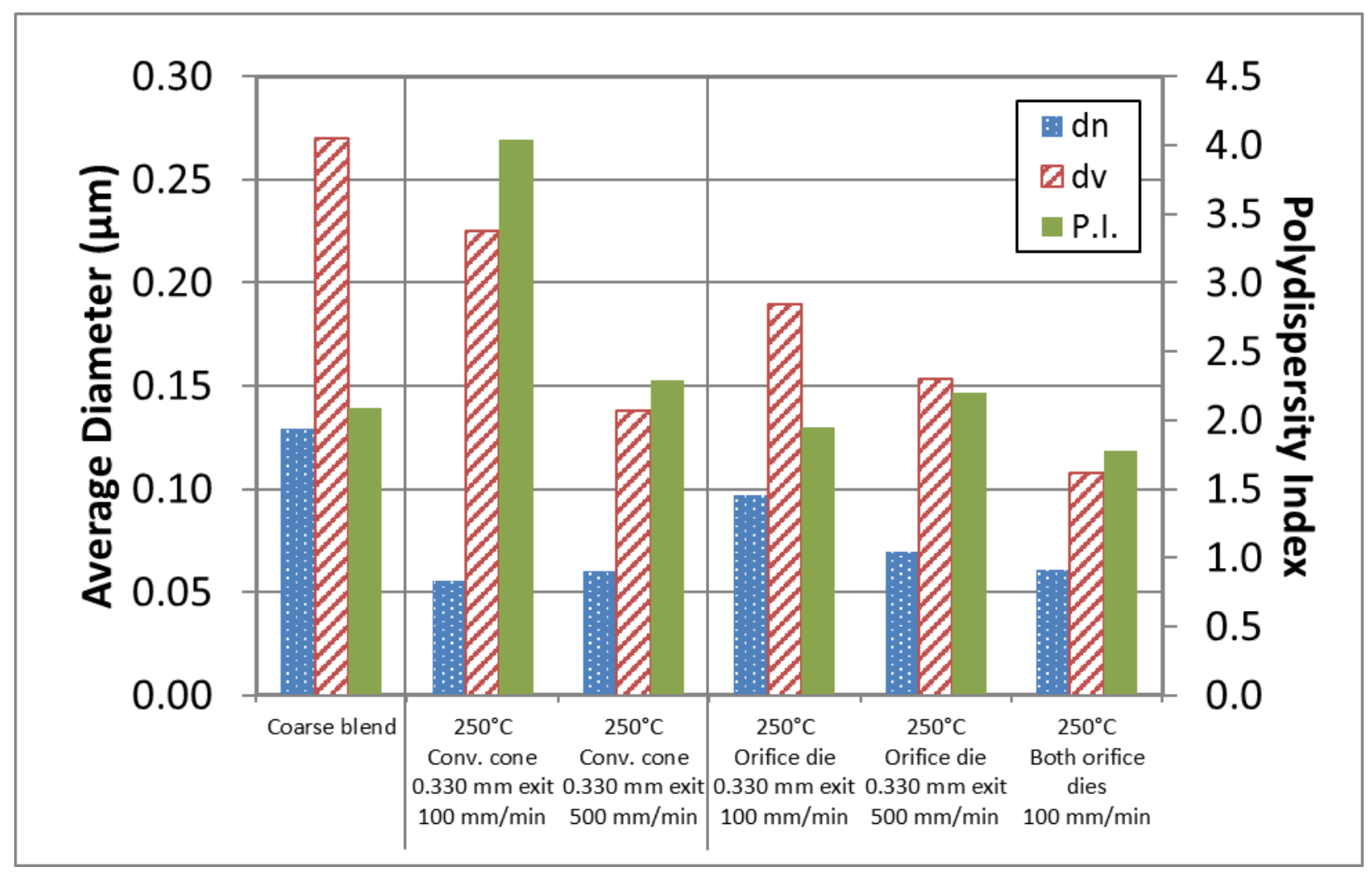

Figure 4-13: Morphology of $5 \% \mathrm{PC} / \mathrm{SAN}$ after extensional flow through converging cone die. Converging cone die with $0.330 \mathrm{~mm}$ exit diameter $(\varepsilon=7.1)$ at 100 and $500 \mathrm{~mm} / \mathrm{min}$ piston speed $\left(Q=17\right.$ and $\left.83 \mathrm{~cm}^{3} / \mathrm{min}\right)$ with comparisons to those through orifice dies.

\subsubsection{Polystyrene (PS)/Linear Low-Density Polyethylene (LLDPE)}

Because the interfacial tension between PC and SAN is low and the phases are compatible, the dispersed phase size was fine even without strong extensional flow mixing. To accentuate the effects and the need for extensional flow, a more incompatible blend was desired. Polystyrene and polyethylene are two of the highest volume commodity thermoplastics with a large selection of grades available on the market. This is also a wellstudied blend system, including some of the foundational literature on extensional rheology [51]. The first PS and PE blend system studied in this work involved two grades already in stock 
in the laboratory. They are PS 500, a general purpose grade from Total Petrochemicals and Petrothene GA 574-000, a linear low-density polyethylene (LLDPE) grade from LyondellBasell, as described in Section 3.1.1.1.

\subsubsection{Rheology of PS/LLDPE}

The viscosity of PS and LLDPE were measured at 180,200 , and $220^{\circ} \mathrm{C}$ with the parallelplate and capillary rheometers. The shear viscosity curves are given in Figure 4-14 along with the viscosity ratio. Since PS is more shear thinning than LLDPE, the viscosity ratio, which is high for low shear rates, falls below the 3.8 viscosity ratio asymptote for pure shear mixing at high shear rates, specifically $\dot{\gamma}>500 \mathrm{~s}^{-1}$. The viscosity of PS is also more sensitive to temperature, so the highest viscosity ratios for the PS/LLDPE blend system are found for the low end of the processing temperature range.

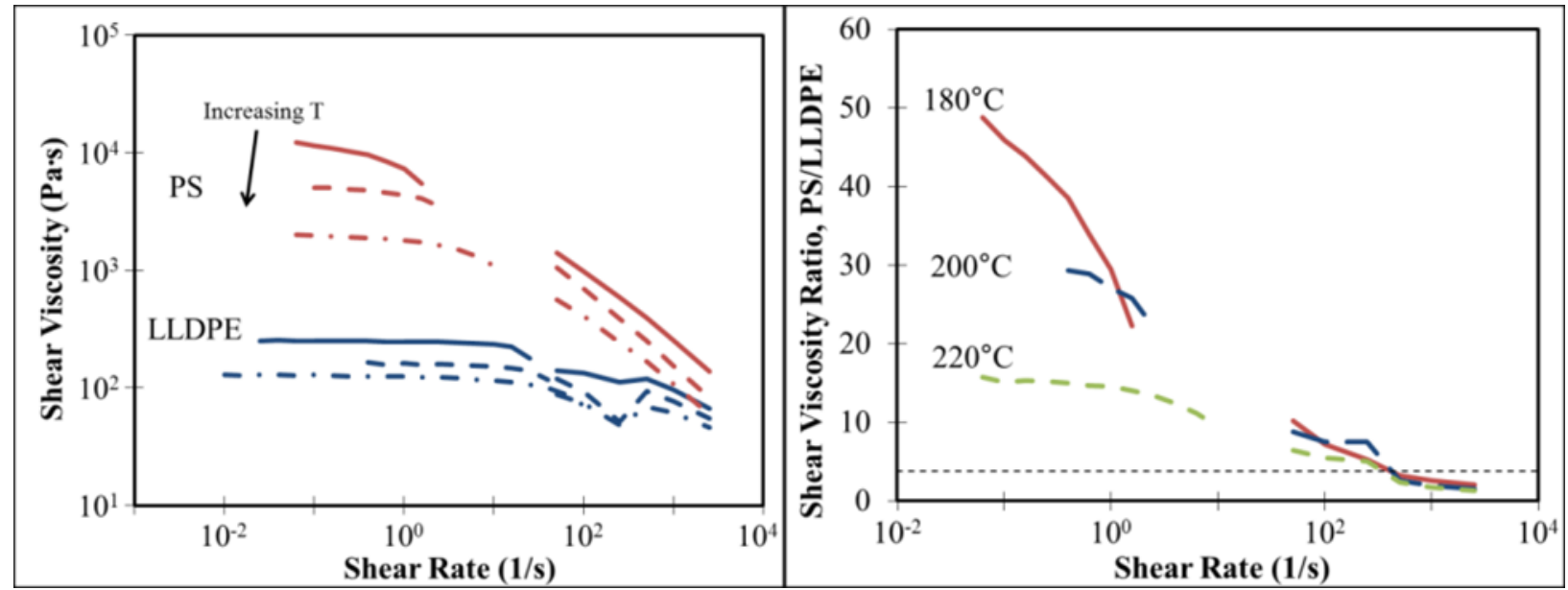

Figure 4-14: PS and LLDPE viscosities and blend viscosity ratio. Shear viscosities of PS and LLDPE at $180^{\circ} \mathrm{C}$ (solid lines), $200^{\circ} \mathrm{C}$ (dashed lines), and $220^{\circ} \mathrm{C}$ (dash-dot lines) measured with parallel-plate rheometer (low $\dot{\gamma}$ ) and capillary rheometer (high $\dot{\gamma}$ ). Viscosity ratio, $p$, exceeds 3.8

$$
\text { for } \dot{\gamma}<500 \mathrm{~s}^{-1} \text {. }
$$

\subsubsection{Interfacial Tension of PS/LLDPE}

As discussed for the PC/SAN blend, surface tension values from the literature for polymers depends greatly on the method of measurement or calculation. The best approach is to measure the surface properties in the laboratory using the same method and exact 
parameters for all of the materials of interest. Because the appropriate equipment was not available for direct measurement of the surface properties, literature values were compiled for polystyrene and polyethylene materials. These data are summarized in Table 4-1, including the data from Souheng $\mathrm{Wu}$, a well-respected researcher in the area [52] and the average values from the other literature referenced, disregarding molecular weight and molecular branching [53-60]. The total surface tension, $\gamma$, is assumed to contain only a disperse part, $\gamma^{d}$, and a polar part, $\gamma^{p}$,

$$
\gamma=\gamma^{\mathrm{d}}+\gamma^{\mathrm{p}},
$$

and the surface polarity, $x^{\mathrm{p}}$, is defined as the relative contribution of the polar part,

$$
x^{\mathrm{p}}=\frac{\gamma^{\mathrm{p}}}{\gamma} .
$$

The dependence of surface tension on temperature for each phase is also given. 
Table 4-1: Surface tension data for PS \& LLDPE

\begin{tabular}{|c|c|c|c|c|c|c|c|c|c|c|c|}
\hline \multirow{2}{*}{$\begin{array}{c}\text { Temperature } \\
\left({ }^{\circ} \mathrm{C}\right)\end{array}$} & \multirow{2}{*}{$\begin{array}{c}\text { Reference } \\
\text { Material }\end{array}$} & \multicolumn{5}{|c|}{ Wu 1982} & \multicolumn{5}{|c|}{ Literature Average (All PS \& PE) } \\
\hline & & $\gamma(\mathrm{mN} / \mathrm{m})$ & $x^{p}(\%)$ & $\gamma^{\mathrm{d}}(\mathrm{mN} / \mathrm{m})$ & $\gamma^{p}(m N / m)$ & $\begin{array}{c}\mathrm{d} v / \mathrm{dT} \\
{[\mathrm{mN} /(\mathrm{m} \cdot \mathrm{K})]}\end{array}$ & $\gamma(\mathrm{mN} / \mathrm{m})$ & $x^{p}(\%)$ & $\gamma^{\mathrm{d}}(\mathrm{mN} / \mathrm{m})$ & $\gamma^{p}(\mathrm{mN} / \mathrm{m})$ & $\begin{array}{c}d \gamma / d T \\
{[\mathrm{mN} /(\mathrm{m} \cdot \mathrm{K})]}\end{array}$ \\
\hline \multirow{2}{*}{20} & PS (High MW) & 40.7 & 16.8 & 33.9 & 6.8 & -0.072 & 39.2 & 5.2 & 37.2 & 2.0 & -0.074 \\
\hline & LLDPE & 33.7 & 0.0 & 33.7 & 0.0 & -0.060 & 34.2 & 2.6 & 33.4 & 0.9 & -0.065 \\
\hline \multirow{2}{*}{180} & PS (High MW) & 29.2 & 16.8 & 24.3 & 4.9 & -0.072 & 27.4 & 5.2 & 26.0 & 1.4 & -0.074 \\
\hline & LLDPE & 24.1 & 0.0 & 24.1 & 0.0 & -0.060 & 23.8 & 2.6 & 23.2 & 0.6 & -0.065 \\
\hline \multirow{2}{*}{200} & PS (High MW) & 27.7 & 16.8 & 23.1 & 4.7 & -0.072 & 26.0 & 5.2 & 24.6 & 1.4 & -0.074 \\
\hline & LLDPE & 22.9 & 0.0 & 22.9 & 0.0 & -0.060 & 22.5 & 2.6 & 22.0 & 0.6 & -0.065 \\
\hline \multirow{2}{*}{220} & PS (High MW) & 26.3 & 16.8 & 21.9 & 4.4 & -0.072 & 24.5 & 5.2 & 23.2 & 1.3 & -0.074 \\
\hline & LLDPE & 21.7 & 0.0 & 21.7 & 0.0 & -0.060 & 21.2 & 2.6 & 20.7 & 0.5 & -0.065 \\
\hline
\end{tabular}


The interfacial tension, $\gamma_{12}$, at temperature may be calculated from $\gamma, \gamma^{\mathrm{d}}$, and $\gamma^{\mathrm{p}}$ of each polymer constituent. The two most common equations for this calculation are the harmonic mean and geometric mean equations:

$$
\begin{gathered}
\gamma_{12, \text { harmonic }}=\gamma_{1}+\gamma_{2}-4\left(\frac{\gamma_{1}^{\mathrm{d}} \gamma_{2}^{\mathrm{d}}}{\gamma_{1}^{\mathrm{d}}+\gamma_{2}^{\mathrm{d}}}+\frac{\gamma_{1}^{\mathrm{p}} \gamma_{2}^{\mathrm{p}}}{\gamma_{1}^{\mathrm{p}}+\gamma_{2}^{\mathrm{p}}}\right) \\
\gamma_{12, \text { geometric }}=\gamma_{1}+\gamma_{2}-2\left(\sqrt{\gamma_{1}^{\mathrm{d}} \gamma_{2}^{\mathrm{d}}}+\sqrt{\gamma_{1}^{\mathrm{p}} \gamma_{2}^{\mathrm{p}}}\right) .
\end{gathered}
$$

The harmonic mean equation is commonly used for relatively low surface energy and nonpolar materials like polymers, and the geometric mean equation is more common for higher surface energy materials. The surface tension properties and interfacial tensions at various temperatures are given in Table 4-2, including data from [52] and the average from the rest of the literature. As can be seen, both the harmonic mean and geometric mean equations yield relatively low interfacial tension values for the PS/LLDPE blend, compared to the literature value of $1.4 \mathrm{mN} / \mathrm{m}$ for the PC/SAN blend and compared to the average values from the literature for PS/PE. These calculated interfacial tensions seem questionable based on the typical results from the literature and those from this chapter, in which PS/PE blends are typically quite incompatible and yield relatively coarse morphologies. The interfacial tension between PS and LLDPE is discussed further in Section 4.3.3.1.

\begin{tabular}{|c|c|c|c|c|c|c|}
\hline \multirow[b]{2}{*}{ Temperature $\left({ }^{\circ} \mathrm{C}\right)$} & \multirow{2}{*}{$\begin{array}{c}\text { Reference } \\
\text { Blend }\end{array}$} & \multicolumn{2}{|c|}{ Wu 1982} & \multicolumn{3}{|c|}{ Literature Average (All PS \& PE) } \\
\hline & & $\begin{array}{c}\gamma_{12, \text { harmonic }} \\
\text { mean } \\
(\mathrm{mN} / \mathrm{m})\end{array}$ & $\begin{array}{c}\gamma_{12, \text { geometric }} \\
\text { mean } \\
(\mathrm{mN} / \mathrm{m})\end{array}$ & $\begin{array}{c}\gamma_{12, \text { harmonic }} \\
\text { mean } \\
(\mathrm{mN} / \mathrm{m})\end{array}$ & $\begin{array}{c}\gamma_{12, \text { geometric }} \\
\text { mean } \\
(\mathrm{mN} / \mathrm{m})\end{array}$ & $\begin{array}{c}\gamma_{12, \text { Literature }} \\
\text { average } \\
(\mathrm{mN} / \mathrm{m})\end{array}$ \\
\hline 20 & \multirow{4}{*}{ PS/LLDPE } & 6.84 & 6.84 & 0.67 & 0.35 & 8.30 \\
\hline 180 & & 4.90 & 4.90 & 0.49 & 0.25 & 5.45 \\
\hline 200 & & 4.66 & 4.66 & 0.46 & 0.24 & 5.48 \\
\hline 220 & & 4.42 & 4.42 & 0.44 & 0.23 & 5.47 \\
\hline
\end{tabular}

Table 4-2: Interfacial tension between PS/LLDPE

\subsubsection{PS/LLDPE Coarse Blend Mixing Time}

Similar to the coarse blend mixing time analysis that was done on PC/SAN, a corresponding analysis was performed for the PS/LLDPE blend system. 15\% PS/LLDPE was 
mixed for up to $20 \mathrm{~min}$ at $200^{\circ} \mathrm{C}$ and rotor speed of $80 \mathrm{rpm}$. The samples were extracted from the inner area between the rotors. The results are given in Figure 4-15 along with comparisons to an extruded coarse blend and two batches of coarse blends prepared in the internal mixer at $200^{\circ} \mathrm{C}, 80 \mathrm{rpm}, 6 \mathrm{~min}$. Again, as was observed for PC/SAN, $d_{\mathrm{n}}$ increases after $1 \mathrm{~min}$ then remains fairly consistent. From $1-5 \mathrm{~min}, d_{\mathrm{v}}$ is fairly constant, then $d_{\mathrm{v}}$ decreases at $10 \mathrm{~min}$ and $20 \mathrm{~min}$. This could imply that further mixing is occurring for this blend system up to those times, or thermal aging could be decreasing the viscosity of the dispersed PS phase, the viscosity of which is more temperature-sensitive than the matrix LLDPE phase. This would effectively decrease the viscosity ratio and allow for the formation of finer morphologies. The mixing time analysis results are compared to the morphologies of the extruded coarse blend and two batches of coarse blends prepared using internal mixer processing parameters of $200^{\circ} \mathrm{C}, 80 \mathrm{rpm}, 6 \mathrm{~min}$. Unsurprisingly, the extruded coarse blend produces $d_{\mathrm{v}}$ comparable to the smallest sizes returned from the internal mixer. The TSE screws are designed for compounding and include kneading blocks which should be more effective at blending the high-viscosity ratio blend than the roller rotors of the internal mixer. Though $d_{\mathrm{n}}$ of the extruded coarse blend was large, perhaps again due to flow-induced coalescence, the extruder produced the narrowest size distribution, as evidenced by the lowest P.I. value. Finally, it can be seen that the morphologies of the two batches of coarse blends each mixed for $6 \mathrm{~min}$ were very consistent with the morphology in this analysis at $5 \mathrm{~min}$. This consistency and reproducibility confirmed that $6 \mathrm{~min}$ is sufficient mixing time in the internal mixer to produce a relatively stable morphology for PS/LLDPE blends. 


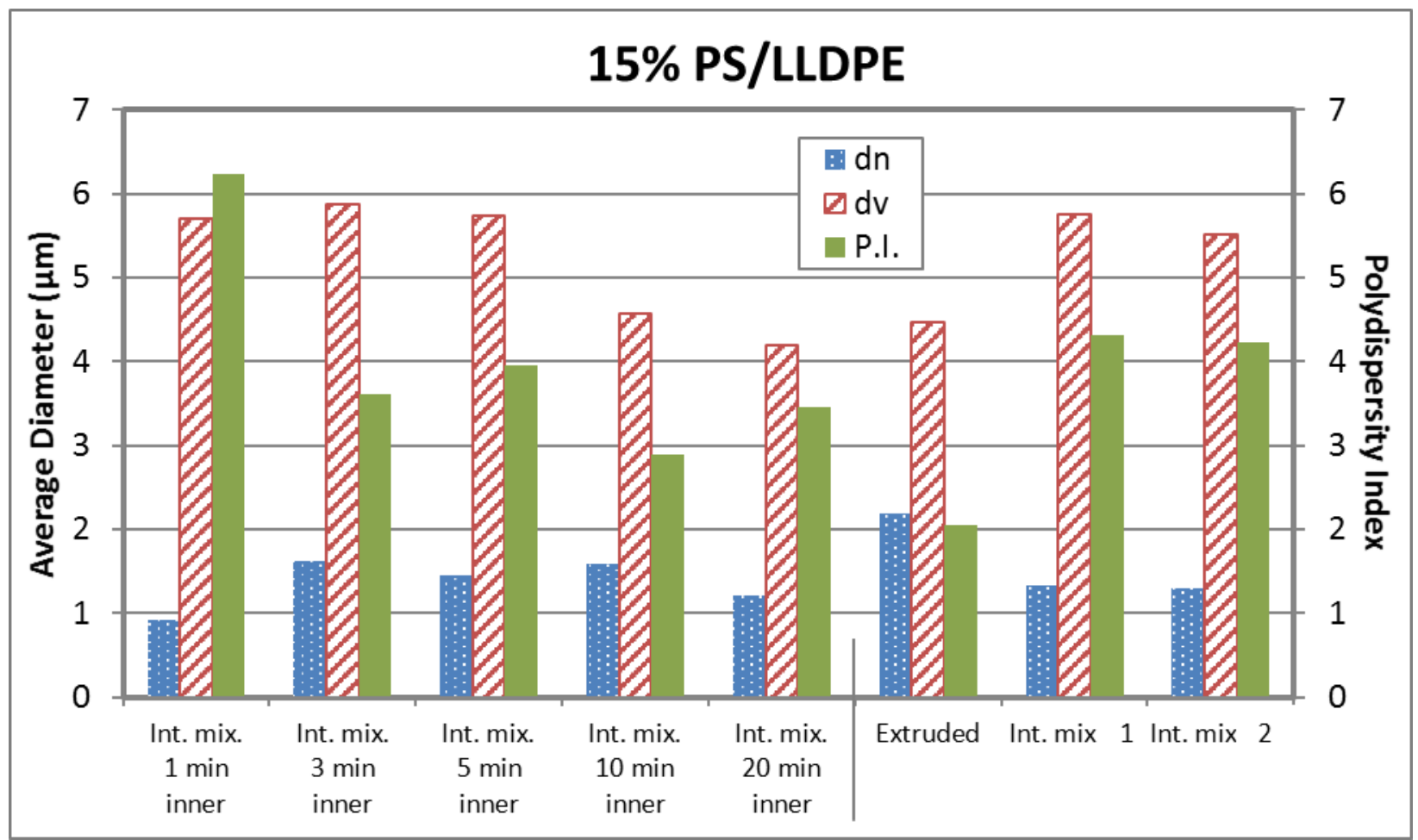

Figure 4-15: Effect of mixing time on morphology of $15 \%$ PS/LLDPE. Mixing at $200^{\circ} \mathrm{C}$ with roller rotor speed of $80 \mathrm{rpm}$. Comparisons with extruded blend and two batches of coarse blends prepared with 6 min mixing time.

\subsubsection{Extensional Flow Blending of PS/LLDPE through Hemisphere-in-Cone Dies}

The PS/LLDPE preliminary blend system was processed through several extensional flow cells (EFCs), including the hemisphere-in-cone EFC which is described in Section 3.3.2.4. The EFC consists of an insert with a hemispherical end that nests within the converging section of the converging cone die with $0.559 \mathrm{~mm}$ exit diameter. This produces two stretching episodes for a single pass through the die, and the first stretching episode contains contributions of planar extensional flow which is more efficient for drop deformation and breakup than uniaxial extensional flow. Furthermore, the extensional strain of the first stretching episode may be controlled by adding or removing spacers between the insert and the shoulder upon which it rests.

Figure 4-16 shows several different ways that the morphology of a blend may be characterized, specifically for 5\% PS/LLDPE before and after extensional flow blending through the hemisphere-in-cone die with a 0.002 in $(0.059 \mathrm{~mm})$ spacer at two different flow rates. 
Inspecting only the $d_{n}, d_{v}$, and P.I. descriptors, it appears that the extensional flow blending using this geometry and parameters had little positive effects on the morphology, though $d_{\mathrm{n}}$ slightly decreased, $d_{v}$ slightly increased, and therefore the spread, P.I., increased. Inspecting the histograms, however, a decrease in the frequency of dispersed-phase drops in the midrange size is observed. The overall range of drop size is fairly consistent before and after extensional flow blending, but fewer drops of mid-range size are found and more very small drops are found after extensional flow blending. It is likely that the dispersed phase morphology after extensional flow is a mixture of quasi-spherical drops and frozen-in elongated threads. Threads are expected to have very small diameters compared to quasi-spherical drops both before and after drop deformation and breakup. Since the images are all of the cross section of the extruded strand, the elongated thread domains appear as circular domains. The representative SEM images corroborate the observations in that overall range of size is constant, with more very small circular domains observed after extensional flow blending. 


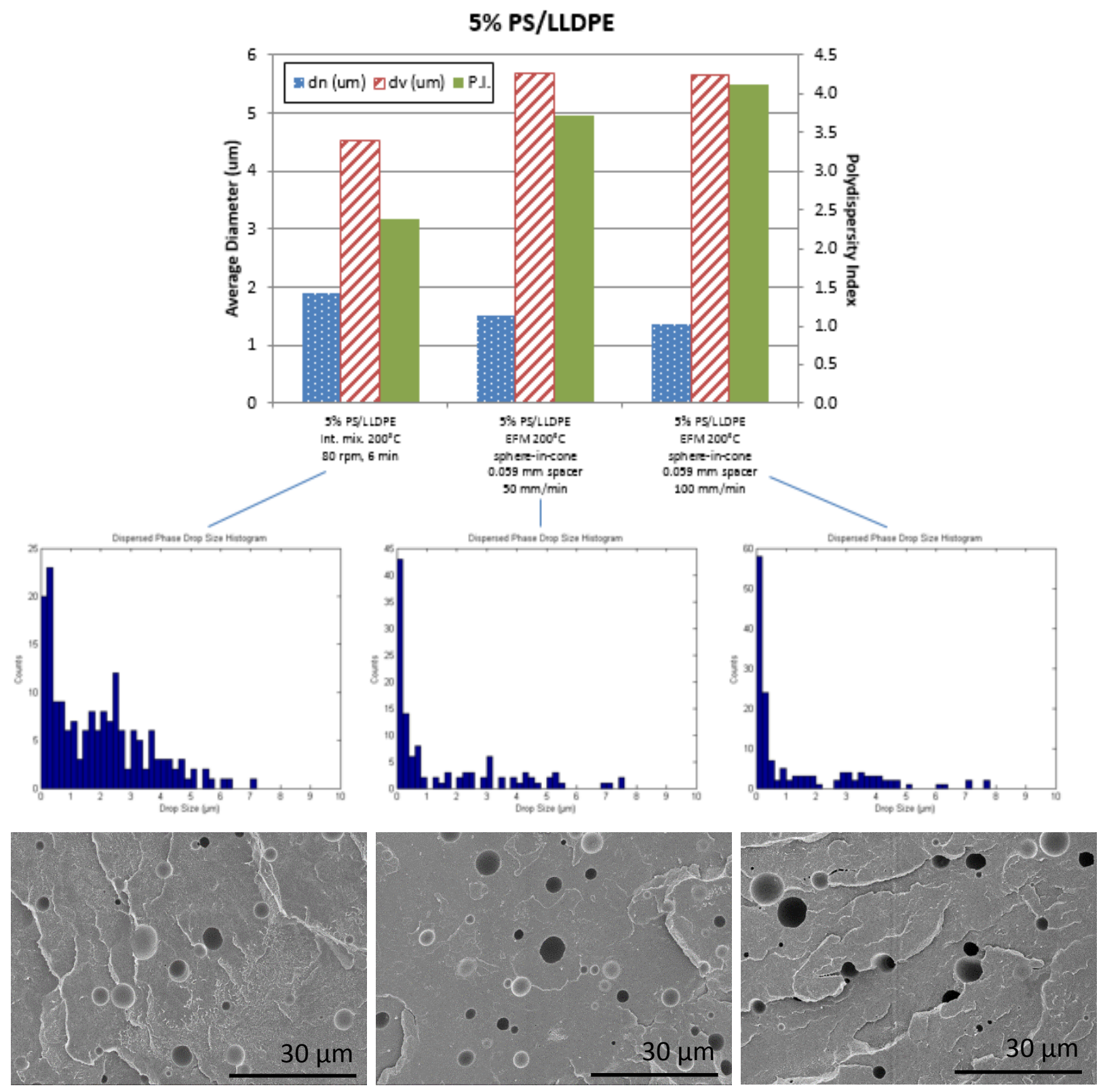

Figure 4-16: Morphological effects of 5\% PS/LLDPE using hemisphere-in-cone die. Effects of flow rate on morphology of 5\% PS/LLDPE through hemisphere-in-cone die with 0.002 in $(0.052$ $\mathrm{mm}$ ) spacer at $200^{\circ} \mathrm{C}$. Top $-d_{\mathrm{n}}, d_{\mathrm{v}}$, and P.I. descriptors, middle - discrete histograms of the dispersed-phase drop size distribution, and bottom - representative SEM images.

The effect of flow rate and extensional strain through the first convergence are also given in Figure 4-17 for extensional flow blending of 15\% PS/LLDPE through the hemisphere-incone die. Two different spacer settings were investigated with two different flow rates through 
each. The coarse blend for 15\% PS/LLDPE was prepared using a twin-screw extruder as well, rather than the internal mixer for most of the blends of this work.

The size of the dispersed phase was slightly higher using 15\% PS/LLDPE compared to 5\% PS/LLDPE, as is expected due to coalescence; though, extensional flow blending of this blend through the hemisphere-in-cone die had a similar effect as it did on the 5\% PS/LLDPE blend. The $d_{\mathrm{n}}$ slightly decreased or stayed the same, while $d_{\mathrm{v}}$ increased. A larger gap, or spacer, setting yielded slightly larger $d_{v}$ than the smallest spacer of 0.002 in $(0.052 \mathrm{~mm})$. The latter effect is expected because the extensional strain through the first stretching episode, between the hemisphere insert and the converging cone wall, is smaller for larger spacers or gaps. The effects of flow rate and the overall effect that extensional flow blending through the EFC increased $d_{v}$ are not intuitive. The morphology descriptors $d_{n}, d_{v}$, and P.I. may not fully resolve the effects of this blending operation, and this is an issue to take into account in the analysis using the main blend of interest, HDPE/PS.

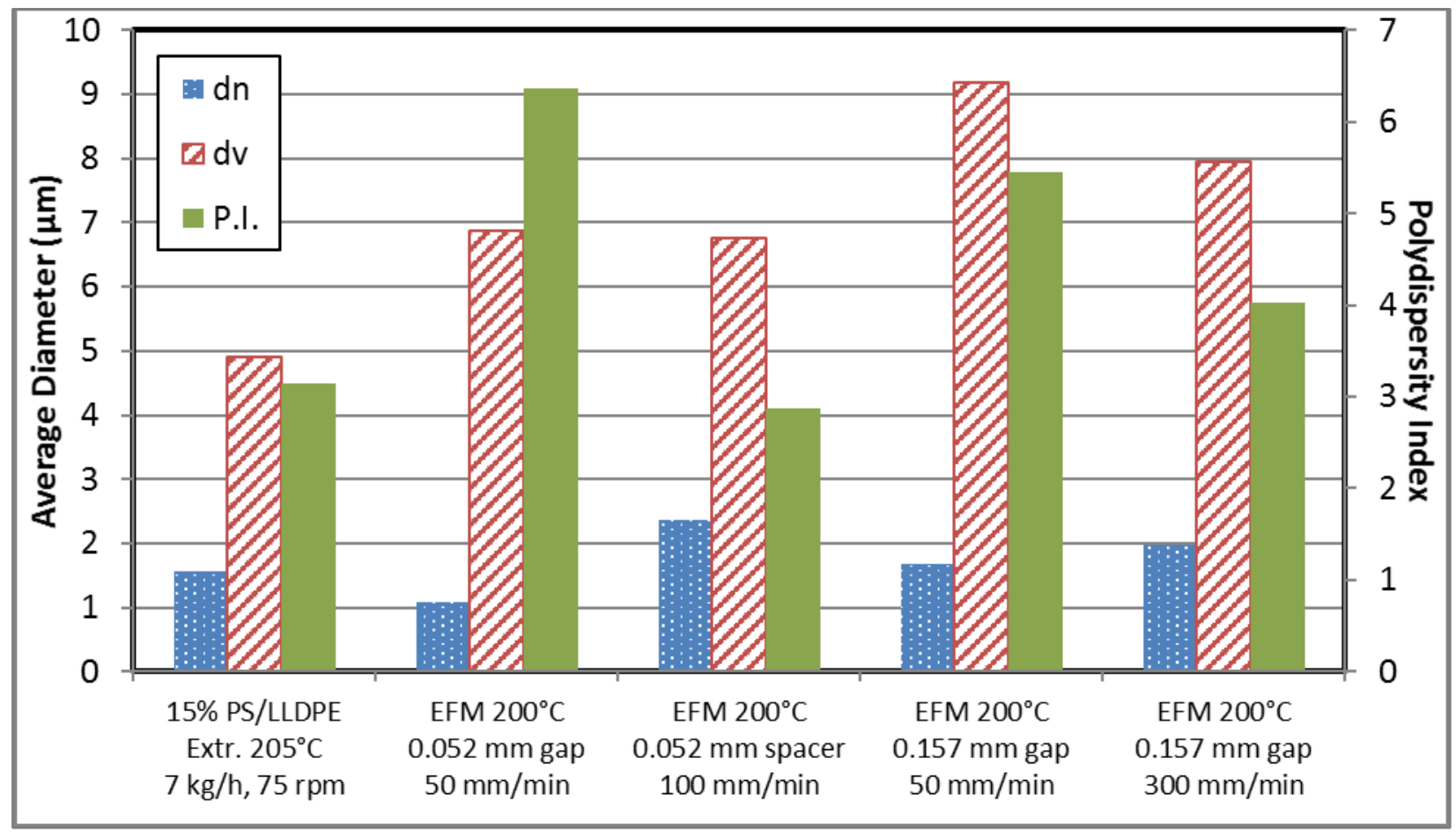

Figure 4-17: Morphological effects of 15\% PS/LLDPE using hemisphere-in-cone die. Effects of flow rate and gap setting of the first stretching episode on the morphology of $15 \%$ PS/LLDPE through hemisphere-in-cone die at $200^{\circ} \mathrm{C}$. 


\subsubsection{High-Density Polyethylene (HDPE)/Polystyrene (PS)}

Other blend systems investigated for this work include polycarbonate/polylactic acid (PLA). This blend system was studied in detail in this laboratory by Finniss in pursuit of PLAbased blends suitable for durable applications [32], and PLA has also been compounded with nanoclay fillers to produce nanocomposites for moisture-barrier packaging applications [61]. The phase contrast between the phases was not as distinct as other blend systems, however, and further exploration into PE and PS grades was done to find a suitable blend system with reasonably high interfacial tension and high viscosity ratio across all processing conditions. Several grades of PS and PE were obtained from various industrial suppliers and characterized based on their rheology, which will be discussed in the following section. The final blend system chosen for detailed investigation was a blend of high-density polyethylene (HDPE) dispersed in PS.

\subsubsection{Rheology of HDPE/PS}

To fully exploit the advantage of extensional flow in blending, it was desired to ensure that the blend viscosity ratio was greater than the asymptote for pure shear flow, that is maintain $p>3.8$, for the entire shear rate range the blend would encounter in processing. This amplifies the need for extensional flows to enable drop stretching and breakup and effectively disperse a high-viscosity phase into a low-viscosity matrix. For the PS/LLDPE blend, as shown in Figure 4-14, viscosity ratio drops below the asymptote for pure shear mixing between 250 and $500 \mathrm{~s}^{-1}$ for all temperatures because PS is more shear thinning than LLDPE. Viscosity ratio is inversely proportional to temperature for this blend system, so the highest viscosity ratios are achieved at the lowest appropriate processing temperatures.

As shown in Figure 4-18, this is within the range of typical shear rates present in processing in single-screw and twin-screw extruders. The shear rates in the converging cone dies and hemisphere-in-cone dies were estimated using Equations (4-5) and (4-6). Equation (4-5) calculates the apparent shear rate for a Newtonian liquid flowing through a circular crosssection tube of radius $R$ at volumetric flowrate $Q$, and Equation (4-6) is the apparent shear rate through a rectangular slit of width $W$ and height $H$ modified for the gap between the rounded 
edge (hemisphere) insert and the converging cone wall with $R$ at that place in the converging cone and $G$ being the gap distance.

$$
\begin{gathered}
\dot{\gamma}_{\text {app }}=-\frac{4 Q}{\pi R^{3}} \\
\dot{\gamma}_{\text {app }}=-\frac{6 Q}{W H^{2}}=-\frac{6 Q}{\pi R^{2} G^{2}}
\end{gathered}
$$

The ranges for these estimates show that the shear rates expected in the converging cone and sphere-in-cone dies are greater than the maximum shear rate where the viscosity ratio of PS/LLDPE is greater than 3.8. For this reason, other grades of polyethylene and polystyrene were sought after to ensure the viscosity ratio was large for as broad a range of shear rates as possible.

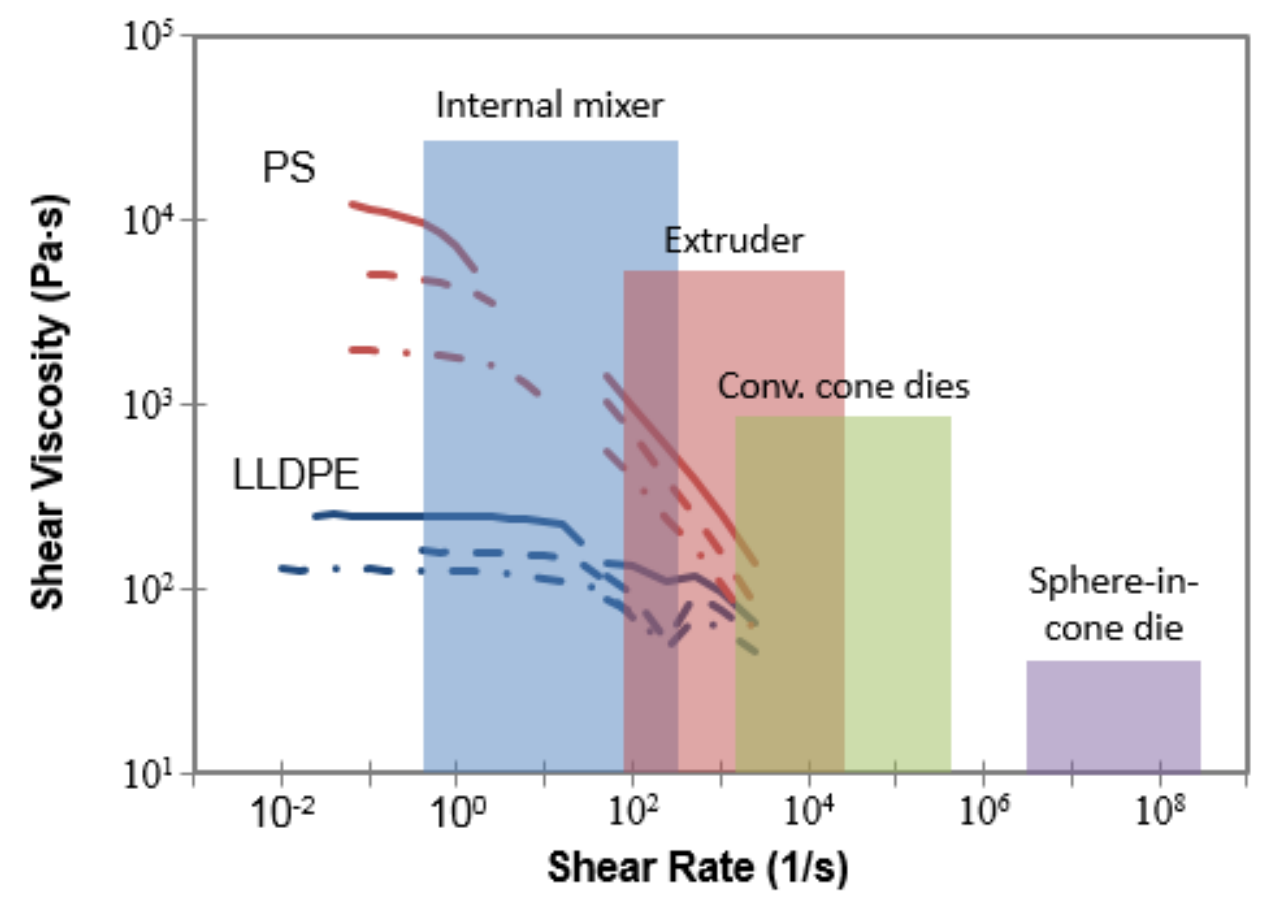

Figure 4-18: Viscosity curves for PS and LLDPE with typical process shear rate ranges

Several grades of polystyrene and polyethylene were acquired and their viscosities measured to identify blends with the largest viscosity ratios. The reported melt flow index and measured shear viscosity curve for each grade of PS are given in Table 4-3 and Figure 4-19, and those for each grade of PE are given in Table 4-4 and Figure 4-20. The zero shear viscosities of 
select grades of PE and PS as measured using the parallel plate rheometer are also given in Table 4-5.

Table 4-3: Melt flow indices of PS grades

\begin{tabular}{|l|l|c|}
\hline $\begin{array}{l}\text { Grade name } \\
\text { Styron }{ }^{\circledR} \text { 685D (PS }\end{array}$ & Manufacturer & Melt Flow Index (g/10 min)* \\
\hline PS 500 & Americas Styrenics & 1.5 \\
\hline PS XU36305 & $\begin{array}{l}\text { Ameral Petrochemicals Styrenics (developmental } \\
\text { grade) }\end{array}$ & 23 \\
\hline
\end{tabular}

*Measured at $200^{\circ} \mathrm{C}$ using $5 \mathrm{~kg}$ weight.

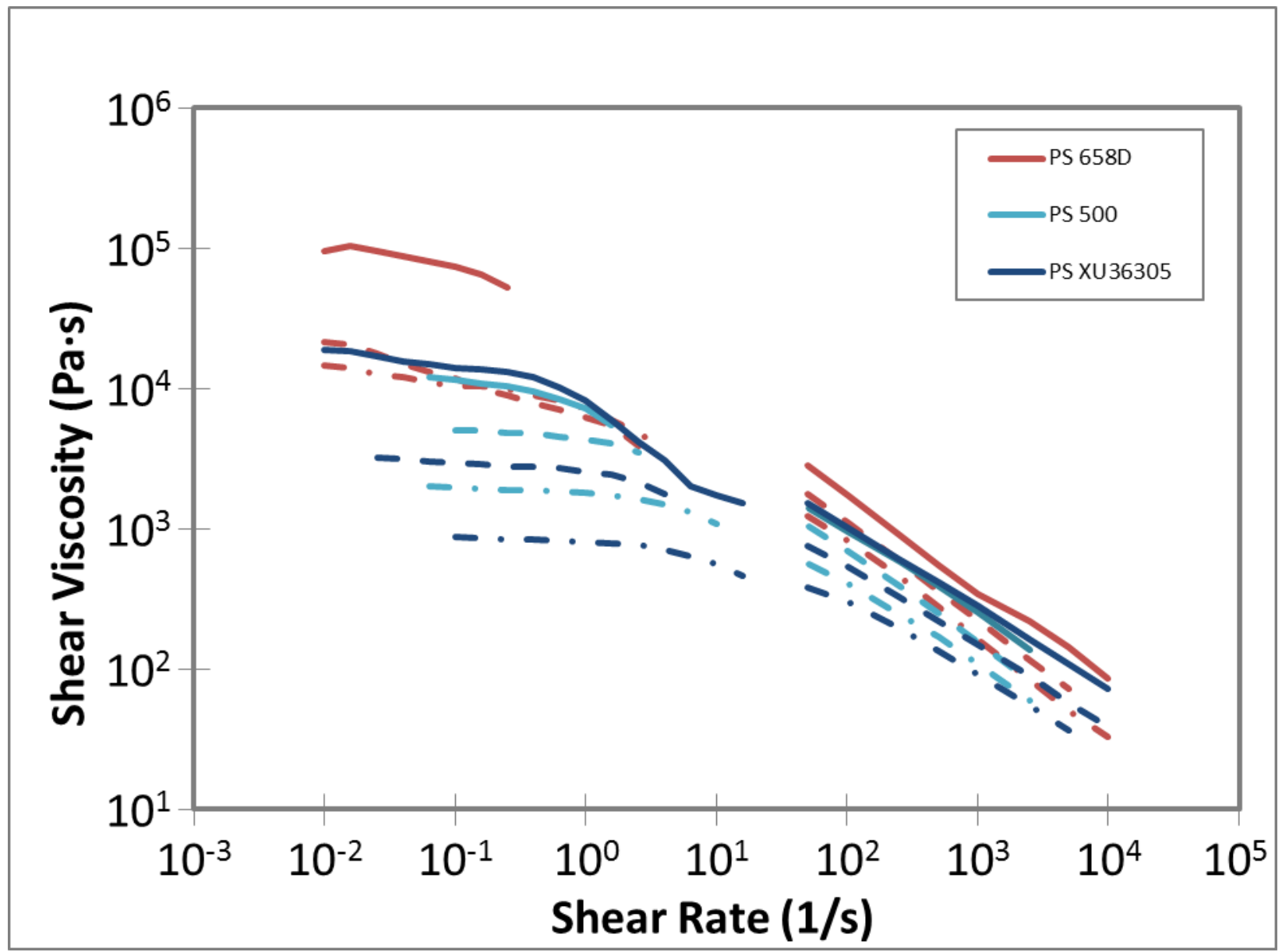

Figure 4-19: Shear viscosity curves of PS grades. Low shear rates measured with RMS 800 parallel plate rheometer; high shear rates measured with capillary rheometer. Solid lines, $180^{\circ} \mathrm{C}$; dashed lines, $200^{\circ} \mathrm{C}$, dash-dot lines, $220^{\circ} \mathrm{C}$. 
Table 4-4: Melt flow indices of PE grades

\begin{tabular}{|c|c|c|}
\hline Grade name & Manufacturer & Melt Flow Index $(\mathrm{g} / 10 \mathrm{~min})^{*}$ \\
\hline $\begin{array}{l}\text { NOVAPOL }^{\circledR} \text { HB-W355-A (HDPE } \\
\text { HBW355A) }\end{array}$ & Nova Chemicals & $3.0 * *$ \\
\hline Marlex $^{\circledR} 1412$ (LDPE 1412) & Chevron Phillips & 35.0 \\
\hline Marlex ${ }^{\circledR} 9035$ (HDPE 9035) & Chevron Phillips & 40.0 \\
\hline $\begin{array}{l}\text { Petrothene }{ }^{\circledR} \text { GA 574-000 } \\
\text { (LLDPE) }\end{array}$ & LyondellBasell & 50 \\
\hline SCLAIR $^{\circledR} 2114$ (LLDPE 2114) & Nova Chemicals & 52 \\
\hline SCLAIR $^{\circledR} 2316$ (MDPE 2316) & Nova Chemicals & 72 \\
\hline
\end{tabular}

*Measured at $190^{\circ} \mathrm{C}$ with a $2.16 \mathrm{~kg}$ weight unless otherwise noted.

$* *$ High Load Melt Index (HLMI), measured at $190^{\circ} \mathrm{C}$ with a $21.6 \mathrm{~kg}$ weight.

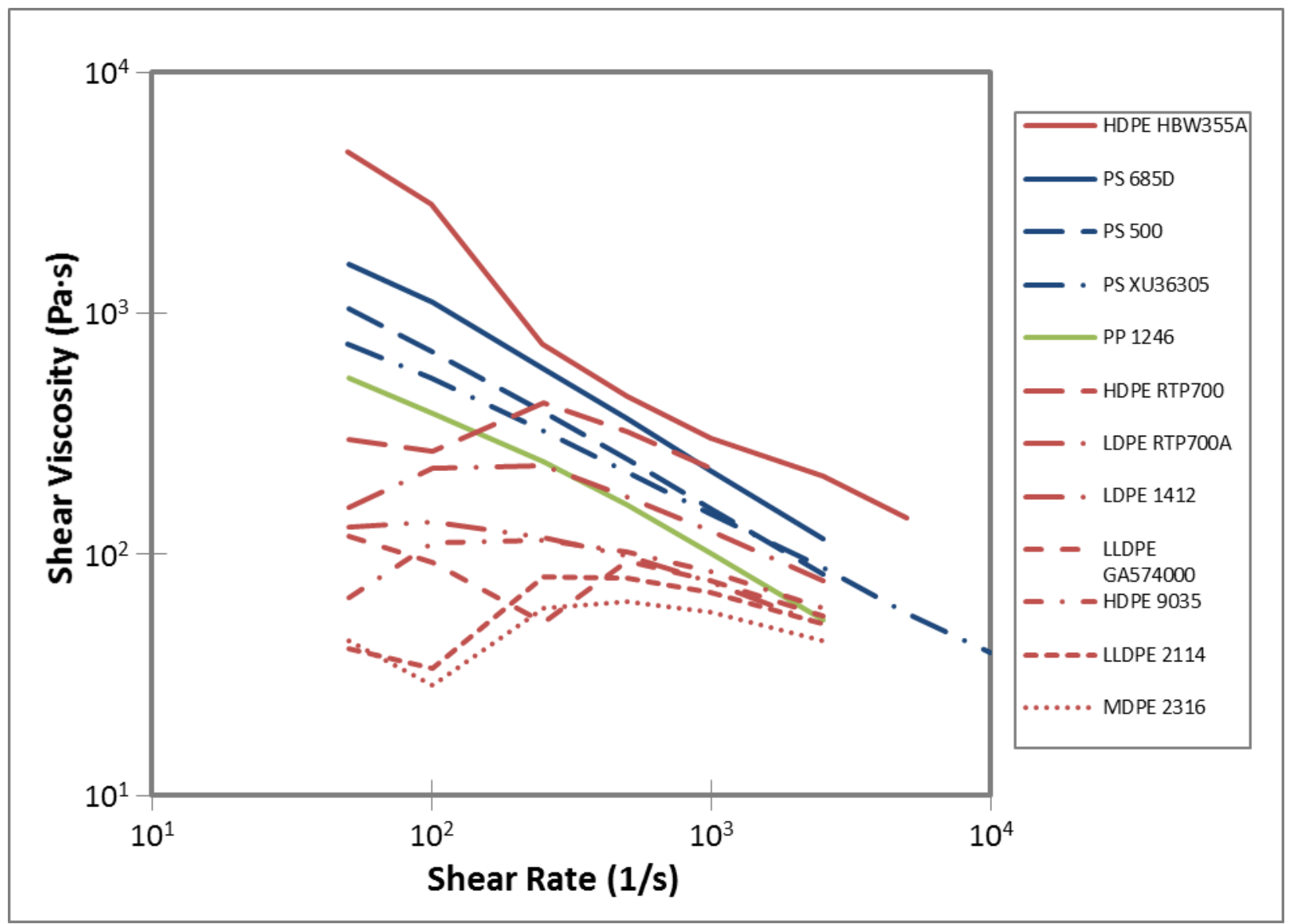

Figure 4-20: Shear viscosity curves of PE grades. Measured with capillary rheometer at $200^{\circ} \mathrm{C}$. 
Table 4-5: Measured zero-shear viscosity for PE and PS grades. Measured with parallel-plate rheometer.

\begin{tabular}{|c|c|c|c|c|c|}
\hline Material & $\begin{array}{l}\text { Temperature } \\
\left({ }^{\circ} \mathrm{C}\right)\end{array}$ & $\begin{array}{l}\text { Zero-Shear } \\
\text { Viscosity } \\
\text { (Pa.s) }\end{array}$ & Material & $\begin{array}{l}\text { Temperature } \\
\left({ }^{\circ} \mathrm{C}\right)\end{array}$ & $\begin{array}{l}\text { Zero-Shear } \\
\text { Viscosity } \\
\text { (Pa.s) }\end{array}$ \\
\hline \multirow{3}{*}{$\begin{array}{l}\text { HDPE } \\
\text { HBW355A }\end{array}$} & 180 & 148,590 & \multirow[t]{3}{*}{ PS 685D } & 180 & 98,208 \\
\hline & 200 & 113,641 & & 200 & 21,493 \\
\hline & 220 & 104,719 & & 220 & 14,520 \\
\hline \multirow{3}{*}{$\begin{array}{l}\text { LLDPE } \\
\text { GA574000 }\end{array}$} & 180 & 249 & \multirow[t]{3}{*}{ PS 500} & 180 & 12,142 \\
\hline & 200 & 160 & & 200 & 5,009 \\
\hline & 220 & 127 & & 220 & 1,999 \\
\hline \multirow[t]{3}{*}{ LLDPE 2114} & 180 & 240 & \multirow[t]{3}{*}{ PS XU36305 } & 180 & 19,083 \\
\hline & 200 & 170 & & 200 & 3,245 \\
\hline & 220 & 116 & & 220 & 884 \\
\hline \multirow[t]{3}{*}{ MDPE 2316} & 180 & 185 & & & \\
\hline & 200 & 125 & & & \\
\hline & 220 & 88 & & & \\
\hline
\end{tabular}

For blends of PS dispersed in PE, the highest viscosity PS is PS 685D, and the lowest viscosity PE is MDPE 2316. The viscosity curves and viscosity ratio for that blend system are shown in Figure 4-21. As observed for the previously used PS/LLDPE system, PS is more shear thinning than PE, so the viscosity ratio of the blend drops below the asymptote for pure shear mixing at high shear rates. Also, again viscosity ratio is inversely proportional to temperature. The viscosity ratio for PS 685D/MDPE 2316 is as high as 450 at $180^{\circ} \mathrm{C}$ and does not drop below the pure shear mixing asymptote until the shear rate is higher than $2,500 \mathrm{~s}^{-1}$. This shear rate is again lower than some of those expected in the converging cone and sphere-in-cone dies, but the range of shear rates where the viscosity ratio is high is extended compared to the PS/LLDPE system. In the end, the PS/MDPE blend was not studied in detail in this work because the following blend system, HDPE/PS, proved to yield a wider domain of shear rates over which the viscosity ratio was greater than 3.8 . 

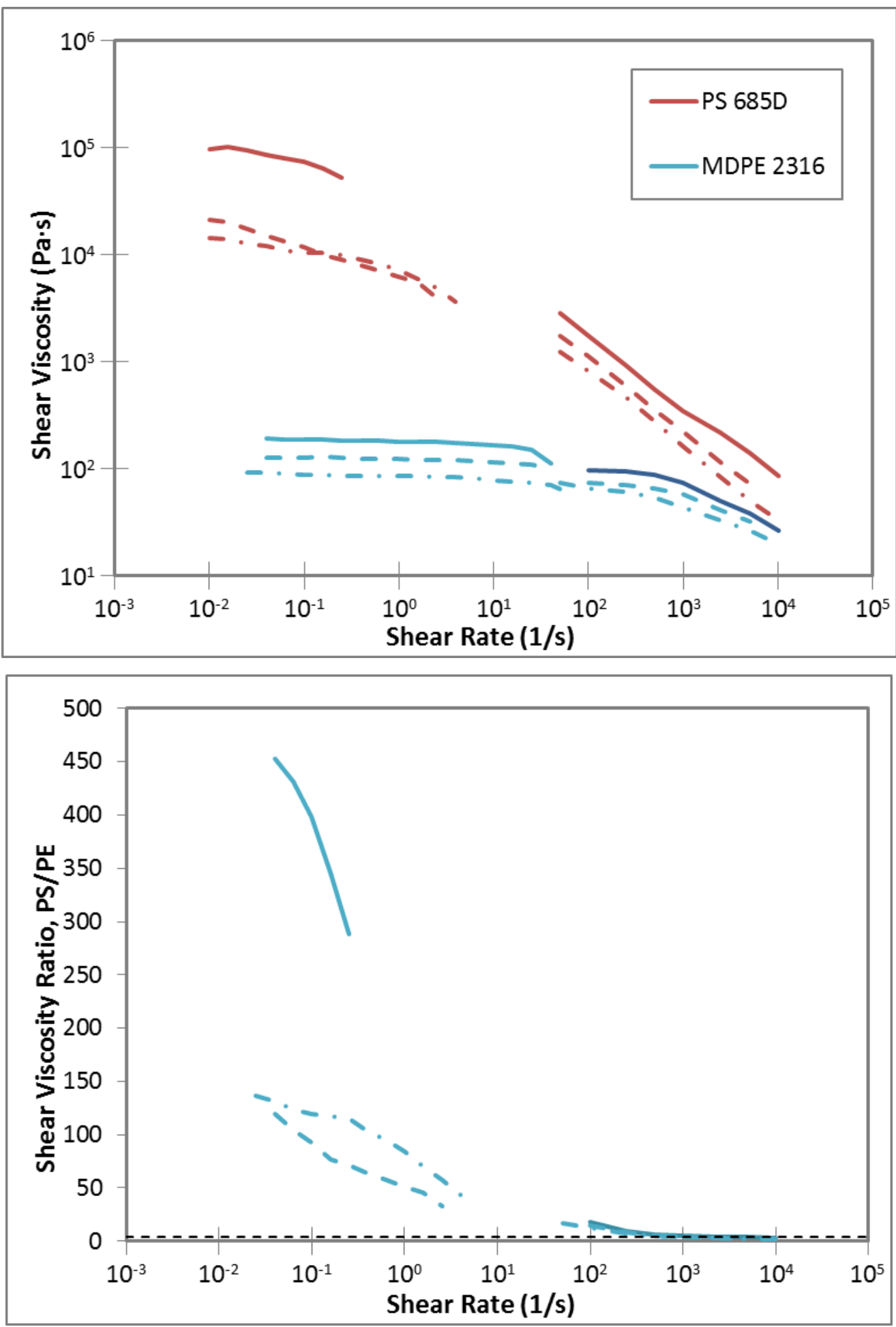

Figure 4-21: Shear viscosity curves for PS 685D and MDPE 2316. Top - Shear viscosity curves for PS $685 \mathrm{D}$ and MDPE 2316 . Solid lines, $180^{\circ} \mathrm{C}$; dashed lines, $200^{\circ} \mathrm{C}$, dash-dot lines, $220^{\circ} \mathrm{C}$. Bottom - Viscosity ratio for PS/PE systems at three different temperatures along with asymptote for pure shear mixing at viscosity ratio $=3.8$. 
For blends of PE dispersed in PS, HDPE HB-W355-A is the highest viscosity grade of PE, and PS XU36305 is the lowest viscosity PS. The shear viscosity of these grades was measured more extensively using both the parallel-plate rheometer and the capillary rheometer. For the parallel-plate rheometer, shear rate sweeps and dynamic frequency sweeps at various temperatures, along with the Cox-Merz Rule and time-temperature superposition (TTS), were used, and shear rate sweeps on the capillary rheometer were also used to measure the shear viscosity at as broad a shear rate domain as possible. The shear viscosity vs. shear rate curves at temperatures throughout the processing temperature window are shown in Figure 4-22. Since the viscosity of PS is more temperature-sensitive than that of HDPE, the maximum operating temperature of $220^{\circ} \mathrm{C}$ yielded the largest viscosity ratio. The shear viscosity curves at $220^{\circ} \mathrm{C}$ are also compared to those of the unfilled 5\% HDPE/PS and $4 \%$ one step-mixed nanosilica-filled 5\% HDPE/PS blends in Figure 4-23. Blending and mixing nanoparticles did not significantly affect the shear viscosity response of the material.

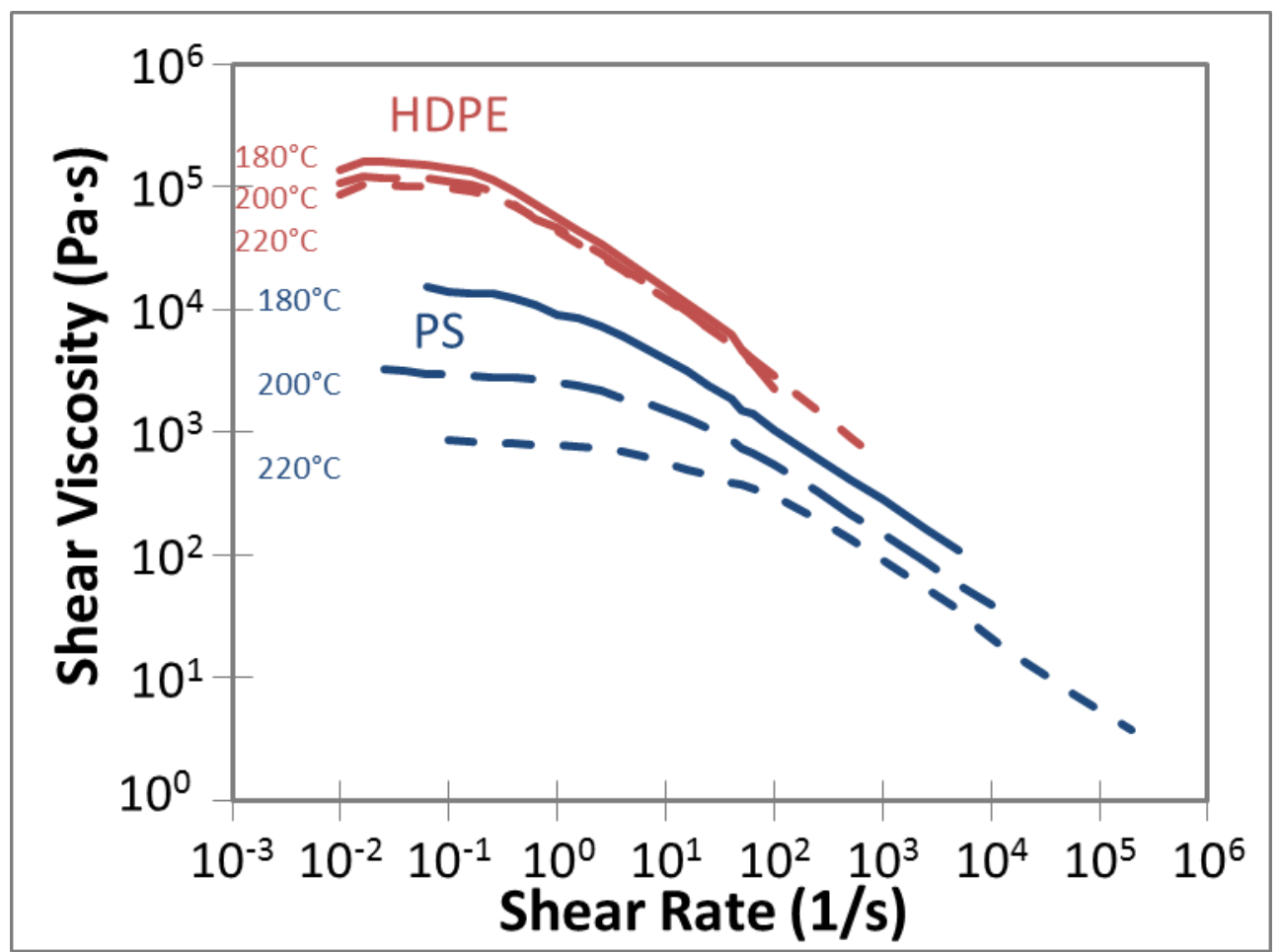

Figure 4-22: Shear viscosity curves for HDPE HBW355A and PS XU36305. Measured with parallel-plate rheometer using shear rate sweeps, the Cox-Merz Rule, and time-temperature superposition (TTS) and with a capillary rheometer at 180,200 , and $220^{\circ} \mathrm{C}$. 


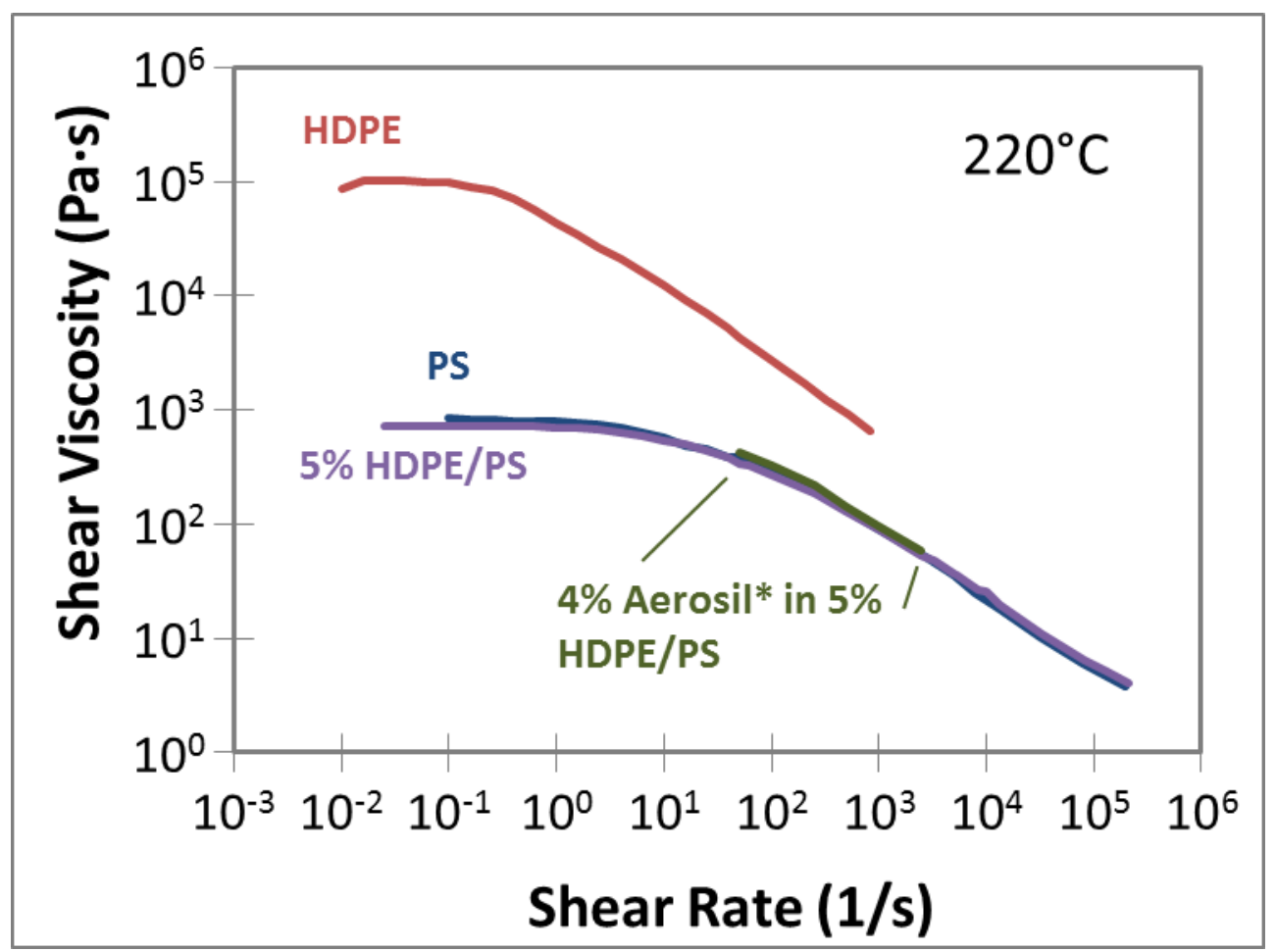

Figure 4-23: Shear viscosity of HDPE and PS compared to that of blends at $220^{\circ} \mathrm{C}$. Comparison of the shear viscosity vs. shear rate curves at $220^{\circ} \mathrm{C}$ between HDPE, PS, $5 \%$ HDPE/PS, and $4 \%$ nanosilica in $5 \%$ HDPE/PS. For the nano-filled blend rheology, the data from one step-mixed blends using Aerosil 200 (hydrophilic) and Aerosil R104 (hydrophobic) are combined.

The viscosity ratio vs. shear rate is plotted in Figure 4-24 for the measured rheology data as well as that which was extrapolated by fitting to the power-law model. As can be seen, the viscosity ratio is greater than the 3.8 asymptote for pure shear mixing in the Grace curve, Figure $2-4$, for all shear rates expected in the processing of this work at a melt temperature of $220^{\circ} \mathrm{C}$. For shear rates greater than about $2 \times 10^{6} \mathrm{~s}^{-1}$, however, the viscosity ratio is predicted to drop below 3.8 because HDPE has a lower power-law index, $n$, than does PS. The power-law viscosity model describes the shear-thinning behavior that polymeric liquids exhibit at the shear rates of most processing. It is defined in Equation (2-5). The shear behavior of HDPE and PS at $220^{\circ} \mathrm{C}$ were fit to a constitutive equation given by the union of the zero-shear Newtonian plateau, as given in Equation (2-4), and power-law shear-thinning behavior. The fitted parameters are given in Table 4-6, and the fitted curves are plotted on the left of Figure 4-25. 
The fitted constitutive equation for the PS matrix was also used to calculate the shear stress across the shear rate domain, and this was used to plot the shear viscosity ratio vs. shear stress on the right in Figure 4-25. This is useful for polymer blends because shear stress is usually assumed to be conserved across the interface between the dispersed-phase and matrix-phase polymers. Alternatively, interfacial tension contributes to the relationship between the shear stress of the dispersed phase and that of the matrix phase, and active interfaces further complicate the boundary condition.

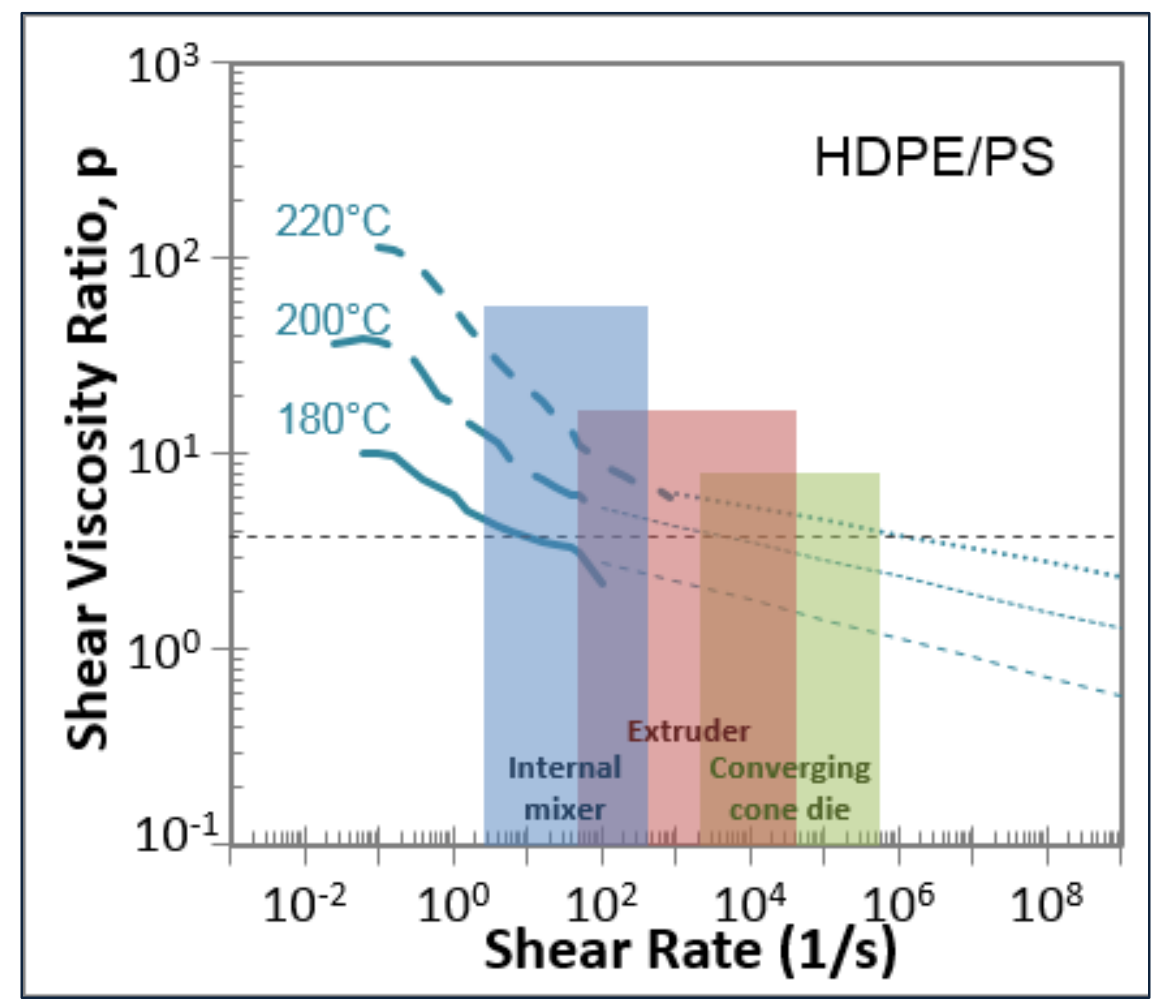

Figure 4-24: Shear viscosity ratio of HDPE/PS Thick curves - measured; thin curves extrapolation using the power-law model. The viscosity ratio for HDPE/PS at $220^{\circ} \mathrm{C}$ is greater than the 3.8 asymptote for pure shear mixing from the Grace plot for all shear rates anticipated in the processing of this work. 
Table 4-6: Rheological fit parameters for HDPE \& PS

\begin{tabular}{|l|c|c|}
\hline $\begin{array}{l}\text { Zero-shear (Newtonian } \\
\text { plateau) viscosity (Pa·s) }\end{array}$ & HDPE (Grade HB-W355-A) & PS (Grade XU36305) \\
\hline Transition shear rate (1/s) & 0.6 & 27 \\
\hline Power-law consistency & 65,800 & 6,430 \\
metric, K (Pa·s) & 0.314 & 0.381 \\
\hline Power-law index, $\mathbf{n}$ & & \\
\hline
\end{tabular}
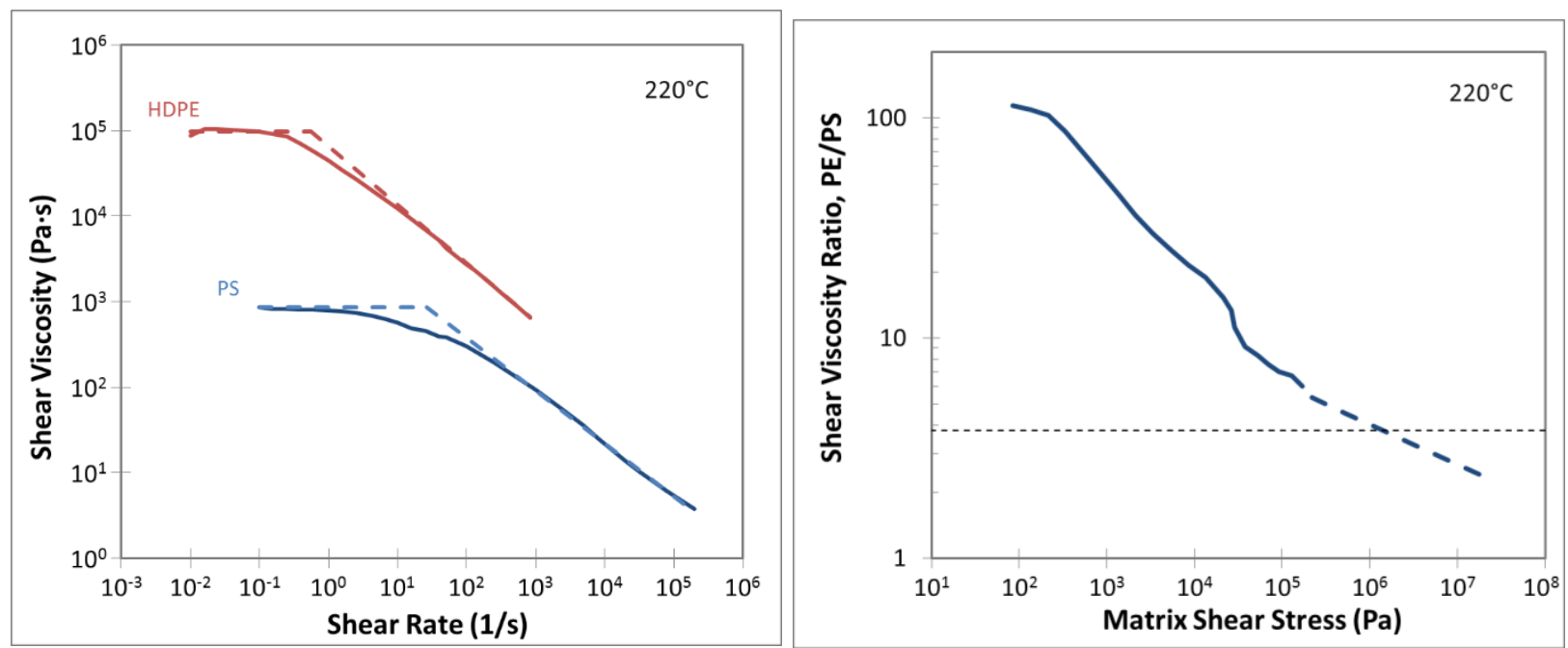

Figure 4-25: Rheological fits and viscosity ratio vs. shear stress. Left - HDPE and PS shear viscosity vs. shear rate fitted using zero-shear Newtonian plateau and power-law shear-thinning behavior; Right - Shear viscosity ratio of HDPE/PS vs. matrix shear stress, as calculated using the rheological fits. Solid curves are measured, and dashed curves are calculated using rheological fits.

The dynamic rheology of the final blend system used was investigated using a Rheometrics RMS800 rotational rheometer. A parallel plate fixture with $25 \mathrm{~mm}$ diameter plates was used. Initial strain sweeps at $0.01 \mathrm{~Hz}$ frequency found that $2-12 \%$ strain was generally required to ensure testing was done in the linear viscoelastic region. Dynamic frequency sweeps then elucidate the rheological terms of interest. The results of several 
dynamic frequency sweeps for both HDPE HB-W355-A and PS XU36305 at $220^{\circ} \mathrm{C}$ are given in Figure 4-26 and Figure 4-27, respectively. The dynamic rheology curves for the 5\% HDPE/PS blend are also given in Figure 4-28. Shown for each are the elastic storage modulus $G^{\prime}$, viscous loss modulus $G^{\prime \prime}$, complex viscosity $\eta^{*}$, and tangent of phase angle $\tan \delta$.

The crossover point at which $G^{\prime}$ overtakes $G^{\prime \prime}$ and the material's flow response changes from viscous to rubbery elastic is observed for HDPE. This is given by the crossover frequency $\omega_{\mathrm{c}}$ for which $G^{\prime}=G^{\prime \prime}=G_{\mathrm{c}}$. This is also denoted where $\tan \delta=1$. The crossover point was estimated for PS and the 5\% HDPE/PS blend by fitting $\log G^{\prime}$ vs. $\log \omega$ and $\log G^{\prime \prime}$ vs. $\log \omega$ to quadratic functions and extrapolating to higher frequencies. These are listed in Table 4-7 along with the calculated characteristic relaxation time $\lambda_{r}$, which can be estimated by the inverse of $\omega_{c}:$

$$
\lambda_{\mathrm{r}}=\frac{1}{\omega_{\mathrm{c}}} .
$$

The use of Equation (4-7) to measure the relaxation time, $\lambda_{r}$, is an empirical method that is not fully supported by robust theory. Theoretical calculations, assuming a constitutive rheological behavior of the polymer phase such as the upper-convected Maxwell model, are commonly used in the literature and relate $\lambda_{\mathrm{r}}$ to the storage modulus $G^{\prime}$ and the shear viscosity $\eta_{\mathrm{s}}$ at a given frequency or shear rate. These calculations were considered in the work as well and yielded similar results to the crossover point calculation presented.

The relaxation time of HDPE at $2.7 \mathrm{~s}$ is significant. This can be on the same order as or greater than the processing time in the extensional flow cells (EFCs). This would imply that the Deborah number De,

$$
\mathrm{De}=\frac{\lambda_{\mathrm{r}}}{t_{\mathrm{c}}},
$$

where $t_{\mathrm{c}}$ is the characteristic process time which in this case would be the approximate residence time in the EFC, exceeds unity. This in turn implies that the elastic nature of the HDPE dispersed phase is significant during this processing. Furthermore, the Weissenberg number Wi,

$$
\mathrm{Wi}=\lambda_{\mathrm{r}} \dot{\varepsilon}
$$


may become very large, implying that the elastic nature of the HDPE significantly delays the response of the dispersed phase to the extensional flow stresses. The dispersed phase deformation will surely not reach its equilibrium state, and transient and elastic effects will be important in these flows.

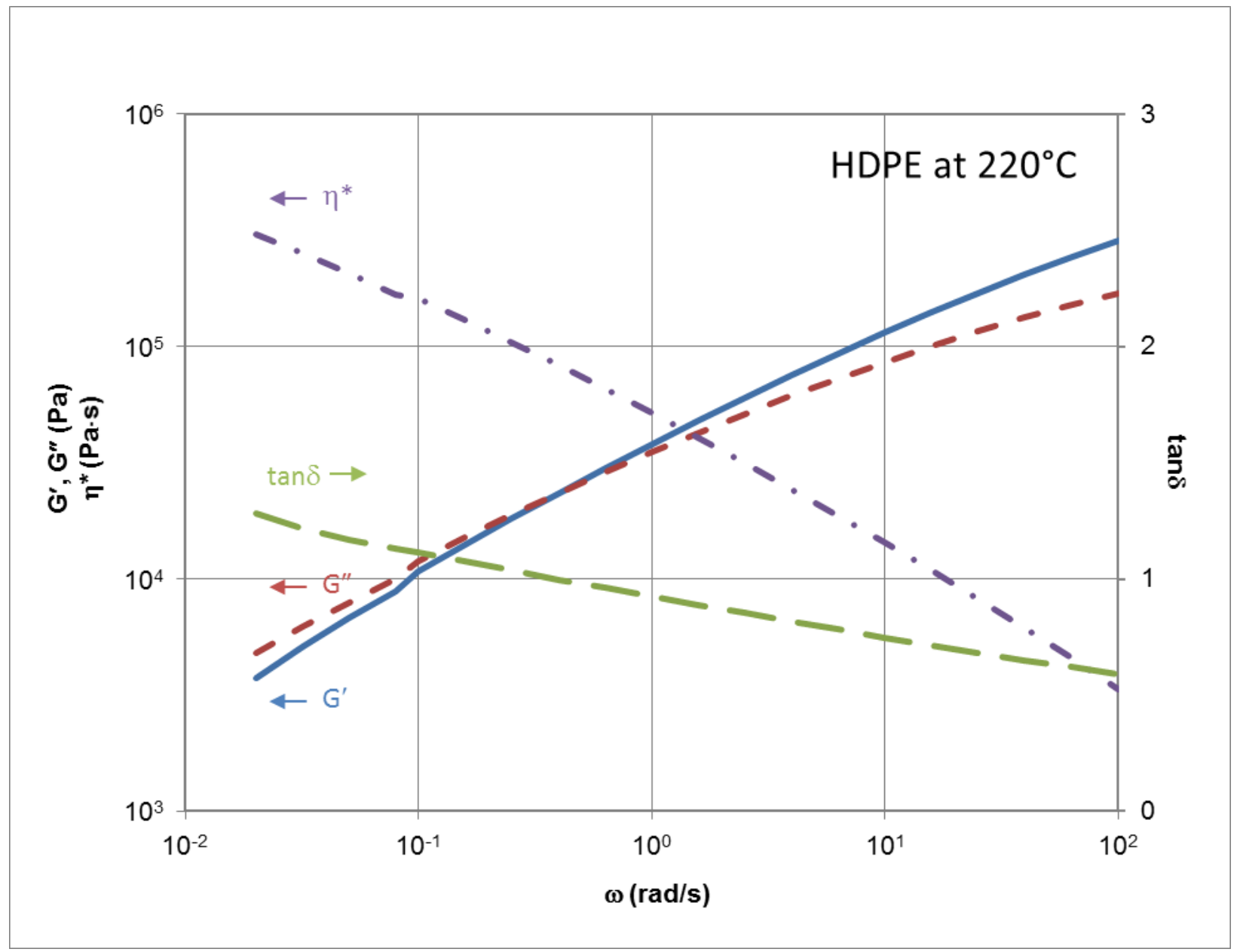

Figure 4-26: Dynamic rheology of HDPE HB-W355-A at $220^{\circ} \mathrm{C}$. Storage modulus $\mathrm{G}^{\prime}$ (solid curve), loss modulus $G^{\prime \prime}$ (dotted curve), complex viscosity $\eta^{*}$ (dash-dot curve), and tangent of phase angle $\tan \delta$ (long dash curve) measured using dynamic frequency sweeps in the linear viscoelastic region. 


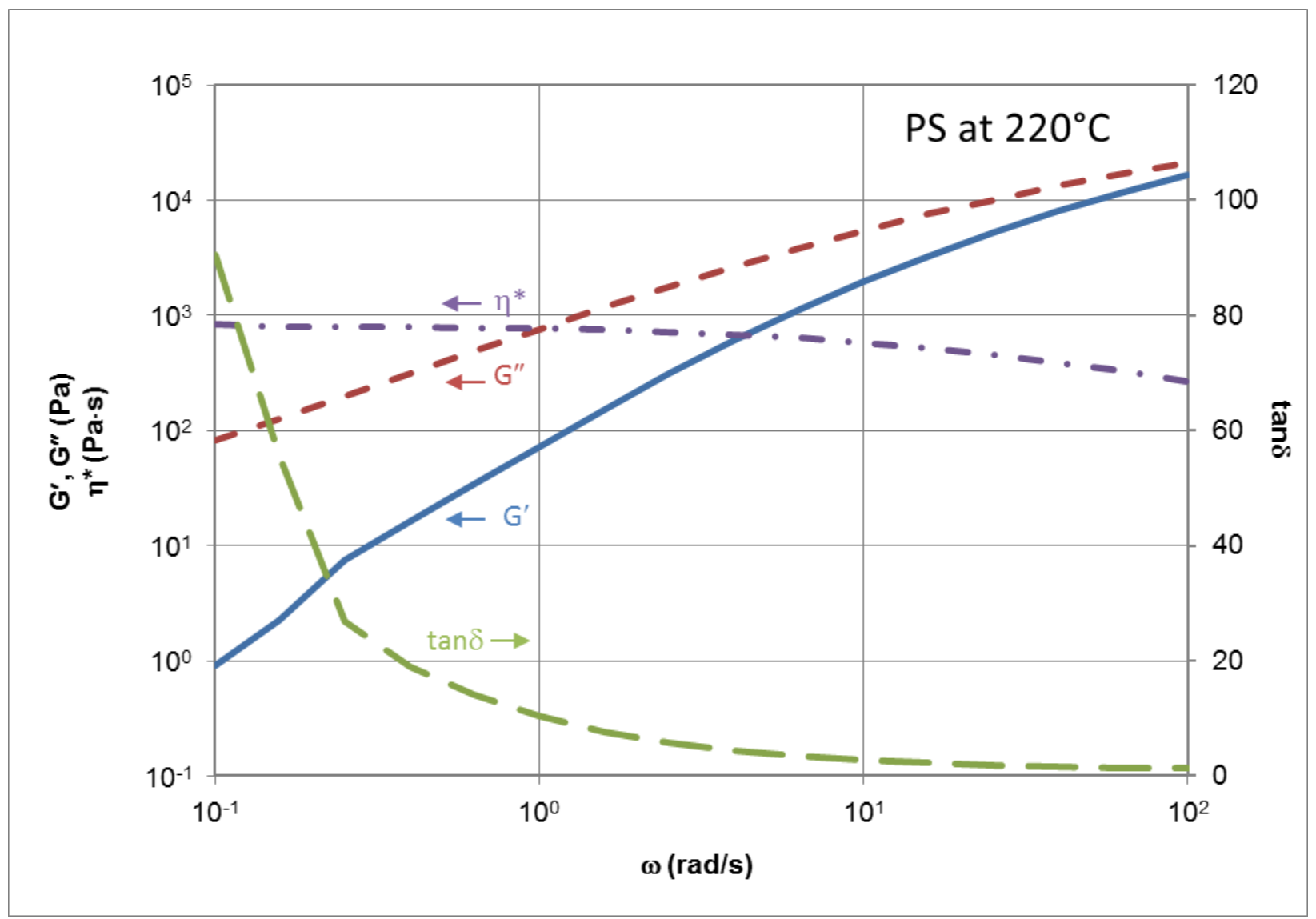

Figure 4-27: Dynamic rheology of PS XU36305 at $220^{\circ} \mathrm{C}$. Storage modulus G' (solid curve), loss modulus G" (dotted curve), complex viscosity $\eta^{*}$ (dash-dot curve), and tangent of phase angle $\tan \delta$ (long dash curve) measured using dynamic frequency sweeps in the linear viscoelastic region. 


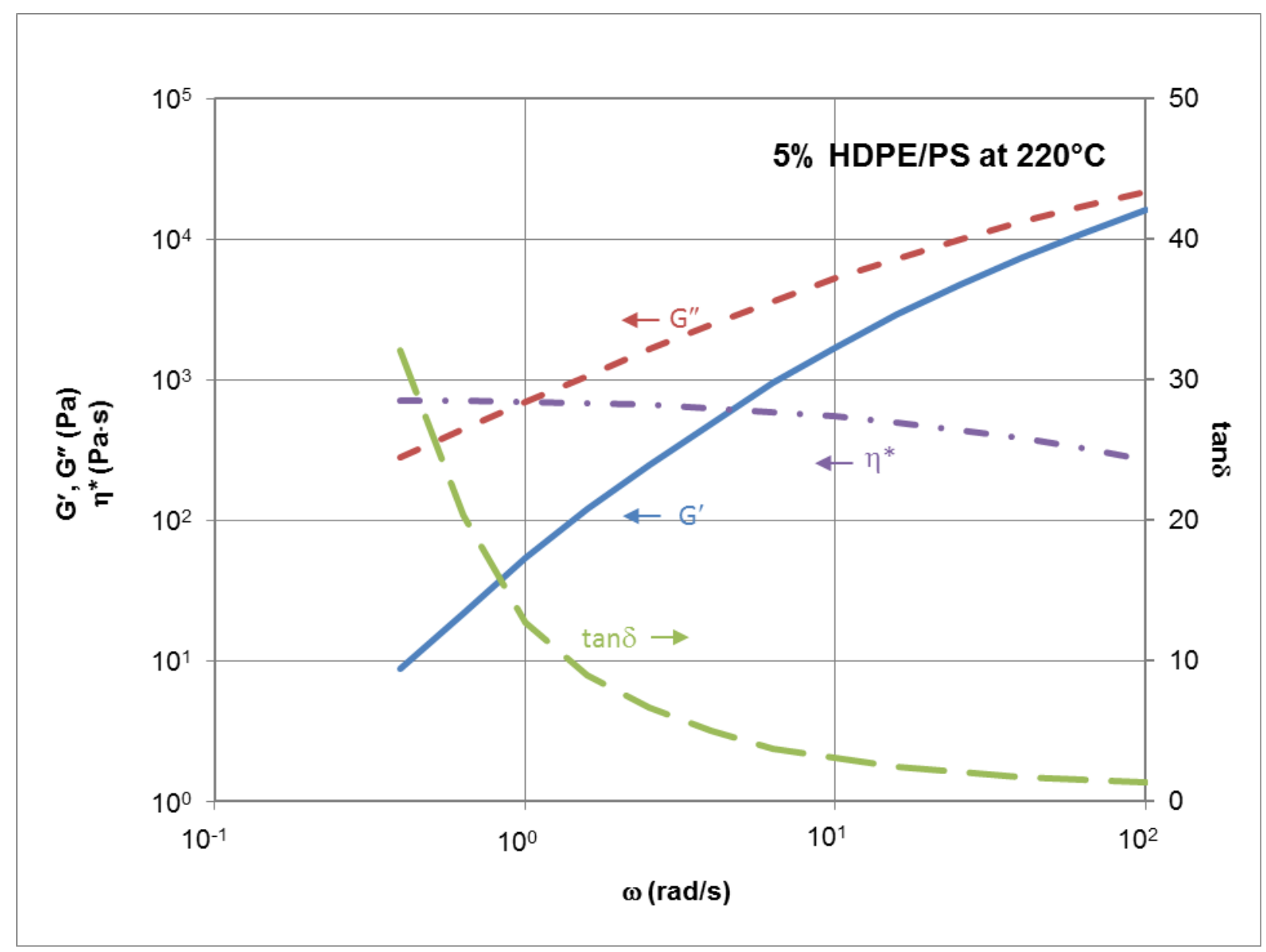

Figure 4-28: Dynamic rheology of $5 \% \mathrm{HDPE} / \mathrm{PS}$ at $220^{\circ} \mathrm{C}$. Storage modulus $\mathrm{G}^{\prime}$ (solid curve), loss modulus G" (dotted curve), complex viscosity $\eta^{*}$ (dash-dot curve), and tangent of phase angle $\tan \delta$ (long dash curve) measured using dynamic frequency sweeps in the linear viscoelastic region.

Table 4-7: Crossover points and relaxation times from rheology measurements

\begin{tabular}{|l|l|c|c|}
\hline Material & $\mathrm{G}_{\mathrm{c}}$ where $\mathrm{G}^{\prime}=\mathrm{G}^{\prime \prime}(\mathrm{Pa})$ & $\omega_{\mathrm{c}}\left(\mathrm{s}^{-1}\right)$ & $\lambda_{\mathrm{r}}(\mathbf{s})$ \\
\hline HDPE HB-W355-A & 22,000 & 0.37 & 2.7 \\
\hline PS XU36305 & $40,000^{*}$ & $500^{*}$ & $0.002^{*}$ \\
\hline $5 \%$ HDPE/PS & $40,000^{*}$ & $800^{*}$ & $0.001^{*}$ \\
\hline
\end{tabular}

*Estimates found by fitting log-transformed $G^{\prime}$ vs. $\omega$ and $G^{\prime \prime}$ vs. $\omega$ with quadratic functions and extrapolating to the crossover point. 


\subsubsection{Interfacial Tension of HDPE/PS}

The appropriate equipment needed to directly measure the surface properties of the constituent materials used in this work was not owned by the Polymer Processing and Characterization Lab and was not identified at WVU. Furthermore, the best methods in measuring the surface properties of polymers, either in the solid or molten phase, are not convenient to measure the surface properties of the nanofillers, and vice versa. A goniometer is commonly used to measure the surface tension of solid polymers. It incorporates a microscope lens to visually observe the stagnant, forward-moving, and trailing contact angles of a drop of liquid with well-defined surface properties on a smooth, flat, and clean surface of the polymer. This use of this method on solid nanofillers is challenging because the nanofiller powder must be pressed into a cake, and the surface is never truly flat on a microscopic level. In fact, true measurement of the surface properties of a solid nanofiller is challenging in general. For these reasons, literature values were used for calculations involving the surface properties of the constituent materials. The challenge with this approach is that the values of surface tension and its disperse and polar parts are very dependent on the methods used in their measurement, and comparisons between different methods typically include a large degree of between-lab variation. For that reason, most of the critical calculations in this work that involved surface properties were repeated using various surface property data.

The surface tension and its components, along with its dependence on temperature, of both HDPE and PS are given in Table 4-8, and the interfacial tension between HDPE and PS is given in Table 4-9. Data presented include those from the well-respected surface tension researcher Souheng $\mathrm{Wu}$ [52] and the average values from the other literature referenced, disregarding molecular weight and molecular branching [53-60]. 
Table 4-8: Surface tension data for HDPE \& PS

\begin{tabular}{|c|c|c|c|c|c|c|c|c|c|c|c|}
\hline \multirow{2}{*}{$\begin{array}{c}\text { Temperature } \\
\left({ }^{\circ} \mathrm{C}\right)\end{array}$} & \multirow{2}{*}{$\begin{array}{c}\text { Reference } \\
\text { Material }\end{array}$} & \multicolumn{5}{|c|}{ Wu 1982} & \multicolumn{5}{|c|}{ Literature Average (All PE \& PS) } \\
\hline & & $\gamma(\mathrm{mN} / \mathrm{m})$ & $x^{p}(\%)$ & $\begin{array}{c}\gamma^{\mathrm{d}} \\
(\mathrm{mN} / \mathrm{m})\end{array}$ & $\begin{array}{c}\gamma^{p} \\
(\mathrm{mN} / \mathrm{m})\end{array}$ & $\begin{array}{c}\mathrm{d} y / \mathrm{dT} \\
{[\mathrm{mN} /(\mathrm{m} \cdot \mathrm{K})]}\end{array}$ & $\gamma(\mathrm{mN} / \mathrm{m})$ & $x^{p}(\%)$ & $\begin{array}{c}\gamma^{d} \\
(m N / m)\end{array}$ & $\begin{array}{c}\gamma^{p} \\
(\mathrm{mN} / \mathrm{m})\end{array}$ & $\begin{array}{c}\mathrm{d} \gamma / \mathrm{dT} \\
{[\mathrm{mN} /(\mathrm{m} \cdot \mathrm{K})]}\end{array}$ \\
\hline \multirow{2}{*}{20} & HDPE & 35.7 & 0.0 & 35.7 & 0.0 & -0.057 & 34.2 & 2.6 & 33.4 & 0.9 & -0.065 \\
\hline & PS (Low MW) & 39.3 & 16.8 & 32.7 & 6.6 & -0.077 & 39.2 & 5.2 & 37.2 & 2.0 & -0.074 \\
\hline \multirow{2}{*}{180} & HDPE & 26.6 & 0.0 & 26.6 & 0.0 & -0.057 & 23.8 & 2.6 & 23.2 & 0.6 & -0.065 \\
\hline & PS (Low MW) & 27.0 & 16.8 & 22.4 & 4.5 & -0.077 & 27.4 & 5.2 & 26.0 & 1.4 & -0.074 \\
\hline \multirow{2}{*}{200} & HDPE & 25.4 & 0.0 & 25.4 & 0.0 & -0.057 & 22.5 & 2.6 & 22.0 & 0.6 & -0.065 \\
\hline & PS (Low MW) & 25.4 & 16.8 & 21.2 & 4.3 & -0.077 & 26.0 & 5.2 & 24.6 & 1.4 & -0.074 \\
\hline \multirow{2}{*}{220} & HDPE & 24.3 & 0.0 & 24.3 & 0.0 & -0.057 & 21.2 & 2.6 & 20.7 & 0.5 & -0.065 \\
\hline & PS (Low MW) & 23.9 & 16.8 & 19.9 & 4.0 & -0.077 & 24.5 & 5.2 & 23.2 & 1.3 & -0.074 \\
\hline
\end{tabular}

\section{Table 4-9: Interfacial tension between HDPE/PS}

\begin{tabular}{|c|c|c|c|c|c|c|}
\hline \multirow[b]{2}{*}{ Temperature $\left({ }^{\circ} \mathrm{C}\right)$} & \multirow{2}{*}{$\begin{array}{c}\text { Reference } \\
\text { Blend }\end{array}$} & \multicolumn{2}{|c|}{ Wu 1982} & \multicolumn{3}{|c|}{ Literature Average (All PE \& PS) } \\
\hline & & $\begin{array}{c}\gamma_{12, \text { harmonic }} \\
\text { mean } \\
(\mathrm{mN} / \mathrm{m})\end{array}$ & $\begin{array}{c}\gamma_{12, \text { geometric }} \\
\text { mean } \\
(\mathrm{mN} / \mathrm{m})\end{array}$ & $\begin{array}{c}\gamma_{12, \text { harmonic }} \\
\text { mean } \\
(\mathrm{mN} / \mathrm{m})\end{array}$ & $\begin{array}{c}\gamma_{12, \text { geometric }} \\
\text { mean } \\
(\mathrm{mN} / \mathrm{m})\end{array}$ & $\begin{array}{c}\gamma_{12, \text { Literature }} \\
\text { average } \\
(\mathrm{mN} / \mathrm{m})\end{array}$ \\
\hline 20 & \multirow{4}{*}{ HDPE/PS } & 6.73 & 6.67 & 0.67 & 0.35 & 8.30 \\
\hline 180 & & 4.88 & 4.71 & 0.49 & 0.25 & 5.45 \\
\hline 200 & & 4.67 & 4.47 & 0.46 & 0.24 & 5.48 \\
\hline 220 & & 4.46 & 4.24 & 0.44 & 0.23 & 5.47 \\
\hline
\end{tabular}


The interfacial tension values used in the calculations in this work for HDPE/PS at $220^{\circ} \mathrm{C}$ are the harmonic-mean value using the literature-average surface tension data, the harmonic-mean value using those from Wu 1982, and the literature-average among the references that specifically listed an interfacial tension value: $0.44 \mathrm{mN} / \mathrm{m}, 4.46 \mathrm{mN} / \mathrm{m}$, and 5.46 $\mathrm{mN} / \mathrm{m}$, respectively.

\subsubsection{Differential Scanning Calorimetry (DSC) of HDPE/PS}

Since HDPE is a very linear molecule, it is semicrystalline with a relatively high crystallinity and rate of crystallization. Therefore, it was of interest to investigate the effects of blending on the crystallinity of HDPE using differential scanning calorimetry (DSC). The DSC curves for the first heating and first cooling for four samples of interest are shown in Figure 4-29; the samples were as-received HDPE, as-received PS, 5\% HDPE/PS coarse blend from the internal mixer, and 5\% HDPE/PS after extensional flow through the converging cone with 0.330 $\mathrm{mm}$ exit diameter at $100 \mathrm{~mm} / \mathrm{min}$ piston speed. The first heating indicates the processing history of the material, including the amount of crystallinity built into HDPE.

The HDPE first heating curve shows a large endothermic melting peak at $T_{\mathrm{m}}=132^{\circ} \mathrm{C}$ corresponding to the melting of crystals formed in the as-received HDPE from its manufacture and physical aging. A measureable cold crystallization peak was not observed for the temperature range of these curves. The $T_{\mathrm{g}}$ of HDPE which can be between -120 and $-20^{\circ} \mathrm{C}[62$ ] is also not measureable within this range. A crystallization exothermic peak is then observed for HDPE upon cooling from the melt, which shows that HDPE crystallizes readily; the cooling rate for this curve was $10^{\circ} \mathrm{C} / \mathrm{min}$. The melting point and crystallinity data for HDPE are summarized in Table 4-10. The heat of fusion for the crystals melting in the melting peak, $\Delta H_{\mathrm{m}}$, is measured by the area of the peak. This value divided by the $\Delta H_{\mathrm{m}, \infty}$ for $100 \%$ crystalline HDPE, which is about $293 \mathrm{~J} / \mathrm{g}$ [63], yields the degree of crystallinity, which was about 54\% for the asreceived material. 
Difficult to see in Figure 4-29 but clearly visible in magnified curves, the PS first heating curve shows a thermal event at $99^{\circ} \mathrm{C}$ corresponding to the $T_{\mathrm{g}}$ of PS. The PS is amorphous, so it does not show a melting peak.

The melting peaks and the corresponding heats of fusion for the $5 \%$ HDPE/PS blends are much smaller than that for HDPE since HDPE is the minor phase. The $\Delta H_{\mathrm{m}}$ was weighted based on the mass fraction of HDPE in the blend by dividing by the mass fraction of HDPE, and those values are comparable to $\Delta H_{\mathrm{m}}$ of the as-received HDPE. Though, the degrees of crystallinity of the HDPE phase in the blends is significantly lower than that of the as-received HDPE. The $T_{m}$ is slightly lower as well. Both of these indicate that the material is not fully crystallized. Indeed, the degrees of crystallinity of the HDPE in the internally mixed coarse blend and in the extensional flow-mixed blend are about $48 \%$ and $46 \%$, respectively. The decrease in crystallinity is expected especially for the extensional flow-mixed blend which is quenched in a water bath after extrusion out of the die. It is also reasonable that the internally mixed coarse blend has similar HDPE crystallinity to the quenched extensional flow-mixed blend because those samples were relatively small with fairly large surface area-to-volume ratios and cooled in air fairly quickly. These results show that HDPE crystallinity is present in the blends created in this work, and HDPE crystallizes quickly with a relatively large amount of crystallinity present even with quenching in water. 


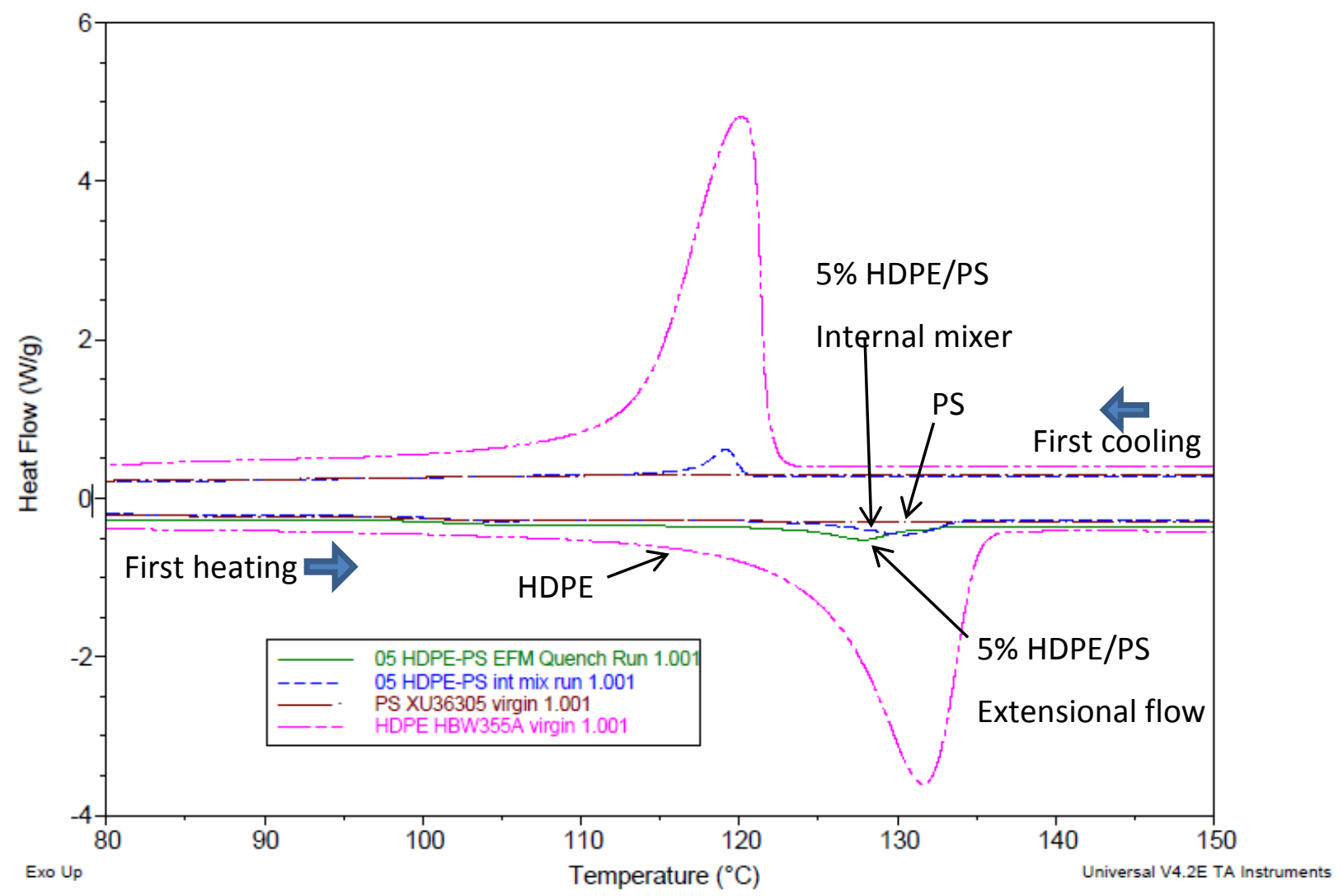

Figure 4-29: DSC curves for HDPE, PS, and blends. 5\% HDPE/PS coarse blend from the internal mixer was cooled in air from the melt, and the $5 \%$ HDPE/PS extensional flow-mixed blend was processed through the converging cone die with $0.330 \mathrm{~mm}$ exit diameter at $100 \mathrm{~mm} / \mathrm{min}$ piston speed and quenched in water. 
Table 4-10: Thermal data for HDPE, PS, and blends

\begin{tabular}{|c|c|c|c|c|}
\hline \multirow[t]{2}{*}{ Material } & PS & \multicolumn{3}{|c|}{ HDPE } \\
\hline & $\mathrm{Tg}_{\mathrm{g}}\left({ }^{\circ} \mathrm{C}\right)$ & $\overline{T_{m}\left({ }^{\circ} \mathrm{C}\right)}$ & $\Delta H_{m}(J / g)$ & Crystallinity (\%) \\
\hline HDPE & - & 131.5 & 158.2 & 54.0 \\
\hline PS & 98.9 & $\mathrm{n} / \mathrm{a}$ & $\mathrm{n} / \mathrm{a}$ & $\mathrm{n} / \mathrm{a}$ \\
\hline $\begin{array}{l}\text { 5\% HDPE/PS } \\
\text { Internal mixer } \\
\text { coarse blend }\end{array}$ & 102.1 & 130.7 & $\begin{array}{l}7.1 \\
\left(141.1^{*}\right)\end{array}$ & 48.2 \\
\hline $\begin{array}{l}5 \% \text { HDPE/PS } \\
\text { Extensional flow } \\
\text { mixed blend } \\
\text { (quenched) }\end{array}$ & 102.2 & 127.7 & $\begin{array}{l}6.7 \\
\left(133.6^{*}\right)\end{array}$ & 45.6 \\
\hline
\end{tabular}

*Values of $\Delta H_{\mathrm{m}}$ weighted by the mass fraction of HDPE in the blend are given in parentheses.

\subsubsection{Conclusions for Blend Selection}

The polymer blend system selected for detailed study in this work was chosen based on the following selection criteria:

1) Physical incompatibility given by a high viscosity ratio for all shear rates the blends are exposed to in processing and

2) Thermodynamic incompatibility given by relatively high interfacial tension, producing coarser blends on which the effects of the techniques employed in this work may be amplified.

A binary blend comprised of a high-molecular weight high-density polyethylene (HDPE) dispersed in a low-molecular weight polystyrene (PS) satisfied these criteria and served as a challenging blend to effectively blend and compatibilize.

Preliminary blends of PC/SAN and PS/LLDPE were investigated based on the materials' availability in the Polymer Processing and Characterization Laboratory, and the preliminary data regarding the mixing time required for preparation of the coarse blends in the internal mixer and first looks at the effects of design and processing parameters on dispersedphase morphology were valuable. The two preliminary blends did not fulfill one of the critical selection criteria, though, that the viscosity ratio be greater than 3.8 for all shear rates 
encountered in the processing of this work. The HDPE/PS blend system, comprised of a highviscosity grade of HDPE dispersed in a low-viscosity grade of PS, did satisfy this requirement and served as a relatively incompatible blend thermodynamically for which the coarse blend was expected to be coarser and offer a better starting point to demonstrate the effects of extensional flow blending on morphology. The results of extensional flow blending of the unfilled HDPE/PS blend system are given in the next section.

\subsection{Extensional Flow Blending of HDPE/PS}

\subsubsection{Converging Cone Dies}

The majority of the investigation into the effects of extensional flow blending on the morphology of HDPE/PS was done using the converging cone dies, described in Section 3.3.2.1. They consist of a straight-walled converging cone nozzle with an entry half-angle of about $47^{\circ}$. The cross section for flow converges from $D_{\mathrm{i}}=11.7 \mathrm{~mm}$ to $D_{\mathrm{o}}=$ either $0.559 \mathrm{~mm}$ or $0.330 \mathrm{~mm}$, corresponding to $\varepsilon=6.1$ or 7.1 , respectively. These converging cone dies differ from the standard dies used in capillary rheometry based on the length of the capillary, which is minimized for the fabricated dies to accentuate the effects of the uniaxial extensional flow and minimize excess shear contributions to the flow profile.

\subsubsection{Effect of Extensional Strain}

The effects of extensional strain in the extensional flow blending 5\% HDPE/PS using the converging cone dies at a given flow rate and temperature are given in Figure 4-30. Extensional flow blending through the converging cone dies significantly decreased $d_{v}$, regardless of extensional strain, but a decrease in $d_{\mathrm{n}}$ required higher extensional strain, with the expense of an increased pressure drop. Both converging cone dies decreased $d_{v}$ by a factor of 2.5 , and the $0.330 \mathrm{~mm}$ cone exit die decreased $d_{\mathrm{n}}$ by a factor of 2.2 , but required a 2.6 times larger pressure drop than the $0.559 \mathrm{~mm}$ cone exit die did. The effective decrease in $d_{\mathrm{v}}$ is also observed in the discrete histograms and representative SEM images, in which one may observe that the large 
HDPE drops present in the coarse blend were broken up in the extensional flow-blended systems.

The residence time in the converging cone section, $t_{\text {res, }}$ and the average extensional strain rate, $\bar{\varepsilon}$, are given in Figure 4-30 as well. They may be calculated based on the following equations [64]:

$$
\begin{gathered}
t_{\text {res }}=\int_{0}^{L} \frac{d z}{v_{\mathrm{z}}}=\frac{L\left(R_{\mathrm{i}}^{2}+R_{\mathrm{i}} R_{\mathrm{o}}+R_{\mathrm{o}}^{2}\right)}{3 v_{\mathrm{i}} R_{\mathrm{i}}^{2}} \\
\overline{\dot{\varepsilon}}=\frac{\varepsilon}{t_{\text {res }}},
\end{gathered}
$$

where $L$ is the length in the flow direction, or $z$ direction, from the entrance, subscript $i$, to the exit, subscript $\mathrm{o}$, of the convergence, and $v_{\mathrm{i}}$ and $R_{i}$ are the flow velocity in the $z$ direction at the entrance and radius of the cross-sectional area for flow at either the entrance or exit, respectively. Equation (4-10) may also be expressed in terms of the entrance and exit diameters, $D_{i}=2 R_{i}$, the volumetric flowrate $Q=A_{\mathrm{c}, \mathrm{i}} v_{\mathrm{i}}=\pi / 4 \cdot D_{\mathrm{i}}^{2} v_{\mathrm{i}}$, and the entry half-angle of the convergence, that is the angle between the centerline axis and the converging cone wall, $\alpha$ :

$$
\begin{aligned}
t_{\text {res }} & =\frac{\pi\left(D_{\mathrm{i}}^{3}-D_{\mathrm{o}}^{3}\right)}{24 Q \tan \alpha}, \\
\text { where } \tan \alpha & =\frac{R_{\mathrm{i}}-R_{\mathrm{o}}}{L}=\frac{D_{\mathrm{i}}-D_{\mathrm{o}}}{2 L} .
\end{aligned}
$$

The residence time is shorter than the relaxation time of the HDPE dispersed phase, as given in Table 4-7, and so transient effects and elasticity are expected to play important roles in the behavior of the system. Furthermore, the difference between the average extensional strain rates for the two converging cone dies is not great, and the difference in the two dies' performance is likely due to the difference in total extensional strain rather than extensional strain rate. 


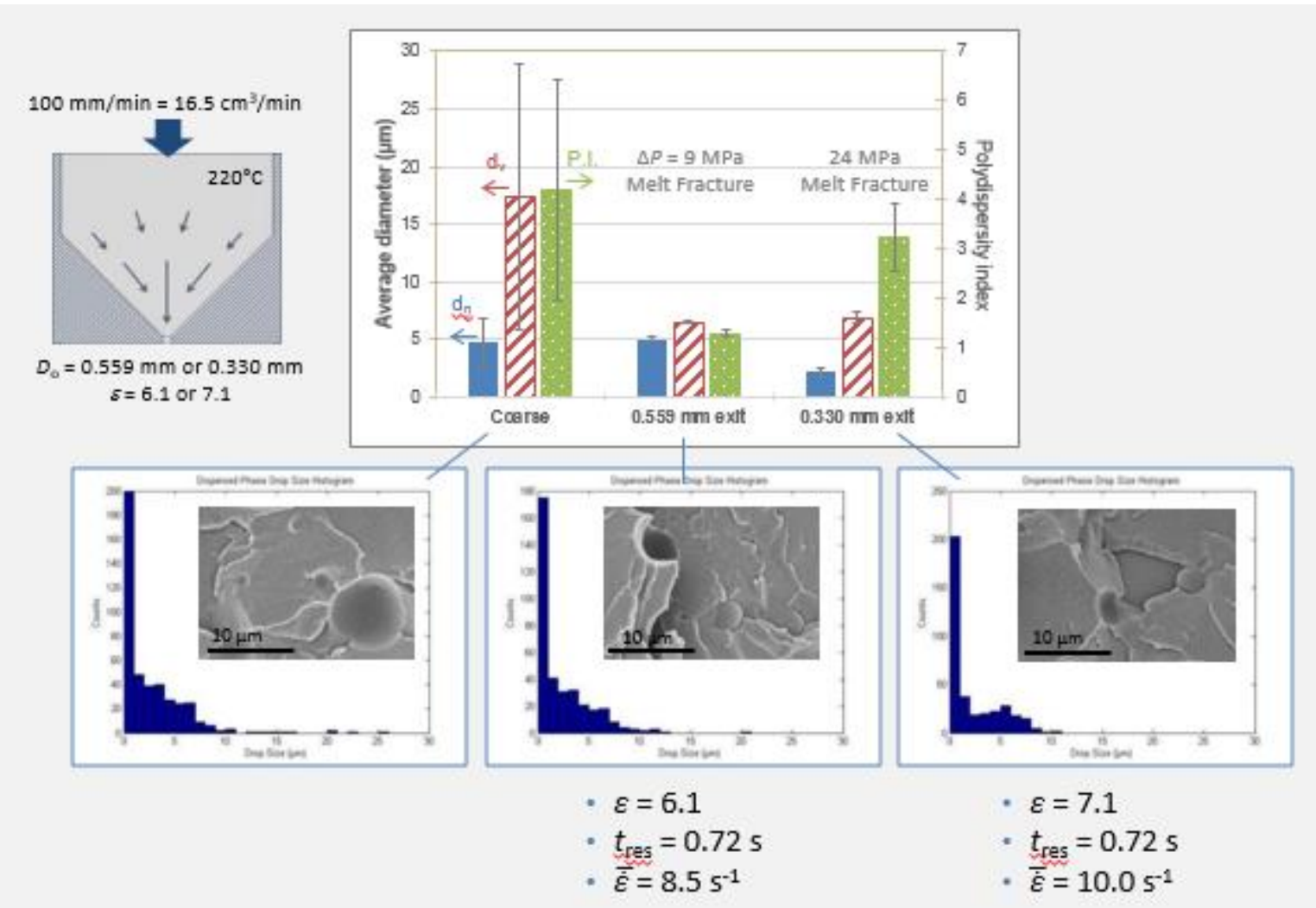

Figure 4-30: Effect of extensional strain in converging cone dies on morphology. Morphology of unfilled 5\% HDPE/PS before and after extensional flow blending using converging cone dies along with the pressure drop for each. The extensional strains for the two converging cone dies were 6.1 and 7.1 for the $0.559 \mathrm{~mm}$ and $0.330 \mathrm{~mm}$ exit diameters, respectively. Temperature was $220^{\circ} \mathrm{C}$ and piston speed was $100 \mathrm{~mm} / \mathrm{min}$, equating to a $16.5 \mathrm{~cm}^{3} / \mathrm{min}$ flow rate. Error bars represent \pm 1 standard deviation across 3 replicate cross sections. Morphology is also represented by discrete histograms and representative SEM images.

To compare to the theory presented in Section 2.2, the capillary number, Ca, associated with each of the flows and morphologies before and after extensional flow blending through the converging cone dies is plotted in Figure 4-31. The solid curves represent $\mathrm{Ca}_{\text {crit, }}$, from the quasi-steady state, binary breakup of an isolated initially spherical, Newtonian drop in a Newtonian matrix, for simple shear flow (upper) and planar extensional flow (lower), and the

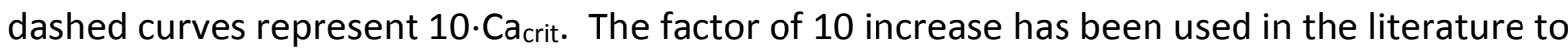
account for the extenuating circumstances present in real processing that break the assumptions of the $\mathrm{Ca}_{\text {crit }}$ measurements, including transient, mixed flow fields, viscoelastic, non-isolated, and non-binary breakup effects. However, progress toward truly extended the 
capillary number analysis to account for these effects has had limited acceptance in the field, and the factor of 10 is not a robust factor to account for all of these effects. This is the hypothesis to why the Ca values plotted for the flow histories and morphologies before and after extensional flow blending through the converging cone dies are much higher than even 10. $\mathrm{Ca}_{\text {crit. }}$ Indeed, $\mathrm{Ca}$ is about 10 fold larger than $10 \cdot \mathrm{Ca}_{\text {crit }}$ and 100 fold larger than $\mathrm{Ca}_{\text {crit. }}$. The transient and short-lived nature of the uniaxial extensional flow, coupled with the elasticity of the high-molecular weight dispersed polymer phase, is likely the primary source of the disparity.

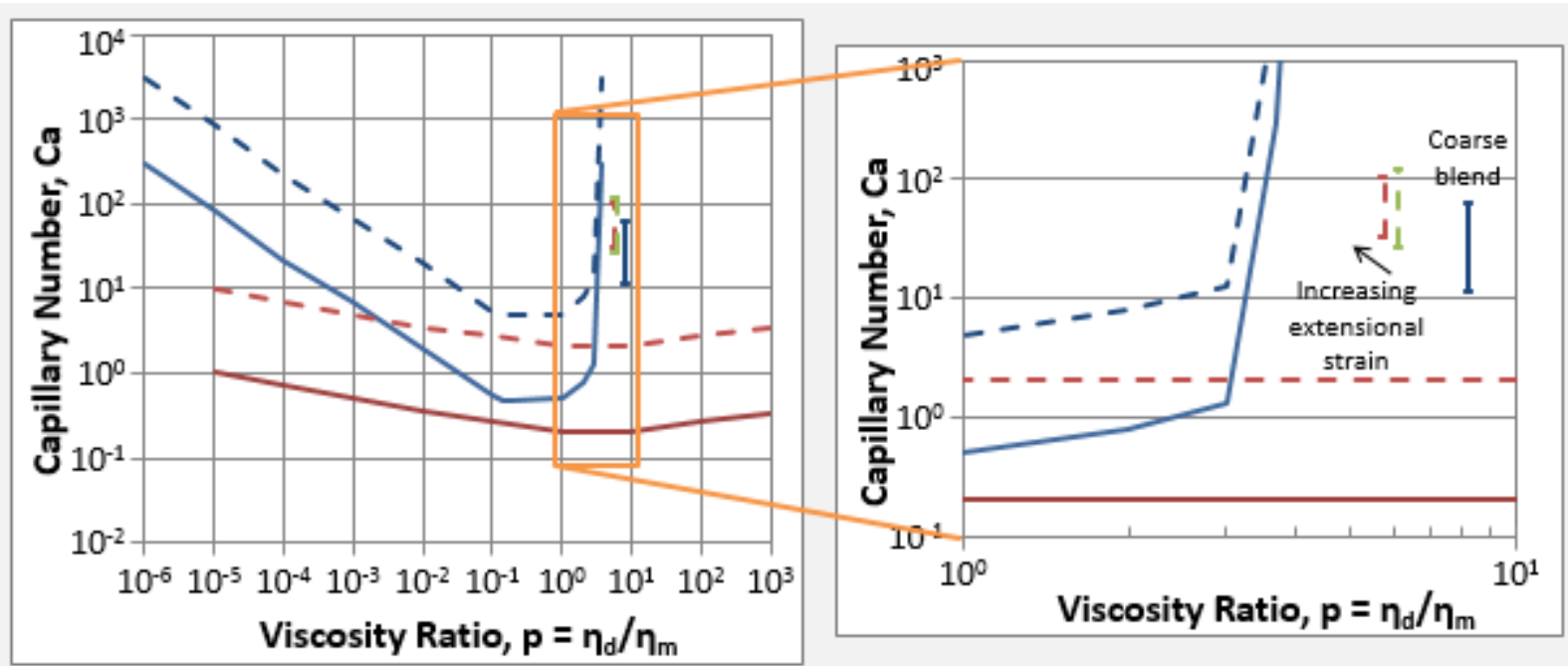

Figure 4-31: Effect of total strain on Ca for converging cone dies. Capillary number for each flow history and morphology, where the lower end of the bar corresponds to $d_{\mathrm{n}}$ and the upper end to $d_{v}$. The solid curves represent $\mathrm{Ca}_{\text {crit }}$ for simple shear flow (upper) and planar extensional flow (lower), and the dashed curves represent 10.Cacrit. Left - full curve and Right-magnified inset of the plot.

To investigate further, other dimensionless numbers are useful. Namely the Deborah number, De, and Weissenberg number, Wi, of Equations (4-8) and (4-9), are useful to compare the time scales associated with the extensional flow to the elastic relaxation time of the HDPE dispersed phase. The relaxation time was estimated from the dynamic rheology, and that of the HDPE phase is given in Table 4-7 as $2.7 \mathrm{~s}$. This yields $\mathrm{De}=3.8$ for both converging cone dies, and $\mathrm{Wi}=22.9$ and 26.1 for the converging cone dies with exit diameters of $0.559 \mathrm{~mm}$ and 0.330 
$\mathrm{mm}$, respectively. These are given in Table 4-11. The Wi being much greater than unity implies that the extensional strain rate through the die is much higher than the relaxation time. This would be acceptable if extensional strain fields were not so short-lived; the total extensional strain then becomes important. Wi/De becomes $\dot{\varepsilon} t_{\text {res }}=\varepsilon$, which is large enough in both dies to have a significant effect, but larger strains are possible when the extensional flow field is able to be applied over a greater volume, like in planar hyperbolic extensional flow.

Table 4-11: Dimensionless numbers for extensional strain through converging cone dies

\begin{tabular}{|c|c|c|}
\hline $\begin{array}{l}\text { Converging cone exit diameter } \\
(\mathrm{mm})\end{array}$ & 0.559 & 0.330 \\
\hline$\varepsilon$ & 6.08 & 7.14 \\
\hline p using $\eta_{\text {power-law,wall }}$ & 5.1 & 4.5 \\
\hline $\begin{array}{l}C a_{s} \text {, using } d_{n} \& \eta_{\text {power-law,wall and }} \\
\text { using } d_{v} \text { and } \eta_{0} \\
\left(C a_{s} / C a_{c r i t}\right)\end{array}$ & $\begin{array}{r}170-10,000 \\
(770-40,000)\end{array}$ & $\begin{array}{r}130-51,000 \\
(610-210,000)\end{array}$ \\
\hline $\begin{array}{l}\text { Re, using } d_{v} \text { and } \eta_{0} \text { and using } d_{n} \\
\text { and } \eta_{\text {power-law,wall }}\end{array}$ & $8 \times 10^{-5}-5 \times 10^{-3}$ & $7 \times 10^{-5}-3 \times 10^{-2}$ \\
\hline $\mathrm{t}_{\text {res }}(\mathrm{s})$ & 0.72 & 0.72 \\
\hline $\bar{\varepsilon}\left(s^{-1}\right)$ & 8.5 & 10.0 \\
\hline $\begin{array}{l}C a_{e} \text {, using } d_{n} \text { and using } d_{v} \text {, both } \\
\text { with } \eta_{e}=3 \eta_{0} \\
\left(C a_{e} / C a_{c r i t}\right)\end{array}$ & $\begin{array}{r}12-16 \\
(50-60)\end{array}$ & $\begin{array}{r}6-20 \\
(25-80)\end{array}$ \\
\hline $\mathrm{De}$ & 3.8 & 3.8 \\
\hline $\mathrm{Wi}$ & 22.9 & 26.9 \\
\hline
\end{tabular}

Also given in Table 4-11 are the calculated capillary numbers, both for shear and extension; the minimum and maximum calculated values are each given because the calculated Ca depends strongly on the morphology quantification metric and constitutive rheological model used. In addition, concern was raised over the appropriate interfacial tension value to use, as discussed in Section 4.1.3.2, and for these calculations, the harmonic-mean interfacial tension using the surface properties from Wu 1982 [52] was used, $\gamma_{12}=4.46 \mathrm{mN} / \mathrm{m}$. The calculated $\mathrm{Ca}_{\mathrm{s}}$ values were 2-5 orders of magnitude greater than $\mathrm{Ca}_{\text {crit }}$ for planar extensional flow. The critical capillary number $\mathrm{Ca}_{\text {crit }}$ is given in equation form by Starý et al. [65]. The 
reasons for the disparity between $\mathrm{Ca}_{\mathrm{s}}$ and $\mathrm{Ca}_{\text {crit }}$ are many because the flow field, breakup mechanism, transient nature of the flow, non-isolated drops, and phase constitutive rheology are all different than those used to measure $\mathrm{Ca}_{\text {crit. }}$. The largest likely sources of the disparity between $\mathrm{Ca}_{\mathrm{s}}$ and $\mathrm{Ca}_{\text {crit }}$ are the transient nature of the uniaxial extensional flow field and the viscoelasticity of the HDPE dispersed phase. The differential also implies that there remains a driving force, the $\Delta$ between $\mathrm{Ca}_{\mathrm{s}}$ and $\mathrm{Ca}_{\text {crit, }}$, for further breakup of the dispersed phase.

The extensional capillary number, however, showed a much lower differential between $\mathrm{Ca}_{\mathrm{e}}$ and $\mathrm{Ca}_{\text {crit. }}$. This is because the magnitude of the average extensional strain rate $\overline{\dot{\varepsilon}}$ is much smaller than that of the shear rate $\dot{\gamma}$ and the extensional viscosity is larger than the shear viscosity. For this work, the extensional viscosity was estimated assuming Newtonian behavior, using a Trouton ratio of 3 , that is $\eta_{\mathrm{e}}=3 \eta_{\mathrm{s}}$. The true extensional viscosity is likely larger due to the viscoelasticity of the HDPE dispersed phase, and extensional strain hardening may further cause deviation from Newtonian behavior. Since the converging cone region's flow fields are dominated by extensional flow, $\mathrm{Ca}$ and the $\Delta$ between $\mathrm{Ca}_{\mathrm{e}}$ and $\mathrm{Ca}_{\text {crit }}$ are the important flow metrics, and the differential between them is likely explained by the transient nature of the flow fields and the viscoelastic constitutive rheology of the polymers.

Finally, the range of Reynolds numbers associated with the flow around a dispersed HDPE drop is listed in Table 4-11. The very low Re values imply that the flow is creeping flow in which inertial effects are negligible compared to viscous effects. This is common in polymer processing due to the large viscosities of polymer melts and solutions. This is also a good indicator that buoyant effects due to gravity are likely negligible. The density ratio, $\omega_{\rho}$, of the HDPE dispersed phase over the PS matrix phase is given by the following:

$$
\omega_{\rho}=\frac{\rho_{\mathrm{d}}}{\rho_{\mathrm{m}}}
$$

where $\rho_{\mathrm{d}}$ and $\rho_{\mathrm{m}}$ are the melt densities of the dispersed and matrix phases, respectively. The melt densities were approximated by the solid densities, assuming incompressible flow, and the density ratio between HDPE and PS is 0.91 . Therefore, even if buoyant effects were important, the density ratio is not far from unity. 


\subsubsection{Morphological Comparisons to the Literature}

Before moving on to study the effects of other influential factors, it is prudent to compare to the morphologies obtained for similar immiscible polymer blends in the literature. Blending HDPE and PS with very similar shear viscosities as those used in this work, Min et al. [66] observed phase sizes of the same order of magnitude but slightly smaller than the phase sizes seen in this work. For $10 \mathrm{wt} \% \mathrm{HDPE} / \mathrm{PS}$, they observed $d_{\mathrm{n}}=1.3-1.8 \mu \mathrm{m}$ and $d_{\mathrm{w}}=1.4-2.6$ $\mu \mathrm{m}$, where $d_{\mathrm{w}}$ is the weight-average diameter:

$$
d_{\mathrm{w}}=\frac{\sum_{i=1}^{n} N_{i} d_{i}^{2}}{\sum_{i=1}^{n} N_{i} d_{i}},
$$

after blending using a lab-scale Brabender single-screw extruder with a KMB-100 Koch static mixer to impart distributive blending onto the blend. This may be compared to the values observed in Figure 4-30 for the coarse blend and the blends after extensional flow blending through the converging cone dies at $17 \mathrm{~cm}^{3} / \mathrm{min}$, which are tabulated in Table 4-12 along with the metrics using the fitted log-normal distribution for each of the discrete histograms. The values obtained in this work are slightly larger, except for the blend after processing through the converging die with $0.330 \mathrm{~mm}$ exit diameter, $\varepsilon=7.1$. Min et al. also processed their $10 \%$ HDPE/PS blend through a capillary die with a diameter of $1.5 \mathrm{~mm}$ and a length-to-diameter, $L / D$, ratio of 40 and observed $d_{\mathrm{n}}=1.59 \mu \mathrm{m}$ and $d_{\mathrm{w}}=1.72 \mu \mathrm{m}$. This large $L / D$ ratio die accentuates shear effects and makes entrance and exit effects, where extensional flow fields are present, negligible. The authors also compare to prior literature and show that the phase sizes that they observed are comparable to those of the literature at the time. 
Table 4-12: Morphology using converging cone dies at $17 \mathrm{~cm}^{3} / \mathrm{min}$. Listed are two different methods to quantify the morphology of each blend, listing the mean \pm 1 standard deviation among 3 replicate cross sections.

\begin{tabular}{|l|c|c|c|c|c|}
\hline \multicolumn{1}{|c|}{ Blending history } & $d_{n}(\mu \mathrm{m})$ & $d_{v}(\mu \mathrm{m})$ & \multicolumn{1}{|c|}{ P.I. } & $\begin{array}{c}\mu_{\text {log-normal }} \\
(\mu \mathrm{m})\end{array}$ & $\begin{array}{c}\text { Olog-normal } \\
(\mu \mathrm{m})\end{array}$ \\
\hline $\begin{array}{l}\text { Coarse blend from } \\
\text { internal mixer }\end{array}$ & $4.7 \pm 2.2$ & $17.4 \pm 11.6$ & $4.2 \pm 2.2$ & $3.5 \pm 2.5$ & $3.0 \pm 1.3$ \\
$\begin{array}{l}\text { Blend after flow at 17 } \\
\mathrm{cm}^{3} / \text { min through } \\
\text { converging cone die } \\
\text { with 0.559 mm }(\varepsilon=6.1)\end{array}$ & $5.0 \pm 0.2$ & $6.5 \pm 0.1$ & $1.3 \pm 0.1$ & $4.7 \pm 0.2$ & $1.5 \pm 0.1$ \\
\hline $\begin{array}{l}\text { Blend after flow at 17 } \\
\mathrm{cm}^{3} / \text { min through } \\
\text { converging cone die } \\
\text { with } 0.559 \text { mm }(\varepsilon=6.1)\end{array}$ & $2.2 \pm 0.3$ & $6.9 \pm 0.5$ & $3.2 \pm 0.7$ & $0.9 \pm 0.2$ & $4.4 \pm 0.3$ \\
\hline
\end{tabular}

The morphology results herein are also consistent with other works in the literature. Utracki and Sammut [51] performed controlled coalescence experiments on blends of 33.33 wt\% PS/LDPE. The average drop diameter, $d_{\mathrm{n}}$, was $3 \pm 0.5 \mu \mathrm{m}$ for their blends initially prepared on a twin-screw extruder, and it was observed to increase to about $8 \mu \mathrm{m}$ after controlled shearflow coalescence. With increasing shear rate, the coalescence effect also caused the formation of short fibrils of the dispersed PS phase. This is commonly observed in practice but was avoided in the work of this dissertation by using relatively dilute dispersed-phase concentrations. The authors also investigated the compatibilization effect of adding a block copolymer, poly[styrene-b-(ethylene-co-butylene)] (SEB). This yielded an initial drop diameter $d_{\mathrm{n}}=1.4 \pm 0.3 \mu \mathrm{m}$, which increased to $2.8 \pm 0.2 \mu \mathrm{m}$ after shear flow, and further coarsened into short fibrils about $4 \mu \mathrm{m}$ in diameter with $L / D$ of about 5 with increased shearing.

Compatibilization using block copolymers is very common in the academic literature and its prevalence in commercial offerings is growing. Li and Favis [67] found the volume-average diameter of dispersed PS drops decrease to sub-micron levels with compatibilization with poly(styrene-co-ethylene butylene-co-styrene) (SEBS) for blends of $5 \mathrm{wt} \%$ PS/HDPE with 12.5 wt\% SEBS identified as the critical concentration required to saturate the PS/HDPE interface and stabilize the morphology to an equilibrium volume-average diameter of $0.98 \mu \mathrm{m}$. They 
observed reductions in $d_{\mathrm{v}}$ by factors of 1.6 to 3.1 to $d_{\mathrm{v}}=0.58 \mu \mathrm{m}$ to $1.41 \mu \mathrm{m}$ over a range of dispersed PS phase concentrations of $1 \%$ to $30 \%$. Additionally, for the inverse blend, 12.5 wt\% SEBS was also critical to reduce $d_{v}$ by factors of 2.2 to 4.2 , yielding $d_{v}=0.68-0.70 \mu \mathrm{m}$, and this effect was very consistent for dispersed HDPE phase concentrations of $5 \%, 10 \%$, and $20 \%$ in PS matrices.

Chen and White [68] observed similar reductions in the dispersed-phase size of HDPE/PS blends with compatibilization with SEBS. They found $d_{w}=0.9-2.2 \mu \mathrm{m}$ over the entire concentration range from 20\% HDPE/PS to $20 \%$ PS/HDPE, and this decreased to $0.3-0.55 \mu \mathrm{m}$ with $5 \%$ SEBS added to the blends. Therefore, the conclusion may be drawn that for HDPE/PS, the dispersed-phase size associated with true compatibilization is sub-micron, about $0.5 \mu \mathrm{m}$, in diameter. This is achieved using block copolymer compatibilizers such as SEBS, which is available commercially through Kraton Corporation, formerly a division of Shell Chemical Company. Blends of HDPE dispersed in PS with SEBS compatibilizer may be used in the industry as weatherable high-impact polystyrene (HIPS) grades.

Sub-micron level dispersion was not achieved in the work of this dissertation. Though, the phase sizes observed in this work are consistent with those in the literature that do not use block copolymer or reactive copolymer compatibilization. Furthermore, few works in the literature study blends with such high viscosity ratios as those investigated in the work of this dissertation.

\subsubsection{Effects of Flow Rate \& Extensional Strain Rate}

The effects of flow rate through the converging cone die are shown in Figure 4-32 where the converging cone with $0.330 \mathrm{~mm}$ exit diameter and $\varepsilon=7.1$ was used with a constant temperature of $220^{\circ} \mathrm{C}$. With increasing flow rate, and its concomitant increase in extensional strain rate, both $d_{\mathrm{v}}$ and $d_{\mathrm{n}}$ decrease and reach a minimum at a piston speed of $100 \mathrm{~mm} / \mathrm{min}$, corresponding to a flow rate of $17 \mathrm{~cm}^{3} / \mathrm{min}$ and an extensional strain rate of $10 \mathrm{~s}^{-1}$. For faster

flow rates, $d_{\mathrm{v}}$ appears to remain the same, but $d_{\mathrm{n}}$ appears to increase slightly compared to that using the $17 \mathrm{~cm}^{3} / \mathrm{min}$ flow rate. This optimum behavior has been observed in the literature and may be explained by one or several phenomena. 

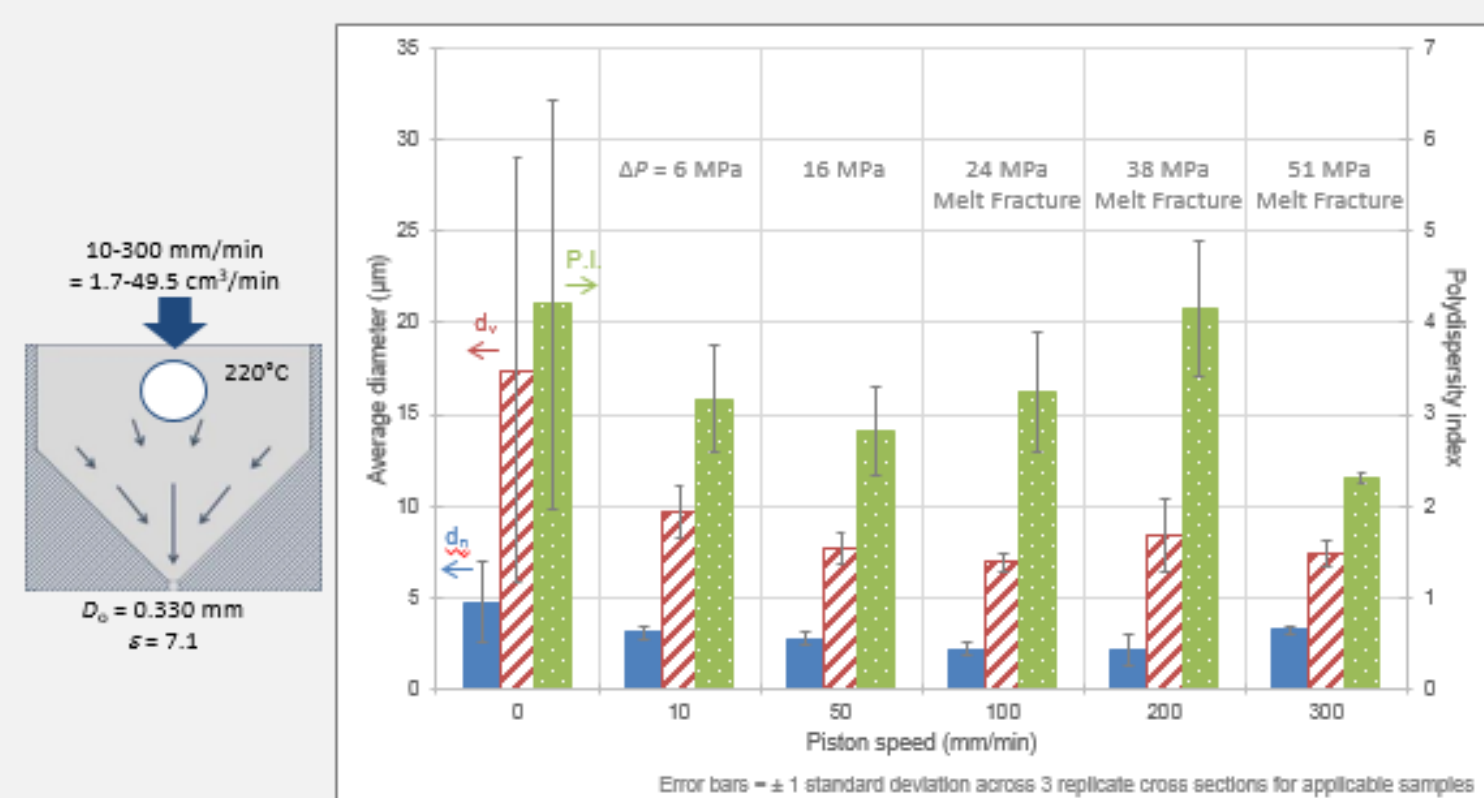

\begin{tabular}{|l|l|l|l|l|l|}
\hline$Q\left(\mathrm{~cm}^{3} / \mathrm{min}\right)$ & 2 & 8 & 17 & 33 & 50 \\
\hline$t_{\text {res }}(\mathrm{s})$ & 7.2 & 1.4 & 0.7 & 0.4 & 0.2 \\
\hline $\bar{\varepsilon}\left(\mathrm{s}^{-1}\right)$ & 1 & 5 & 10 & 20 & 30 \\
\hline
\end{tabular}

Figure 4-32: Effect of flow rate through converging cone dies on morphology

The most intuitive explanation for the presence of an optimum with respect to flow rate is that based on the discussion of De and Wi as in Section 4.2.1.1. When the residence time in the convergence becomes significantly shorter than the elastic relaxation time of the dispersed phase, then drop deformation is truncated well short of its equilibrium elongation, even though the extensional strain rate is high. The elastic HDPE dispersed phase does not have enough time of exposure to the uniaxial extensional flow fields to fully elongate and breakup when the flow rate is too fast. Furthermore, elasticity of the dispersed phase by itself contributes to breakup, and its effects predict an optimum flow rate for maximum drop deformation and breakup [16]. Extensional strain hardening may become pronounced at high extensional strain rates as well, which retards drop deformation and breakup to a point where the morphology remains consistent even with increasing flow rate and extensional strain rate [69]. 
Unfortunately, the extensional rheology of the polymers used in this work was not measured, so further investigation into these effects is reserved for future work.

Another factor is important to the overall behavior of the molten polymer system and likely plays a critical role in the optimum morphological behavior with respect to flow rate, and that is melt fracture. In Figure 4-32, notation is included for those extensional flow-blended extrudates that showed melt fracture. Melt fracture corresponds to a range of flow instabilities that arise in polymer processing, including extrudate surface distortions or shark-skinning and gross extrudate fracture in which the extrudate spirals either regularly or randomly. These instabilities, among others, have been reviewed by Larson [70]. No distinction was given in the notations of this work between the onset of melt fracture, at which flow surging causes surface distortions, or shark-skinning, and gross melt fracture. The onset of melt fracture is typically observed at a shear stress at the wall on the order of $10^{5} \mathrm{~Pa}$, and it is believed to involve periodic slippage at the wall, that is the non-slip boundary condition breaks down, due to fluid elasticity. The optimum flow rate of Figure $4-32$ at $17 \mathrm{~cm}^{3} / \mathrm{min}$ is also the first flow rate where melt fracture was observed. This was observed in the literature as well where the optimal flow rate through converging dies with various entry profiles was always the one just before the onset of melt fracture [71].

In that work, Meller et al. studied converging dies with various entry profiles, including those with entry half-angles of $90^{\circ}, 60^{\circ}, 45^{\circ}$, and $30^{\circ}$ as well as one with a hyperbolic profile, or trumpeted shape, for which the extensional strain rate is constant at all points in the convergence; these are shown in Figure 4-33. The authors concluded that the onset of gross melt fracture limited the maximum flow rate that was effective at dispersing a high-viscosity ratio blend. Apparently the flow instabilities associated with gross melt fracture disrupt the flow profile in the convergence and adversely affect drop deformation and breakup compared to flows without melt fracture. They hypothesize that optimum breakup of the dispersed polymer phase occurs for the maximum volume in the convergence where the Weissenberg number exceeds $0.5(\mathrm{Wi}>0.5)$ and where the extensional stress imparted onto the drops is greater than the critical stress required for breakup. The first condition, $\mathrm{Wi}>0.5$, has been reported in the literature as the condition for drop breakup due to elastic fracture with large 
enough extensional strain rate, and the latter condition implies that the total extensional stress imparted onto the drop is also important. This hypothesis was validated in their work for all of the dies, with the $30^{\circ}$ entry half-angle and the trumpeted dies yielding the maximum dispersive effects. However, for very high-viscosity ratio blends, $p>100$, the trumpeted die did not yield optimal dispersive mixing results, likely because the die with hyperbolic entry profile yields a large volume where the extensional stress is large, but does not yield a large volume where the extensional strain rate is high due to the die's gradual convergence.

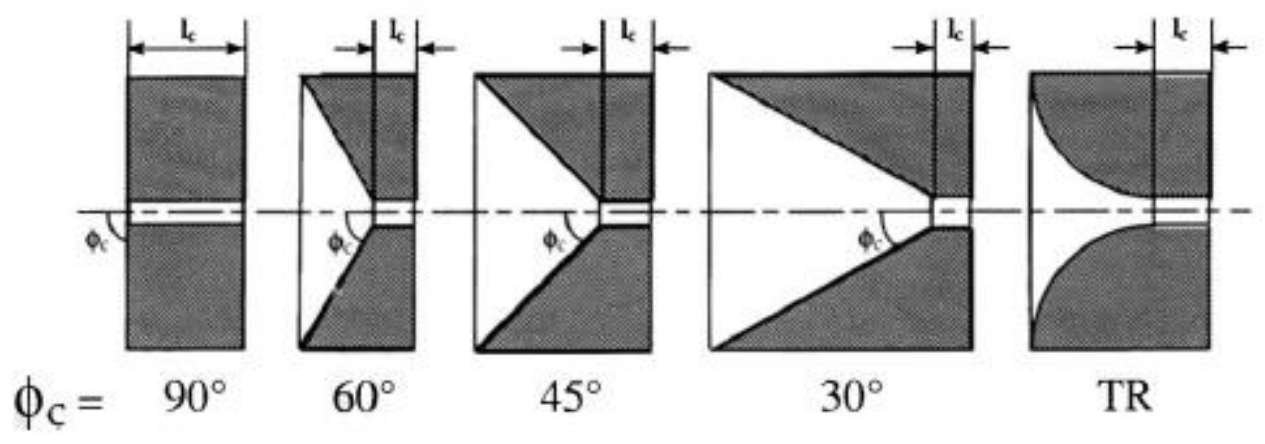

Figure 4-33: Entry profiles studied by Meller et al. 2002 (Reproduced with permission from Meller et al. 2002 [71]). The angles notated are those of the entry half-angles, and the rightmost convergence has a hyperbolic profile, or trumpeted shape.

Figure 4-34 shows the shear capillary number, $\mathrm{Ca}$ or $\mathrm{Ca}_{\mathrm{s}}$, for the blends before and after extensional flow blending through the converging cone die with increasing flow rate. As in Figure 4-31, the $\mathrm{Ca}$ is much larger than $\mathrm{Ca}_{\text {crit, }}$, and the difference, or $\Delta$, between $\mathrm{Ca}$ and $\mathrm{Ca}_{\text {crit }}$ appears to be proportional to the flow rate, corroborating the assumption that increasing flow rate accentuates the transient and viscoelastic effects in the uniaxial extensional-flow processing that invalidate its comparisons to $\mathrm{Ca}_{\text {crit. }}$. 


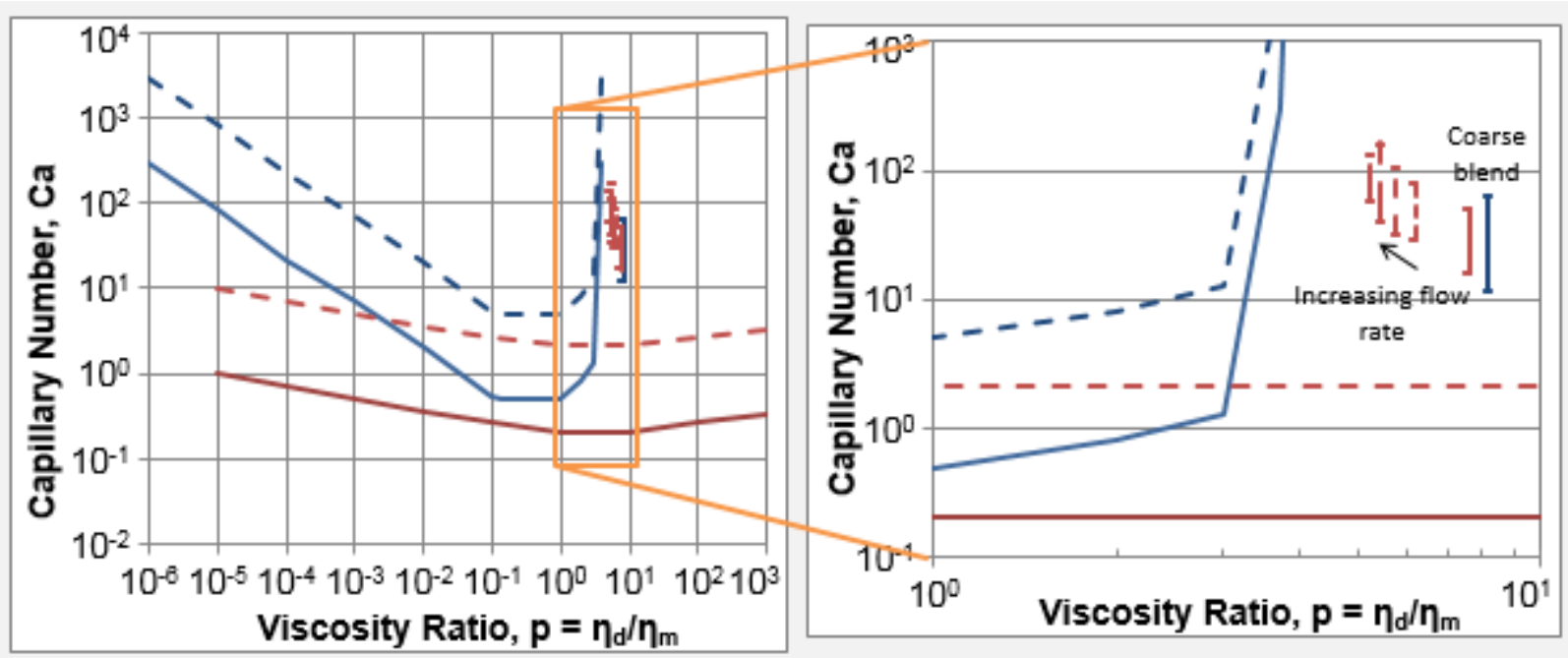

Figure 4-34: Effect of flow rate on Ca for converging cone dies.

The other important dimensionless numbers associated with extensional flow blending through the converging cone die with $0.330 \mathrm{~mm}$ exit diameter, $\varepsilon=7.1$, at various flow rates and at $220^{\circ} \mathrm{C}$ are given in Table $4-13$. Again, the extensional capillary numbers, $\mathrm{Ca}_{\mathrm{e}}$, for the various blends are much closer to $\mathrm{Ca}_{\text {crit }}$ than are the values for $\mathrm{Ca}_{\mathrm{s}}$. This makes sense because the converging cone dies with very short land lengths at the exit have flow fields dominated by uniaxial extensional flow. In fact, $\mathrm{Ca}_{\text {crit }}$ for uniaxial extensional flow is larger than that for planar extensional flow, and this difference may account for the disparity between $\mathrm{Ca}_{\mathrm{e}}$ and $\mathrm{Ca}_{\text {crit }}$. Though, the transient nature of the flow and the viscoelastic constitutive rheology, including potential extensional strain hardening behavior, are likely contributors to the difference between $\mathrm{Ca}_{\mathrm{e}}$ and $\mathrm{Ca}_{\text {crit. }}$. 
Table 4-13: Dimensionless numbers for various flow rates through converging cone die. The converging cone die with $0.330 \mathrm{~mm}$ exit diameter and $\varepsilon=7.1$ was used at $220^{\circ} \mathrm{C}$.

\begin{tabular}{|c|c|c|c|c|c|c|}
\hline $\begin{array}{l}\text { Piston speed } \\
(\mathrm{mm} / \mathrm{min})\end{array}$ & \multirow{2}{*}{$\begin{array}{l}\text { Coarse } \\
\text { blend }\end{array}$} & 10 & 50 & 100 & 200 & 300 \\
\hline $\begin{array}{l}\text { Flow rate } \\
\left(\mathrm{cm}^{3} / \mathrm{min}\right)\end{array}$ & & 1.7 & 8.3 & 16.5 & 33.0 & 49.5 \\
\hline $\begin{array}{l}\text { p using } \eta_{\text {power- }} \\
\text { law,wall }\end{array}$ & 7.1 & 5.3 & 4.8 & 4.5 & 4.3 & 4.2 \\
\hline $\begin{array}{l}\mathrm{Ca}_{\mathrm{s}} \text { using } \mathrm{d}_{\mathrm{n}} \& \\
\eta_{\text {power-law,wall and }} \\
\text { using } \mathrm{d}_{\mathrm{v}} \text { and } \eta_{0} \\
\left(\mathrm{C} \mathrm{a}_{\mathrm{s}} / \mathrm{C} \mathrm{a}_{\mathrm{crit}}\right)\end{array}$ & $\begin{array}{r}26-240 \\
(120-960)\end{array}$ & $\begin{array}{r}79-7,200 \\
(360- \\
29,000)\end{array}$ & $\begin{array}{r}130- \\
29,000 \\
(590- \\
110,000)\end{array}$ & $\begin{array}{r}135- \\
51,000 \\
(610- \\
210,000)\end{array}$ & $\begin{array}{r}170- \\
120,000 \\
(770- \\
500,000)\end{array}$ & $\begin{array}{r}300- \\
170,000 \\
(1,400- \\
670,000)\end{array}$ \\
\hline $\begin{array}{l}\text { Re, using } d_{v} \text { and } \\
\eta_{0} \text { and using } d_{n} \\
\text { and } \eta_{\text {power-law,wall }}\end{array}$ & - & $\begin{array}{r}5 \times 10^{-6}- \\
5 \times 10^{-4}\end{array}$ & $\begin{array}{r}3 \times 10^{-5}- \\
8 \times 10^{-3}\end{array}$ & $\begin{array}{r}7 \times 10^{-5}- \\
3 \times 10^{-2}\end{array}$ & $\begin{array}{r}1 \times 10^{-4}- \\
9 \times 10^{-2}\end{array}$ & $\begin{array}{r}2 \times 10^{-4}- \\
1 \times 10^{-1}\end{array}$ \\
\hline $\mathrm{t}_{\text {res }}(\mathrm{s})$ & - & 7.16 & 1.43 & 0.72 & 0.36 & 0.24 \\
\hline$\overline{\dot{\varepsilon}}\left(s^{-1}\right)$ & - & 1.0 & 5.0 & 10.0 & 19.9 & 29.9 \\
\hline $\begin{array}{l}C a_{e} \text {, using } d_{n} \text { and } \\
\text { using } d_{v} \text {, both } \\
\text { with } \eta_{e}=3 \eta_{0} \\
\left(C a_{e} / C a_{\text {crit }}\right)\end{array}$ & - & $\begin{array}{r}1-3 \\
(4-11)\end{array}$ & $\begin{array}{r}4-11 \\
(16-44)\end{array}$ & $\begin{array}{r}6-20 \\
(25-79)\end{array}$ & $\begin{array}{r}12-48 \\
(48-190)\end{array}$ & $\begin{array}{r}28-63 \\
(110-260)\end{array}$ \\
\hline $\mathrm{De}$ & - & 0.4 & 1.9 & 3.8 & 7.5 & 11.3 \\
\hline Wi & - & 2.7 & 13.4 & 26.9 & 53.8 & 80.7 \\
\hline
\end{tabular}

The full set of data for extensional flow blending of 5\% HDPE/PS through the converging cone dies is given in Figure 4-35, where both the extensional strain and flow rate were varied. The temperature was constant at $220^{\circ} \mathrm{C}$ as it was for all processing of $5 \%$ HDPE/PS. As can be seen, the effect of increasing flow rate was slightly different for the two dies with different extensional strain. For the $0.559 \mathrm{~mm}$ converging cone diameter, $\varepsilon=6.1$, die, $d_{\mathrm{v}}$ decreased to a stable level immediately and $d_{\mathrm{n}}$ was not affected by extensional flow blending. Therefore, P.I. likewise was constant with respect to flow rate. The log-normal mean, $\mu$, for the $0.559 \mathrm{~mm}(\varepsilon=$ 6.1) actually increased slightly from the coarse blend then stabilized, but the log-normal standard deviation, $\sigma$, decreased. The morphology after extensional flow blending through the $0.330 \mathrm{~mm}(\varepsilon=7.1)$ die reached the same $d_{v}$ as for the $0.559 \mathrm{~mm}$ die at a flow rate of 17 $\mathrm{cm}^{3} / \mathrm{min}$, but this die with greater extensional strain did affect $d_{\mathrm{n}}$, which was about half that of 
the die with smaller extensional strain. The P.I. for the $0.330 \mathrm{~mm}$ die was larger than that for the $0.559 \mathrm{~mm}$ die and stayed comparable to the P.I. of the coarse blend. Considering the lognormal-fitted data, the mean for the $0.330 \mathrm{~mm}$ die decreased by a factor of about 3.5 compared to the coarse blend and was a factor of about 4.7 smaller than the mean using the $0.559 \mathrm{~mm}$ die. The log-normal standard deviation increased slightly using the $0.330 \mathrm{~mm}$ die and was consistently larger than that using the $0.559 \mathrm{~mm}$ die. In summary, the use of a converging cone die with smaller extensional strain is useful in breaking up large drops in a blend and narrowing the size distribution of the morphology, while the use of a converging cone die with larger extensional strain is useful in breaking up both large and small drops in a blend, while maintaining a relatively stable size distribution, thereby shifting the entire morphology toward a finer dispersion.

The increased effect on overall dispersion using the converging cone die with greater extensional strain comes at the price of a larger pressure drop, as shown in Figure 4-36. Both converging cone dies show pressure drop that is linearly dependent on flow rate on a log-log plot, but the pressure drop for flow through the $0.330 \mathrm{~mm}(\varepsilon=7.1)$ is on average 3.3 times greater than that for flow through the $0.559 \mathrm{~mm}(\varepsilon=6.1)$. So, with processing at a flow rate of $17 \mathrm{~cm}^{3} / \mathrm{min}$, the die with $16 \%$ larger extensional strain produced a morphology with a $d_{\mathrm{n}}$ that was a factor of 2.3 smaller and a log-normal $\mu$ that was a factor of 5.2 smaller, but required a $\Delta P$ that was 2.6 times larger. 


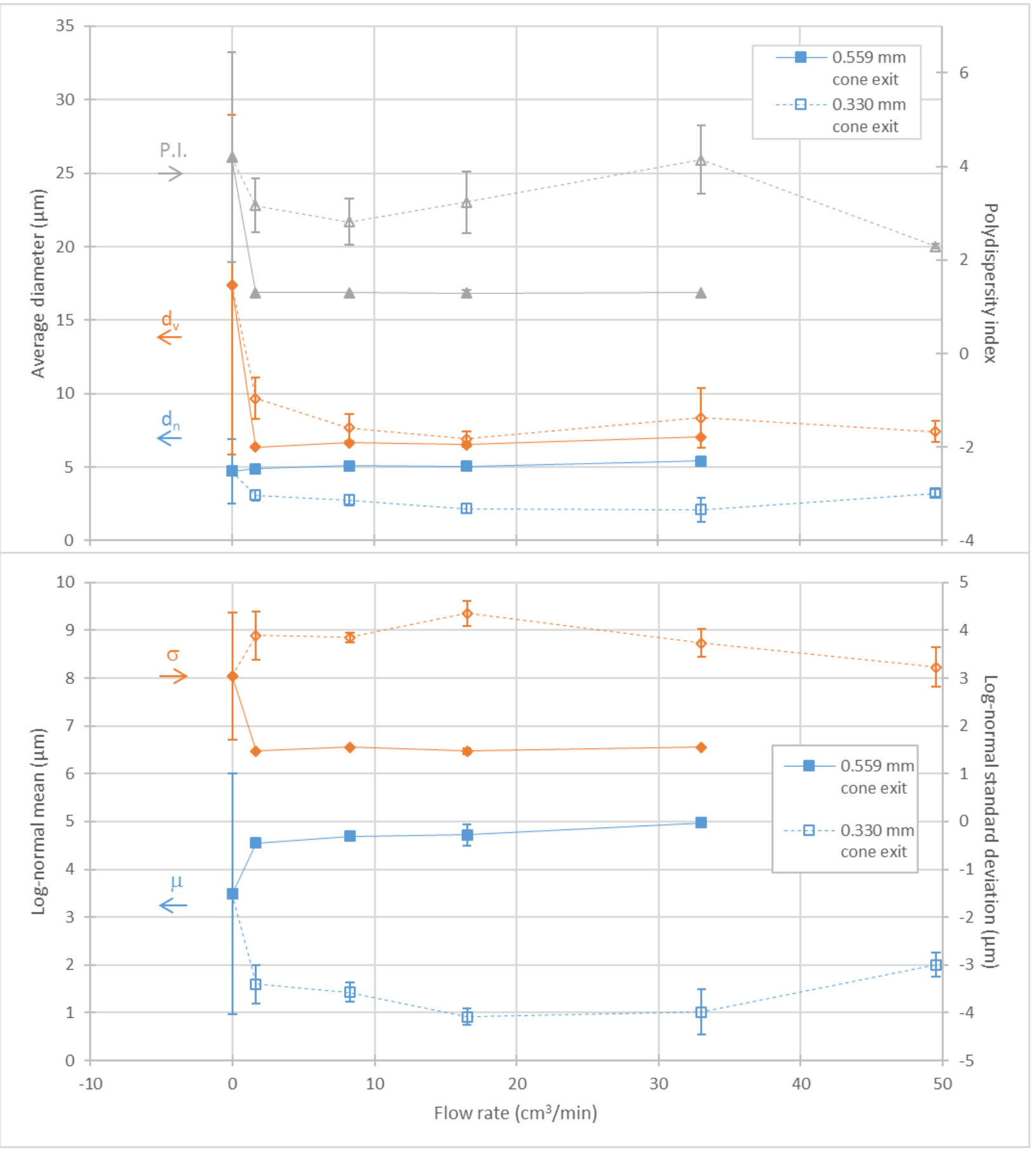

Figure 4-35: Morphology after extensional flow through converging cone dies. Top $-d_{\mathrm{n}}, d_{\mathrm{v}}$, and P.I. for extensional flow blending of 5\% HDPE/PS through the converging cone dies, varying both extensional strain and flow rate. Bottom - Mean and standard deviation from log-normal fits of the discrete histograms. Closed symbols with solid curves represent the blends after flow through the converging cone die with $0.559 \mathrm{~mm}$ exit diameter, $\varepsilon=6.1$, and open symbols with dotted curves represent those using the $0.330 \mathrm{~mm}$ exit diameter, $\varepsilon=7.1$. Error bars represent \pm 1 standard deviation across 3 replicate cross sections, when applicable. 


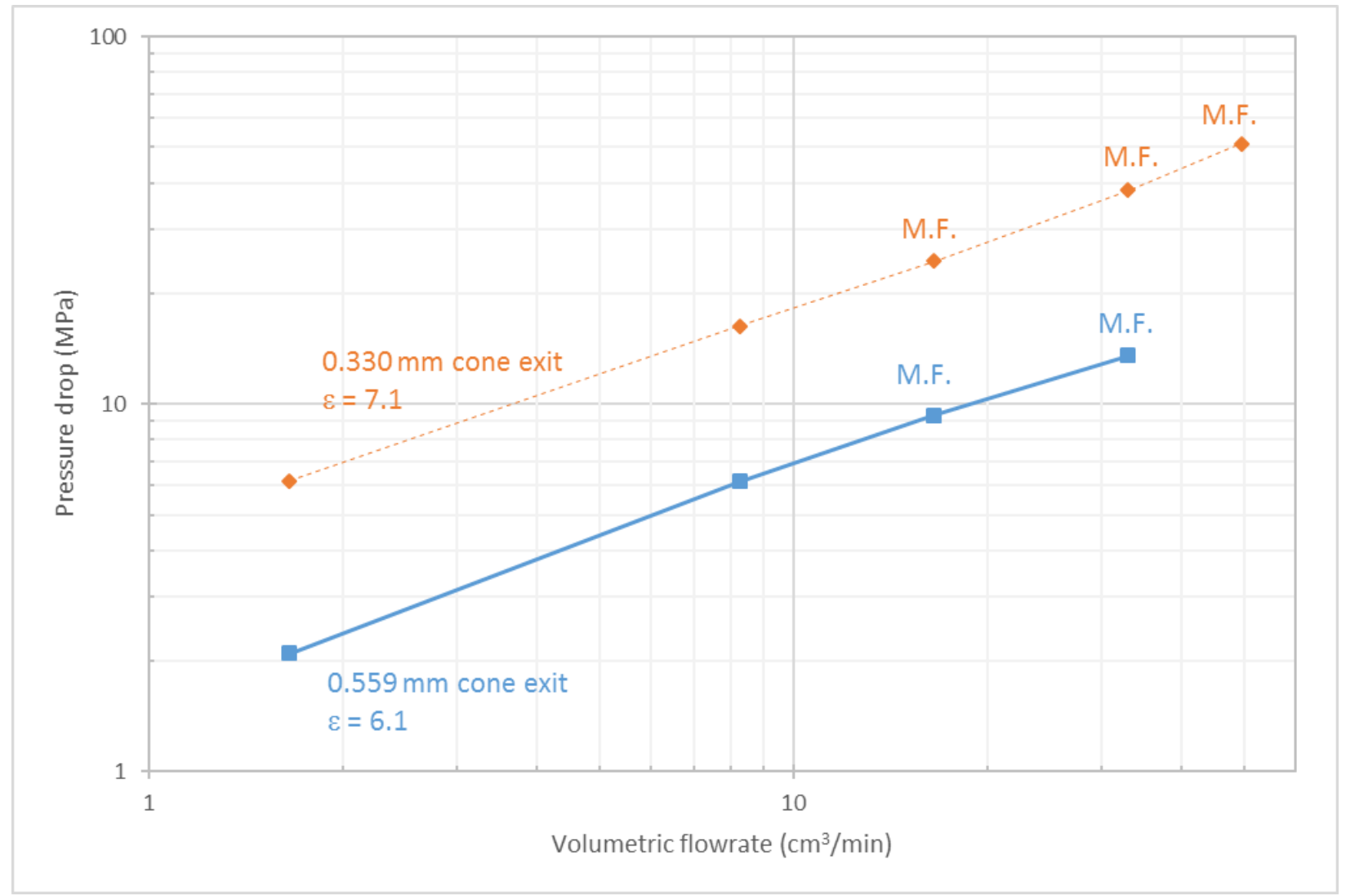

Figure 4-36: Pressure drop for extensional flow through converging cone dies

\subsubsection{Morphological Comparisons to Theory}

Several theories have been developed throughout the years describing the behavior of immiscible polymers during blending processes. These are described in Section 2.2. Summaries of the comparisons between the measured morphology and those predicted by various theories and models are given in Table 4-14 and Table 4-15 for the blends after extensional flow blending through the converging cone dies with $0.559 \mathrm{~mm}$ and $0.330 \mathrm{~mm}$ exit diameters, respectively. Taylor's theory [8] is the foundation for the study of the breakup of liquid threads dispersed in another liquid. The equation for the drop diameter is the following:

$$
d_{\text {Taylor }}=\frac{\sigma_{12}}{\eta_{\mathrm{m}} \dot{\gamma}}\left(\frac{16 p+16}{19 p+16}\right) .
$$

However, because the theory is only valid for isolated threads and Newtonian liquids, its predictions for the phase size are usually the lower limit for the expected phase size of non- 
isolated, viscoelastic blends. Likewise, another estimate of the lower limit may be calculated using $\mathrm{Ca}_{\text {crit, }}$ the critical limit for binary breakup of an isolated, initially spherical, Newtonian drop in a Newtonian matrix under quasi-steady state, or stepwise equilibrium, conditions. The following equation may be used to calculate the minimum drop size, $R_{\min }$, based on stepwise equilibrium breakup at $\mathrm{Ca}_{\text {crit }}[11]$ :

$$
R_{\text {min }}=2^{-1 / 3} \frac{\mathrm{Ca}_{\text {crit }} \sigma_{12}}{\eta_{\mathrm{m}} \dot{\gamma}} .
$$

Wu developed a semi-empirical relation to predict the dispersed-phase size for blends of $15 \%$ rubber in plastic, blended in co-rotating twin-screw extruders [72]:

$$
d_{\mathrm{Wu}}=4 p^{+0.84}\left(\frac{\sigma_{12}}{\eta_{\mathrm{m}} \dot{\gamma}}\right) .
$$

Because the model was developed with a twin-screw extruder and was specific to certain types of blends, Wu's model is not particularly useful for the processing of this work.

The last two theories to which the morphological results were compared are those described in Janssen's dissertation [11] for affine deformation of the dispersed phase, followed by breakup either in a downstream quiescent region or breakup during the flow. For this, first Ca was calculated using the zero-shear viscosity, $\eta_{0}$, and $\overline{\dot{\varepsilon}}$ and compared to $\mathrm{Ca}_{\text {crit. }}$ If $\mathrm{Ca} / \mathrm{Ca}_{\text {crit }}>$ 5 , then the dispersed phase undergoes affine deformation with the matrix phase. The width $B$, length $L$, and radius $R_{\text {affine }}$ of the thread are given by the following for affine deformation in planar extensional flow with interfacial tension maintaining a circular cross section [11]:

$$
\begin{gathered}
B=2 R_{0} \mathrm{e}^{-\varepsilon / 2}, \\
L=2 R_{0} \mathrm{e}^{\varepsilon}, \text { and } \\
R_{\text {affine }}=\frac{B}{2},
\end{gathered}
$$

where $R_{0}$ is the original drop radius, in this case, given by $d_{v} / 2$ of the coarse $5 \%$ HDPE/PS blend. For downstream breakup in a quiescent region, Tomotika's theory for breakup of a Newtonian thread in a Newtonian matrix due to sinusoidal disturbances on the surface of the liquid thread [14]. This theory yields the following:

$$
R_{\mathrm{drop}}=R_{\mathrm{affine}}\left(\frac{3 \pi}{2 x_{\mathrm{m}}}\right)^{1 / 3},
$$


where $x_{m}$ is the dominant wave number for the fatal surface disturbance that grows faster than all others and causes breakup of the thread, given in Figure 2-7. The drop size predicted by Tomotika's theory for breakup in a quiescent region is comparable to the measured $d_{\mathrm{n}}$ of the blends. It was $2.4 \mu \mathrm{m}$ for blending using the $0.559 \mathrm{~mm}$ converging cone die, a factor of 2.0 smaller than the typical measured $d_{\mathrm{n}}$ using that die, and it was $1.4 \mu \mathrm{m}$ for the $0.330 \mathrm{~mm}$ converging cone die, a factor of 1.5 smaller than the smallest $d_{\mathrm{n}}$ observed using that die.

Finally, Janssen's dissertation [11] also includes the theoretical approach to determining whether breakup of the elongated dispersed-phase domains will occur during flow, rather than in a downstream quiescent region. A dimensionless stretch rate is used as the independent variable for this theory:

$$
\text { Dimensionless stretch rate }=\frac{\eta_{\mathrm{m}} \overline{\dot{\varepsilon}} \alpha_{0}}{\sigma_{12}},
$$

where $\alpha_{0}$ is the initial surface disturbance amplitude due to Brownian motion, given in Equation (2-24), and almost always equal to about $1 \mathrm{~nm}$. Using this dimensionless stretch rate, the critical thread radius for breakup to occur during flow can be read from the plot in Figure 2-10. The following condition must be satisfied for breakup to occur during flow, otherwise the thindiameter threads are stable during flow and breakup downstream in a quiescent region:

$$
R_{\text {affine }} \leq R_{\text {crit }} \text {. }
$$

Relation (4-24) was not satisfied for flow through either of the converging cone dies at any of the flow rates, and therefore the predicted drop size, $d_{\text {drop, }}$ corresponding to breakup during flow and obtained by reading the plot in Figure 2-11, is not a valid prediction.

Therefore, the drop size after breakup in a quiescent downstream region, according to Tomotika's theory, is the best predictor considered in this work. However, the major shortcoming of this theoretical approach is that the sample collection used in the experimental methods of this work did not lend itself to downstream breakup. The extrudate was quenched in water soon after extruding from the bottom of the die, and the elongated threads of HDPE were likely frozen in place in the samples analyzed. Since the morphology analysis mainly considered only the cross section of the extrudate strands, it was not possible to distinguish dispersed-phase elongated threads from dispersed-phase quasi-spherical drops because both appear as circular domains in the planar cross section. Though, elongated domains were 
observed when the cross section was not truly orthogonal to the flow direction and when alternative sample preparation was taken, as shown in the selective dissolution experimental results given in Section 4.2.6.

Table 4-14: Morphology comparisons to theory for $0.559 \mathrm{~mm}$ converging cone die

\begin{tabular}{|c|c|c|c|c|c|c|}
\hline \multicolumn{2}{|c|}{ Converging cone exit diameter $(\mathrm{mm})$} & \multicolumn{5}{|c|}{0.559} \\
\hline \multirow{2}{*}{\multicolumn{2}{|c|}{ Piston speed $(\mathrm{mm} / \mathrm{min})$}} & \multicolumn{5}{|c|}{6.1} \\
\hline & & \multirow{2}{*}{$\begin{array}{l}\text { Coarse } \\
\text { blend }\end{array}$} & \multicolumn{2}{|r|}{50} & \multirow{2}{*}{$\frac{100}{17}$} & \multirow{2}{*}{$\frac{200}{33}$} \\
\hline & Flow rate $\left(\mathrm{cm}^{3} / \mathrm{min}\right)$ & & 2 & 8 & & \\
\hline \multirow{2}{*}{ Measured } & $d_{n}(\mu m)$ & 4.7 & 4.9 & 5.1 & 5.0 & 5.4 \\
\hline & $\mathrm{d}_{\mathrm{v}}(\mu \mathrm{m})$ & 17.4 & 6.4 & 6.6 & 6.5 & 7.0 \\
\hline \multirow{3}{*}{ Taylor } & $\mathrm{d}$, using $\eta_{0}(\mu \mathrm{m})$ & 0.0307 & 0.0028 & 0.0006 & 0.0003 & 0.0001 \\
\hline & $\begin{array}{l}d, \text { using } \eta_{\text {power-law,wall }} \\
(\mu \mathrm{m})\end{array}$ & 0.078 & 0.031 & 0.017 & 0.013 & 0.010 \\
\hline & $\begin{array}{l}\text { d, using } \bar{\varepsilon} \& \eta_{\mathrm{e}}=3 \eta_{0} \\
(\mu \mathrm{m})\end{array}$ & - & 1.7 & 0.3 & 0.2 & 0.1 \\
\hline \multirow{3}{*}{$\begin{array}{l}\text { Stepwise } \\
\text { equilibrium } \\
\text { minimum } \\
\text { using } \mathrm{Ca}_{\text {crit }}\end{array}$} & $\begin{array}{l}\text { d, using } \dot{\gamma}_{\text {app, wall } \&} \eta_{0} \\
(\mu \mathrm{m})\end{array}$ & 0.0144 & 0.00129 & 0.00026 & 0.00013 & 0.00006 \\
\hline & $\begin{array}{l}\mathrm{d} \text {, using } \dot{\gamma}_{\text {power-law,wall }} \& \\
\eta_{\text {power-law,wall }}(\mu \mathrm{m})\end{array}$ & 0.032 & 0.013 & 0.007 & 0.005 & 0.004 \\
\hline & $\begin{array}{l}\text { d, using } \bar{\varepsilon} \& \eta_{\mathrm{e}}=3 \eta_{0} \\
(\mu \mathrm{m})\end{array}$ & - & 0.812 & 0.162 & 0.081 & 0.041 \\
\hline \multirow{2}{*}{$\begin{array}{l}\text { Wu, empirical } \\
\text { model for } 15 \% \\
\text { blend in TSE }\end{array}$} & $\mathrm{d}$, using $\eta_{0}(\mu \mathrm{m})$ & 7.84 & 0.70 & 0.14 & 0.07 & 0.04 \\
\hline & $\begin{array}{l}d, \text { using } \eta_{\text {power-law,wall }} \\
(\mu \mathrm{m})\end{array}$ & 1.87 & 0.64 & 0.32 & 0.23 & 0.17 \\
\hline \multirow{6}{*}{$\begin{array}{l}\text { Affine } \\
\text { deformation } \\
\text { followed by } \\
\text { breakup in } \\
\text { quiescent } \\
\text { region } \\
\text { (Janssen, } \\
\text { Tomotika) }\end{array}$} & Ca, using $\eta_{0} \& \bar{\varepsilon}$ & - & 1.4 & 7.0 & 14 & 28 \\
\hline & $\begin{array}{l}\mathrm{Ca} / \mathrm{Ca}_{\text {crit }} \text { (> } 5 \text { yields } \\
\text { affine deformation) }\end{array}$ & - & 5.7 & 28 & 57 & 113 \\
\hline & $\mathrm{R}_{\text {affine }}(\mu \mathrm{m})$ & - & \multicolumn{4}{|c|}{0.42} \\
\hline & $\begin{array}{l}\text { Diameter, } B \text {, of } \\
\text { elongated thread }(\mu \mathrm{m})\end{array}$ & - & \multicolumn{4}{|c|}{0.83} \\
\hline & $\begin{array}{l}\text { L of elongated thread } \\
(\mu \mathrm{m})\end{array}$ & - & \multicolumn{4}{|c|}{7,600} \\
\hline & $\begin{array}{l}\mathrm{d}_{\text {drop, }} \text { Tomotika's } \\
\text { theory }(\mu \mathrm{m})\end{array}$ & - & \multicolumn{4}{|c|}{2.4} \\
\hline \multirow{3}{*}{$\begin{array}{l}\text { Affine } \\
\text { deformation } \\
\text { with breakup } \\
\text { during flow } \\
\text { (Janssen) }\end{array}$} & $\begin{array}{l}\text { Dimensionless stretch } \\
\text { rate, } \eta_{\mathrm{m}} \dot{\bar{\varepsilon}} \alpha_{0} / \gamma_{12}\end{array}$ & - & 0.0002 & 0.0008 & 0.0016 & 0.0033 \\
\hline & $\begin{array}{l}R_{\text {crit }} \text { (If } R_{\text {affine }} \leq R_{\text {crit, }} \\
\text { then breakup occurs } \\
\text { during flow) }(\mu \mathrm{m})\end{array}$ & - & 0.015 & 0.011 & 0.008 & 0.008 \\
\hline & $\begin{array}{l}\mathrm{d}_{\text {drop, }} \text { after breakup } \\
\text { during flow }(\mu \mathrm{m})\end{array}$ & - & $\theta .040$ & 0.030 & 0.021 & 0.020 \\
\hline
\end{tabular}


Table 4-15: Morphology comparisons to theory for $0.330 \mathrm{~mm}$ converging cone die

\begin{tabular}{|c|c|c|c|c|c|c|c|}
\hline \multicolumn{2}{|c|}{ Converging cone exit diameter $(\mathrm{mm})$} & \multicolumn{6}{|c|}{0.330} \\
\hline \multirow{2}{*}{\multicolumn{2}{|c|}{$\begin{array}{r}\varepsilon \\
\text { Piston speed }(\mathrm{mm} / \mathrm{min}) \\
\end{array}$}} & \multicolumn{6}{|c|}{7.1} \\
\hline & & \multirow{2}{*}{$\begin{array}{l}\text { Coarse } \\
\text { blend }\end{array}$} & \multirow{2}{*}{$\frac{10}{2}$} & \multirow{2}{*}{$\begin{array}{c}50 \\
8\end{array}$} & \multirow{2}{*}{$\begin{array}{c}100 \\
17 \\
\end{array}$} & \multirow{2}{*}{$\begin{array}{c}200 \\
33\end{array}$} & \multirow{2}{*}{$\begin{array}{c}300 \\
50\end{array}$} \\
\hline & Flow rate $\left(\mathrm{cm}^{3} / \mathrm{min}\right)$ & & & & & & \\
\hline \multirow{2}{*}{ Measured } & $d_{n}(\mu m)$ & 4.7 & 3.1 & 2.8 & 2.2 & 2.1 & 3.2 \\
\hline & $\mathrm{d}_{\mathrm{v}}(\mu \mathrm{m})$ & 17.4 & 9.7 & 7.7 & 6.9 & 8.4 & 7.4 \\
\hline \multirow{3}{*}{ Taylor } & $d$, using $\eta_{0}(\mu \mathrm{m})$ & $3.1 \mathrm{E}-2$ & $5.7 \mathrm{E}-4$ & $1.1 \mathrm{E}-4$ & $5.7 \mathrm{E}-5$ & $2.8 \mathrm{E}-5$ & $1.9 \mathrm{E}-5$ \\
\hline & $\begin{array}{l}d, \text { using } \eta_{\text {power-law,wall }} \\
(\mu \mathrm{m})\end{array}$ & 0.0780 & 0.0169 & 0.0092 & 0.0070 & 0.0054 & 0.0046 \\
\hline & $\begin{array}{l}\text { d, using } \bar{\varepsilon} \& \eta_{\mathrm{e}}=3 \eta_{0} \\
(\mu \mathrm{m})\end{array}$ & - & 1.48 & 0.30 & 0.15 & 0.07 & 0.05 \\
\hline \multirow{3}{*}{$\begin{array}{l}\text { Stepwise } \\
\text { equilibrium } \\
\text { minimum } \\
\text { using } \mathrm{Ca}_{\text {crit }}\end{array}$} & $\begin{array}{l}\text { d, using } \dot{\gamma}_{\text {app, wall } \&} \eta_{0} \\
(\mu \mathrm{m})\end{array}$ & $7.2 \mathrm{E}-3$ & $1.3 \mathrm{E}-4$ & 2.7E-5 & $1.3 \mathrm{E}-5$ & $6.6 \mathrm{E}-6$ & $4.4 \mathrm{E}-6$ \\
\hline & $\begin{array}{l}d, \text { using } \dot{\gamma}_{\text {power-law,wall }} \& \\
\eta_{\text {power-law,wall }}(\mu \mathrm{m})\end{array}$ & 0.0179 & 0.0039 & 0.0021 & 0.0016 & 0.0012 & 0.0010 \\
\hline & $\begin{array}{l}\text { d, using } \bar{\varepsilon} \& \eta_{\mathrm{e}}=3 \eta_{0} \\
(\mu \mathrm{m})\end{array}$ & - & 0.692 & 0.138 & 0.069 & 0.035 & 0.023 \\
\hline \multirow{2}{*}{$\begin{array}{l}\text { Wu, empirical } \\
\text { model for } \\
15 \% \text { blend in } \\
\text { TSE }\end{array}$} & $d$, using $\eta_{0}(\mu \mathrm{m})$ & 7.840 & 0.145 & 0.029 & 0.014 & 0.007 & 0.005 \\
\hline & $\begin{array}{l}d, \text { using } \eta_{\text {power-law,wall }} \\
(\mu \mathrm{m})\end{array}$ & 1.87 & 0.32 & 0.16 & 0.12 & 0.08 & 0.07 \\
\hline \multirow{6}{*}{$\begin{array}{l}\text { Affine } \\
\text { deformation } \\
\text { followed by } \\
\text { breakup in } \\
\text { quiescent } \\
\text { region } \\
\text { (Janssen, } \\
\text { Tomotika) }\end{array}$} & Ca, using $\eta_{0} \& \dot{\varepsilon}$ & - & 2 & 8 & 17 & 33 & 50 \\
\hline & $\mathrm{Ca} / \mathrm{Ca}_{\text {crit }}$ & - & 7 & 33 & 66 & 130 & 200 \\
\hline & $R_{\text {affine }}(\mu \mathrm{m})$ & - & \multicolumn{5}{|c|}{0.25} \\
\hline & $\begin{array}{l}\text { Diameter, } B \text {, of } \\
\text { elongated thread }(\mu \mathrm{m})\end{array}$ & - & \multicolumn{5}{|c|}{0.49} \\
\hline & $\begin{array}{l}\text { L of elongated thread } \\
(\mu \mathrm{m})\end{array}$ & - & \multicolumn{5}{|c|}{21,900} \\
\hline & $\begin{array}{l}d_{\text {drop}}, \text { Tomotika's } \\
\text { theory }(\mu \mathrm{m})\end{array}$ & - & \multicolumn{5}{|c|}{1.4} \\
\hline \multirow{3}{*}{$\begin{array}{l}\text { Affine } \\
\text { deformation } \\
\text { with breakup } \\
\text { during flow, } \\
\text { Janssen }\end{array}$} & $\begin{array}{l}\text { Dimensionless stretch } \\
\text { rate, } \eta_{\mathrm{m}} \dot{\bar{\varepsilon}} \alpha_{0} / \gamma_{12}\end{array}$ & - & 0.0002 & 0.0010 & 0.0019 & 0.0038 & 0.0057 \\
\hline & $\begin{array}{l}R_{\text {crit }} \text { (If } R_{\text {affine }} \leq R_{\text {crit, }} \\
\text { then breakup occurs } \\
\text { during flow) }(\mu \mathrm{m})\end{array}$ & - & 0.015 & 0.010 & 0.008 & 0.008 & 0.008 \\
\hline & $\begin{array}{l}d_{\text {drop, }} \text { after breakup } \\
\text { during flow }(\mu \mathrm{m})\end{array}$ & - & $\theta .040$ & 0.030 & 0.021 & $\theta .020$ & 0.020 \\
\hline
\end{tabular}




\subsubsection{Orifice Dies}

In flow through the orifice dies, the available cross-sectional area for flow abruptly converges from the diameter of the capillary rheometer barrel (reservoir) to a small orifice diameter; this is referred to as a $180^{\circ}$ cone inlet. The tube length at the orifice diameter is minimized to avoid shear flow contributions to droplet dynamics and pressure drop. The orifice dies used in this work have $0.559 \mathrm{~mm}$ and $0.330 \mathrm{~mm}$ orifice diameters, respectively, and they may be used singly or in a stacked configuration, with the larger orifice-diameter die is stacked on top of the smaller orifice-diameter die, as described in Section 3.3.2.2.

The effects of extensional strain, stacking the orifice dies, and flow rate on the morphology of 5\% HDPE/PS are shown in Figure 4-37. After flow through the orifice dies, $d_{v}$ significantly decreased, regardless of geometry factors or flow rate, whereas $d_{\mathrm{n}}$ required a flow rate of $17 \mathrm{~cm}^{3} / \mathrm{min}$ or greater to yield a significant decrease. The size distribution dropped for lower flowrates according to the decrease in $d_{\mathrm{v}}$ and was slightly higher for higher flowrates with the further decrease in $d_{\mathrm{n}}$.

While Figure 4-37 shows the standard morphology descriptors used in this work, $d_{\mathrm{n}}, d_{\mathrm{v}}$, and P.I., Figure 4-38 shows the morphology as described by the mean, $\mu$, and standard deviation, $\sigma$, of a log-normal fit of the drop size distribution. Using these descriptors, the difference in effects of the different orifice die geometries is elucidated with the $0.330 \mathrm{~mm}$ orifice die distinguishing itself from the $0.559 \mathrm{~mm}$ orifice and the stacked orifice configuration. The $0.330 \mathrm{~mm}$ orifice die appears optimal to avoid any increase in the log-normal mean with extensional flow blending, though the other two die configurations offer the ability to control the size distribution, as given by the log-normal standard deviation, if desired. The hypothesis explaining why the stacked orifice dies yielded results more similar to those of the $0.559 \mathrm{~mm}$ orifice rather than the $0.330 \mathrm{~mm}$ orifice is that the stacked configuration contains a stagnant region between the two stretching episodes where coalescence may occur, negating positive effects that two stretching episodes would otherwise likely offer. 


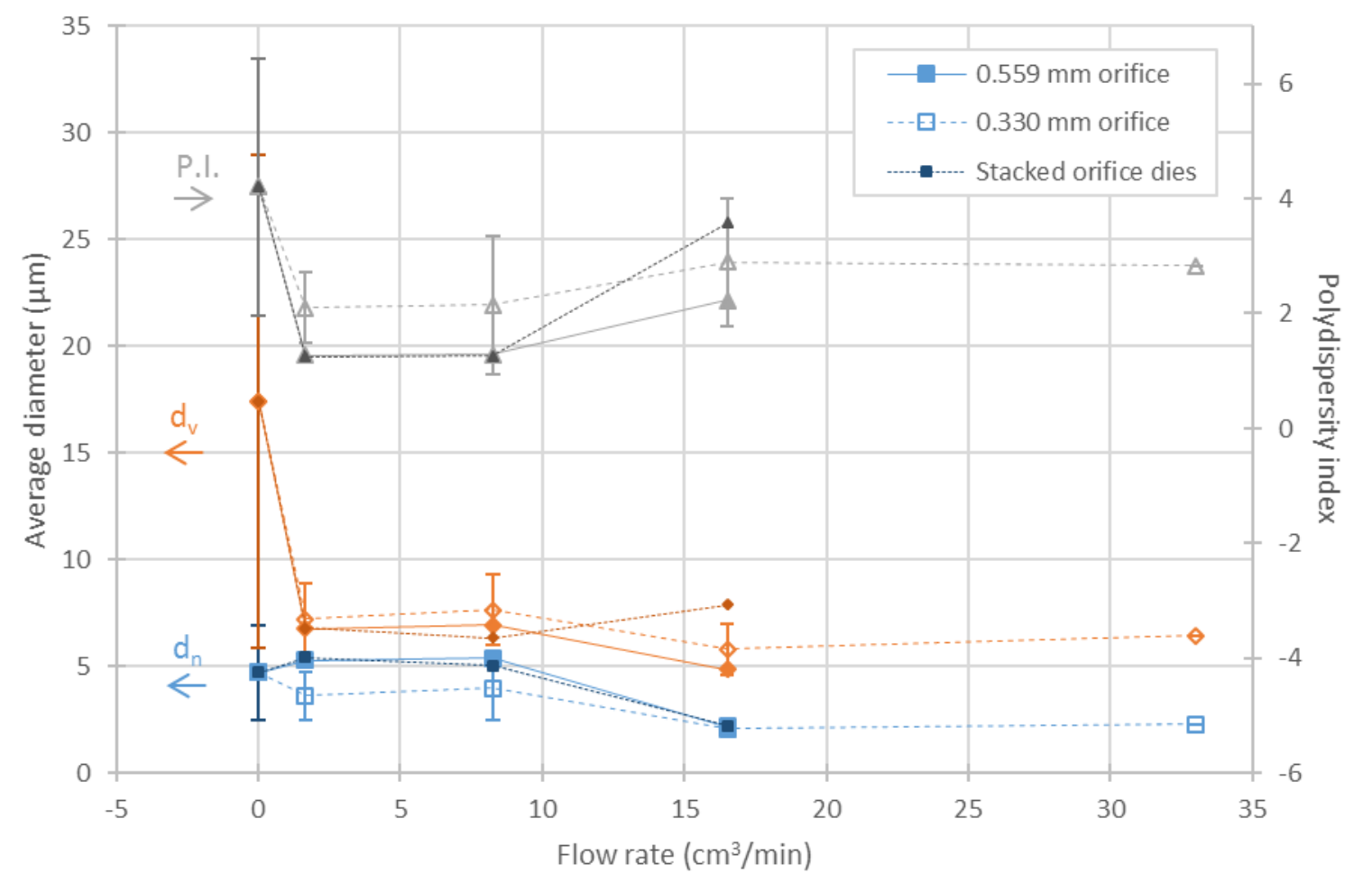

Figure 4-37: 5\% HDPE/PS morphology after flow through orifice dies. $d_{\mathrm{n}}, d_{\mathrm{v}}$, and P.I. morphology descriptors after flow through $0.559 \mathrm{~mm}$ orifice, $0.330 \mathrm{~mm}$ orifice, and stacked orifice dies at various flow rates and at $220^{\circ} \mathrm{C}$. Error bars $= \pm 1$ standard deviation across 3 replicate cross sections when applicable. 


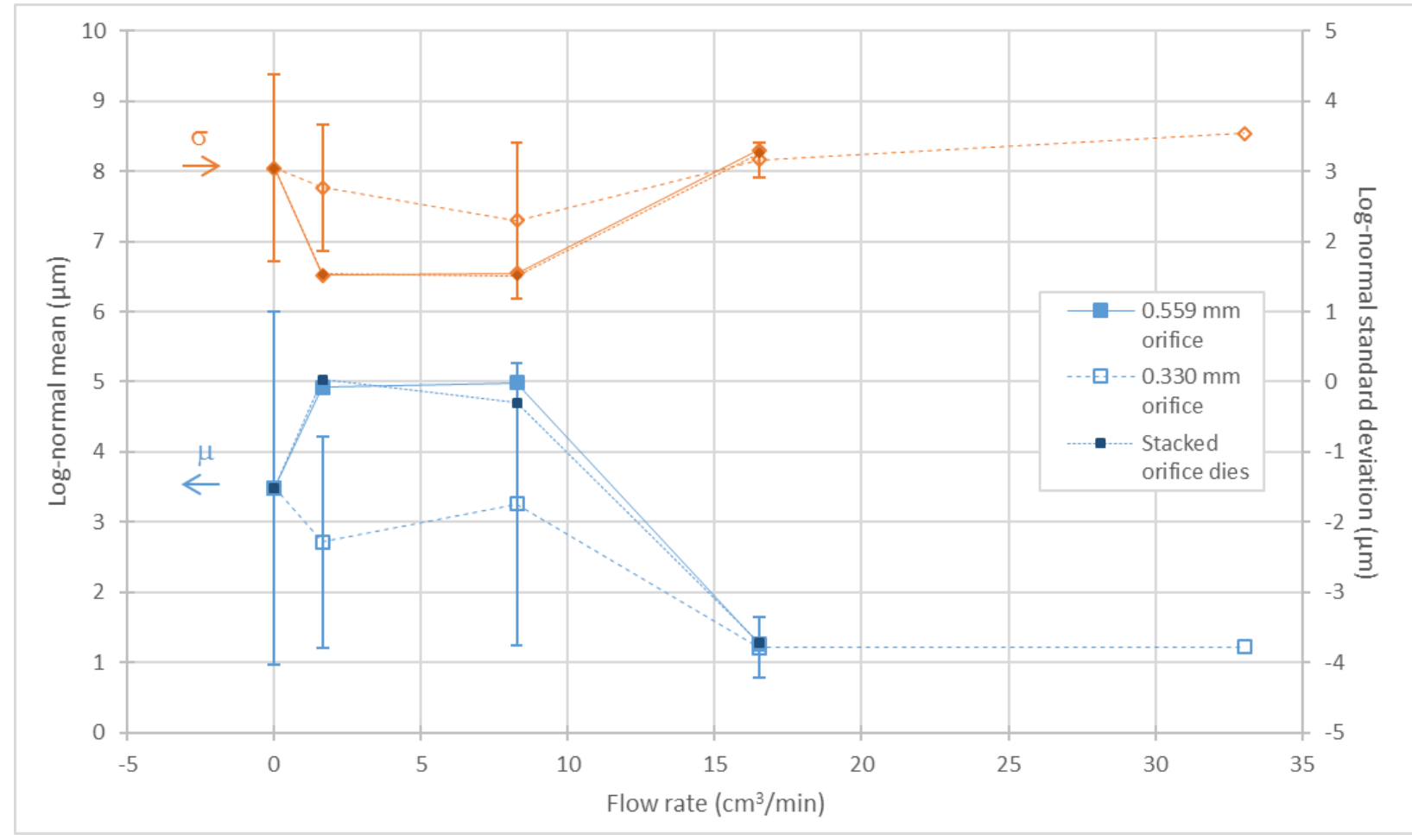

Figure 4-38: Log-normal morphology after flow through orifice dies. Lognormal mean $\mu$ and standard deviation $\sigma$ of dispersed phase with flow through orifice die with $0.559 \mathrm{~mm}$ diameter, $0.330 \mathrm{~mm}$ diameter, or stacked orifice dies at various flow rates and at $220^{\circ} \mathrm{C}$. Error bars $= \pm 1$ standard deviation across 3 replicate cross sections when applicable.

The pressure drop and the presence of melt fracture for flow through the orifice dies are given in Figure 4-39. The pressure drop for the most part scales linearly with flow rate on a log-log plot, which is to be expected for exit flows from a reservoir, through a convergence. As should be expected as well, the $0.330 \mathrm{~mm}$ orifice with $\varepsilon=7.6$ requires a higher pressure drop than does the $0.559 \mathrm{~mm}$ orifice with $\varepsilon=6.5$. Furthermore, the stacked configuration with $\varepsilon=$ $6.5 \& 6.0$ requires slightly higher pressure drop than does the $0.330 \mathrm{~mm}$ orifice. The $0.330 \mathrm{~mm}$ orifice was the only one to yield melt fracture of the extrudate strand as well. This occurs due to slippage at the wall due to fluid elasticity among other factors, and its onset typically occurs at a shear stress at the wall, $\tau_{\mathrm{w}}$, of about $10^{5} \mathrm{~Pa}$. Generally, melt fracture is avoided in industry because of the difficulties controlling the flow and the process. For all of these reasons, and since the $0.330 \mathrm{~mm}$ orifice die did not perform exceptionally better than the other orifice die 
configurations, the $0.559 \mathrm{~mm}$ orifice die is likely the most advantageous choice among the orifice dies for its effects on morphology compared to the pressure drop it requires.

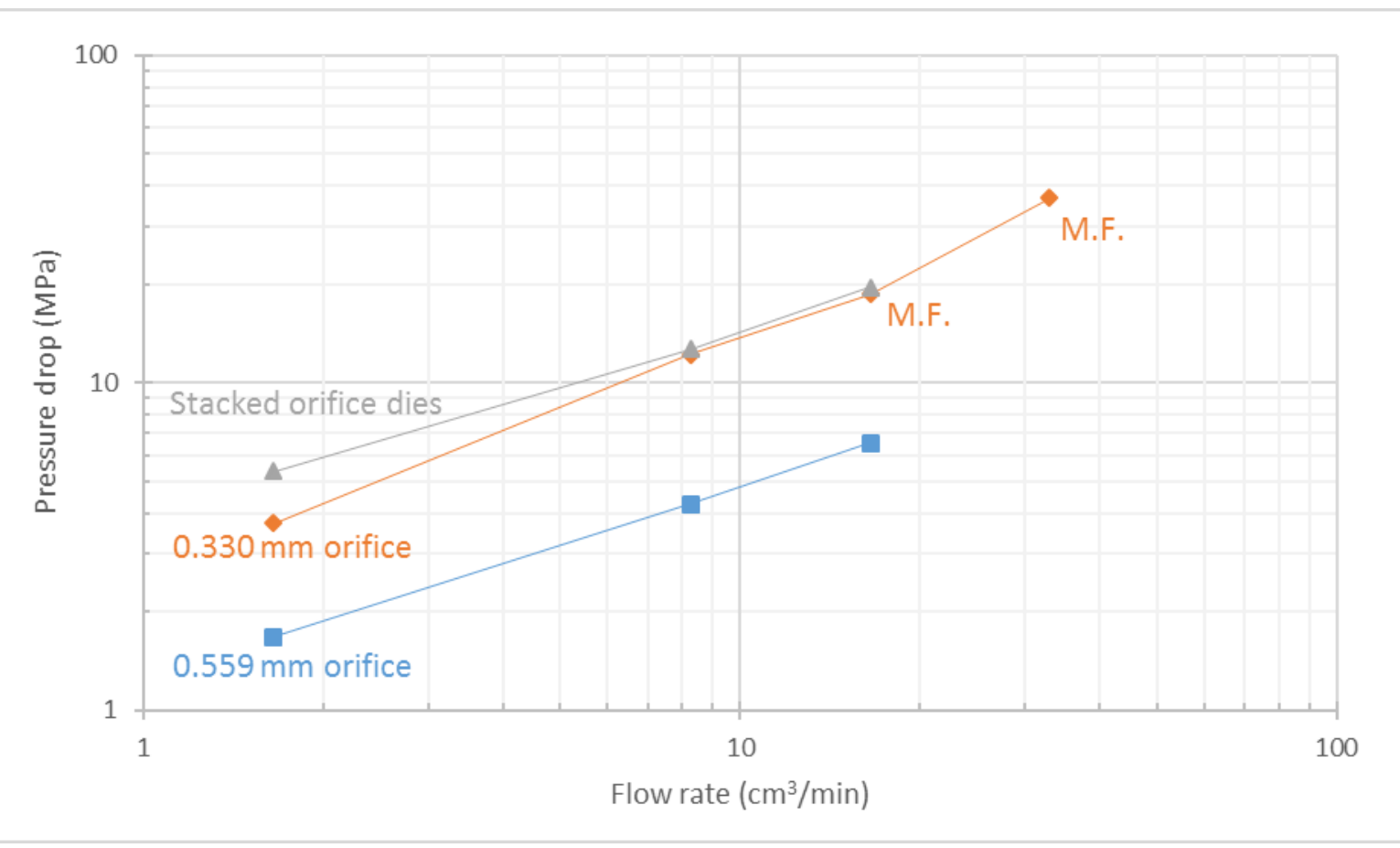

Figure 4-39: Pressure drop and melt fracture for flow through orifice dies. 5\% HDPE/PS through orifice dies at $220^{\circ} \mathrm{C}$. "M.F." denotes melt fracture, either on the surface of the extrudate strand or gross melt fracture.

\subsubsection{Disk-in-Tube Die}

The remainder of the converging-geometry dies investigated in this work were comprised of specially fabricated inserts that nested within the converging cone die with 0.559 $\mathrm{mm}$ exit diameter. The inserts were designed to impart one or more extra stretching episodes onto the blend in a single pass through the extensional flow cell (EFC). This was expected to increase the total extensional stresses imparted onto the dispersed phase and to add a brief quiescent region between the stretching episodes, where the flow fields relax and allow the dispersed phase domains to relax and potentially breakup. Furthermore, due to the designs chosen for the inserts, the first stretching episode consisted of large contributions of planar 
extensional flow, as well as uniaxial extensional and shear flows. Planar extensional flow is more efficient for dispersion than uniaxial extensional flow, so the first stretching episode of the dies incorporating inserts was expected to be the primary one to promote drop stretching and breakup.

The first of the dies with an insert was the disk-in-tube die, described in Section 3.3.2.3. This die consisted of an insert with a straight-walled disk that nested within the straight-walled round-cross section tube upstream of the converging cone region of the die. The gap between the disk insert and the tube wall was designed to be as tight as possible using the equipment available in the Chemical and Biomedical Engineering Machine Shop and was estimated to be about 0.001 in $(25.4 \mu \mathrm{m})$. Using this estimate of the annular gap, the extensional strain for the first stretching episode was $\varepsilon_{1}=4.5$, and the second stretching episode was that of the parent die, the converging cone die with $0.559 \mathrm{~mm}$ exit diameter and $\varepsilon_{2}=6.1$. The maximum apparent shear rate at the wall in the annular gap region was approximated by the equation for flow through a slit:

$$
\dot{\gamma}_{\text {app,wall }}=\frac{6 Q}{W H^{2}}=\frac{6 Q}{C G^{2}},
$$

where the average circumference $C$ between the disk and tube walls approximated the width $W$ of the slit and the gap $G$ between the disk and the tube wall approximated the height $H$ of the slit. Using Equation (4-25), the shear rate through the annular gap is given in Table 4-16. Also shown are the residence time, $t_{\text {res }}=V_{\text {annulus }} / Q$, and an approximation of the average extensional strain rate, where the entry convergence domain is approximated to be equal to that in the annular region. As can be seen, very large shear rates and extensional strain rates are predicted in the first stretching episode of the disk-in-tube die. 
Table 4-16: Flow field characteristics for disk-in-tube die

\begin{tabular}{|r|r|r|r|r|}
\hline $\begin{array}{c}\text { Piston } \\
\text { speed } \\
(\mathrm{mm} / \mathrm{min})\end{array}$ & $\begin{array}{c}\text { Q } \\
\left(\mathrm{cm}^{3} / \mathrm{min}\right)\end{array}$ & \multicolumn{1}{c|}{$\mathrm{t}_{\text {res }}(\mathrm{s})$} & $\dot{\gamma}_{\text {app,wall }}\left(\mathrm{s}^{-1}\right)$ & \multicolumn{1}{c|}{$\bar{\varepsilon}_{1}\left(\mathrm{~s}^{-1}\right)$} \\
\hline 10 & 2 & 0.118 & 6,980 & 38 \\
\hline 50 & 8 & 0.024 & 34,900 & 190 \\
\hline 100 & 17 & 0.012 & 69,800 & 381 \\
\hline 200 & 33 & 0.006 & 140,000 & 761 \\
\hline 300 & 50 & 0.004 & 209,000 & 1,140 \\
\hline
\end{tabular}

The effects of extensional flow blending through the disk-in-tube die on the morphology of $5 \% \mathrm{HDPE} / \mathrm{PS}$ at $220^{\circ} \mathrm{C}$ and various flow rates are shown in Figure 4-40. Flow through the disk-in-tube slightly decreased both $d_{\mathrm{n}}$ and $d_{\mathrm{v}}$ without a significant effect of flow rate. Likewise, the log-mean $\mu$ decreased. The size distribution, however, remained about the same as that of the coarse blend, as given by both P.I. and log-mean $\sigma$. The die required a considerably larger pressure drop than the converging cone and orifice dies, as given in Figure 4-41, and this limited the maximum flow rate achievable through the die to just $50 \mathrm{~mm} / \mathrm{min}$ piston speed, or 8 $\mathrm{cm}^{3} / \mathrm{min}$. The large pressure drop is likely due to the large shear contributions in the 0.001 in gap section between the disk and the tube wall, which is maintained over a length of about 3.5 $\mathrm{mm}$. 


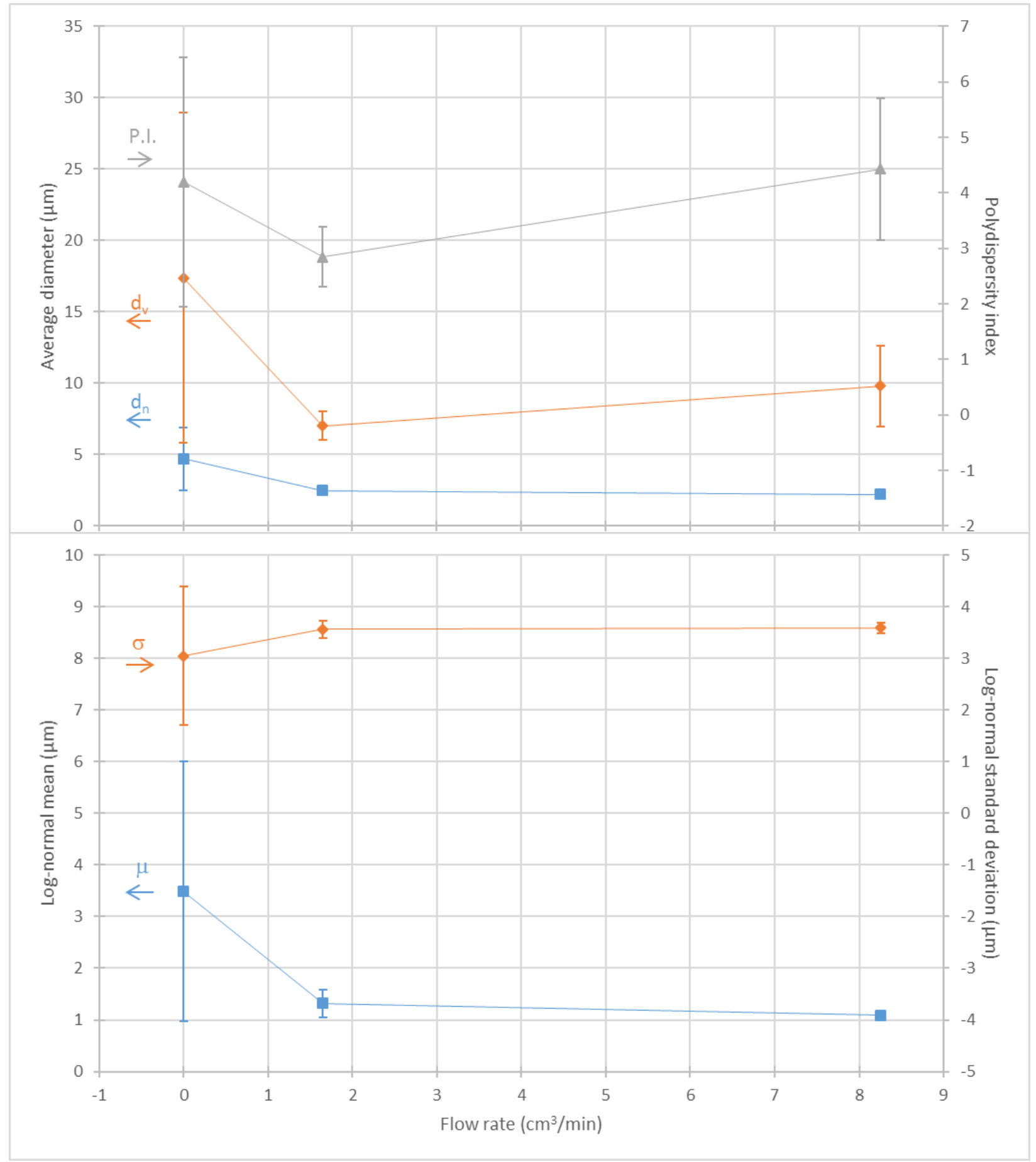

Figure 4-40: Effects of flow through disk-in-tube die on morphology. Top $-d_{n}, d_{v}$, and P.I. for extensional flow blending of 5\% HDPE/PS through the disk-in-tube die with increasing flow rate. Bottom - Mean and standard deviation from log-normal fits of the discrete histograms. Error bars represent \pm 1 standard deviation across 3 replicate cross sections, when applicable. 


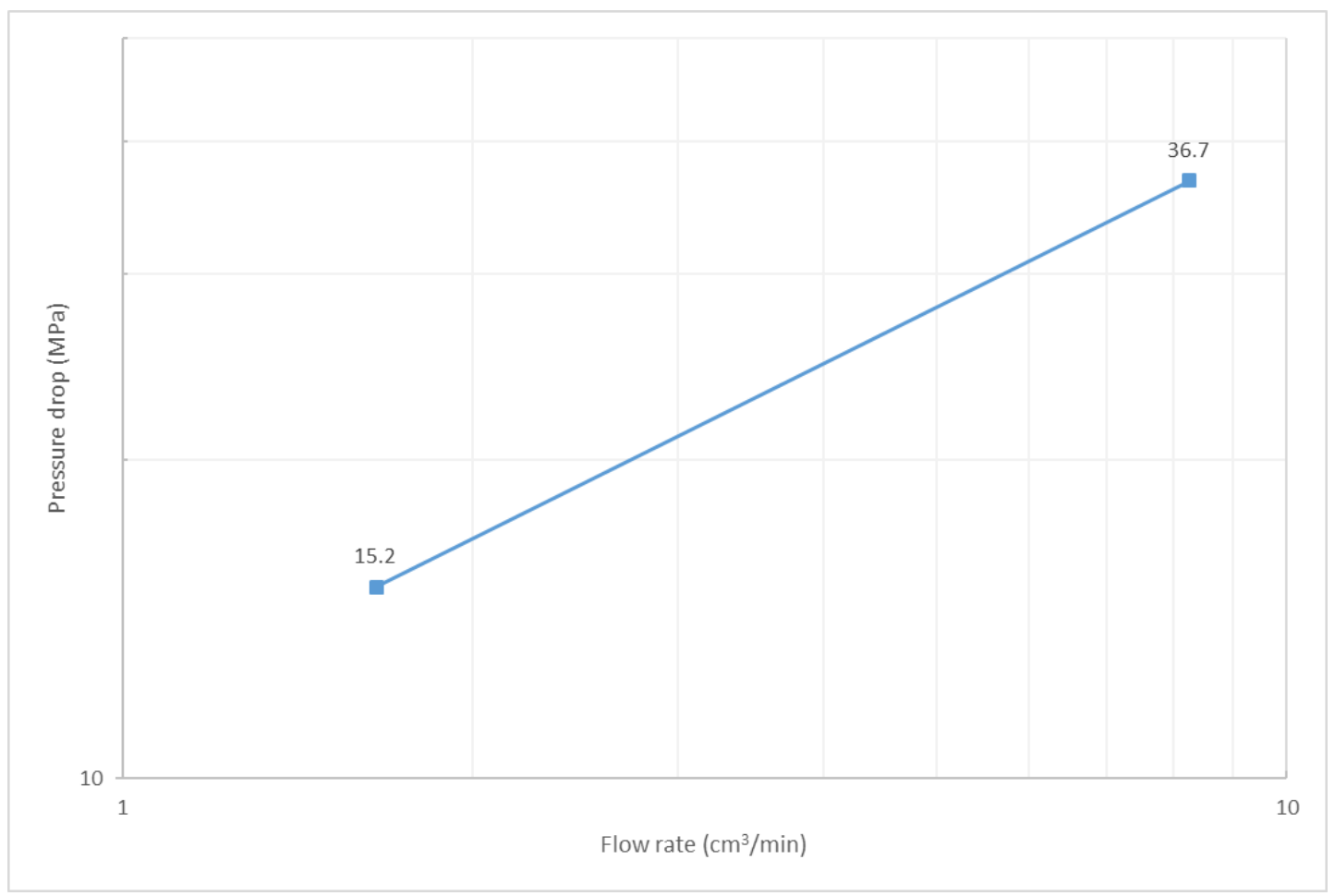

Figure 4-41: Pressure drop for flow through disk-in-tube die

\subsubsection{Hemisphere-in-Cone Dies}

Another fabricated die incorporating an insert was the hemisphere-in-cone die, described in Section 3.3.2.4. This die consisted of an insert with a rounded, or hemispherical, end that nested within the converging region of the $0.559 \mathrm{~mm}$ exit-diameter converging cone die. The gap between the hemisphere insert and the converging cone wall could be set by adding spacers, in the form of annular sheet metal shims, between the top of the insert and the shoulder on which is rested. The minimum area for flow through the first stretching episode was the conical frustum between the hemisphere and the converging cone wall, and the second stretching episode was at the exit of the $0.559 \mathrm{~mm}$ converging cone die. The extensional strains associated with each of the two stretching episodes, dependent on the spacer thickness used, are given in Table 3-5 and in Figure 4-42. The first stretching episode's $\varepsilon_{1}$ varied from 2.9 to 5.7 using spacers 0.016 in to 0.001 in (406 to $25.4 \mu \mathrm{m}$ ) thick, and the second stretching episode's $\varepsilon_{2}$ was relatively constant at about 3.8. The average extensional strain rates through 
both of the stretching episodes with various spacers were also calculated. The volume of the converging region entering each stretching episode was calculated and divided by the volumetric flow rate to yield the average residence time for flow through each convergence. Then the extensional strain through each stretching episode was divided by the residence time to yield the average extensional strain rates, which are presented in Figure 4-43. The second stretching episode yielded very high extensional strain rates because the residence time in the converging section between the hemisphere insert and the converging cone exit was very short.

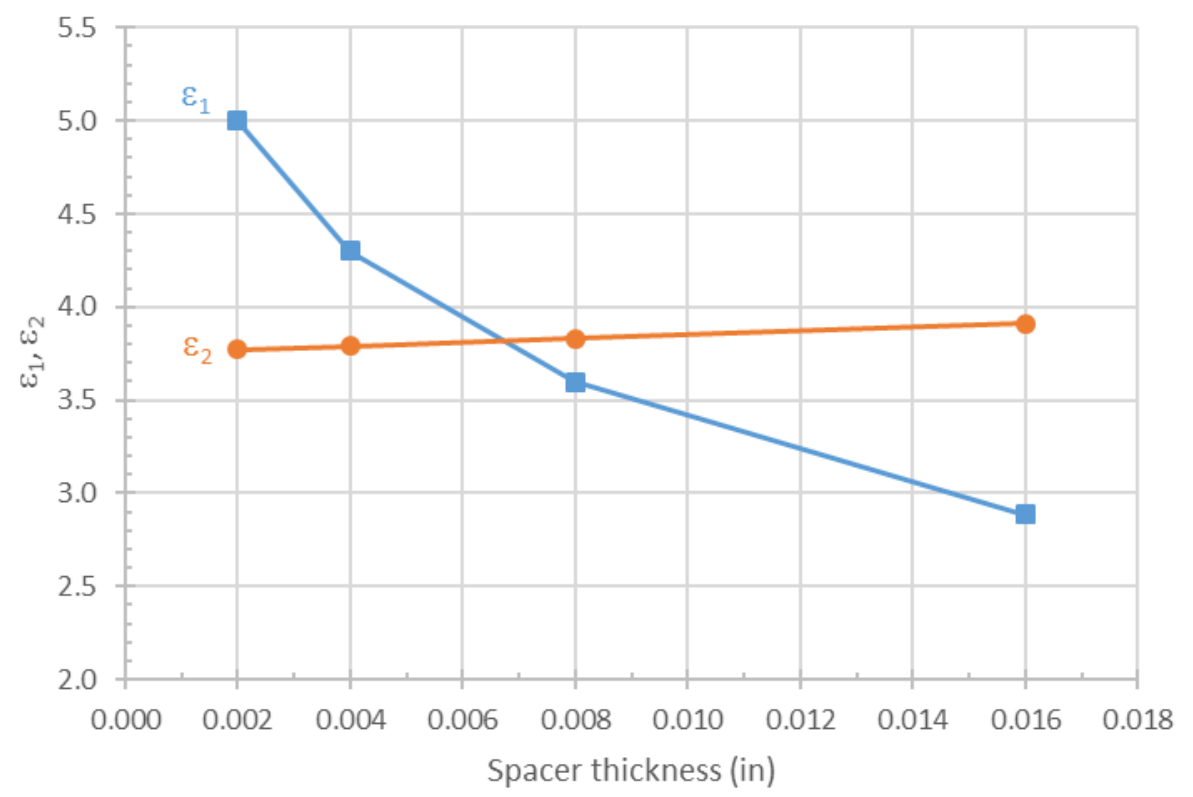

Figure 4-42: Extensional strains through hemisphere-in-cone die 


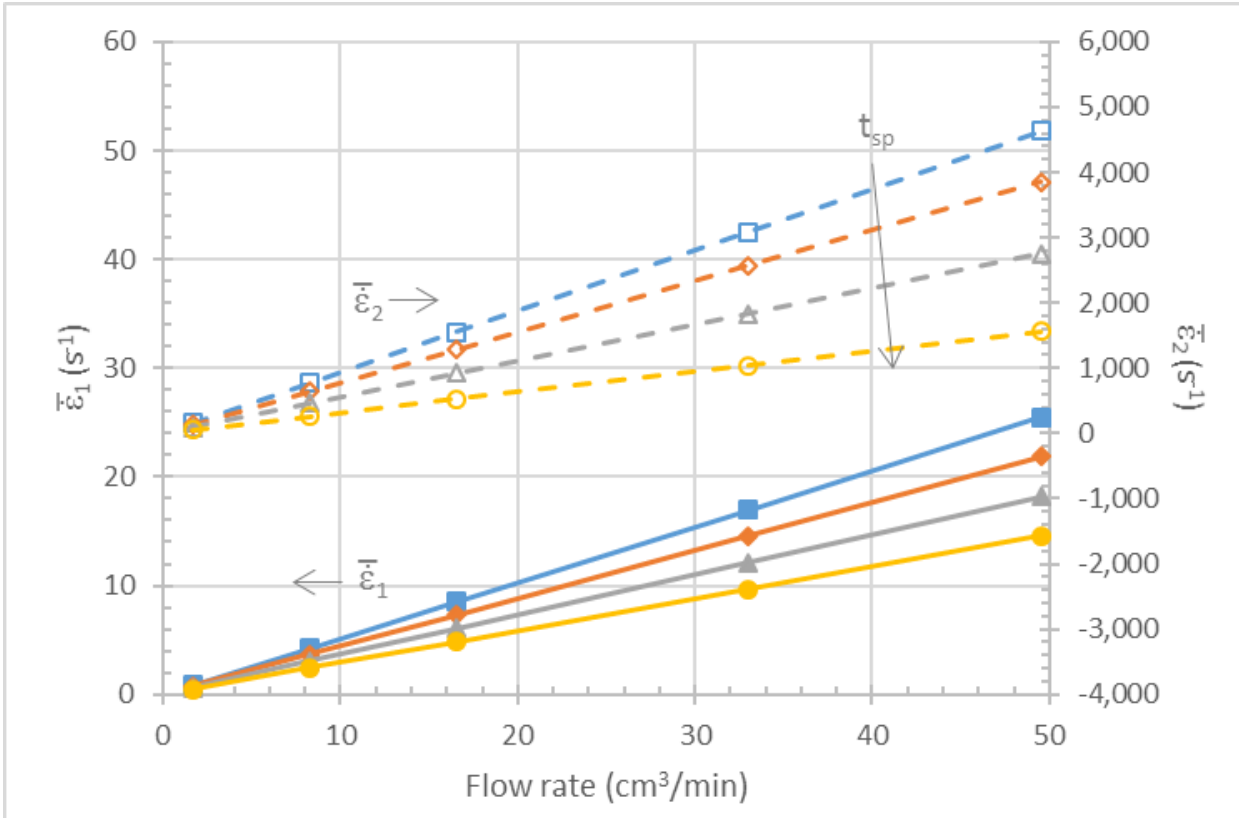

Figure 4-43: Average extensional strain rates through hemisphere-in-cone die

The results of extensional flow blending of the 5\% HDPE/PS blend using the hemispherein-cone die on morphology are given in Figure 4-44. Processing using the hemisphere-in-cone die with spacers ranging from 0.002 in to 0.016 in decreased both $d_{\mathrm{n}}$ and $d_{\mathrm{v}}$; however, the trend with respect to decreasing spacer thickness and thereby the gap setting of the convergence of the first stretching episode was not always consistent. The trend at a piston speed of 100 $\mathrm{mm} / \mathrm{min}$, which is $17 \mathrm{~cm}^{3} / \mathrm{min}$, did show an inversely proportional relationship between the log-normal $\mu$ and the spacer thickness, as would be expected. Three of the four configurations proved adept at decreasing the phase size of the blend, except the smallest spacer, 0.002 in $(0.051 \mathrm{~mm})$, for which only the slowest flow rate was achievable. The pressure drop and piston force remained lower than the rheometer's limits, but the melt flow never reached an equilibrium at larger flowrates. One hypothesis is that gaps too small are prone to clogging by particulates and micro-scale degraded components in the blend. A spacer shim of 0.001 in $(0.025 \mathrm{~mm})$ was also investigated but did not yield flow at all. 


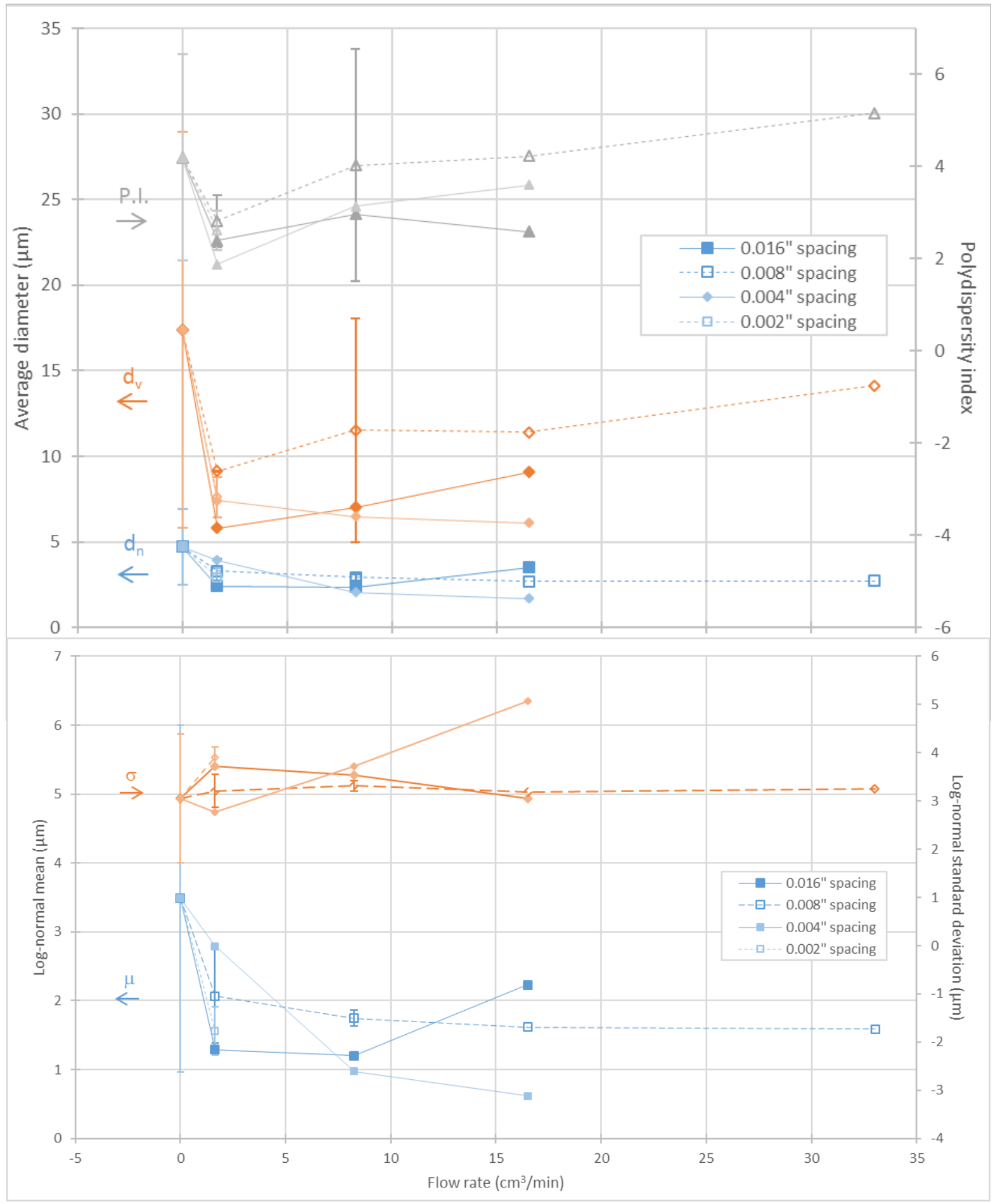

Figure 4-44: Effects of flow through hemisphere-in-cone die on morphology. Top $-d_{\mathrm{n}}, d_{\mathrm{v}}$, and P.I. for extensional flow blending of 5\% HDPE/PS through the hemisphere-in-cone die with increasing flow rate. Bottom - Mean and standard deviation from log-normal fits of the discrete histograms. Error bars represent \pm 1 standard deviation across 3 replicate cross sections, when applicable. 
The smallest useful spacer shim of 0.004 in yielded submicron size log-normal $\mu$, but as expected, required larger pressure drop than the other configurations with larger spacer thicknesses. The pressure drop data are shown in Figure 4-45. As discussed, the lowest flow rate was the only one that a sample was able to be acquired using the 0.002 in $(0.051 \mathrm{~mm})$ spacer. Also, each curve is quite linear on a log-log plot, except for the $100 \mathrm{~mm} / \mathrm{min}, 17$ $\mathrm{cm}^{3} / \mathrm{min}$, flow rate through the hemisphere-in-cone die with 0.016 in $(0.406 \mathrm{~mm})$, which was higher than expected, possibly due to clogging or flow instability effects due to melt fracture.

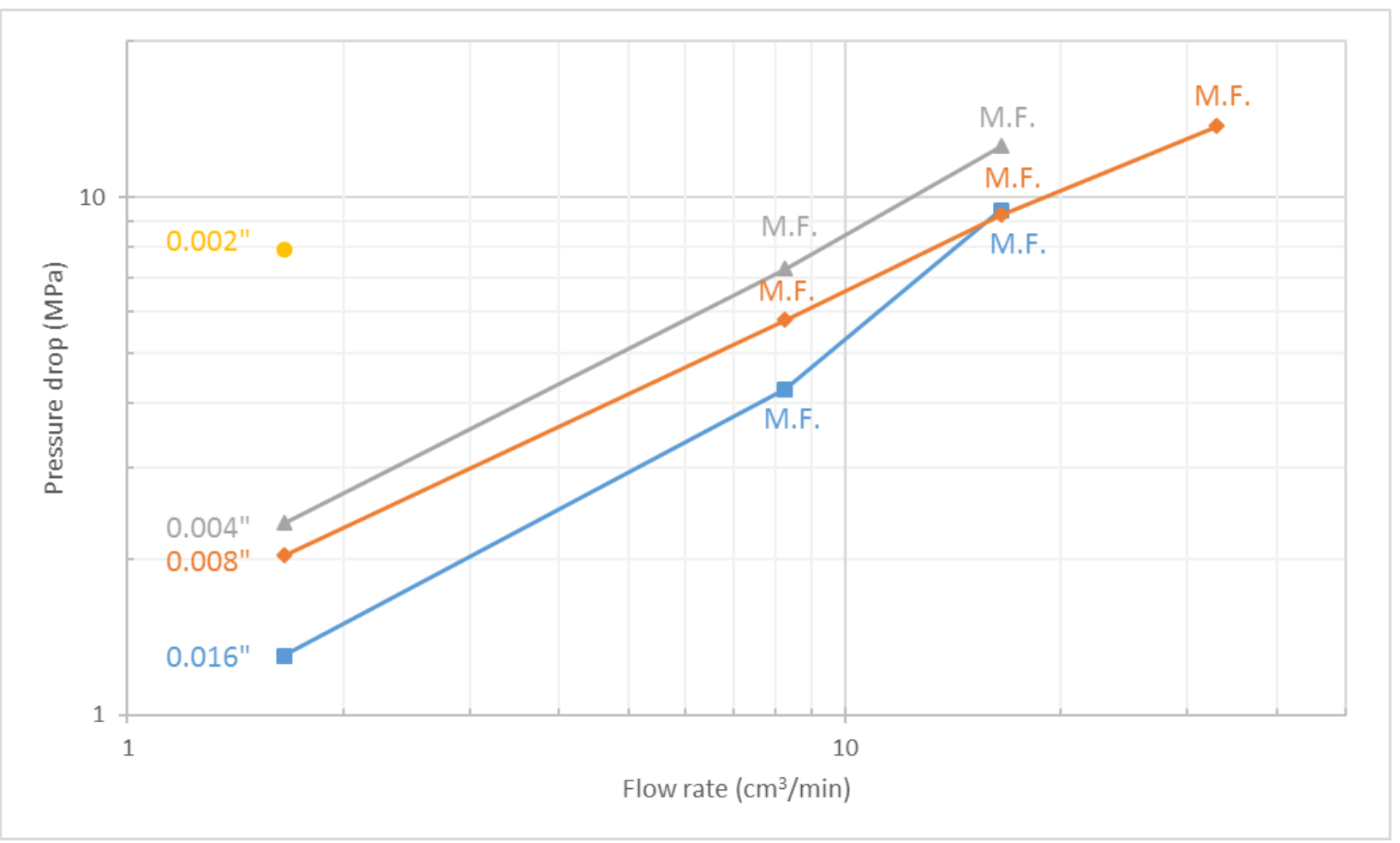

Figure 4-45: Pressure drop through hemisphere-in-cone die. Pressure drop and notation of melt fracture, "M.F." for flow through the hemisphere-in-cone die with various spacer thicknesses listed to the left of each data set. 


\subsubsection{Disk + Hemisphere Dies}

The final extensional flow cell (EFC) that was fabricated is the disk + hemisphere die, described in Section 3.3.2.5. This die combined the disk-in-tube die with the hemisphere-incone die by adding a second insert that wrapped around the hemisphere-in-cone insert. The entire assembly still nested within the converging cone die with $0.559 \mathrm{~mm}$ exit diameter, and thereby produced three stretching episodes per pass through the die. The gap between the disk and the straight inlet tube walls was again minimized within fabrication means to about 0.001 in $(0.025 \mathrm{~mm})$, but instead of straight walls on the disk, the walls tapered to enhance the extensional flow fields and detract from the shear flow contributions in this first stretching episode. The second and third stretching episodes were identical to those of the hemispherein-cone die, and again allowed for various spacers to be used to set the gap between the hemisphere and converging cone wall.

The results of flow through the disk + hemisphere die on the morphology of the $5 \%$ HDPE/PS blend are shown in Figure 4-46. The die significantly reduced $d_{v}$ for all the spacers used, without much effect of spacer thickness or flowrate, but most of the other results were seemingly errant. For example, the spacer configuration that had the most pronounced effect on $d_{\mathrm{n}}$ was the largest spacer thickness, 0.016 in $(0.406 \mathrm{~mm})$, and for the log-normal $\mu$ and $\sigma$, the 0.002 in and 0.008 in spacers yielded similar results, where the mean increased but the standard deviation decreased, while the 0.004 in and 0.016 in spacers yielded similar results to one another which were opposite to those for the other two spacer configurations. 


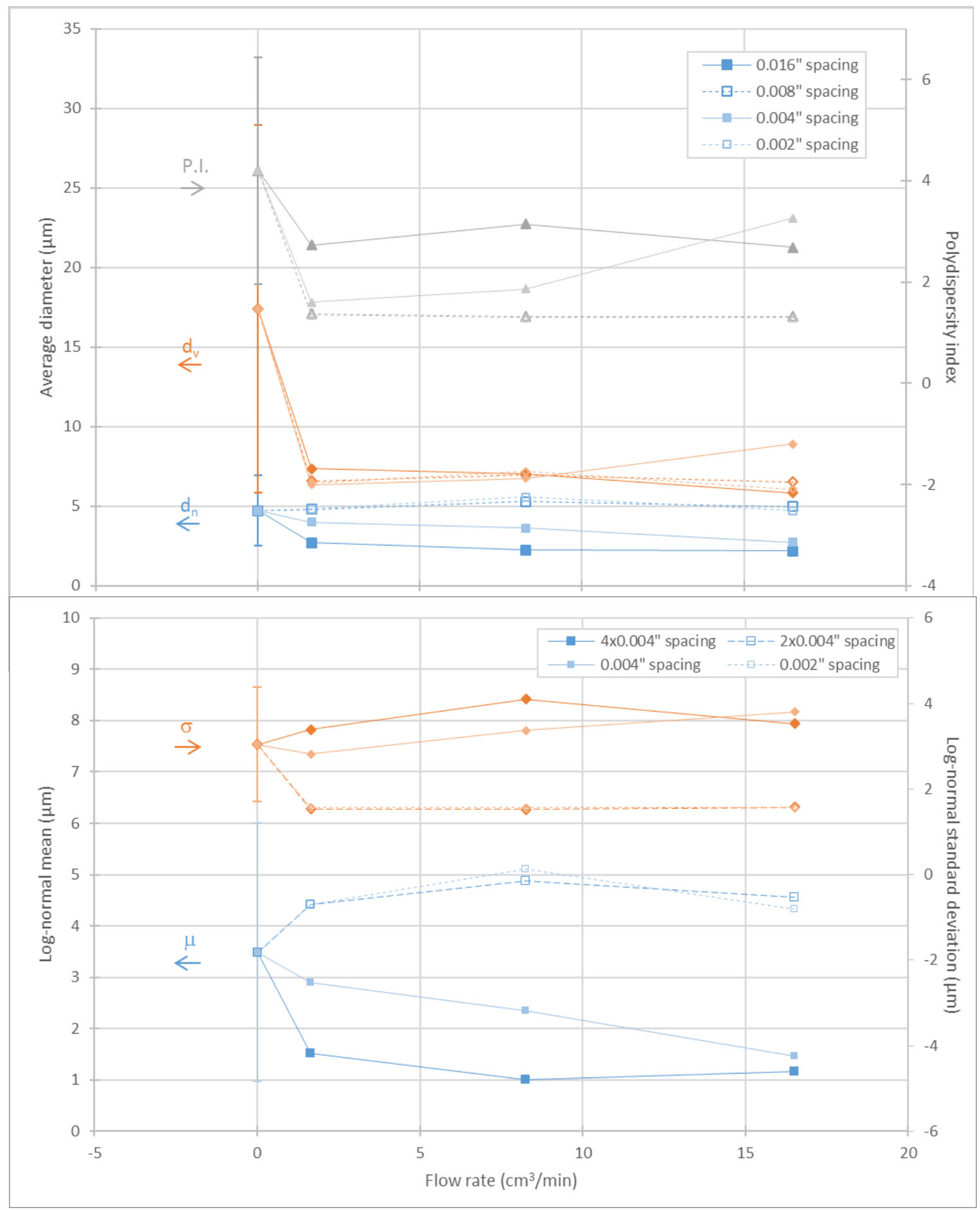

Figure 4-46: Effects of flow through disk+ hemisphere die on morphology. Top $-d_{\mathrm{n}}, d_{\mathrm{v}}$, and P.I. for extensional flow blending of 5\% HDPE/PS through the disk + hemisphere die with increasing flow rate. Bottom - Mean and standard deviation from log-normal fits of the discrete histograms. Error bars represent \pm 1 standard deviation across 3 replicate cross sections, when applicable. 
To further corroborate the assumption that the results observed were errant, one should note that the number of data points that were able to be collected was larger than that for the hemisphere-in-cone die. Figure 4-47 shows the pressure drop for each data point. The pressure drop is relatively linear with respect to flow rate on a log-log plot and it is higher than that for the hemisphere-in-cone die, but the number of flow rates that were processable and the little occurrence of melt fracture indicate that the gaps that were set in the die were not achieved in practice. Specifically, the second stretching episode, between the hemisphere insert and the converging cone wall, was expected to be the strongest contributor of extensional flow in the die, but the data indicate that the spacers were not being compressed completely and the gap present in the die was much larger than the gap setting that was attempted. The disk-in-tube secondary insert that wrapped around the hemisphere-in-cone insert was very tight, and the flow stresses alone were not adequate in ensuring that the entire assembly was pushed fully into position in the die. This is a valuable lesson learned in the design of EFCs, especially because one of the primary goals of this work was to study the potential in creating simpler, easier to use EFCs that may have better acceptance in industry. 


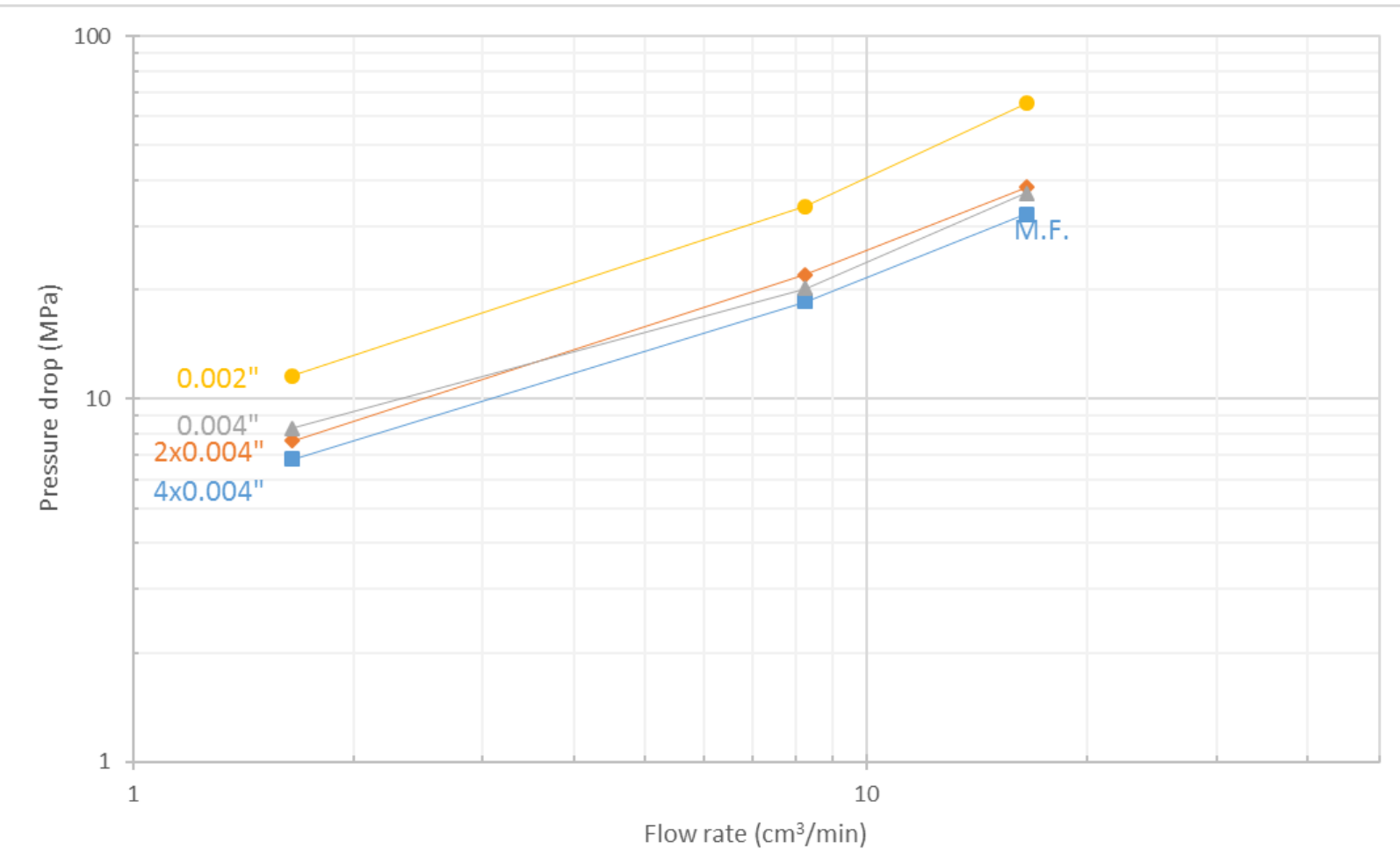

Figure 4-47: Pressure drop through disk + hemisphere die. Melt fracture "M.F." is denoted, and curves are labeled based on the spacers used to set the gap in the second stretching episode, between the hemisphere insert and the converging cone wall.

\subsubsection{Selective Dissolution of the PS Matrix}

To better elucidate the presence of elongated fibrils in the extensional flow-blended samples, the PS matrix was selectively dissolved using toluene, and the dispersed HDPE domains were filtered from the solution and imaged using SEM. Representative images of these samples are shown in Figure 4-48 and Figure 4-52 for blends processed through the orifice die with $0.559 \mathrm{~mm}$ orifice diameter and through the hemisphere-in-cone die with 0.004 in $(0.102 \mathrm{~mm})$ spacer, both at $100 \mathrm{~mm} / \mathrm{min}$ or $17 \mathrm{~cm}^{3} / \mathrm{min}$. Both of these dies significantly affected the morphology, and as can be seen, elongated fibrils are present in both. The blend from the orifice die contained more fibrils with very small diameters, while the blend from the hemisphere-in-cone die contained larger elongated fibrils. These were frozen in place in the extrudate strand due to quenching in water. 


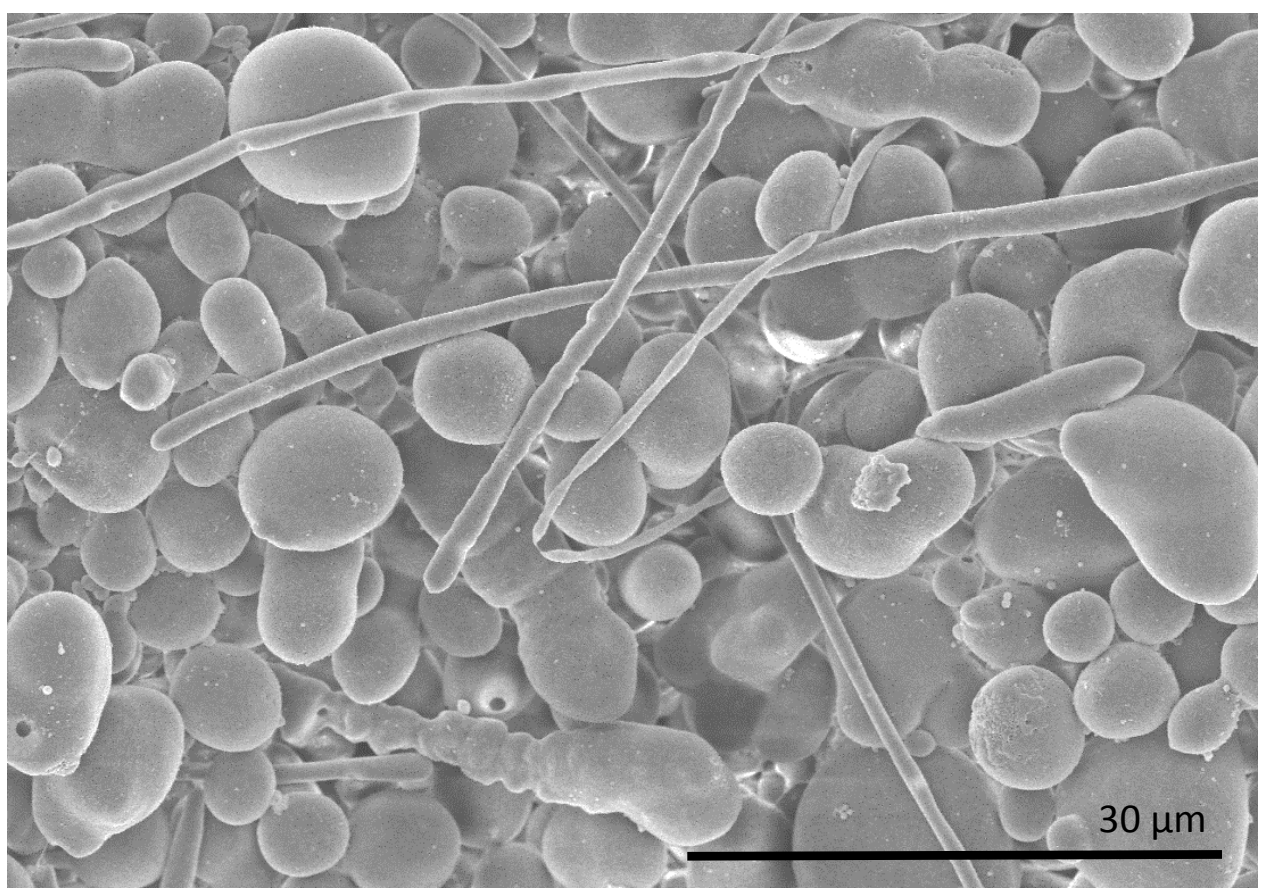

Figure 4-48: HDPE drops and thin threads after orifice, $0.559 \mathrm{~mm}, 17 \mathrm{~cm} / \mathrm{min}$.

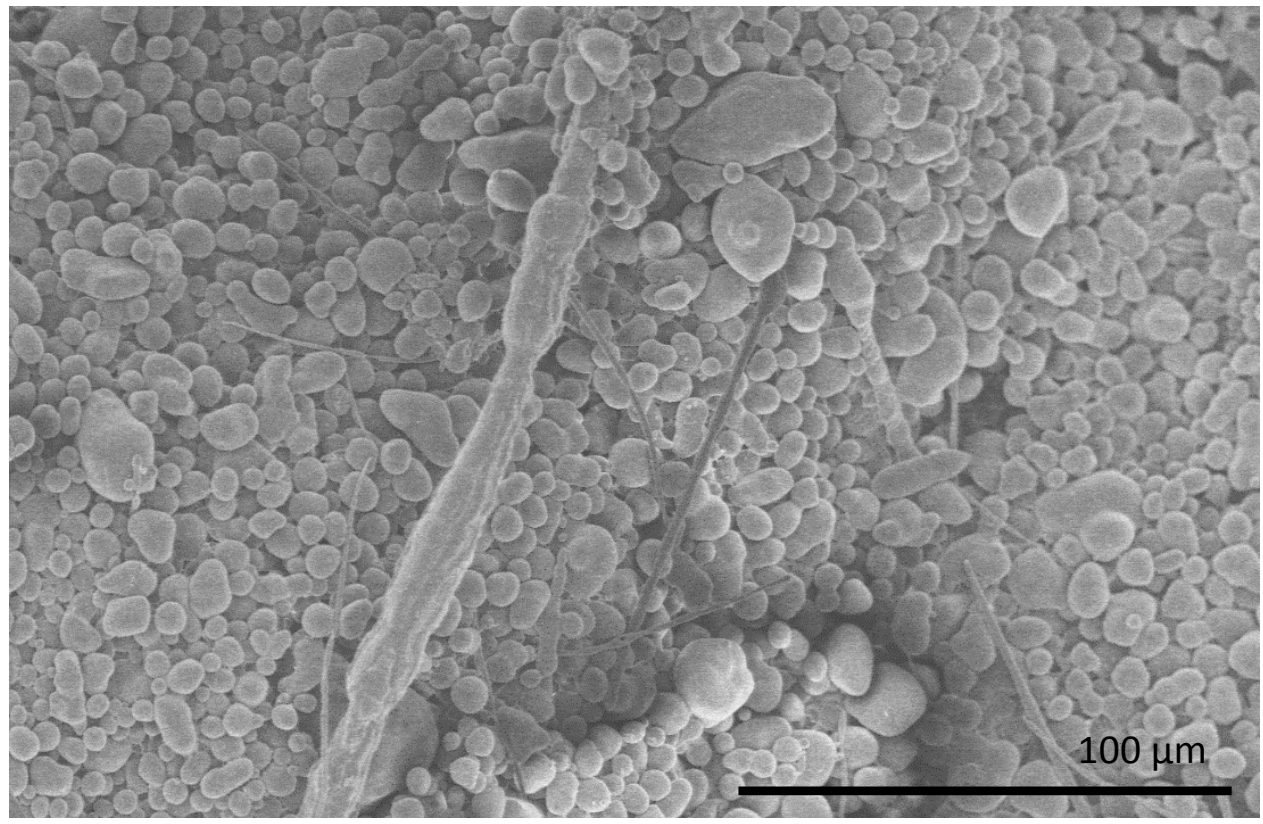

Figure 4-49: HDPE drops and threads after hemisphere-in-cone, 0.004 " spacer, $17 \mathrm{~cm}^{3} / \mathrm{min}^{\text {. }}$

Furthermore, alternative breakup mechanisms were observed as well with this method of sample preparation. The dominant breakup mechanism is capillary breakup of elongated dispersed threads or fibrils. The very fine diameter in the blend from the orifice die may 
indicate that the blend included bead-string breakup, in which surface disturbances do not grow sinusoidally, but rather create beads separated by very thin string-like strand sections. This is observed in highly elastic materials and generally retards breakup compared to the more typical sinusoidal, capillary-instability thread breakup. In addition, tip streaming was observed in that same sample, shown in Figure 4-50, and this is another indication that fluid elasticity is important in this blend system.

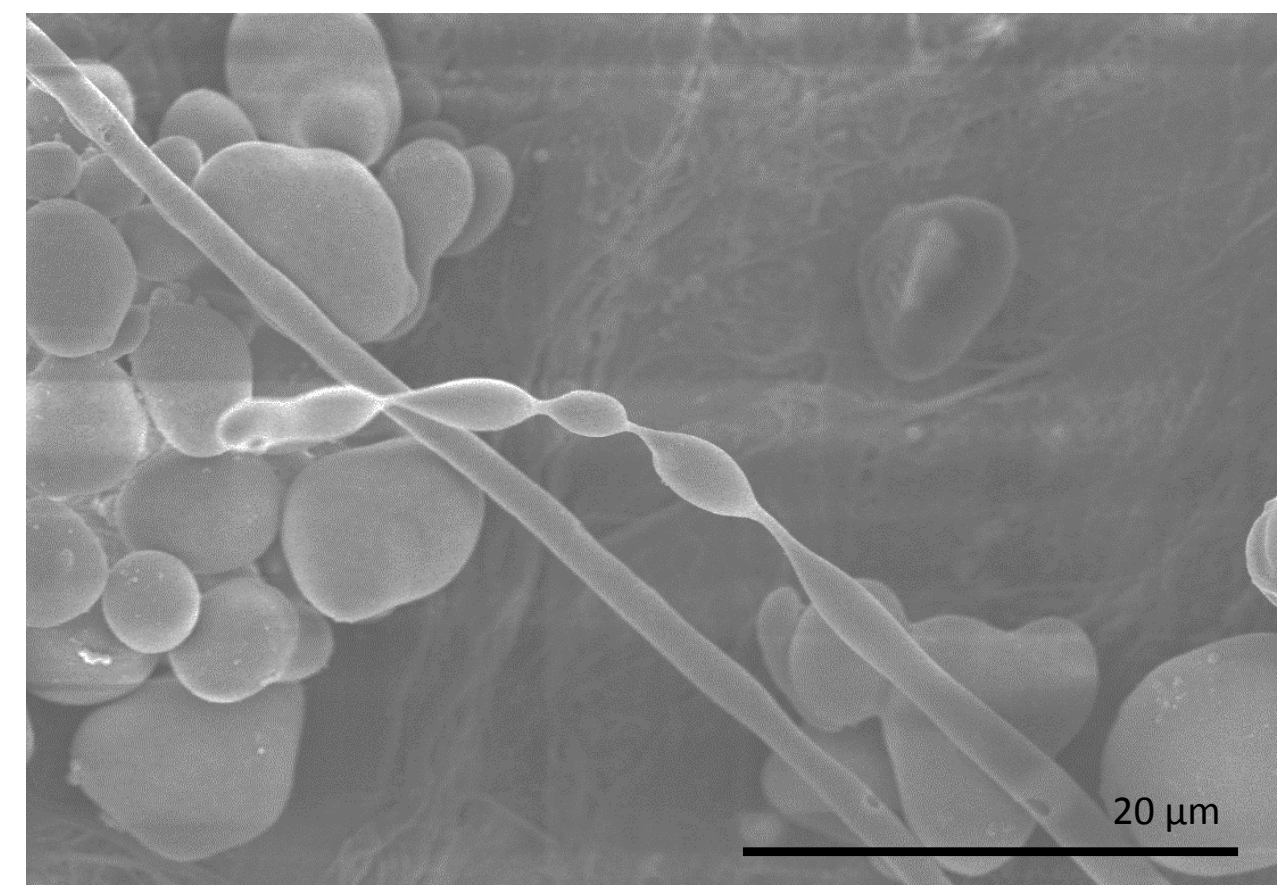

Figure 4-50: Tip streaming on an HDPE thread after orifice, $0.559 \mathrm{~mm}, 17 \mathrm{~cm}^{3} / \mathrm{min}$.

\subsubsection{Overall Comparisons}

Extensional flow blending was studied predominantly by designing, fabricating, and testing simple converging-geometry nozzles which impart the blends to axisymmetric extensional flow. The entry profile was varied from a cone angle of $180^{\circ}$, representing an orifice, to a converging cone with straight walls and a half-cone angle of about $45^{\circ}$. The exit diameter of these converging-geometry dies was varied, which varied the total extensional strain through the dies, and the flow rate was also varied, which directly varied the extensional 
strain rate. Increasing total strain through the dies produced finer morphologies, but the effect was at the expense of pressure drop through the die. Increasing flow rate also produced incrementally finer morphologies, with an optimum flow rate given by the point at which the extensional strain rate was high, yet the residence time in the converging section was not too short, especially in comparison to the elastic relaxation time of the dispersed polymer phase.

Additionally, inserts of various geometries were designed, fabricated, and tested which nest within a converging cone nozzle and impart the blends to an additional stretching episode. This stretching episode was comprised of a significant portion of planar extensional flow, which is more efficient for dispersion than axisymmetric extensional flow, and the strength of the extensional flow fields produced was able to be controlled by the gap between the insert and the converging cone wall. Three different geometries using inserts in a converging cone die were investigated: 1 ) disk-in-tube, consisting of a straight-walled disk that nested within the straight-walled tube section of the nozzle, upstream of the converging cone section; 2) hemisphere-in-cone, consisting of a hemispherical insert that nested within the converging section of a converging cone die, the gap between them set by adding or removing spacers upon which the insert sat; and 3) disk + hemisphere inserts, where a second insert was added to the hemisphere-in-cone insert that wrapped around it and created an additional upstream stretching episode between the tapered walls of the disk and the straight walls of the tube section of the die. The most advantageous die design was the hemisphere-in-cone die. The disk-in-tube die produced unsatisfactory pressure drops which did not produce significantly finer morphologies, and the disk + hemisphere die produced errant results which indicated that keeping the bulky insert concentric to the converging cone die axis and ensuring that the insert was forced completely to the bottom of the die, to set a known gap between the hemisphere insert and the converging cone wall, were difficult to achieve in practice. Furthermore, the hemisphere-in-cone die has the favorable option of altering the gap for the most intense stretching episode present between the hemisphere and the converging cone wall.

The results of extensional flow blending using all of the dies investigated in this work using a piston speed of $100 \mathrm{~mm} / \mathrm{min}$, flow rate $17 \mathrm{~cm}^{3} / \mathrm{min}$, on morphology are compiled in Figure 4-51, and the pressure drop and notation of melt fracture are given in Figure 4-52. The 
simplest geometries actually produced the most pronounced effects on the morphology, and required the smallest pressure drop. This was one of the main goals of this work, to characterize the potential in significantly affecting morphology development in polymer processing using much simpler geometries that are easier and more energy-efficient to use. The simple orifice die with $0.559 \mathrm{~mm}$ orifice diameter produced the finest $d_{\mathrm{v}}$ at the flow rate shown and required the smallest pressure drop. The hemisphere-in-cone die using the 0.004 in (0.102 mm) spacer, however, produced a very similar morphology and did not require a significant increase in pressure drop. With optimization of dies of that type, including eliminating potentially stagnant regions in the die, the hemisphere-in-cone die appears to have the best potential for future impact. 


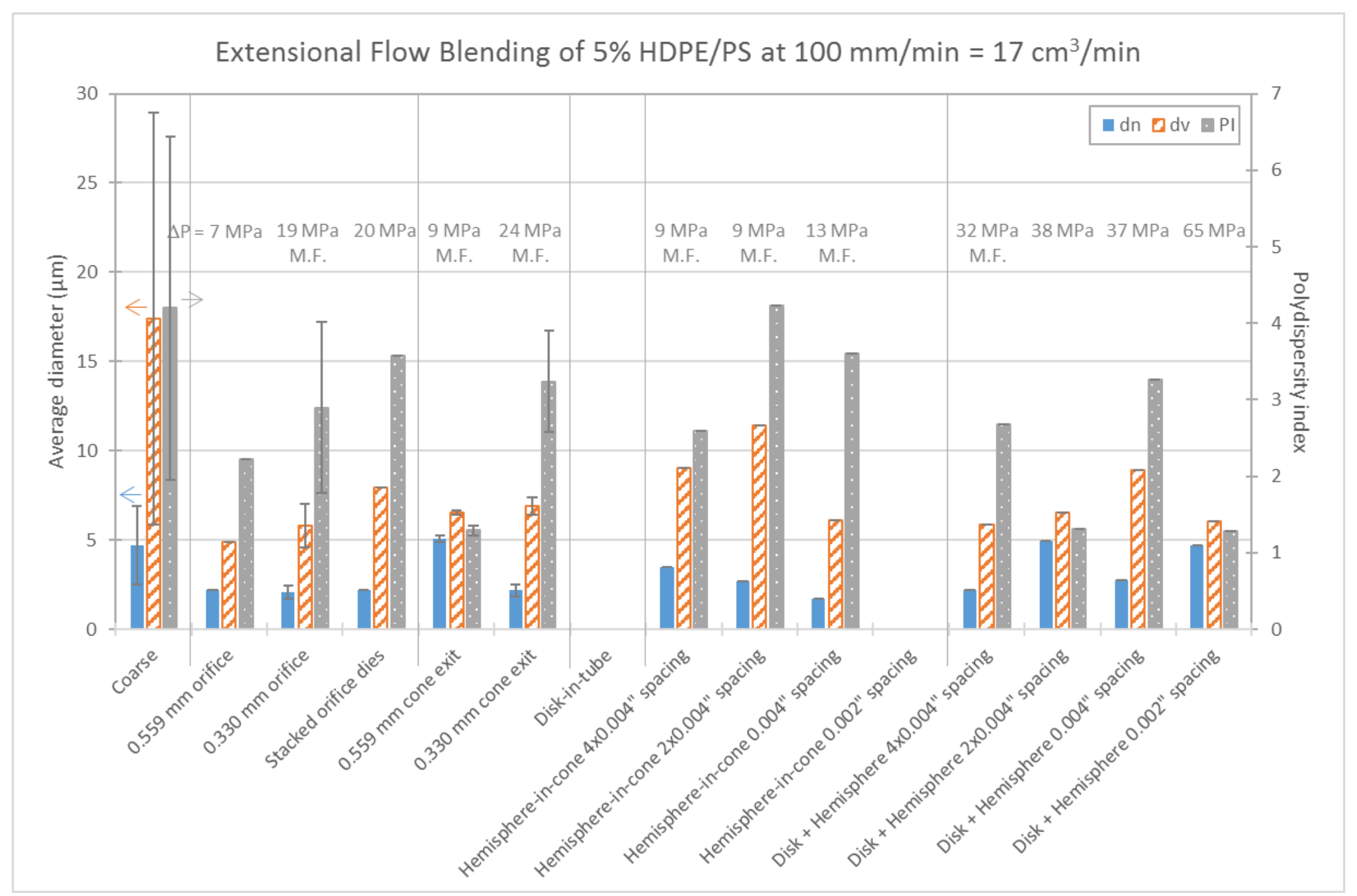

Figure 4-51: Comparison of morphology after blending through all of the EFCs 


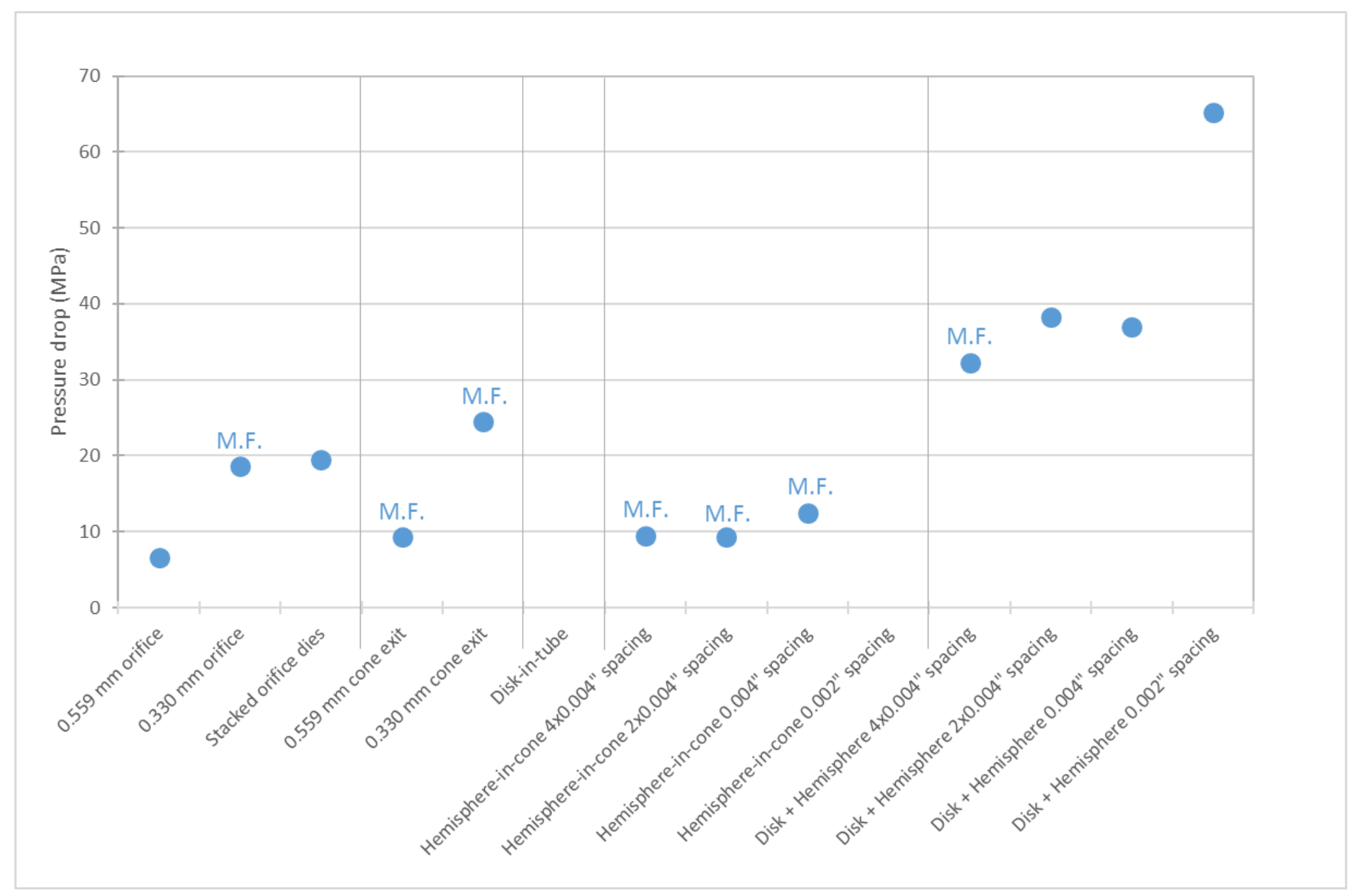

Figure 4-52: Comparison of pressure drop after blending through all of the EFCs 


\subsection{Nanoparticle Stabilization}

In this work, a blend system was selected that was incompatible both physically and thermodynamically. Physical incompatibility was defined by the high viscosity ratio for all shear rates of processing, and it was overcome at least in part using physical mixing techniques, namely the use of extensional flow mixing cells to impart strong dispersive extensional flow fields onto the blends. The blend systems of interest in this work are also thermodynamically incompatible, as defined by their immiscibility with one another and the significant interfacial tension between the phases. Thermodynamic incompatibility is accounted for in the polymer

engineering and science field by compatibilization, usually with surfactants, block copolymers, or reactive agents, all of which tend to migrate to the interface between the polymers and modify the overall surface kinetics and energy of the blend. A relatively novel approach was taken to accomplish this in this work, wherein solid nanoparticles with tuned surface chemistries are used to modify the interfacial properties of the blend and serve as compatibilizers. The term nanoparticle stabilization refers to the stabilization of the morphology produced by the processing of Section 4.2 against the coarsening effects of coalescence. Several nanoparticle grades were used in this work and the efficacy and challenges of the nanoparticle stabilization route are described in this section.

\subsubsection{Properties of Fumed Nanosilica}

The nanoparticles chosen for the investigations of this work were fumed nanosilica, offered by and graciously donated by Evonik Industries. Fumed nanosilica is a form of silica that is collected from the top of the sintering process. It is typically received in the form of a fine, low-bulk density powder. The primary particles are spherical and nonporous with diameters on the order of 7-20 $\mu \mathrm{m}$. However, the as-received material is inevitably agglomerated, and its dispersion down to aggregates and separate primary particles is a challenge that has been the focus of much academic and industrial research efforts for some time. To exploit the attractive properties of "nano," the nanomaterial must first be truly of the nano-scale. The criticality of 
dispersing the nano-phase is an often overlooked, but crucial part of working with nanomaterials.

Scanning electron microscopy (SEM) images of the as-received Aerosil ${ }^{\circledR}$ R104 from Evonik Industries, which has a hydrophobic surface treatment, are shown in Figure 4-53. The agglomerates are on the order of several microns in size, and they are comprised of aggregates that are typically about 50-300 $\mathrm{nm}$ in size. The primary particle size of the nanosilica, along with most of the other grades investigated in this work, is about $12 \mathrm{~nm}$. The level of dispersion of the nanomaterial is discussed further throughout this section.
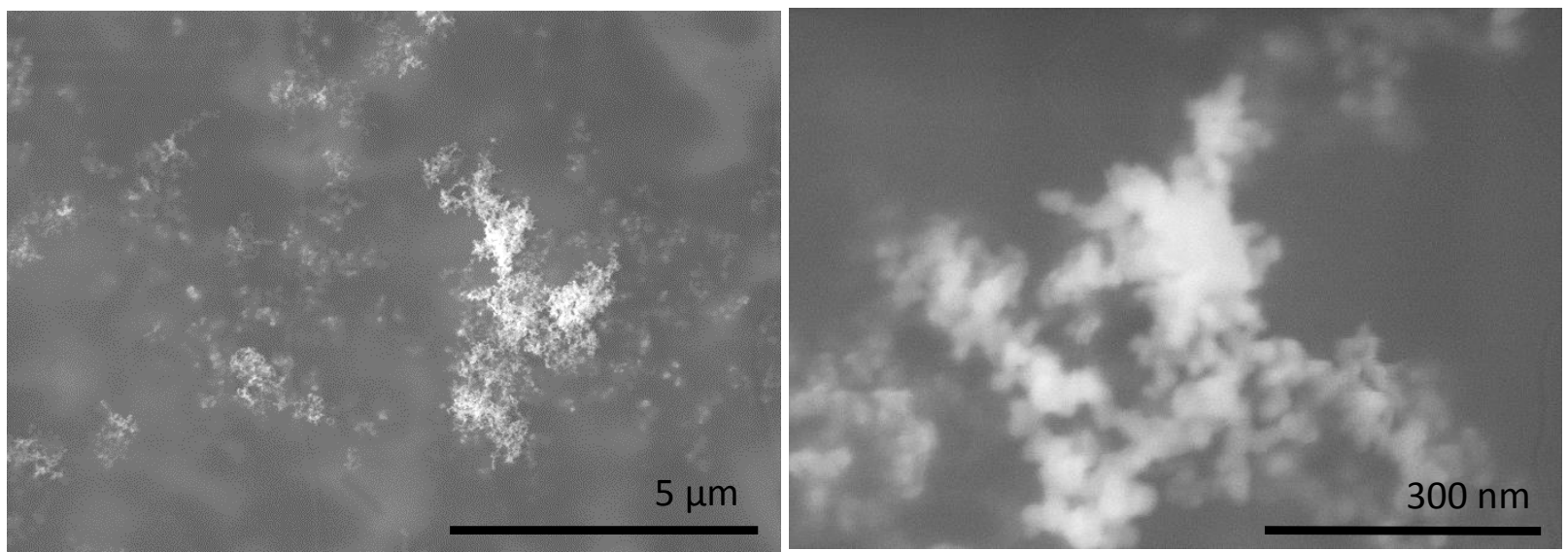

Figure 4-53: As-received Aerosil ${ }^{\circledR}$ R104 in agglomerated form

Several different grades of surface-treated nanosilica grades were obtained from Evonik Industries; the full range of surface polarity was covered in an attempt to identify a surface treatment that would cause the nanoparticles to thermodynamically bind to the polymerpolymer interface, where they would have the most influence over the behavior of the blend, including the robustness of the morphology to the coarsening of coalescence. The properties of the nanosilica grades used in this work are given in Table 4-17. Fumed nanosilica without surface treatment has a hydrophilic surface, imparted onto it by the presence of oxygen-rich moieties, such as silanol and siloxane. The Aerosil 200 grade was untreated, with a hydrophilic surface and a primary particle size of $12 \mathrm{~nm}$. Eight grades of Aerosil were also obtained, all of which were surface treated by Evonik Industries to impart a more hydrophobic surface onto the 
nanomaterial. This makes the grades much more compatible with polymer matrices because polymers are typically relatively nonpolar with hydrophobic surfaces. Most of the grades maintained a primary particle size of $12 \mathrm{~nm}$, but grades of $7 \mathrm{~nm}$ and $16 \mathrm{~nm}$ were also investigated. The surface treatments involved the addition of nonpolar silanes and siloxanes to the surface of the nanomaterial. The surface-treating molecules ranged from small molecules to those containing 8- and 16-member chains, to a polydimethylsiloxane (PDMS) coating. The order that the grades are listed in Table 4-17 is the speculated order from most hydrophilic to most hydrophobic. The surface tension and surface polarity properties were obtained from various literature references, where the surface-treating molecule was generally assumed to cover the entire surface of the nanoparticle homogeneously. The validity of this assumption was not tested, though it is assumed that parts of the surface are rich and lean in the surfacetreating molecule, which is typically simply physically or chemically adsorbed to the surface. 
Table 4-17: Properties of nanosilica grades

\begin{tabular}{|c|c|c|c|c|c|c|c|c|c|}
\hline $\begin{array}{l}\text { Aerosil } \\
\text { grade }\end{array}$ & $\begin{array}{c}\text { BET } \\
\text { specific } \\
\text { As } \\
\left(\mathrm{m}^{2} / \mathrm{g}\right)\end{array}$ & $\begin{array}{l}\text { Particle } \\
\text { size } \\
\text { (nm) }\end{array}$ & $\begin{array}{c}\gamma_{\mathrm{s}} \\
(\mathrm{mN} / \mathrm{m})\end{array}$ & $\begin{array}{c}\gamma^{\mathrm{d}} \\
(\mathrm{mN} / \mathrm{m})\end{array}$ & $\begin{array}{c}\gamma^{p} \\
(\mathrm{mN} / \mathrm{m})\end{array}$ & $\begin{array}{l}\text { Surface } \\
\text { polarity } \\
(\%)\end{array}$ & $\begin{array}{c}\mathrm{d} \gamma / \mathrm{dT} \\
{[\mathrm{mN} /(\mathrm{m}} \\
\mathrm{K})]\end{array}$ & Surface treatment & References \\
\hline 200 & $200 \pm 25$ & 12 & 80 & 29.4 & 50.6 & 63 & -0.1 & $\begin{array}{l}\text { None, silanol and siloxane } \\
\text { moieties render surface } \\
\text { hydrophilic } \\
\end{array}$ & $\begin{array}{l}\text { [24] for size and surface } \\
\text { tension. [73] for size and } \\
\text { surface moieties. }\end{array}$ \\
\hline R974 & $170 \pm 20$ & 12 & $39-44$ & & & & -0.1 & $\begin{array}{l}\text { Dimethyldichlorosilane } \\
\text { (DDS or DMDCS) } \\
\mathrm{Cl}-\left.\right|_{\mathrm{Cl}} ^{\mid}\end{array}$ & $\begin{array}{l}\text { [74] for surface tension and } \\
\text { used } d \gamma / d T \text { value for } C_{16} \\
\text { coating. [29] for size. }\end{array}$ \\
\hline R972 & $110 \pm 20$ & 16 & $39-44$ & & & & -0.1 & $\begin{array}{l}\text { Dimethyldichlorosilane } \\
\text { (DDS or DMDCS) } \\
\mathrm{Cl}-\mathrm{Si}- \\
\mathrm{Cl}\end{array}$ & $\begin{array}{l}\text { [74] for surface tension and } \\
\text { used } d \gamma / d T \text { value for } C_{16} \\
\text { coating. [75] [29] [28] for size. }\end{array}$ \\
\hline R816 & $190 \pm 20$ & 12 & 33 & 31 & 2 & 6.3 & -0.1 & Hexadecylsilane $\left(\mathrm{C}_{16}\right)$ & $\begin{array}{l}\text { [76] for surface tension. [77] } \\
\text { for size. }\end{array}$ \\
\hline R106 & $250 \pm 30$ & 7 & 18.4 & 17.7 & 0.7 & 4.0 & -0.081 & $\begin{array}{l}\text { Octamethylcyclotetra- } \\
\text { siloxane (OMCTS or D4) } \\
\end{array}$ & $\begin{array}{l}\text { [78] for surface tension, but } \\
\text { used surface polarity from } \\
\text { value for PDMS. [29] for size. }\end{array}$ \\
\hline
\end{tabular}


Table 4-17 (Continued): Properties of nanosilica grades

\begin{tabular}{|c|c|c|c|c|c|c|c|c|c|}
\hline $\begin{array}{l}\text { Aerosil } \\
\text { grade }\end{array}$ & $\begin{array}{c}\text { BET } \\
\text { specific } \\
\text { As }^{2} \\
\left(\mathrm{~m}^{2} / \mathrm{g}\right)\end{array}$ & $\begin{array}{l}\text { Particle } \\
\text { size } \\
\text { (nm) }\end{array}$ & $\begin{array}{c}\gamma_{\mathrm{s}} \\
(\mathrm{mN} / \mathrm{m})\end{array}$ & $\begin{array}{c}\gamma^{d} \\
(\mathrm{mN} / \mathrm{m})\end{array}$ & $\begin{array}{c}\gamma^{p} \\
(\mathrm{mN} / \mathrm{m})\end{array}$ & $\begin{array}{l}\text { Surface } \\
\text { polarity } \\
(\%)\end{array}$ & $\begin{array}{c}\mathrm{d} \gamma / \mathrm{dT} \\
{[\mathrm{mN} /(\mathrm{m}} \\
\mathrm{K})]\end{array}$ & Surface treatment & References \\
\hline R104 & $150 \pm 25$ & 12 & 18.4 & 17.7 & 0.7 & 4.0 & -0.081 & $\begin{array}{l}\text { Octamethylcyclotetra- } \\
\text { siloxane (OMCTS or D4) } \\
\qquad /\end{array}$ & $\begin{array}{l}\text { [78] for surface tension, but } \\
\text { used surface polarity from } \\
\text { value for PDMS. [29] for size. }\end{array}$ \\
\hline R208 & $115 \pm 25$ & $12-14$ & 19.8 & 19.0 & 0.8 & 4.0 & -0.048 & 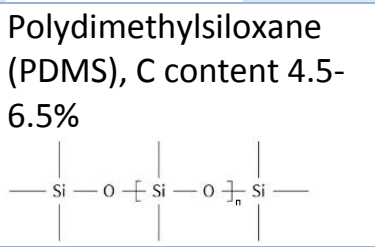 & $\begin{array}{l}\text { [79] for surface tension. Size } \\
\text { estimated from BET } A_{s} \text { as } \\
\text { compared to those of Aerosil } \\
\text { R972 and Aerosil R104. }\end{array}$ \\
\hline R812S & $220 \pm 25$ & 7 & 18.16 & & & & -0.085 & $\begin{array}{l}\text { Hexamethyldisilazane } \\
\text { (HMDS or HMDZ) } \\
\qquad \mathrm{H} \\
-\mathrm{Si}-\mathrm{N}-\mathrm{S}-\end{array}$ & $\begin{array}{l}\text { [78] for surface tension. [28] } \\
\text { for size. }\end{array}$ \\
\hline R8200 & $160 \pm 25$ & 12 & 18.16 & & & & -0.085 & $\begin{array}{l}\text { Hexamethyldisilazane } \\
\text { (HMDS or HMDZ) } \\
\mathrm{Si}_{\mathrm{S}-\mathrm{N}-\mathrm{Si}}-\end{array}$ & $\begin{array}{l}\text { [78] for surface tension. [80] } \\
\text { for size. }\end{array}$ \\
\hline
\end{tabular}


To have full effect even if the thermodynamics selectively localize the nanoparticles at the polymer-polymer interface, the loading of nanoparticles must be high enough to fully cover the interface. The loading of nanosilica required to fully cover HDPE dispersed-phase drops which are assumed to be monodisperse in size for the calculation is presented in Figure 4-54, where separate curves are given based on the level of dispersion of the nanomaterial and the amount of interface coverage that is deemed necessary. The calculation simply compared the projected area of the nanoparticle phase to the interfacial area of the HDPE drops. One of the goals of this work was to achieve dispersed-phase morphologies with dispersed-phase sizes less than $1 \mu \mathrm{m}$. For this, only $0.3-0.6 \mathrm{wt} \%$ of fully dispersed nanosilica is needed, whereas at least 3 $w t \%$ and up to about 5 wt\% may be required using an aggregated nanofiller.

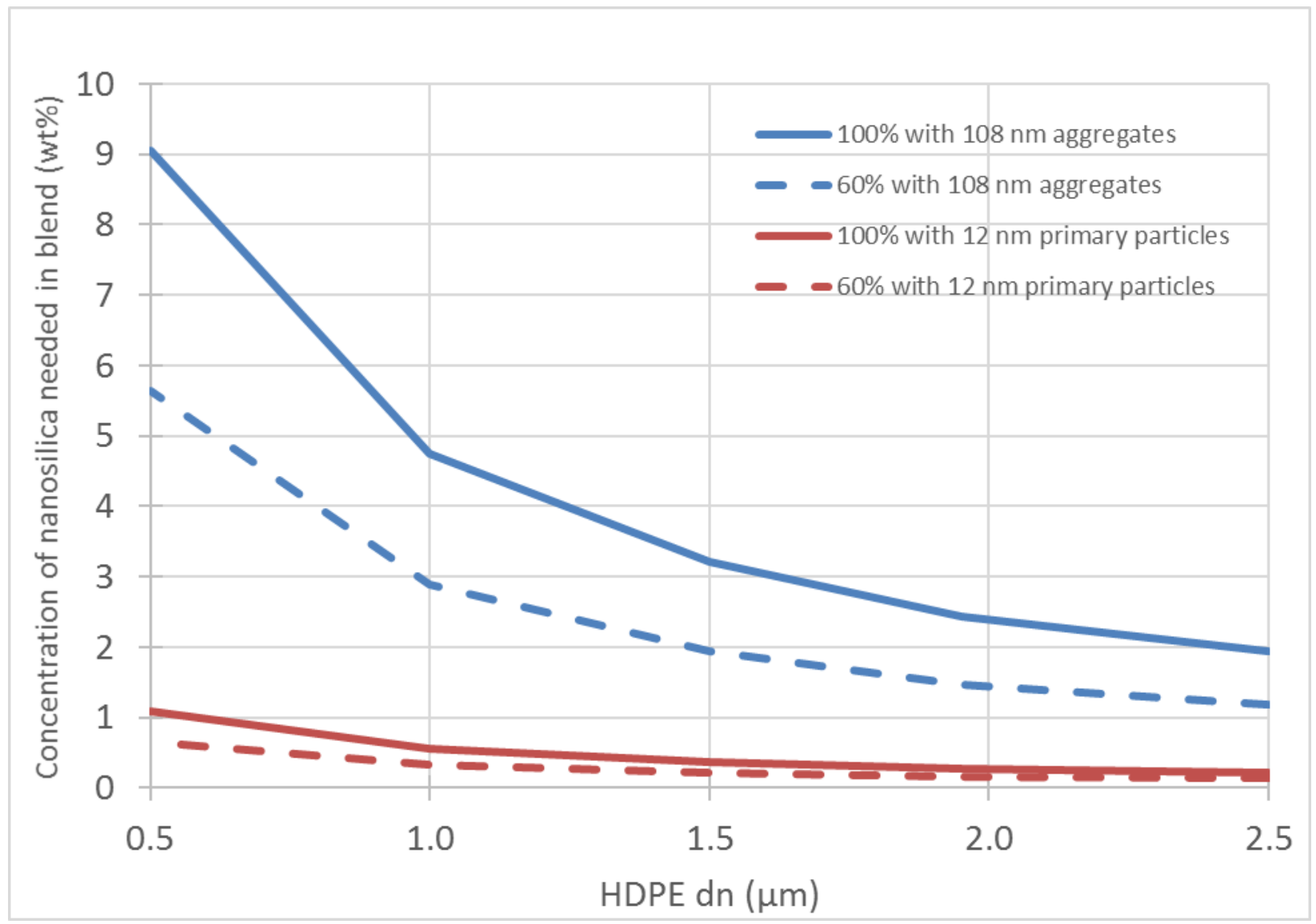

Figure 4-54: Predicted loading of nanosilica required to cover the HDPE/PS interface 


\subsubsection{Nano-Filled PC/SAN}

As in Section 4.1, a great deal of work was accomplished with the preliminary blend systems investigated in this project, and some of the results of nano-filled PC/SAN and nanofilled PS/LLDPE will be presented in this section.

\subsubsection{Thermodynamics of Nano-Filled PC/SAN}

The wettability parameter is useful in predicting the thermodynamically preferred localization of a third phase in a binary blend. It was described in Section 2.3.1 and is defined in Equation (2-54). The harmonic mean equation is useful in calculating the interfacial tension between phases, based on each of their total surface tension and its disperse and polar parts; it is given in Equation (4-3). The wettability parameter for single, isolated, non-porous, generalized nanoparticles in PC/SAN is given in Figure 4-55 based on the nanoparticle's surface tension and relative surface polarity. The window of surface properties for which nanoparticles are thermodynamically predicted to localize at the PC/SAN interface includes nanoparticles with low total surface tension but high surface polarity and those with high total surface tension but low surface polarity. These two combinations are not typically encountered because total surface tension is often directly proportional to the relative surface polarity. Nanoparticles with intermediate surface tension and surface polarity are predicted to thermodynamically bind to the PC/SAN interface. 


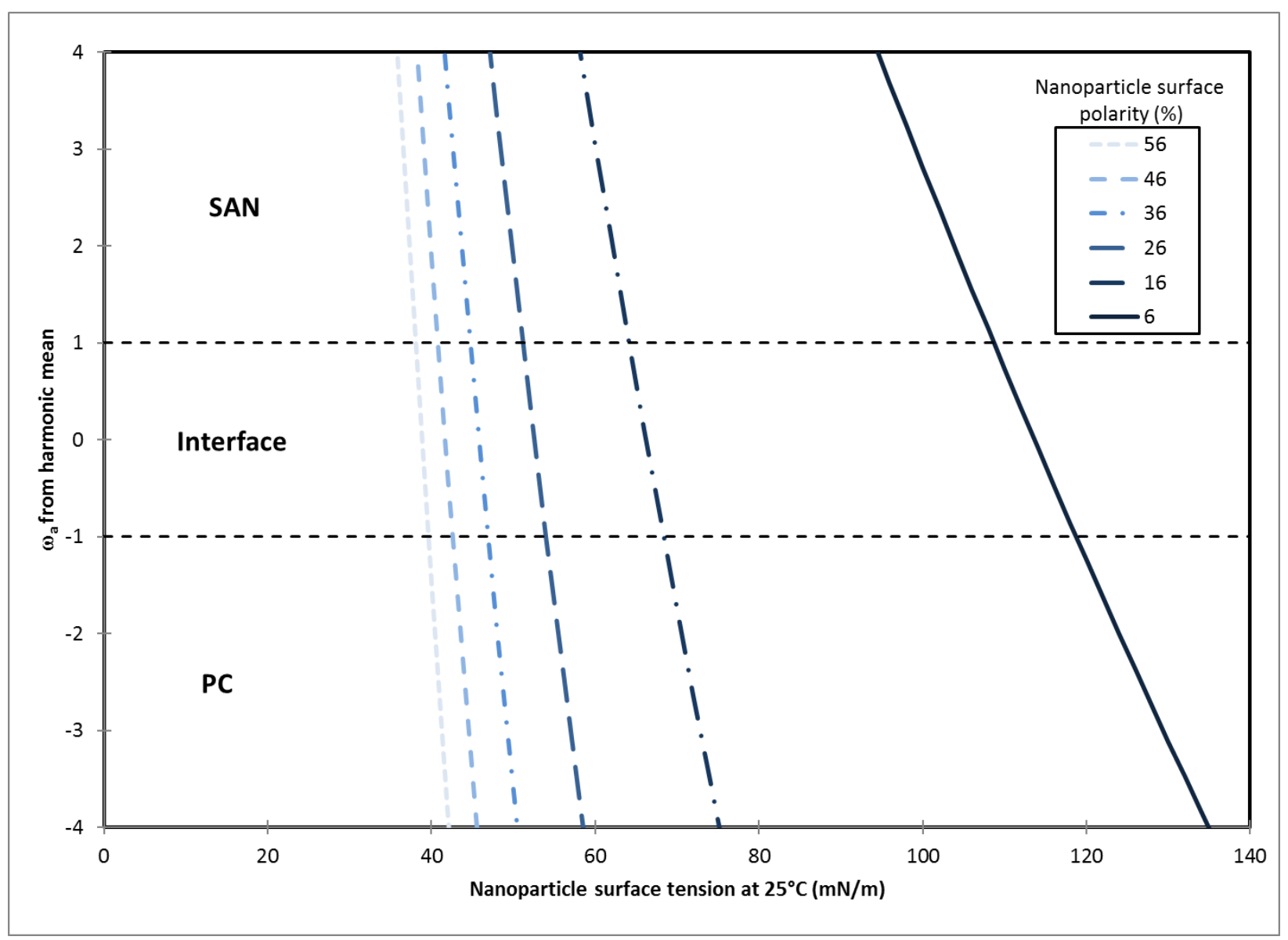

Figure 4-55: Predicted wettability of nanoparticles in PC/SAN. Thermodynamically predicted phase localization of nanoparticles of various surface tensions and surface polarities in PC/SAN at $260^{\circ} \mathrm{C}$.

\subsubsection{Nano-Filled PC/SAN Coarse Blends}

Transmission electron microscopy (TEM) was performed on PC/SAN coarse blends, prepared using one-step mixing in the internal mixer and using both the hydrophilic nanosilica, Aerosil 200, and one of the hydrophobic nanosilica grades, Aerosil R104. This is shown in Figure 4-56. Unfortunately, sufficient contrast between the two polymer phases, PC and SAN, was not achieved to allow one to distinguish the dispersed PC drops from the SAN matrix. However, the level of mixing of the nanoparticles, which are the dark phase in the images, may be investigated, including both their dispersion and distribution. Though both grades of nanosilica remain in aggregated form after processing with the internal mixer, no large agglomerates are 
observed, and the nanofiller is fairly evenly distributed throughout the polymer system. This level of mixing is better than expected using just the predominantly shear flow fields in the internal mixer.

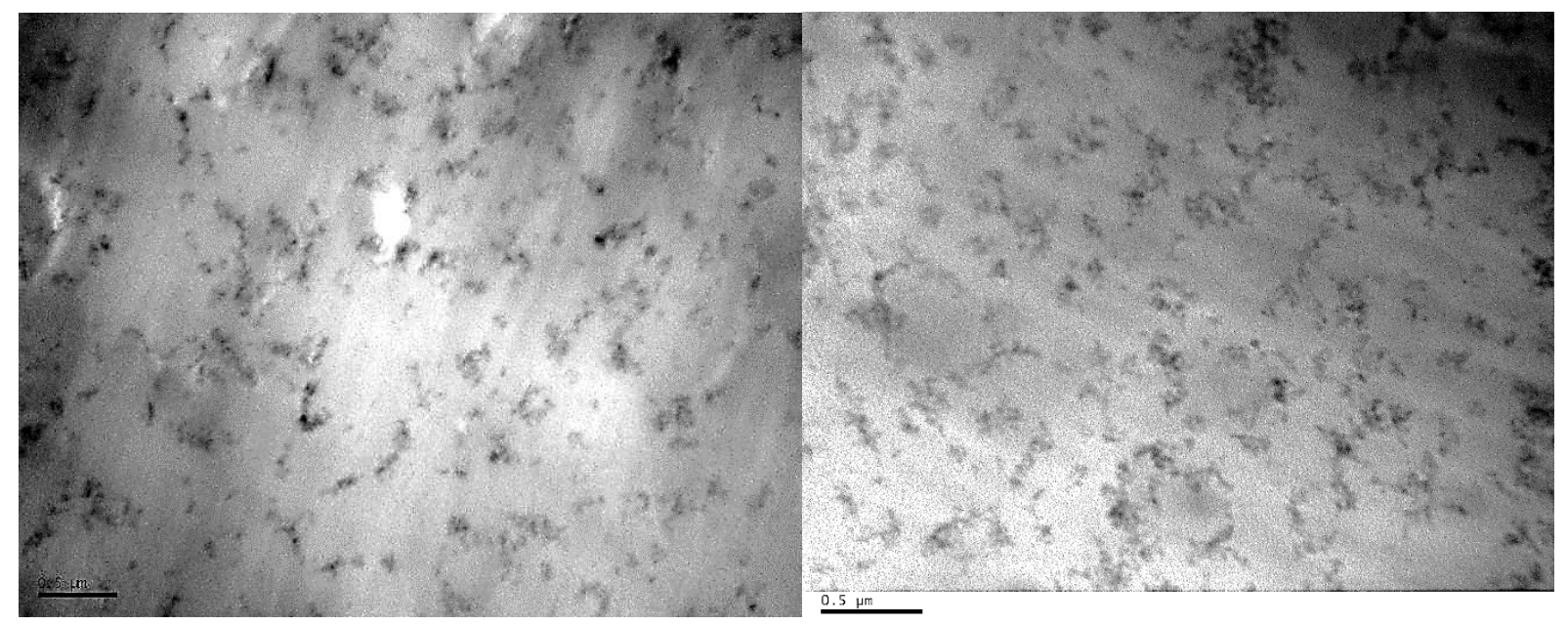

Figure 4-56: TEM of $2 \%$ nanosilica in $15 \%$ PC/SAN coarse blends. Coarse blends prepared by one-step mixing in the internal mixer: Left - Hydrophobic nanosilica, Aerosil R104; Righthydrophilic nanosilica, Aerosil 200. Scale bar in lower-left corner of each image is $0.5 \mu \mathrm{m}$.

The effects of adding nanosilica into the PC/SAN coarse blends are shown in Figure 4-57. With the hydrophobic Aerosil R104, the loading of nanosilica appears to be very important. The morphology was actually coarser for the $2 \%$ Aerosil R104 in 15\% PC/SAN blend than the unfilled $15 \%$ PC/SAN blend, whereas the morphology was finer with a loading of $7.6 \%$. For this blend system, the hydrophilic nanosilica Aerosil 200 appears to have a strong effect on the dispersed polymer phase morphology as both $2 \%$ and $4 \%$ loading of Aerosil 200 significantly decreased $d v$ compared to the unfilled blend. 


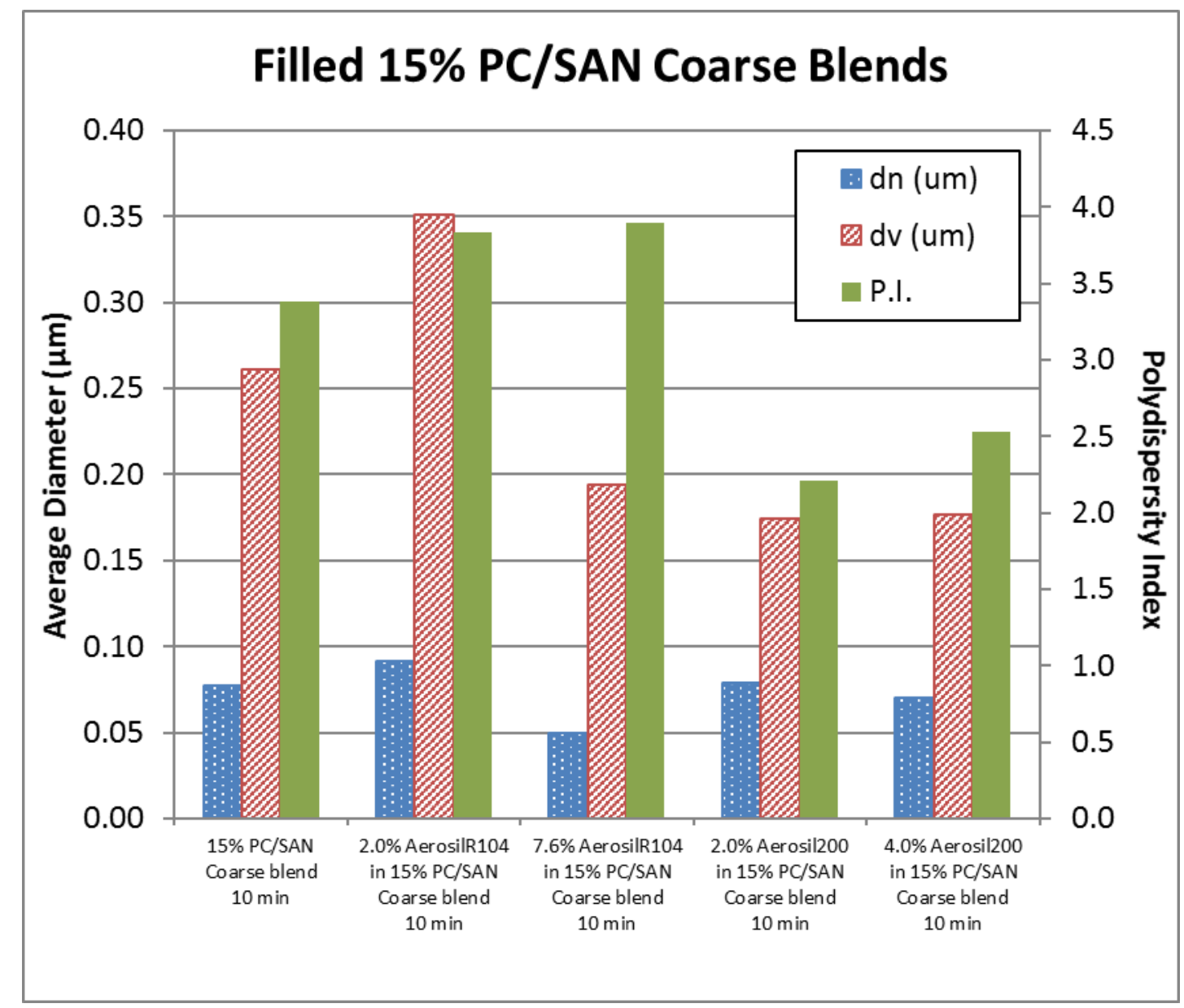

Figure 4-57: Morphology of nanosilica-filled 15\% PC/SAN coarse blends.

\subsubsection{Extensional Flow with Nanoparticle Stabilization in PC/SAN}

The effects of extensional flow blending using the stacked orifice dies on the morphology of nano-filled and unfilled 15\% PC/SAN are shown in Figure 4-58. For the unfilled blend and the $2 \%$ Aerosil R104-filled blend, extensional flow blending significantly decreased $d_{v}$, and even decreased $d_{\mathrm{n}}$ for the latter blend. However, extensional flow blending did not affect the dispersed-phase size of the $2 \%$ Aerosil 200 in 15\% PC/SAN blend, which is also the blend for which the nanosilica had an effect on the coarse blend size. Therefore, it appears that the two grades of nanosilica behave much differently in the $15 \%$ PC/SAN, even though the TEM images in Figure 4-56 show similar levels of mixing. Additionally, Figure 4-58 shows that multiple 
passes through the stacked orifice dies was not advantageous in affecting the dispersed polymer phase morphology.

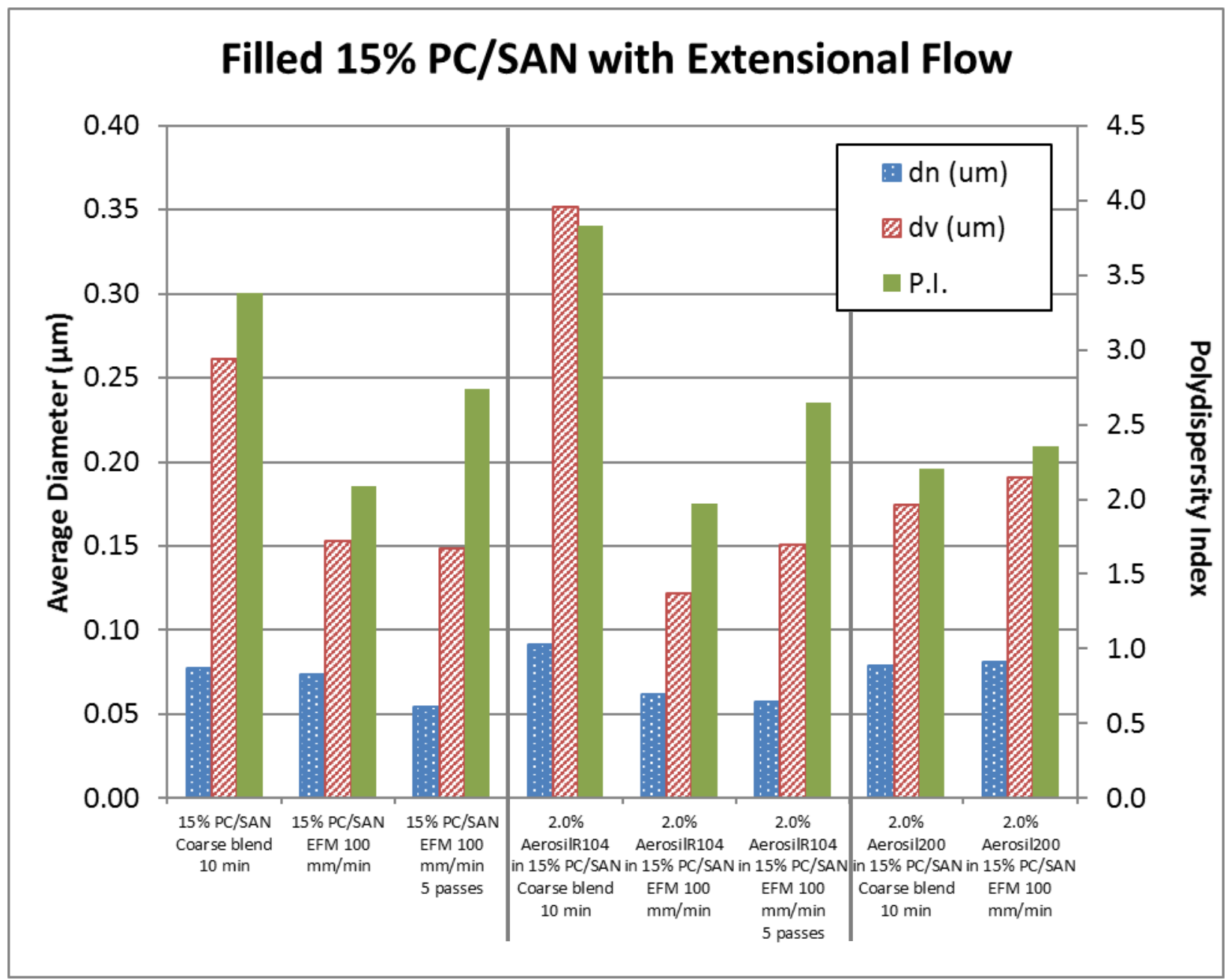

Figure 4-58: Morphology of nanosilica-filled 15\% PC/SAN with extensional flow. Extensional flow was through stacked orifice dies $(\varepsilon=6.5 \& 6.0)$ at $100 \mathrm{~mm} / \mathrm{min}$ piston speed $(Q=17$ $\mathrm{cm}^{3} / \mathrm{min}$ ) with multiple passes as noted. 


\subsubsection{Nano-Filled PS/LLDPE}

\subsubsection{Thermodynamics of Nano-Filled PS/LLDPE}

As discussed in Section 4.1.2.2, the surface properties of the PS and LLDPE phases and the interfacial tension between them depend strongly on the methods used to measure or calculate them. Figure 4-59 shows the wettability parameter for nanoparticles of given surface tensions and surface polarities. The PS/LLDPE interfacial tension used is $4.4 \mathrm{mN} / \mathrm{m}$, calculated using the harmonic mean equation on the phase surface properties from Wu 1982 [52], as given in Table 4-1. As should be expected, nanoparticles with high surface polarity and medium-to-high total surface tension are predicted to localize in the more polar PS phase.

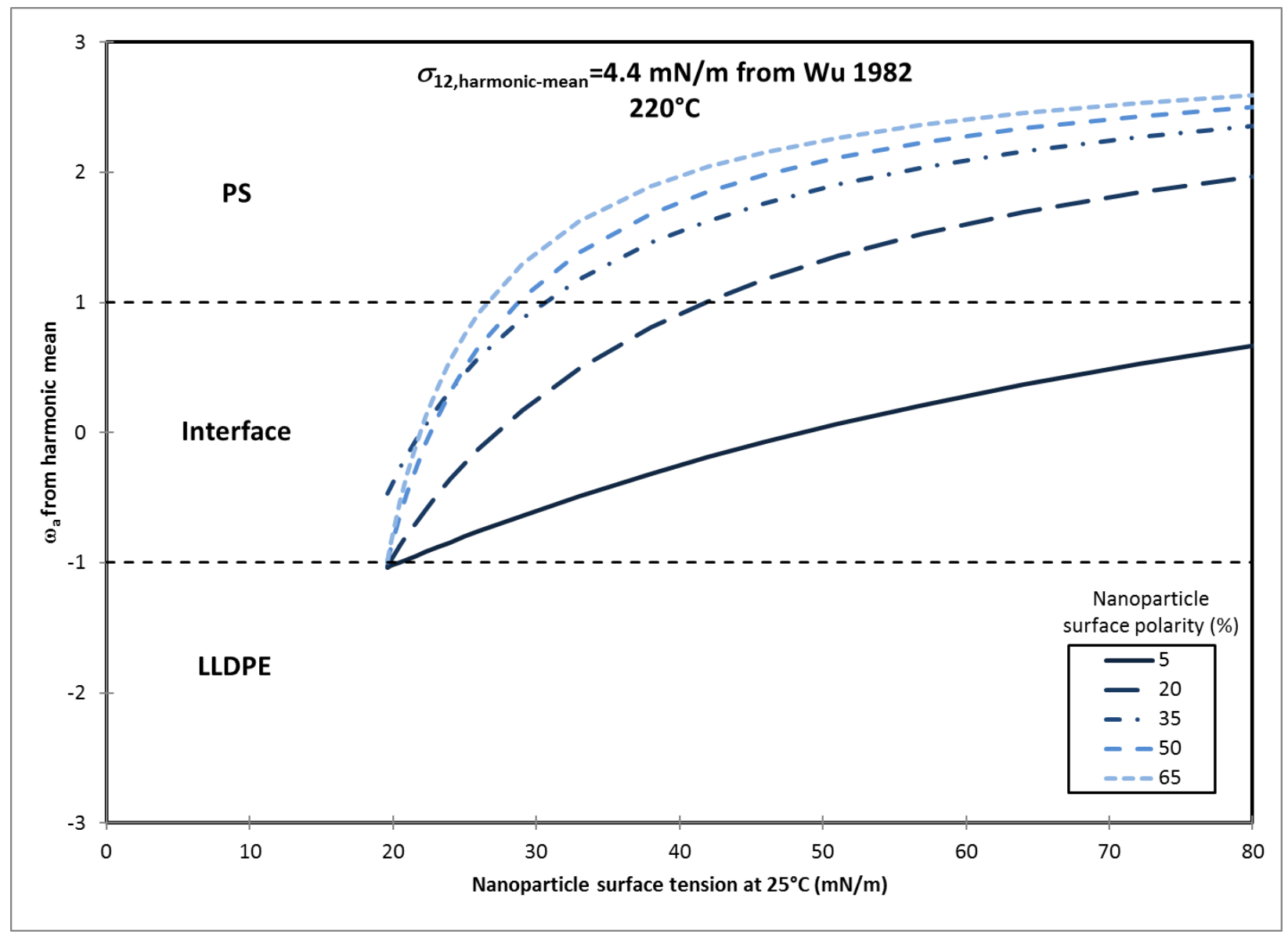

Figure 4-59: Wettability parameter of nanoparticles with various surfaces in PS/LLDPE 


\subsubsection{Nano-Filled PS/LLDPE Coarse Blends}

TEM images of the one step-mixed nano-filled PS/LLDPE coarse blends are shown in Figure 4-60. The contrast between the two polymer phases was greatly enhanced by adjusting the focus of the microscope. As predicted by the wettability parameter plot in Figure 4-59, the hydrophilic nanosilica selectively localizes in the PS dispersed phase. Interestingly, the hydrophobic Aerosil R104 also preferentially localizes in the PS phase. This implies that the hydrophobic coating is either not very hydrophobic, does not cover the surface of the nanoparticles well, or that other effects dominate the behavior of the ternary blend. For example, in one-step mixing in which all three phases are added to the internal mixer simultaneously, PS likely softens before LLDPE and therefore is the first polymer phase to wet the surface of the nanosilica, including the interparticle pores present in the tightly bound aggregates of nanosilica. Also interesting in the TEM images is that the dispersed PS drops seem to have higher concentrations of nanosilica near the radius of the drops, and the nanosilica does appear to modify the PS/LLDPE interface in addition to localizing in the bulk PS phase.

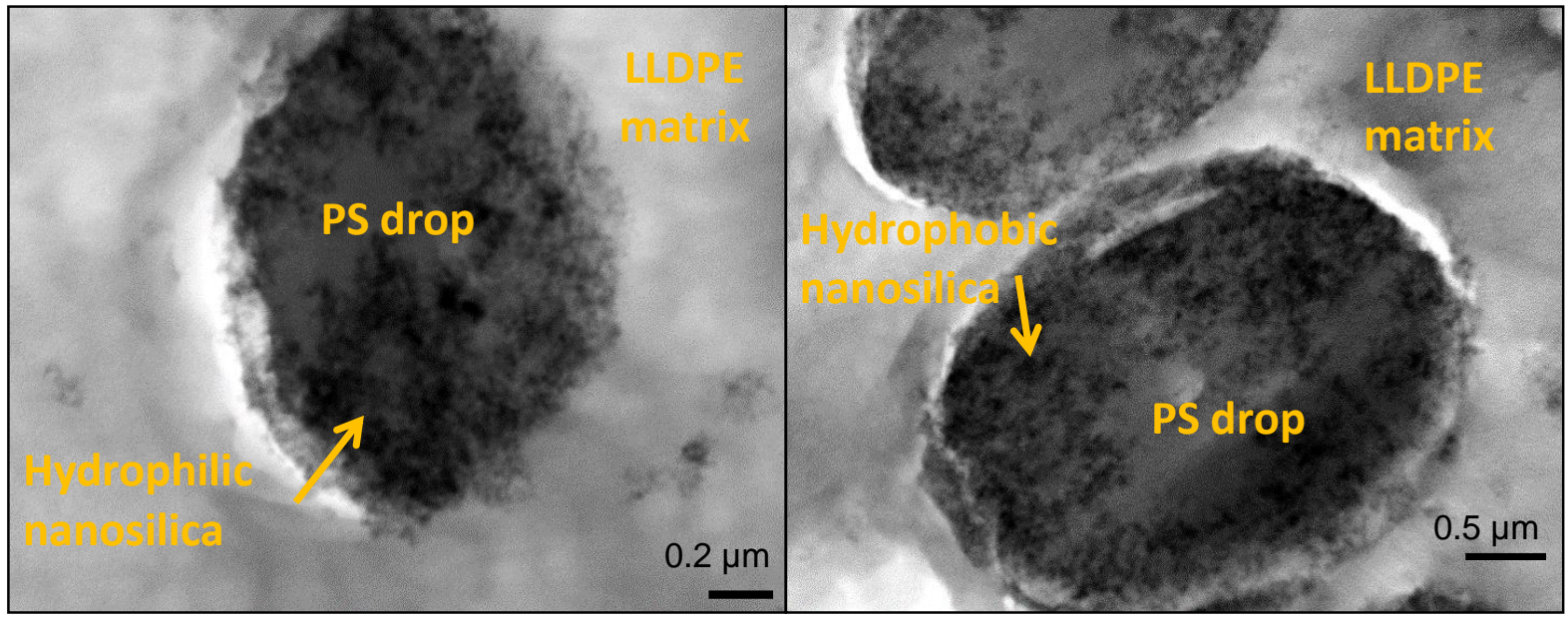

Figure 4-60: Representative TEM images of nano-filled PS/LLDPE coarse blends. One stepmixed blends: Left - 2.9\% Aerosil 200 in 15\% PS/LLDPE and Right - 2.9\% Aerosil R104 in 15\% PS/LLDPE. 


\subsubsection{Extensional Flow with Nanoparticle Stabilization in PS/LLDPE}

The results of extensional flow blending using the hemisphere-in-cone die with 0.002 in (0.051 mm) spacer on the morphology of unfilled and 2.9\% Aerosil R104-filled 15\% PS/LLDPE are given in Figure 4-61. Incorporation of the hydrophobic nanosilica in this blend resulted in a coarse blend with much finer morphology compared to the unfilled blend, and after extensional flow blending, both $d_{\mathrm{n}}$ and $d_{\mathrm{v}}$ decreased further for the nano-filled blend, whereas they both increased slightly for the unfilled blend. The nanosilica preferentially localizing in the dispersed phase, along with significant modification of the polymer-polymer interface, did prove to be useful in both shear flow-dominated and extensional flow-dominated processing.

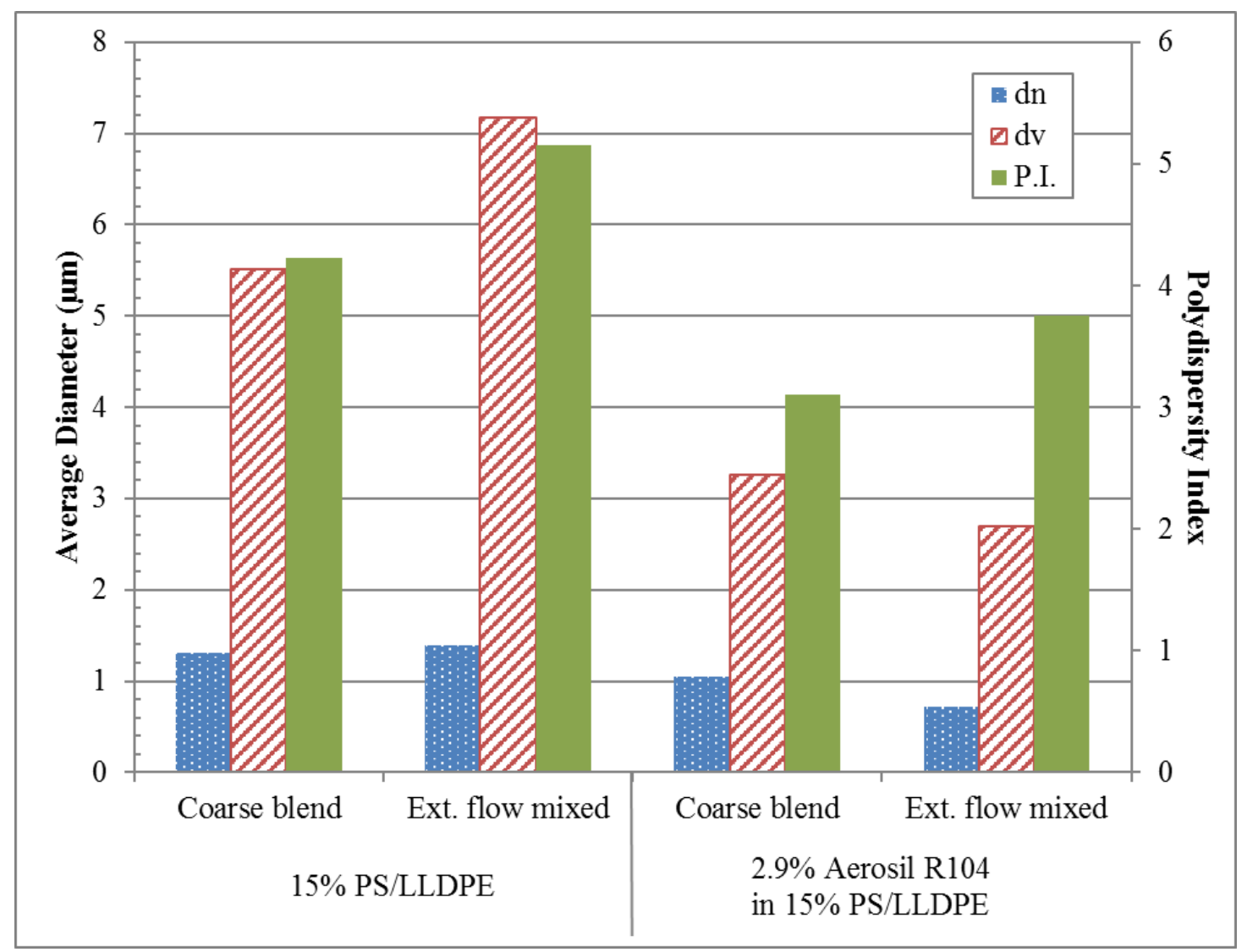

Figure 4-61: Extensional flow blending with nanoparticle stabilization of PS/LLDPE.

Extensional flow through the hemisphere-in-cone die with 0.002 in $(0.051 \mathrm{~mm})$ spacer. 


\subsubsection{Nano-Filled HDPE/PS}

\subsubsection{Thermodynamics of Nano-Filled HDPE/PS}

The surface tensions of and interfacial tension between HDPE and PS were discussed in Section 4.1.3.2 and were presented in Table 4-8 and Table 4-9. The challenge within this work is finding an appropriate method for measuring the surface properties of the constituent materials that works well for the different material forms present. A goniometer to visually observe the contact angle between a liquid and a smooth, flat surface of the polymer of interest is commonly used to measure the solid-phase polymer surface properties. This method does not work well for nanomaterials in fine powder form, though, because the material must be pressed into a cake, which inevitably has surface roughness that affects the measured contact angle. The surface properties of powders may be measured by observing capillary action of a liquid wetting the powder in a tube, but to accomplish this for the polymer, a fine powder must first be created. For these reasons, there is a large degree of between-lab variation in surface tension data, and to be robust in this work, several different sources were used both for the surface properties of the constituent materials and for the interfacial properties between the constituents.

The wettability parameter for a single, isolated, non-porous, generalized nanoparticle of a given surface tension at room temperature and surface polarity as well as that for each of the nanoparticles considered in this work was calculated and plotted using three different sets of surface and interfacial tensions. These plots are given in Figure 4-62, Figure 4-63, and Figure 4-64 for HDPE/PS interfacial tensions $\sigma_{12}$ of $0.44 \mathrm{mN} / \mathrm{m}, 4.46 \mathrm{mN} / \mathrm{m}$, and $5.46 \mathrm{mN} / \mathrm{m}$, respectively. Furthermore, the HDPE and PS surface tensions and surface polarities were the average values from the literature for the first and the last plot and were the values from $\mathrm{Wu}$ 1982 [52] for the middle plot. 


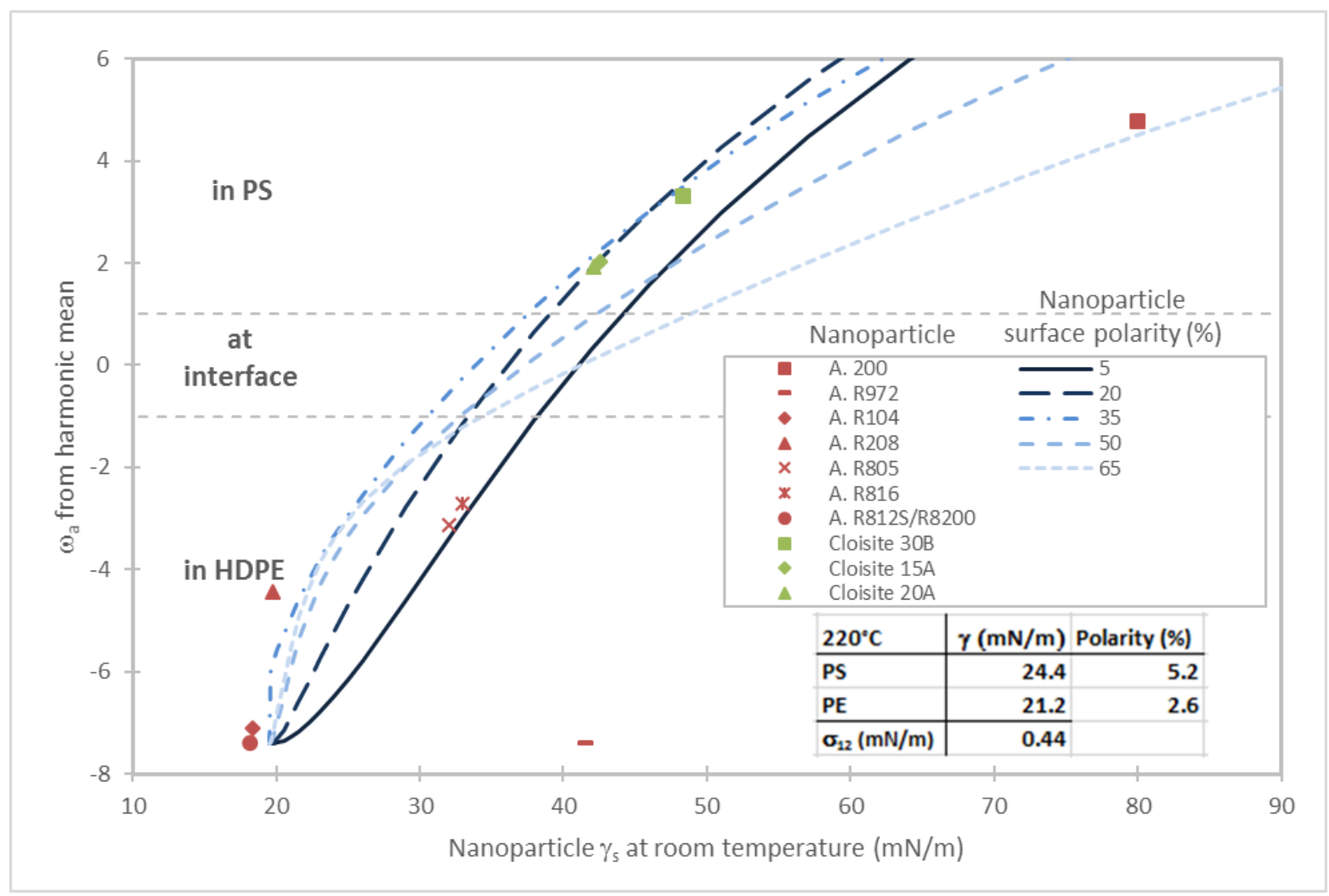

Figure 4-62: Wettability parameter plot for $\sigma_{12, \mathrm{HDPE} / \mathrm{PS}}=0.44 \mathrm{mN} / \mathrm{m}$

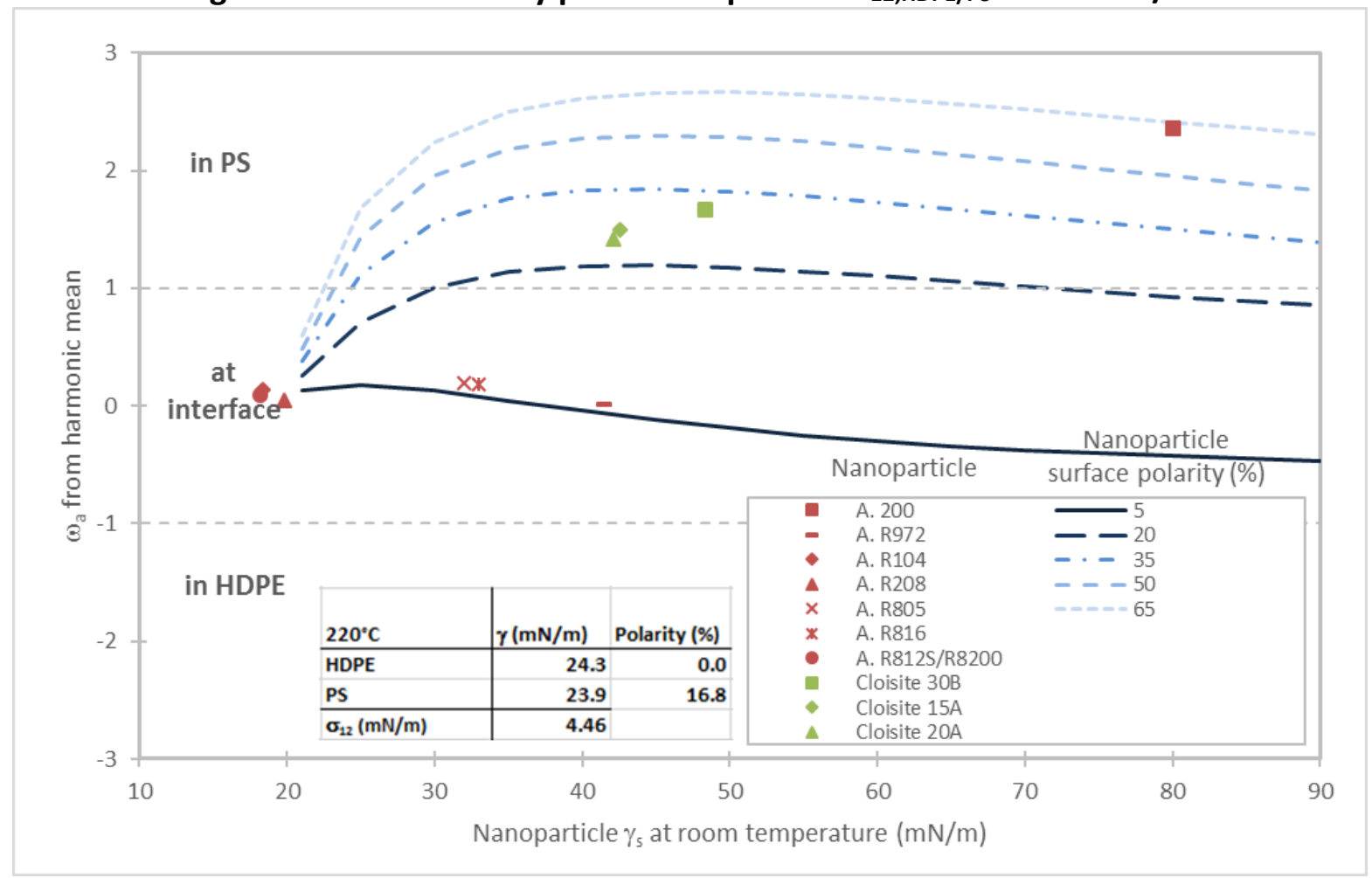

Figure 4-63: Wettability parameter plot for $\sigma_{12, \mathrm{HDPE} / \mathrm{PS}}=4.46 \mathrm{mN} / \mathrm{m}$ 


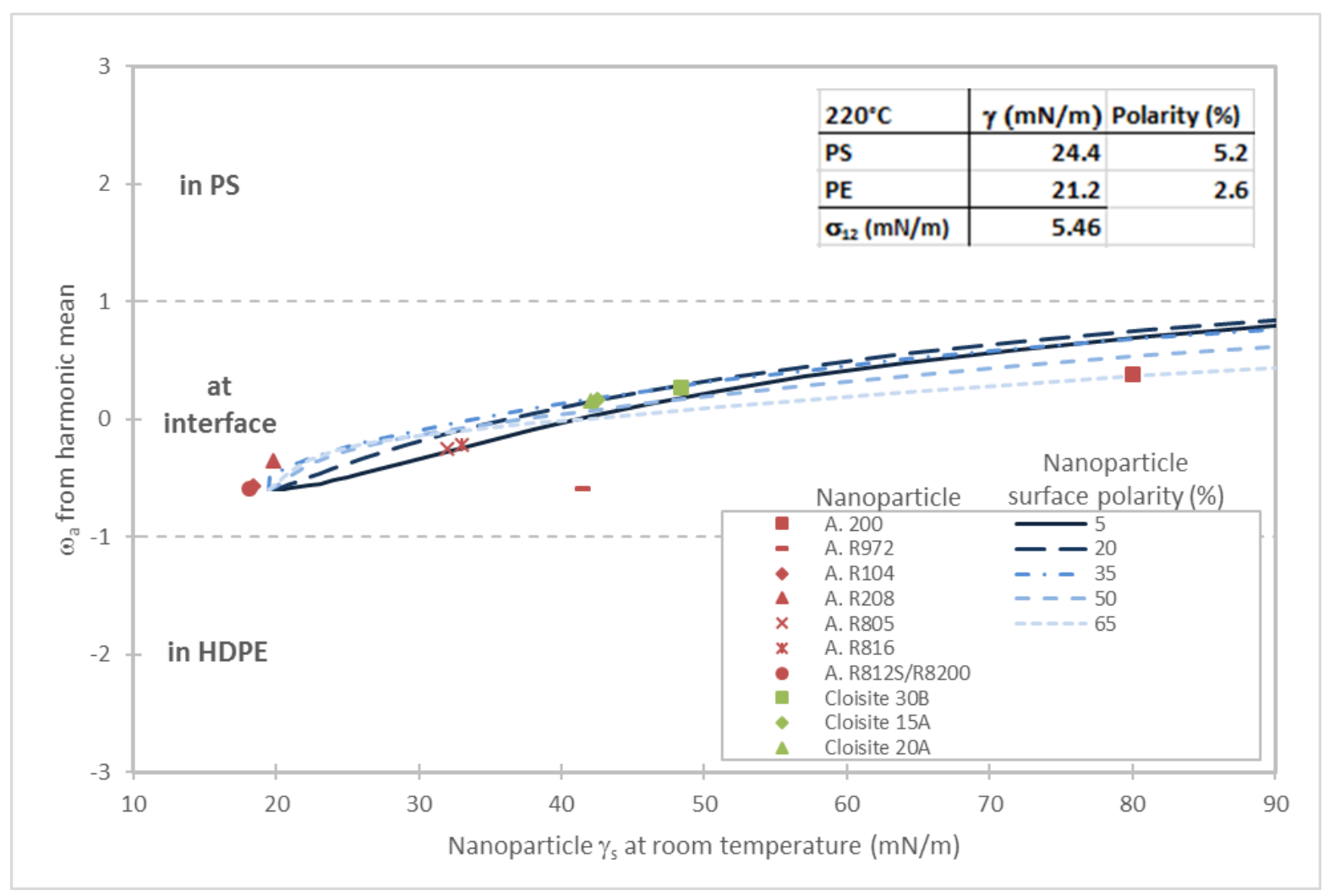

Figure 4-64: Wettability parameter plot for $\sigma_{12, \mathrm{HDPE} / \mathrm{PS}}=5.46 \mathrm{mN} / \mathrm{m}$

In Figure 4-62, when the interfacial tension is small and the difference in surface polarity between the constituent polymers is small, none of the nanoparticles considered are predicted to thermodynamically localize at the interface. The driving force to do so is quite small, especially because the interfacial tension between the polymers is so small in this scenario. In Figure 4-63, both the interfacial tension and the difference in surface polarity are considerably larger, and all of the surface-treated nanosilica grades are predicted to localize at the interface. Though, the hydrophilic nanofillers, Aerosil 200 and the Montmorillonite nanoclay grades, Cloisite from Southern Clay Products, are predicted to localize in the more polar PS phase. Finally, in Figure 4-64, the interfacial tension is larger yet and the difference in surface polarity between the polymers is small. For this scenario, all of the nanofillers are predicted to localize at the interface. The large interfacial tension between the polymers creates a large driving force for surface modification, and the similarities in surface polarities between the phases 
ensures that the nanofiller interacts favorably with both phases while at the interface. These are the conditions that promote a third phase to modify the interface.

\subsubsection{Nano-Filled HDPE/PS Coarse Blends}

The coarse blend mixing approach using the internal mixer was studied further for the nano-filled HDPE/PS blends. The order of mixing was varied, and the following two approaches were used: 1) one-step mixing, in which all three phases were mixed in one melt-processing step, and 2) two-step mixing, in which the nanosilica was pre-mixed into the dispersed HDPE phase first to create a masterbatch and a subsequent melt-processing step was taken to mix the masterbatch with the PS matrix phase. These are described in Section 3.3.1.2.

\subsection{One-Step Mixing}

In one-step mixing, the HDPE and PS phases were pre-melted for about 3 min before addition of the nanosilica. This approach was taken rather than adding all three phases at one time to counteract the effect of one polymer melting before the other and wetting the nanoparticles first. In this way, the thermodynamics associated with the interaction between the nanoparticles and each of the polymer phases was given a better opportunity to dictate the behavior of the system. After the nanosilica was added, the three phases were mixed for at least $5 \mathrm{~min}$ and sometimes up to $45 \mathrm{~min}$ to observe whether the nanoparticles would migrate from one phase to another. The resulting phase localization of the nanoparticles was observed using scanning electron microscopy (SEM) and energy dispersive $x$-ray spectroscopy (EDS) mapping. The size of the nanoparticles at about $12 \mathrm{~nm}$ is about the limit to the resolution of SEM, and in practice, the gold-palladium electrically conductive surface sputter-coated onto the samples creates a surface roughness on the same scale. Therefore, direct observation of the nanoparticles with SEM is difficult, but surface roughness may be observed if the nanoparticles localize at the polymer-polymer interface. Likewise, the spatial resolution of EDS and EDS mapping is much coarser than the size of the nanoparticles. Conclusive information using EDS alone was seldom gained, except when the nanosilica localized exclusively in the dispersed 
phase, in which case, the EDS mapping can confirm that Si atoms are not detected throughout the matrix phase.

The results of one step-mixing of each of the nanosilica grades into $5 \% \mathrm{HDPE} / \mathrm{PS}$ are given in Table 4-18. All of the one step-mixed blends showed nanosilica selectively localized in the PS matrix phase, regardless of the surface properties of the nanoparticle or mixing time. This is corroborated by the representative EDS map in Figure 4-65, in which the magenta pixels corresponding to Si atoms and the green pixels corresponding to O atoms, both of which only derive from the nanosilica, $\mathrm{SiO}_{2}$, are evenly distributed throughout the image. Due to the poor resolution of EDS mapping, Si and O atoms appearing in HDPE dispersed-phase drops do not necessarily mean that nanosilica was localized in the HDPE phase because the source of the emitted x-ray could have been in the PS matrix below the drop and travelled through the drop toward the EDS detector. The EDS mapping is best used to determine if the nanosilica localized exclusively in the dispersed phase, which was not observed for the one step-mixed coarse blends.

TEM images were also obtained of one step-mixed 4\% hydrophilic Aerosil 200 in 5\% HDPE/PS and 4\% hydrophobic Aerosil R104 in 5\% HDPE/PS, shown in Figure 4-66. The nanosilica exclusively localized in the PS phase for both nano-filled blends. Also observed in the blend with the hydrophilic nanosilica was a large agglomerate of nanosilica which was not broken up after processing in the internal mixer. In contrast, the hydrophobic nanosilica showed better dispersion. This is expected to be due to the larger surface energy of the hydrophilic nanomaterial which creates a larger driving force for agglomeration and even flocculation.

The hypothesis to why the nanosilica grades exclusively localized in the PS phase, even though the wettability parameter calculations predicted at least a subset of them to selectively localize at the interface, is that kinetic effects far outweigh thermodynamic effects in the blends. The very large viscosity mismatch, along with the relatively low concentration of the dispersed phase, likely influence the localization of the nanofiller more than the thermodynamic driving force for the nanofiller's localization. Furthermore, the aggregated nature of the nanofiller phase creates inter-particle porosity, which further complicates the 
situation. The first molten polymer to wet these inter-particle pores likely remains there due to capillary action and essentially becomes part of the aggregate particle. Since the concentration of PS in the blends is so high, 95 wt\%, PS is likely the first to wet the nanoparticles and effectively modifies the surface of the nanoparticle aggregates. Future work would be required to test and potentially implement solutions for this phenomenon.

The effect of one step-mixing on the coarse blend dispersed polymer phase morphology will be discussed in the next section along with two step-mixing. 
Table 4-18: Nanosilica localization in one step-mixed coarse blends. Observed using SEM and EDS.

\begin{tabular}{|c|c|c|c|}
\hline Aerosil grade & $\begin{array}{l}\text { Nanosilica concentration } \\
\text { (wt } \%)\end{array}$ & $\begin{array}{l}\text { Mixing time } \\
\text { (min) }\end{array}$ & Nanosilica localization \\
\hline \multirow{3}{*}{200} & 2 & 5 & PS \\
\hline & 2 & 31 & $\begin{array}{l}\text { PS and very few solid } \\
\text { aggregates at } \\
\text { interface }\end{array}$ \\
\hline & 2 & 45 & PS \\
\hline R974 & 1 & 30 & $\begin{array}{l}\text { PS and a few solid } \\
\text { aggregates at } \\
\text { interface }\end{array}$ \\
\hline \multirow{2}{*}{ R816 } & 1 & 28 & PS \\
\hline & 2 & 5 & PS \\
\hline R104 & 2 & 5 & PS \\
\hline \multirow{2}{*}{ R812S } & 1 & 30 & PS \\
\hline & 2 & 5 & PS \\
\hline \multirow[b]{2}{*}{ R8200 } & 1 & 45 & PS \\
\hline & 2 & 5 & $\begin{array}{l}\text { PS and very few solid } \\
\text { aggregates at } \\
\text { interface }\end{array}$ \\
\hline \multirow{3}{*}{ R208 } & 0.5 & 6 & PS \\
\hline & 2 & 5 & PS \\
\hline & 4 & 6 & PS \\
\hline
\end{tabular}




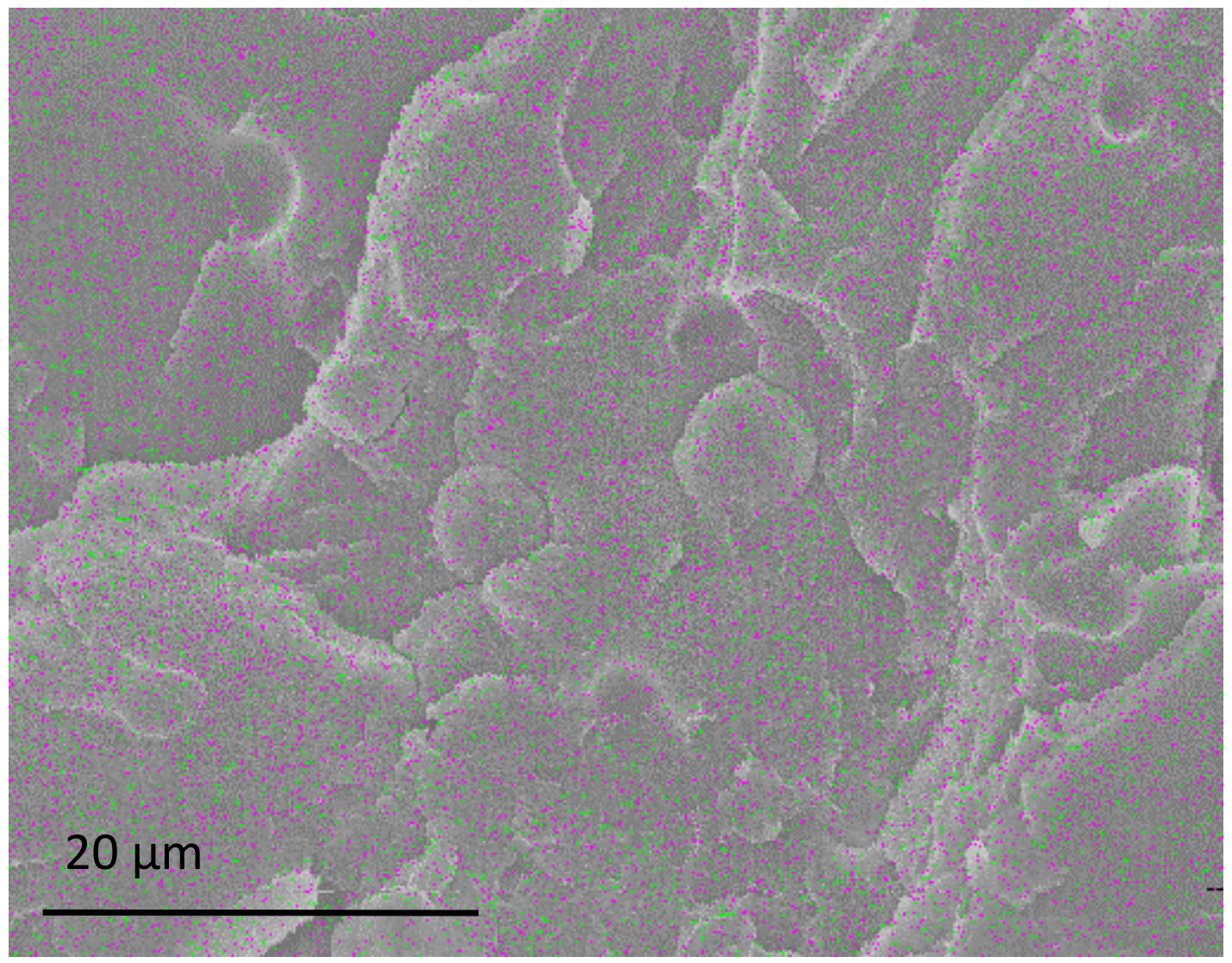

Figure 4-65: Representative EDS map of one-step mixed nano-filled blend. Magenta pixels correspond to $\mathrm{Si}$ atoms, and green pixels correspond to $\mathrm{O}$ atoms.

\section{Hydrophilic nanosilica (Aerosil 200) Hydrophobic nanosilica (Aerosil R104)}

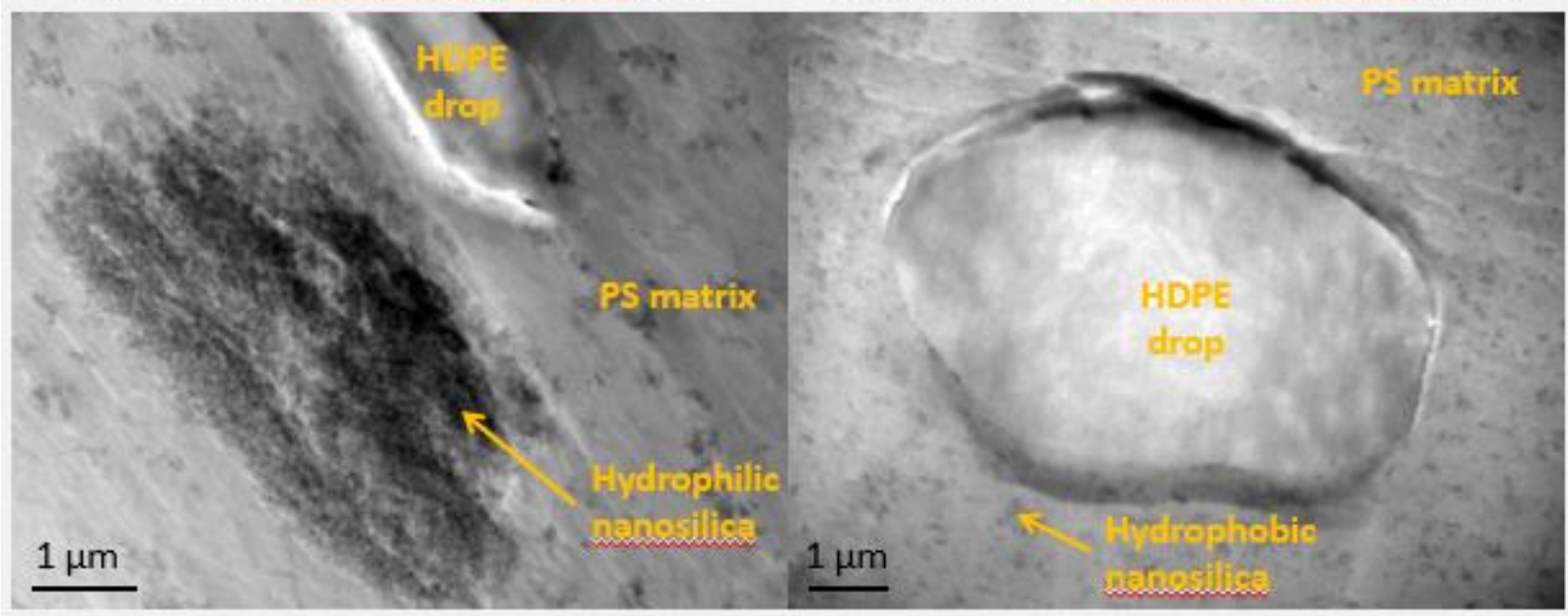

Figure 4-66: Representative TEM images of one step-mixed coarse blends. The dark phase corresponds to nanosilica agglomerates or aggregates, and the HDPE drops are denoted within the PS matrix. 


\subsection{Two-Step Mixing}

The second approach taken to mixing the nanofiller into the binary polymer blend was a two step-mixing strategy. Because the results of one-step mixing showed that every grade localized in the PS phase, the two step-mixing approach was to pre-mix the nanosilica into the HDPE phase in a masterbatch, then in a second melt-processing step, mix the masterbatch with the PS matrix material. The first step typically used a mixing times of 5-10 min, and the second step mixed for at least 6 min and up to $45 \mathrm{~min}$, again to inspect whether the nanosilica would migrate between phases. The resulting phase localization was again observed using SEM and EDS, and the results are given in Table 4-19.

All of the nanosilica grades used in the two step-mixing approach remained in the HDPE phase, regardless of the surface properties of the nanofiller or mixing time. A representative EDS map is shown in Figure 4-67, in which magenta pixels corresponding to Si atoms are exclusively associated with dispersed-phase HDPE drops. The gradient of magenta pixels that goes into the PS matrix phase is an indication of the poor spatial resolution of EDS and likely does not imply that the nanosilica was actually migrating out of the HDPE phase. Additionally, very large nanosilica/HDPE domains were observed for the two step-mixed coarse blends. These are shown in Figure 4-68 for both the hydrophilic Aerosil 200-filled blend and the hydrophobic Aerosil R104-filled blend. Each of those blends were formulated to produce a final three-phase blend with $2 \%$ nanosilica, but to achieve this in a masterbatch in the dispersed phase polymer that is only loaded at $5 \%$ in the final blend, a high concentration of nanosilica is needed in the masterbatch. These are given in Table 3-3 in Section 3.3.1.2. This effectively increased the viscosity ratio of the blend and the dispersed-phase elasticity, making dispersive blending even more difficult. Therefore, the large domains several hundred microns in size in the two step-mixed coarse blends are not surprising after processing in the internal mixer which employs predominantly shear flow to impart mixing onto the materials. 
Table 4-19: Nanosilica localization in two step-mixed coarse blends. Observed by SEM and EDS.

\begin{tabular}{|c|c|c|c|}
\hline $\begin{array}{l}\text { Aerosil } \\
\text { grade }\end{array}$ & $\begin{array}{l}\text { Final nanosilica } \\
\text { concentration } \\
\text { (wt\%) }\end{array}$ & $\begin{array}{l}\text { Mixing time } \\
\text { (min) }\end{array}$ & Nanosilica localization \\
\hline \multirow{5}{*}{200} & 0.1 & 6.5 & HDPE \\
\hline & 1 & 45 & HDPE \\
\hline & 2 & 10 & HDPE \\
\hline & 2 & 30 & $\begin{array}{l}\text { HDPE with some surface } \\
\text { roughness on HDPE drops }\end{array}$ \\
\hline & 2 & 47 & HDPE \\
\hline R974 & - & - & - \\
\hline R816 & 1 & 30 & HDPE \\
\hline R104 & 2 & 10 & HDPE \\
\hline R812S & - & - & - \\
\hline R8200 & - & - & - \\
\hline R208 & - & - & - \\
\hline
\end{tabular}




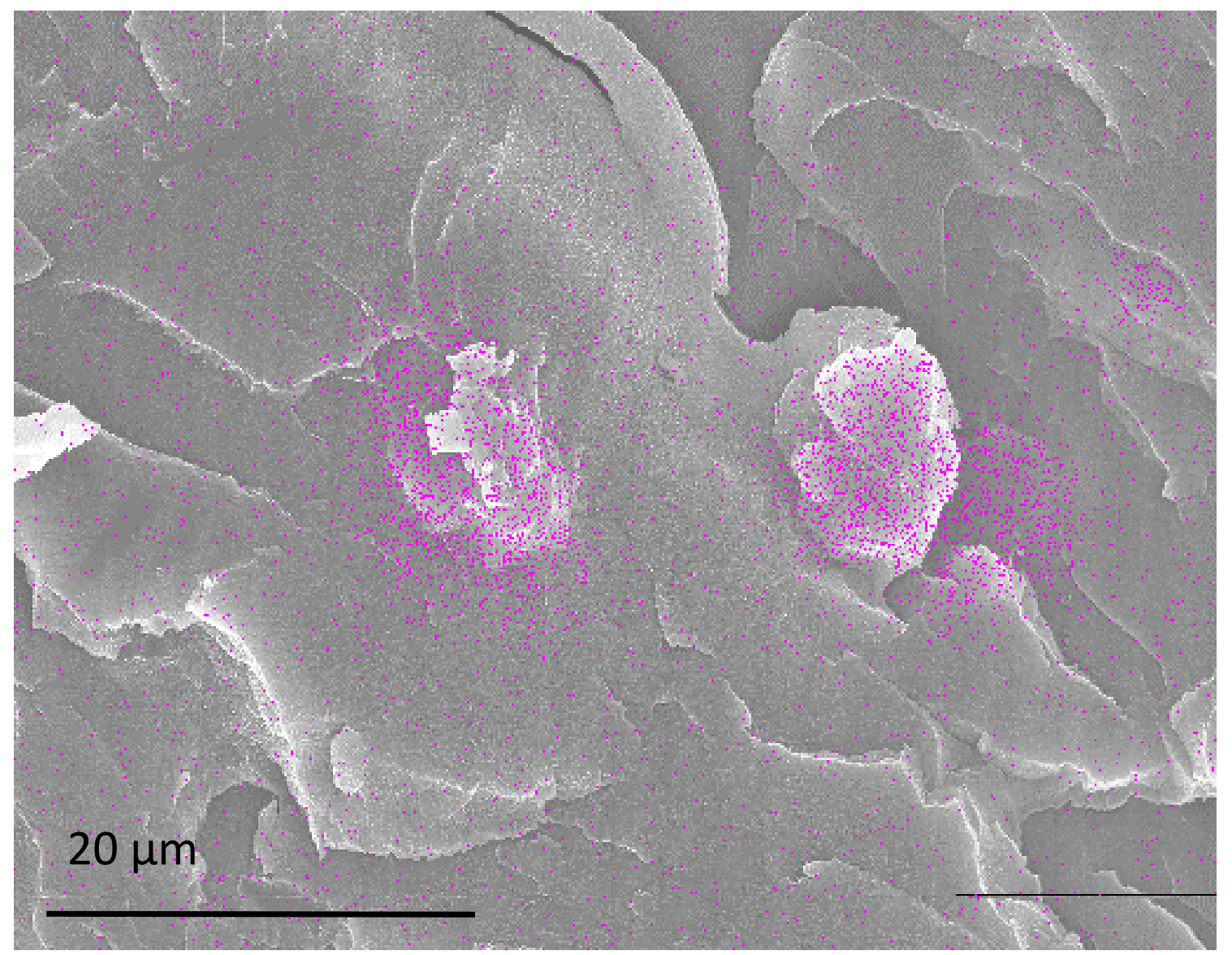

Figure 4-67: Representative EDS map of two-step mixed nano-filled blend. Magenta pixels correspond to Si atoms. 

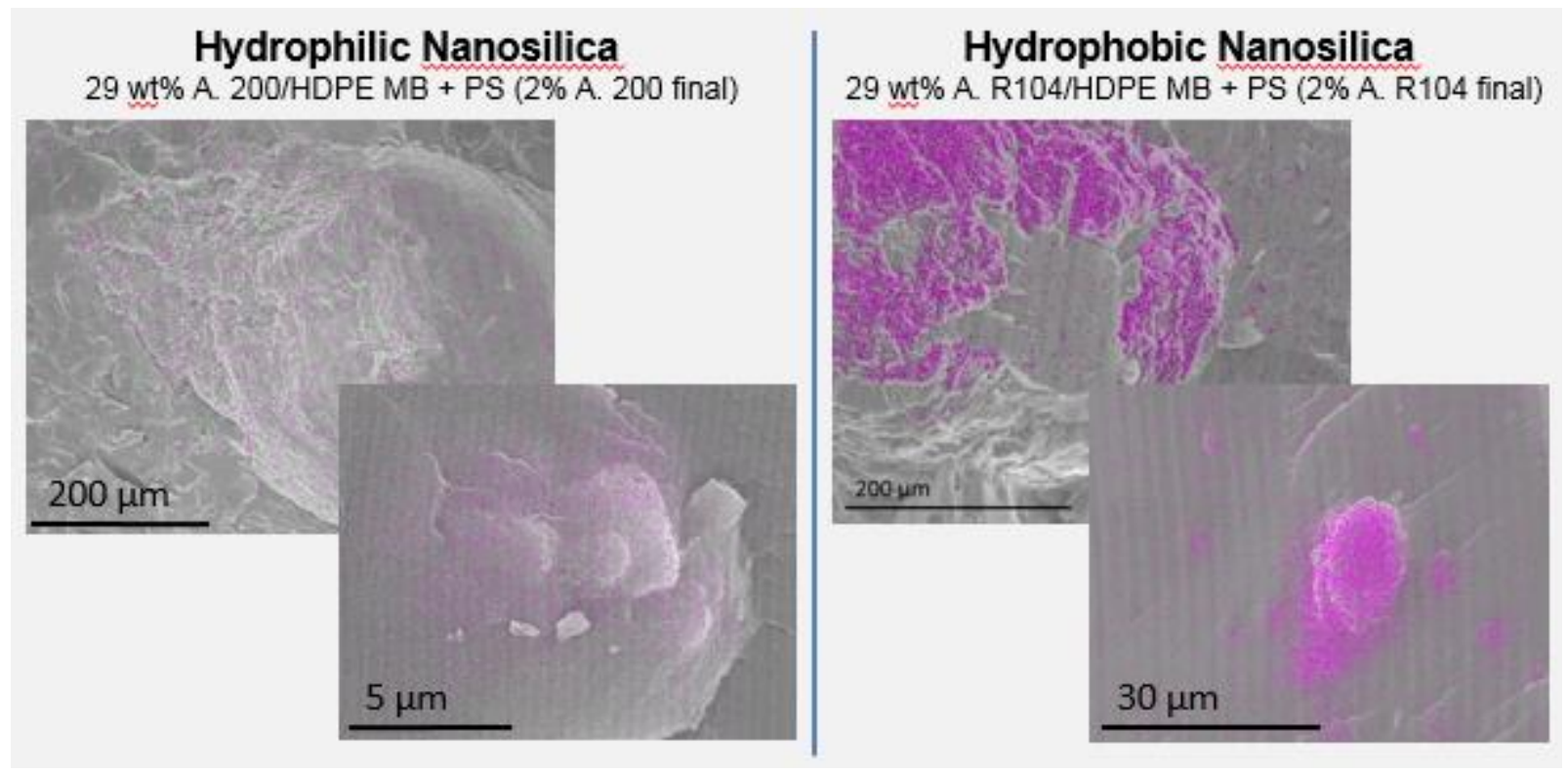

Figure 4-68: Comparison of EDS maps of two-step mixed nano-filled blends. Magenta pixels correspond to Si atoms.

The effects of the two different coarse blend mixing approaches on the dispersed polymer phase morphology are shown in Figure 4-69. The one step-mixed blends all yielded decreases in $d_{v}$, by a factor of about 2, compared to the unfilled coarse blend. Two blends with Aerosil R208, the PDMS-coated nanosilica, were processed with different loadings of nanosilica, $0.5 \%$ and $4 \%$, and they each produced similar morphologies. This implies that just $0.5 \%$ nanosilica was sufficient at least for the Aerosil R208 grade. Those two blends did not show a decrease in $d_{\mathrm{n}}$ compared to the unfilled coarse blend, therefore the size distribution, P.I. showed a significant reduction. The hydrophilic Aerosil 200 and hydrophobic Aerosil R104 yielded very similar coarse blends, with a decreased $d_{\mathrm{n}}$ compared to that of the unfilled blend. The reduction in dispersed polymer phase size, especially that of $d_{v}$, using one step-mixing of a nanofiller into the blends is likely due to disruptions of the coalescence process. The viscosity of the matrix phase and of the blend as a whole was not significantly affected by the loading of the nanoparticles in the concentrations used, so the nanofiller likely did not make a significant impact on drop breakup. However, because the solid nanoparticles are evenly distributed throughout the matrix phase, they may serve as physical barriers to coalescence, and they likely 
affect film drainage of the matrix material between colliding dispersed-phase drops, which is the limiting step of the coalescence process.

The results of two step-mixing on the coarse blend morphology are starkly different from those of the one step-mixed blends. The coarse blend was prepared by two-step mixing of Aerosil 200 in 5\% HDPE/PS, first mixing 9.1\% nanosilica into the HDPE phase, then mixing that with PS to create a $0.5 \%$ loading in the final blend. The dispersed polymer phase morphology was about 4.5-5.0 times coarser than that of the unfilled coarse blend, with respect to both $d_{\mathrm{n}}$ and $d_{\mathrm{v}}$, therefore, interestingly, the P.I. was comparable to that of the unfilled blend. This high concentration of solid nanofiller into the HDPE in the first mixing step causes the already viscous and elastic HDPE phase to become even more so. The dynamic rheology of several of the materials and blends of this work is given in Figure 4-70 and Figure 4-71, showing the complex viscosity vs. frequency curves and the storage and loss moduli vs. frequency, respectively.

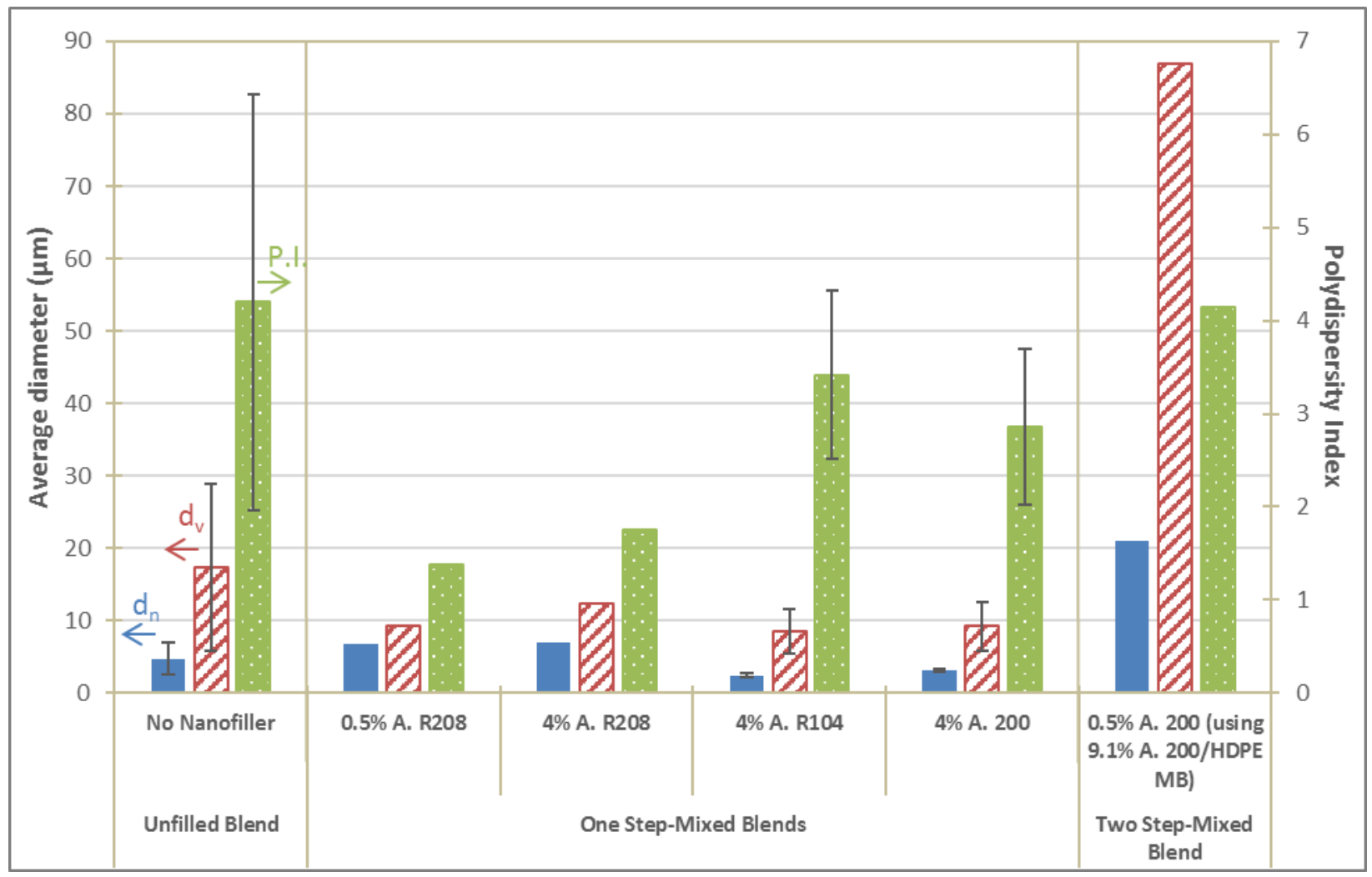

Figure 4-69: Effect of mixing procedure on coarse blend morphology. 


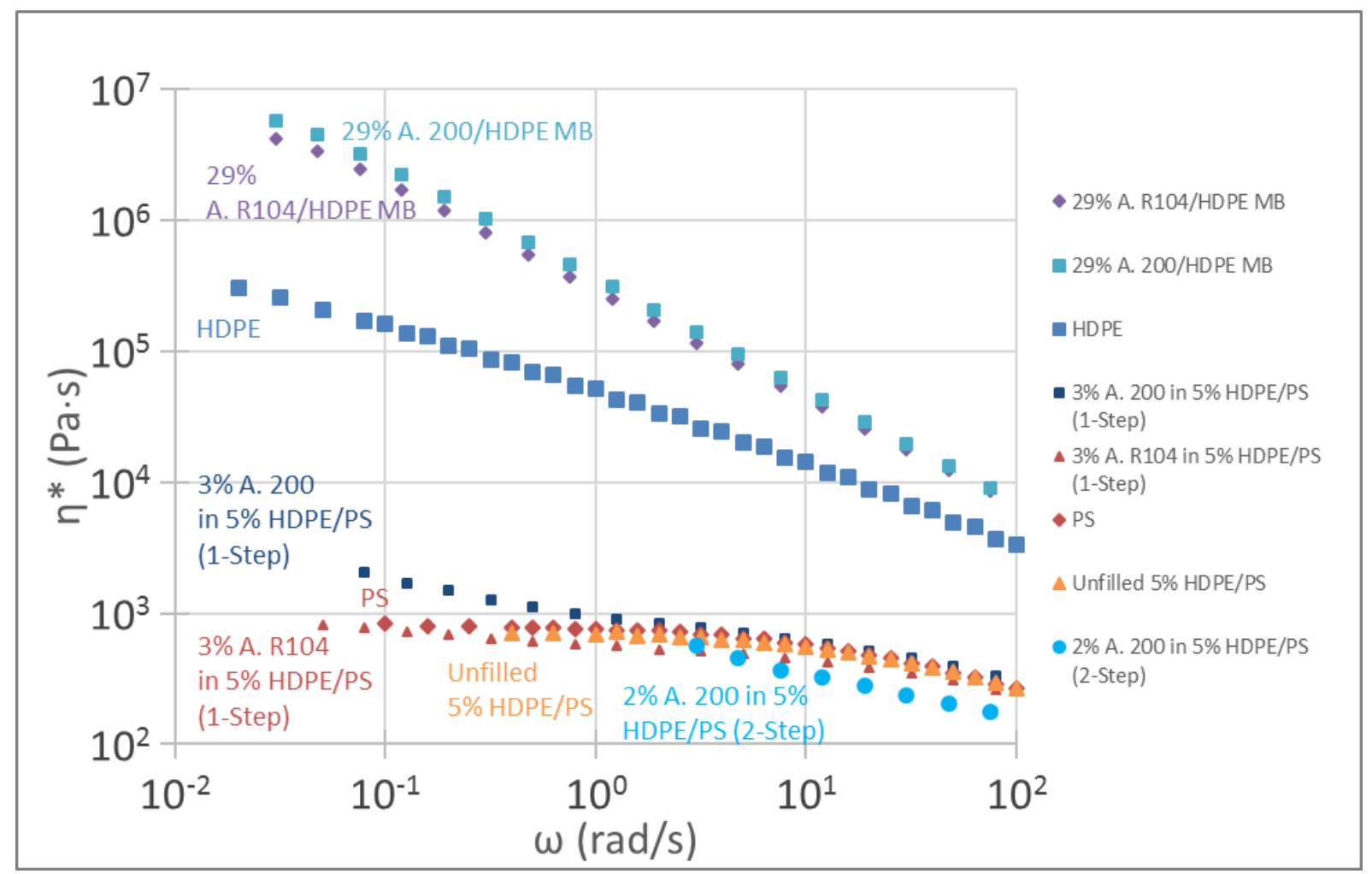

Figure 4-70: Complex shear viscosity vs. frequency for nano-filled blends.

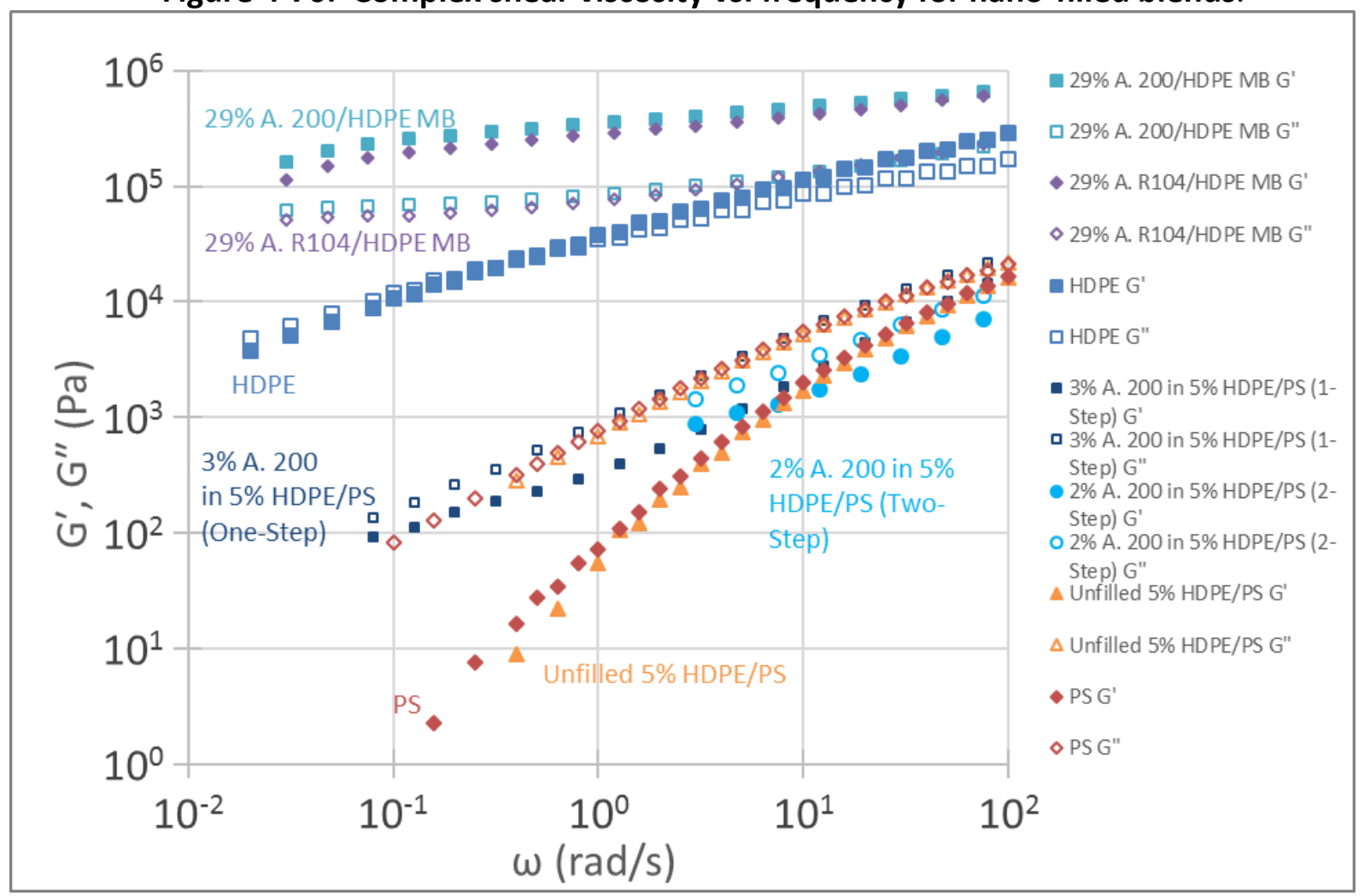

Figure 4-71: Storage and loss modulus vs. frequency for nano-filled blends. 
The viscosities of the $29 \%$ nanosilica/HDPE masterbatches, which were used to produce final blends of $2 \%$ nanosilica in 5\% HDPE/PS, were about 10 times greater than that of the unfilled HDPE, thereby increasing the viscosity by a factor of 10 at low shear rates. For these masterbatch-filled blends, the viscosity ratio is on the order of 1000 , making dispersive mixing with shear flow-dominant processing impossible. Furthermore, the storage modulus, $G^{\prime}$, of the masterbatch increases by more than an order of magnitude and the crossover point where $G^{\prime \prime}$ crosses over $G^{\prime}$ is no longer observed. Both of these effects on the rheology indicate that the elasticity of the dispersed phase also increased dramatically with the high loading of solids.

The other materials and blends in Figure 4-70 and Figure 4-71 show that the loading of nanoparticles in the one step-mixed blends and in the final blends did not significantly increase the viscosity or flow modulus values. The behavior of $G^{\prime \prime}$ and $G^{\prime}$ at low frequencies is that associated with the Palierne model for viscoelastic behavior of emulsions where interfacial effects are important [81-82].

\subsubsection{Extensional Flow with Nanoparticle Stabilization}

A representative TEM image of the one step-mixed 4\% Aerosil R104 in 5\% HDPE/PS

blend, the coarse blend of which was shown in Figure 4-66, is shown in Figure 4-72 representing the morphology after extensional flow blending through the converging cone die with 0.330 $\mathrm{mm}$ exit diameter, $\varepsilon=7.1$, at $100 \mathrm{~mm} / \mathrm{min}$, or $17 \mathrm{~cm}^{3} / \mathrm{min}$, and at $220^{\circ} \mathrm{C}$. Extensional flow blending did not appear to significantly affect the level of mixing of the nanosilica aggregates or their phase localization. The hydrophobic nanosilica was still exclusively found in the PS matrix phase. 


\section{Hydrophobic nanosilica (Aerosil R104)}

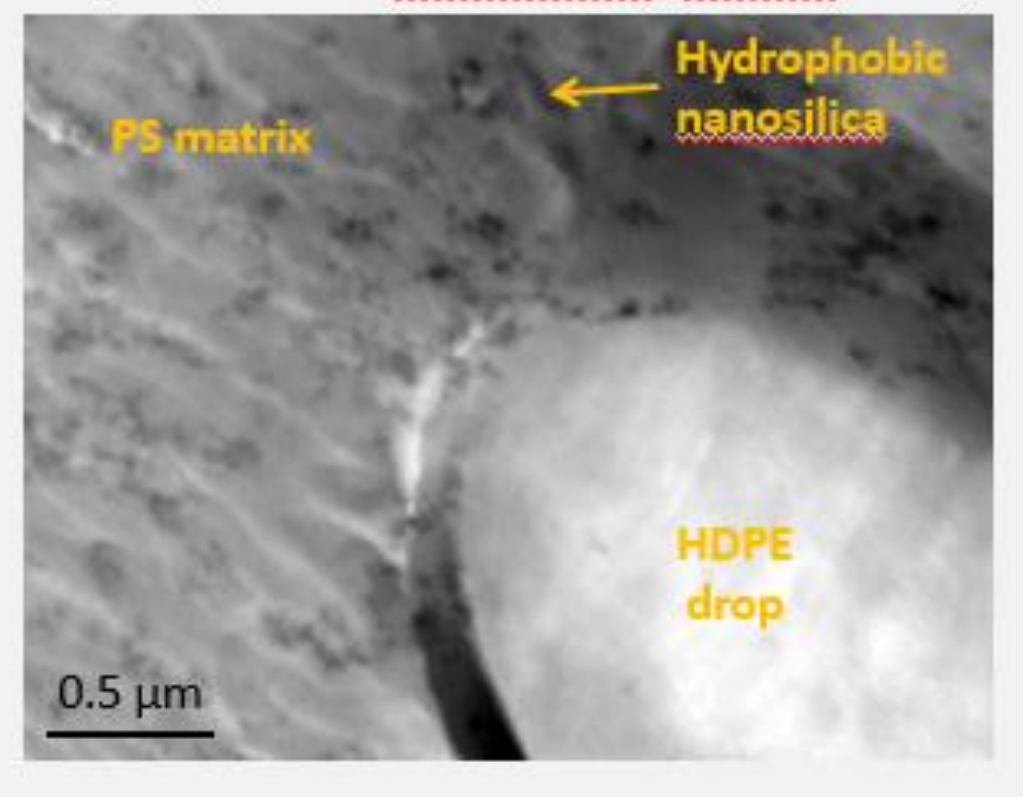

Figure 4-72: Representative TEM image of one step-mixed blend after extensional flow. Extensional flow blending of 4\% Aerosil R104 in 5\% HDPE/PS through the converging cone die with $0.330 \mathrm{~mm}$ exit diameter, $\varepsilon=7.1$, at $100 \mathrm{~mm} / \mathrm{min}$ piston speed, or $17 \mathrm{~cm} / \mathrm{min}$ flow rate, at $220^{\circ} \mathrm{C}$.

The effects of extensional flow blending through the converging cone die with 0.330 $\mathrm{mm}$ exit diameter, $\varepsilon=7.1$, at $100 \mathrm{~mm} / \mathrm{min}$ piston speed, or $17 \mathrm{~cm}^{3} / \mathrm{min}$ flow rate, at $220^{\circ} \mathrm{C}$, on the morphology of one step-mixed nano-filled blends are shown in Figure 4-73. Extensional flow blending effectively decreased $d_{v}$ in most of the one step-mixed blends and slightly decreased $d_{\mathrm{n}}$ in several of the blends. The final morphology after extensional flow blending was almost identical to that of the extensional flow-blended unfilled blend, though. This implies that no coupled effects were observed of using both the incorporation of nanoparticles into the matrix phase and extensional flow blending. The main mechanism by which nanoparticles stabilize the morphology, though, is likely inhibition of coalescence. The extensional flow blending of the unfilled and nano-filled blends was the primary source of mixing to create a fine morphology, and since the extrudate strand was quenched in water, the blend system was never given an opportunity for coalescence to occur. This explains why no significant difference was observed between the morphology of the unfilled extensional flow-blended sample and 
that of the nano-filled extensional flow-blended sample. The incorporation of nanoparticles into these blends would likely affect the preservation of the morphology during secondary melt processes; this is an item that may be further studied in future work.

While no significant effects were observed of the surface properties of the nanofiller on the morphology after extensional flow blending, the loading of nanosilica appeared to influence the morphology development of the nano-filled blends. $0.5 \%$ Aerosil R208 in 5\% HDPE/PS yielded no change in the dispersed polymer phase morphology after extensional flow blending, whereas $4 \%$ loading of the PDMS-coated nanosilica did impart a decrease in both $d_{\mathrm{n}}$ and $d_{\mathrm{v}}$ of the extensional flow-blended sample compared to its coarse blend. No attempt was made to find an optimum loading for effects on morphology after extensional flow blending; this may be reserved for future investigation.

The pressure drop required for extensional flow blending of the one step-mixed nanofilled blends through the converging cone die was about 4 to $21 \%$ higher than that for extensional flow blending of the unfilled blend. This is explained by the minimal effects the loading used in this work for the one step-mixed blends had on the polymer viscosity, as shown in Figure 4-70, and the slight increase in pressure drop may be associated with the slight increase in the storage and loss moduli $G^{\prime}$ and $G^{\prime \prime}$, as given in Figure 4-71. Interestingly some of the one step-mixed nano-filled blends exhibited melt fracture and some did not. This may be an indication that the $17 \mathrm{~cm}^{3} / \mathrm{min}$ flow rate used is very close to the onset of melt fracture, with variations in the material's elastic response likely dictating the exact onset point. 


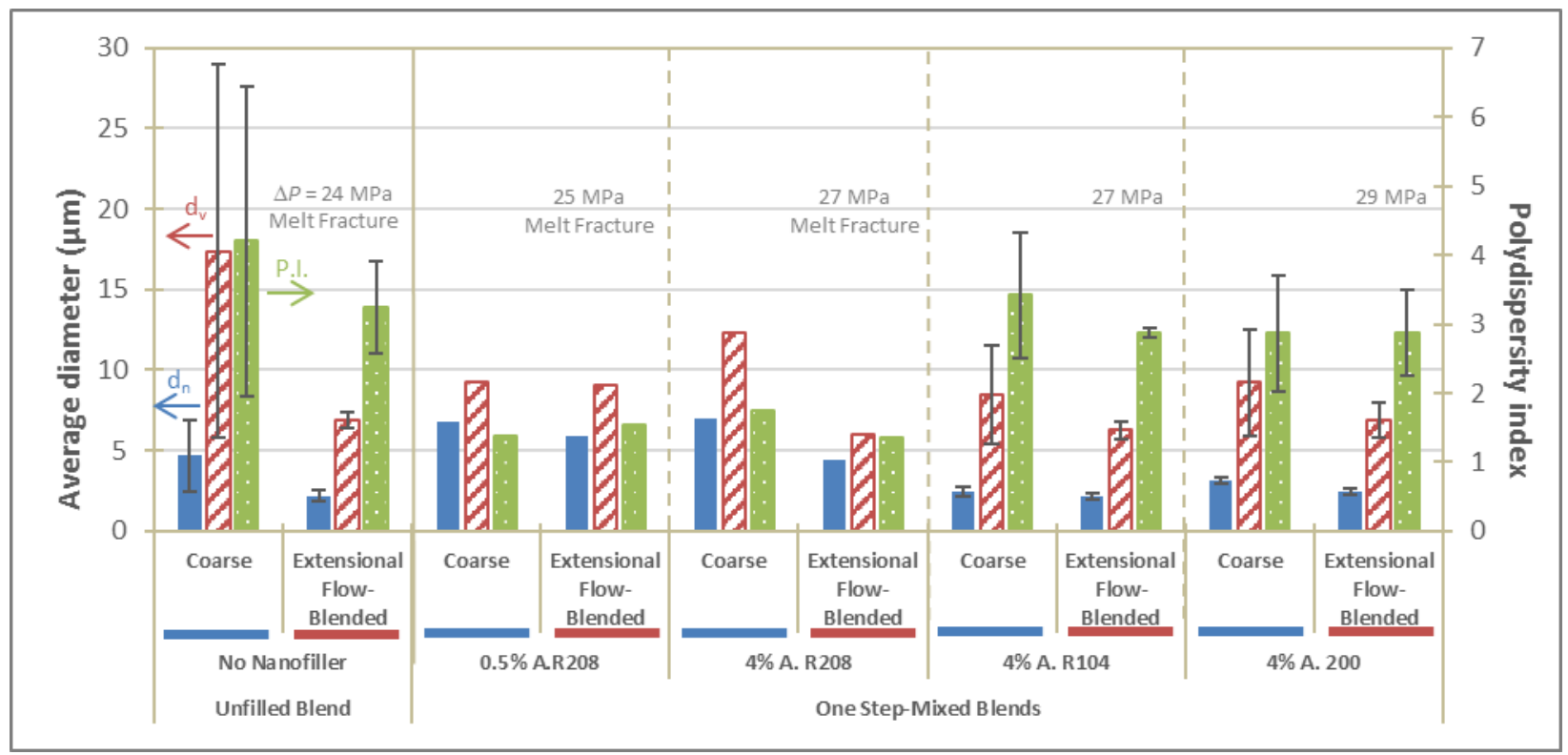

Figure 4-73: Morphology of unfilled \& one step-mixed blends after extensional flow. Extensional flow blending was done through the converging cone die with $0.330 \mathrm{~mm}$ exit diameter, $\varepsilon=7.1$, at $100 \mathrm{~mm} / \mathrm{min}$ piston speed, or $17 \mathrm{~cm}^{3} / \mathrm{min}$ flow rate, at $220^{\circ} \mathrm{C}$. Error bars represent \pm 1 standard deviation across 3 replicate cross sections when applicable.

The effect of extensional flow blending through the converging cone die with $0.330 \mathrm{~mm}$ exit diameter, $\varepsilon=7.1$, at $100 \mathrm{~mm} / \mathrm{min}$ piston speed, or $17 \mathrm{~cm}^{3} / \mathrm{min}$ flow rate, at $220^{\circ} \mathrm{C}$ on the morphology of the two step-mixed nano-filled blend is shown in Figure 4-74. Comparisons to the morphologies of the unfilled and one step-mixed 0.5\% Aerosil R208 in 5\% HDPE/PS blends are also able to be made for the two step-mixed 0.5\% Aerosil 200 in 5\% HDPE/PS blend. Two different nanoparticle grades were used in this comparison to investigate the effects when both blends had the largest driving force available for migration of the nanoparticles to the interface or to the opposite phase of their first loading. To this end, one of the most hydrophobic nanosilica grades, Aerosil R208 with its PDMS coating, was used in one-step mixing, and the hydrophilic nanosilica grade, Aerosil 200, was used in two-step mixing. The final concentration was held constant at $0.5 \%$, but this required a first-step masterbatch of 9.1\% Aerosil 200/HDPE. As shown in Figure 4-69, the coarse blend morphology was much coarser for the two stepmixed blend than that of the one step-mixed blend, due to the very high viscosity and complex 
moduli of flow for the dispersed masterbatch phase. Extensional flow blending of the two stepmixed blend yielded a dramatic reduction in both $d_{v}$ and $d_{n}$.

The morphology of the two step-mixed blend went from being about 4.5-5.0 times coarser than the unfilled blend to being within $40 \%$ with respect to $d_{n}$ and within a factor of 2 with respect to $d_{v}$ after extensional flow blending. Furthermore, interestingly, the pressure drop for extensional flow of the two step-mixed nano-filled blend was actually about $5 \%$ less than that for the unfilled blend. The hypothesis for the reason for this morphological result is that the breakup mechanism of extensional flow blending is thread breakup rather than quasisteady state binary breakup. Extensional flow is adept at stretching dispersed-phase drops into slender threads, and though the coarse drops for the different coarse blends began at much different sizes, the diameter of the elongated threads were likely similar after processing through the axisymmetric extensional flow. Therefore, the final morphologies after thread disintegration due to capillary instabilities are similar. This may also be explained using the capillary number metric. For very coarse two step-mixed nano-filled blends, the coarse blend has very high $\mathrm{Ca}\left(\mathrm{Ca}>>\mathrm{Ca}_{\text {crit }}\right)$ at the initial stage of extensional flow blending. In fact, it is surely higher than the Ca for the unfilled coarse blend at this stage. Therefore, the two step-mixed nano-filled blend has a larger driving force for breakup (for $\mathrm{Ca}$ to approach $\mathrm{Ca}_{\text {crit }}$ ) than does the unfilled blend, and the final morphology of each blend is at similar Ca (still above $\mathrm{Ca}_{\text {crit, }}$ however, due to the transient nature of axisymmetric extensional flow fields among other factors). 


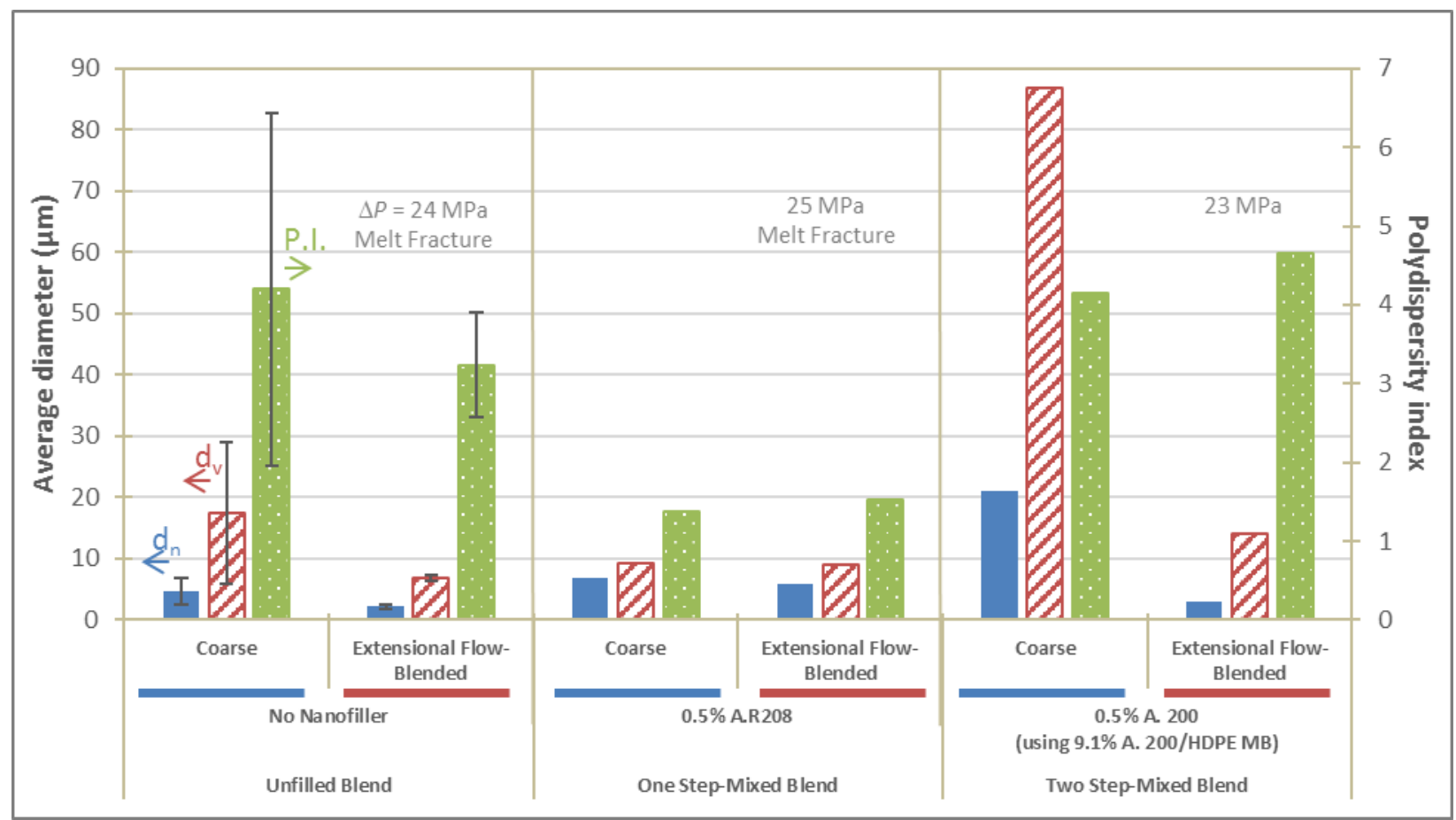

Figure 4-74: Morphology of unfilled \& nano-filled blends after extensional flow. The morphology of unfilled, one step-mixed, and two step-mixed blends before and after extensional flow blending through the converging cone die with $0.330 \mathrm{~mm}$ exit diameter, $\varepsilon=$ $0.330 \mathrm{~mm}$, at $100 \mathrm{~mm} / \mathrm{min}, 17 \mathrm{~cm}^{3} / \mathrm{min}$, and $220^{\circ} \mathrm{C}$. Error bars represent \pm 1 standard deviation across 3 replicate cross sections when applicable.

The one step-mixed and two step-mixed blends of Figure 4-74 were also processed through the other extensional flow cells, and the results of which on morphology are presented in Figure 4-75 using $d_{n}, d_{v}$, and P.I. metrics and in Figure 4-76 using the log-normal $\mu$ and $\sigma$. The morphology of the one step-mixed nano-filled blend after extensional flow was consistent regardless of the extensional flow cell used. Similarly, the morphology of the two step-mixed nano-filled blend after extensional flow was very consistent, with the smallest $d_{\mathrm{v}}$ observed using the converging cone die with $0.330 \mathrm{~mm}$ exit diameter. Extensional flow had a dramatic effect on the $d_{\mathrm{v}}$ and $d_{\mathrm{n}}$ of the two step-mixed blend, and in fact the $d_{\mathrm{n}}$ of that blend was smaller than that of the one step-mixed blend after extensional flow. That is also corroborated by the comparison of the log-normal $\mu$ values of each blend in Figure 4-76. The use of the log-normal metrics also shows that extensional flow blending of the two step-mixed blend decreased the size distribution as well, as elucidated by the decrease in log-normal $\sigma$. 


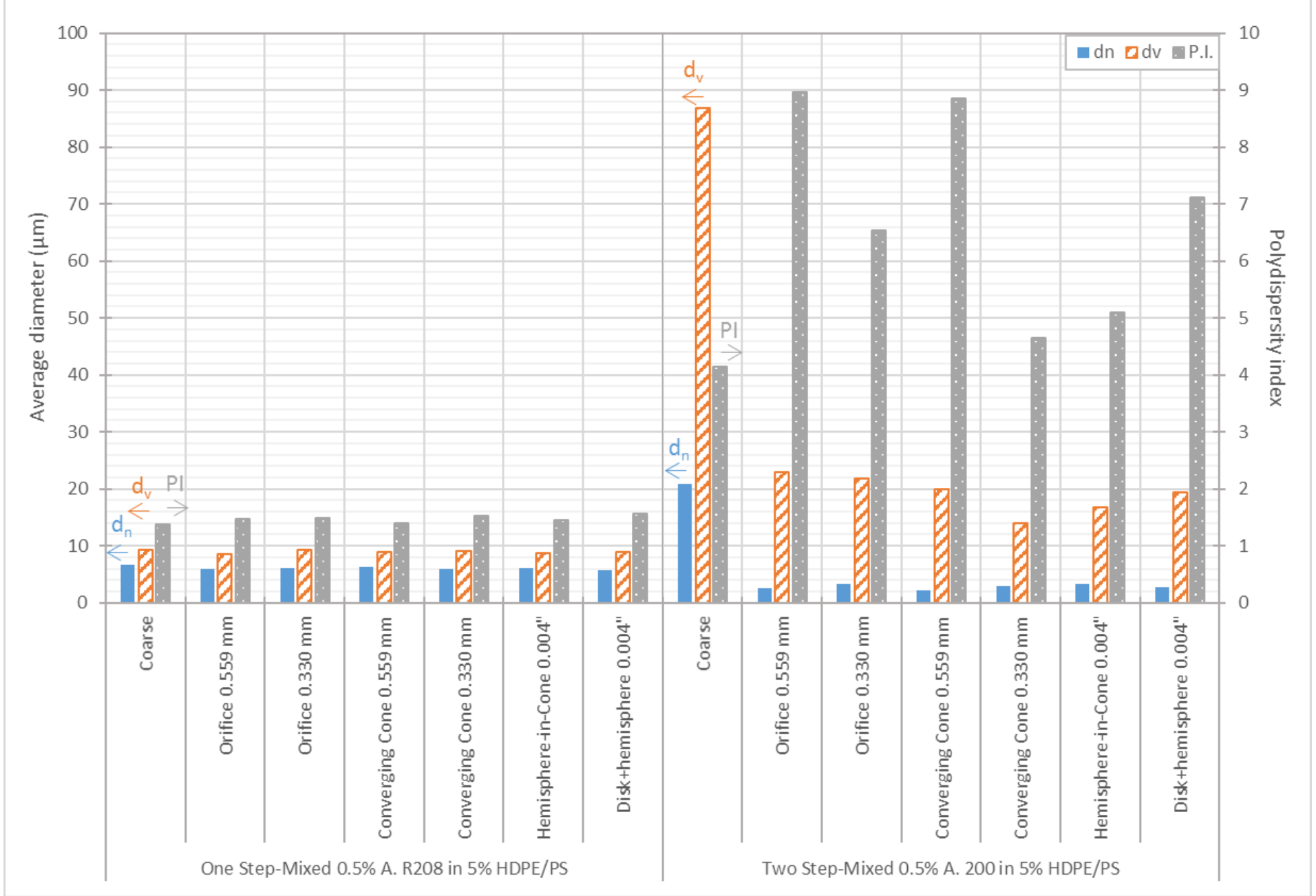

Figure 4-75: Morphology of nano-filled blends through all dies. Morphology as quantified by $d_{n}, d_{v}$, and P.I. of nano-filled blends through all of the extensional flow cells. 


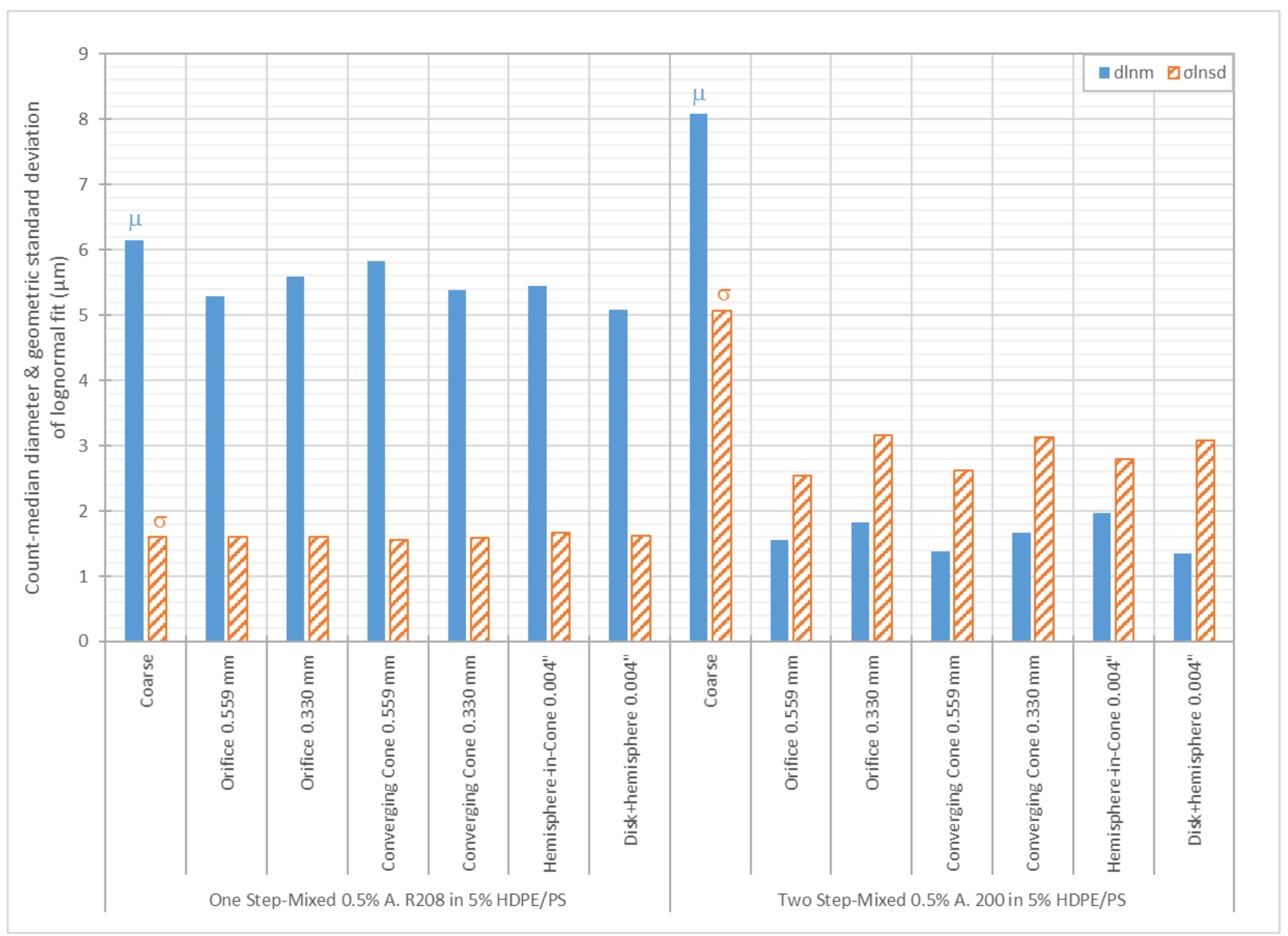

Figure 4-76: Log-normal morphology of nano-filled blends through all dies. Morphology as quantified by log-normal $\mu$ and $\sigma$ of nano-filled blends through all of the extensional flow cells. 
A significant observation was made while imaging the one step-mixed hydrophobic nanosilica-filled blends after extensional flow blending through the converging cone die. Several HDPE dispersed-phase drops had rough surfaces, indicative of solid particulates adsorbed to the surface of the drop. The roughness involved texture on the same scale as the nanoparticle aggregates and is indicative that the nanosilica was migrating to the polymerpolymer interface with extensional flow blending. To fully validate that the roughness was attributed to nanosilica adsorbed to the interface, TEM may be used in future work. Representative images showing this phenomenon are given in Figure 4-77 as well as EDS maps that show that the bulk of the nanosilica remains localized in the PS matrix. The relative amount of solid aggregates observed on the HDPE drops' surface was proportional to the loading of the hydrophobic nanosilica. Specifically, the PDMS-coated nanosilica grade, Aerosil R208, was the grade in the blends in which the migration effect with extensional flow blending was observed. This hydrophobic coating imparts a driving force for the nanoparticles to migrate either to the polymer-polymer interface or to the more nonpolar HDPE phase. 


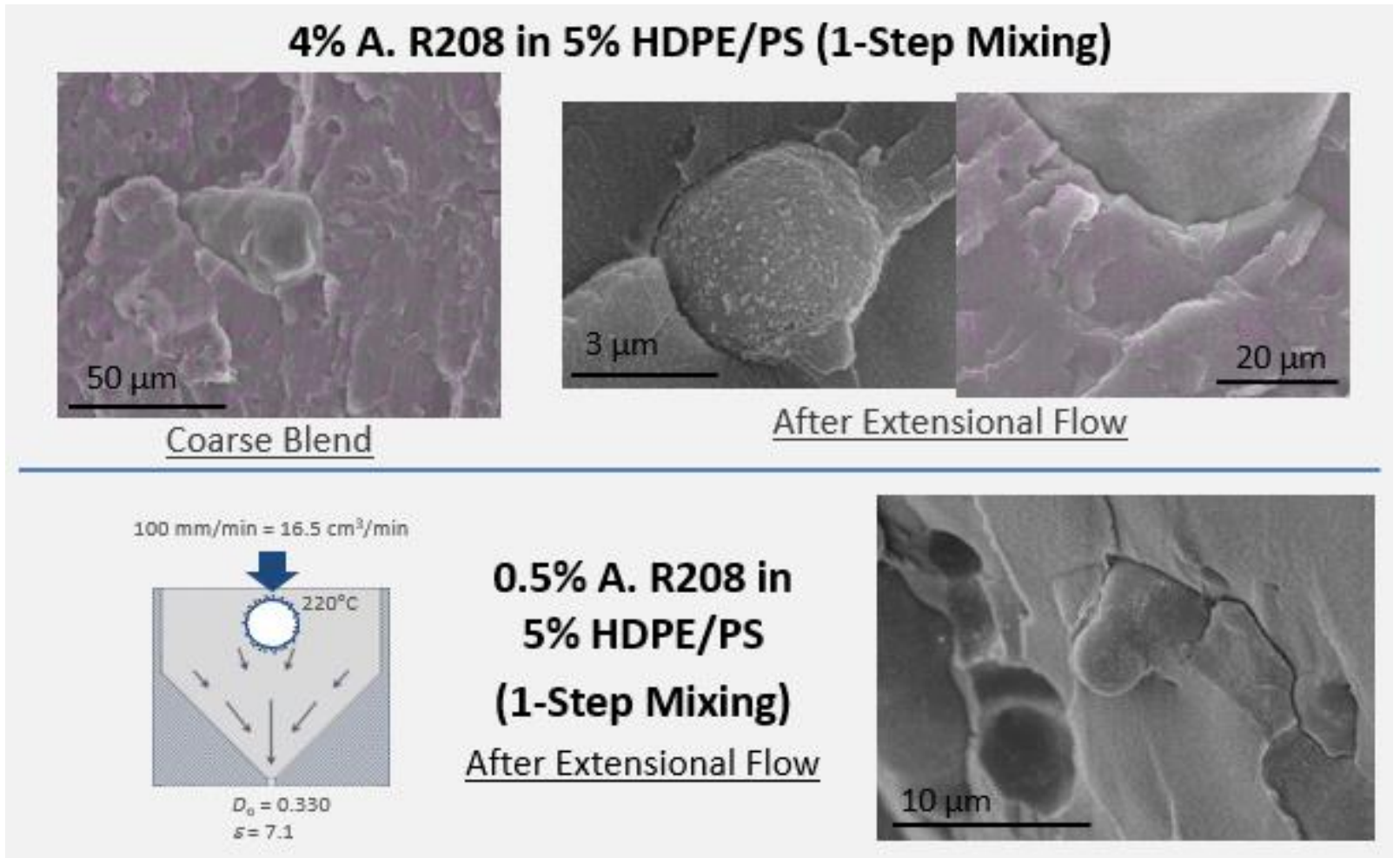

Figure 4-77: Migration of nanoparticles to interface after extensional flow blending. SEM images and EDS maps showing a degree of migration of the nanosilica to the HDPE/PS interface after extensional flow blending through the converging cone die with $0.330 \mathrm{~mm}$ exit diameter, $\varepsilon$ $=0.330 \mathrm{~mm}$, at $100 \mathrm{~mm} / \mathrm{min}, 17 \mathrm{~cm}^{3} / \mathrm{min}$, and $220^{\circ} \mathrm{C}$.

Several factors likely contribute to the appearance of concentration-dependent nanoparticle aggregates at the HDPE/PS interface after extensional flow blending. Firstly, extensional flow blending is known to promote dispersive mixing, both for dispersed polymer phases and for dispersed solid phases. This would impart a higher total surface area of the nanoparticle phase and increase the driving force associated with thermodynamic effects on the nano-filled blends. However, the level of dispersion of the nanoparticle aggregates was qualitatively comparable in both the coarse blends and the extensional flow-blended samples, as observed using TEM. Secondly, extensional flow blending causes breakup and an increase in total surface area of the dispersed polymer phase. This would significantly increase the driving force for thermodynamic effects compared to kinetic effects. Finally, extensional flow blending is adept at stretching out dispersed polymer phase drops into elongated threads. This 
stretching process increases the surface area of the dispersed phase and causes a significant increase in the number of collisions between the dispersed polymer phase and the nanosilica. Once the nanoparticles are able to interact locally with the dispersed HDPE phase, thermodynamics are able to take effect and the nano-phase assumes its thermodynamically predicted phase localization at the polymer-polymer interface.

The overall goal of nanoparticle stabilization was always to modify the polymer-polymer interface with nanoparticles with specially tuned surface properties. At the interface, the nanoparticles may serve as a compatibilizer and stabilize the morphology produced by the physical blending approaches taken in this work. Future work is required to identify whether the fraction of hydrophobic nanosilica aggregates at the HDPE/PS interface after extensional flow blending has any effects on the robustness of the morphology to secondary meltprocessing steps, like injection molding or profile extrusion. The hypothesis of this work is that the nanoparticle aggregates at the HDPE drops' surface will serve as a physical barrier to prevent coalescence from occurring between two colliding drops. Therefore, the nanosilica being localized at the interface is predicted to impart the nanoparticle stabilization effect that was sought after in this work.

\subsubsection{Conclusions for Nanoparticle Stabilization}

The nanoparticles used in this work were chosen based on the following selection criteria:

1) Commercially available and relatively economically favorable,

2) Non-porous surface with well-defined nanoparticle geometry, and

3) Industrial grades with various surface chemistries and properties available. Fumed nanosilica satisfied these selection criteria, with one exception that was not realized until significant studies had already been done: though the primary particles were spherical with non-porous surfaces, the material is received in an agglomerated form comprised of tightly bound aggregates which possess an inherent nano-scale porosity. This is hypothesized to have complicated the study into the thermodynamics effects of the nano-filled blends 
because the first polymer phase to wet these inter-particle pores remains there throughout all subsequent processing.

Effectively dispersing the nanosilica into the polymer blends was challenging, and selectively localizing the nanosilica particles at the polymer-polymer interface using the shear flow-dominated internal mixer proved to be impossible using the mixing strategies of this work. This is likely due to the wetting of the nanosilica aggregates' inter-particle pores by the first molten polymer which contacts them. Therefore, two strategies for the preparation of the coarse blends were realized: 1) one step-mixing, wherein the two polymer phases are premelted then the nanosilica phase is added, and 2) two step-mixing, wherein the nanosilica is premixed into the thermodynamically unpreferred phase to produce a masterbatch, and in a secondary melt mixing step, the masterbatch is mixed into the other polymer. The results of these two mixing strategies were that the nanosilica localized in the PS matrix phase using one step-mixing and it remained in the HDPE dispersed phase using two step-mixing. Furthermore, one-step mixing yielded coarse-blend morphologies that were about a factor of 2 finer than the coarse-blend morphology of the unfilled HDPE/PS blend, and two-step mixing yielded blends with much coarser morphologies, by about a factor of 5 , using the internal mixer.

Extensional flow of nano-filled blends typically produced blend morphologies comparable to that of the unfilled, extensional flow-blended morphology. Also, the pressure drop for this processing was comparable for nano-filled and unfilled blends. This indicates that no coupled or synergistic effects are likely between extensional flow blending and nanoparticle stabilization with respect to morphology development of the dispersed polymer phase. However, one interesting result was observed where the nanosilica phase localization within the blends was dependent on extensional flow processing. The polymer-polymer interface was typically completely devoid of nanoparticles in the one step-mixed coarse blends after processing by a shear flow-dominated process, even though the interface was the thermodynamically predicted localization of the surface-treated nanosilica grades. After extensional flow blending, though, the polymer-polymer interface was observed to have nanoparticles bound to it. The majority of the nanoparticles still localized in the PS matrix phase in these one step-mixed, extensional flow-blended systems, but the relative amount that 
bound to the interface after extensional flow appeared to depend on the loading of nanosilica in the blend, as would be expected. The expected significance of this result is that the nanoparticles may serve as a compatabilizer in this case, since they do modify the polymerpolymer interface, and they may serve their purpose of stabilizing the fine morphologies produced by extensional flow blending against coarsening in post-processing in secondary melt processes. 


\section{Conclusions and Recommendations}

\subsection{Conclusions}

In this dissertation, the effects of extensional flow blending on the morphology of immiscible polymers with high viscosity ratios were investigated. Additionally, the effects of the incorporation of nanoparticles into the blends were also studied, as well as the combined effects of processing nano-filled blends with extensional flow.

5\% HDPE/PS was chosen as the polymer blend to study because it yielded a viscosity ratio greater than 3.8 for all shear rates anticipated in processing, had a significant interfacial tension, and had a sufficiently low concentration of the dispersed phase to more adeptly focus on the effects of processing on drop deformation and breakup rather than coalescence. The blends were processed through in-house fabricated EFCs of various geometries. Initial findings using converging cone dies showed that the total extensional strain in the EFC yielded incrementally finer dispersed-phase morphologies but at the expense of pressure drop. Furthermore, increasing flow rate quickly decreased phase size with an optimum observed at an intermediate flow rate where the residence time in the convergence was not too short compared to the elasticity of the dispersed phase.

The entry profile into the converging nozzle was not found to have a significant effect with this blend, when comparing orifice dies with a $180^{\circ}$ cone angle to converging cone dies with a $45^{\circ}$ cone half-angle, but the addition of inserts that nested within the nozzle were advantageous. The disk-in-tube insert produced excessive shear and did not affect the dispersed-phase size enough to compensate for the very large pressure drop required. The hemisphere-in-cone insert, however, showed versatility because the gap the added stretching episode between the hemisphere and the cone wall was changeable based on spacers added to the shoulder upon which the insert rested. The EFC produced morphologies comparable to those using a converging cone die with greater total extensional strain but requiring a lower pressure drop. Finally, the disk + hemisphere die proved to be difficult to use properly, and like the disk-in-tube die, it did not affect the dispersed-phase morphology enough to compensate for the large pressure drop it required. 
When nanoparticles with various surface chemistries were added to the blends, controlling their localization in the blends proved to be difficult, and kinetic and physical mixing effects were found to dominate over thermodynamic effects. In the preparation of coarse blends in the internal mixer, nanosilica modification and selective localization of the HDPE/PS interface was never achieved, even though all of the surface-treated grades of nanosilica were predicted thermodynamically to localize there. The order of mixing was found to be very important though for the dispersed-phase morphology of these nano-filled coarse blends. Using one-step mixing, in which the two polymers were premelted then nanosilica added, the nanosilica localized exclusively in the PS matrix phase, likely because it was the dominant phase by volume and because it had much lower viscosity which allowed it to flow and wet the nanoparticles more readily than the high-viscosity HDPE dispersed phase. Nanosilica in the matrix phase was found to significantly decrease dispersed-phase size, by about a factor of 2 . Inversely, using two-step mixing, in which the nanosilica was preloaded into the HDPE thermodynamically unpreferred phase then blended with the PS matrix phase in a second melt processing step, the nanosilica remained exclusively localized in the HDPE phase even though it was thermodynamically unpreferred. The dispersed-phase size for those two step-mixed coarse blends was very coarse as well, about 5 times coarser than the unfilled coarse blend, very likely because the dispersed-phase viscosity and elasticity increased greatly with the relatively high loading of nanosilica in the HDPE. Furthermore, because the nanosilica remained localized in whichever phase wetted the surface of its aggregates first, it is hypothesized that the first-wetting polymer phase becomes entrapped in the nanoparticle aggregate and effectively precludes the other phase from the partial wetting of the surface that would be required to selectively bind to the polymer-polymer interface.

Extensional flow blending of nanoparticle-filled blends produced blends with dispersed polymer-phase morphology comparable to those of the extensional flow-blended, unfilled blends. Therefore, no coupled effects were observed of extensional flow and nanoparticle stabilization on the morphology development after the processing of this work using the materials investigated. Extensional flow blending did appear to have an effect on the nanoparticle localization in the blends though. The amount of nanoparticles observed via SEM 
on the surface of dispersed HDPE drops in the one step-mixed blends of hydrophobically surface-treated nanosilica significantly increased after extensional flow blending through the converging cone die, and the relative amount of nanoparticles at the polymer-polymer interface appeared to correlate to the loading of nanoparticles in the blend. This result is hypothesized to prove valuable to capacitate the nanoparticle phase to effectively stabilize the fine dispersed polymer-phase morphology that was produced using extensional flow blending. This nanoparticle stabilization effect then would stabilize the morphology from coalescence in secondary melt-processing steps, like injection molding or profile extrusion.

\subsection{Recommendations}

While the conclusions of this work are expected to significantly advance the academic knowledge of the two polymer compounding mechanisms studied as well as their interactions, namely extensional flow blending, nanoparticle stabilization, and extensional flow blending of nanoparticle-filled blends, application of these conclusions on material systems other than those studied here and industrial acceptance of these blending routes will require a significant amount of future research and development. The following list captures the most pertinent tasks that the author suggests to follow:

- Measure the effects of various morphologies on the properties of the blend to complete the processing-structure-properties relationships that would be of real use to polymer scientists and engineers in industry. This should be done using the blend system studied here as well as any subsequent blend systems that are studied, and the properties of interest will depend on the typical uses for such blend systems, but would likely be dominated by mechanical properties. These may be studied at a lab-scale using a dynamic mechanical analyzer (DMA), which is able to perform mechanical tests such as cantilever-beam bending, double-cantilever beam bending, and torsion using very small samples to measure various modulus values at different temperatures and to measure the mechanical-response glass-transition temperature $\left(T_{\mathrm{g}}\right)$. Thermo-mechanical analysis (TMA) would also be useful to measure the coefficient of thermal expansion (CTE) and 
mechanical-response $T_{\mathrm{g}}$. Furthermore, an in-depth characterization of rheological properties measured using a parallel-plate rheometer and a capillary rheometer is appropriate. Additional thermal properties may also be measured using thermogravimetric analysis (TGA) and differential scanning calorimetry (DSC). DSC is useful to measure the thermal-response $T_{\mathrm{g}}$ of each phase as well as crystallinity and crystallization kinetics of the semicrystalline HDPE phase.

- Study other polymer blend systems with a range of material, chemical, and rheological properties to develop general processing-structure and even processing-structureproperties relationships. The relationships identified in this work should be validated on other blend systems to generalize the findings for all immiscible polymer blends or welldefined sets of immiscible polymer systems. The characteristic properties of the subsequent polymer blend systems should be varied systematically to ensure that useful and attainable relationships may be characterized.

- Optimize the design of EFCS using computational methods validated by experimentation. The hemisphere-in-cone die is the optimum geometry identified in this work, but it is not expected to be the global optimum for EFC designs. Computational modelling and simulation are proficient tools for optimization, and the framework for the fabrication and testing of novel designs has been established in this work. The scope and significance of this work should be maintained within reason to ensure that designs are simple enough for ease of use so that polymer scientists and engineers may understand them more fully. This will enhance the adoption of this technology in industry.

- Employ more complicated methods to attempt to thermodynamically bind the nanoparticles to the polymer-polymer interface. The efforts of this work were not successful in binding the nanoparticles to the interface between the polymers because kinetic effects greatly outweighed thermodynamic effects. Other strategies were conceived of, however, but not attempted due to their complexity. These include the following: use a three-step mixing method in which the nanoparticles are first wetted by PS, then mixed into HDPE to create a masterbatch, then blended into a PS matrix; better disperse the nanoparticle aggregates using ultrasonication in suspension before 
mixing to break down the aggregates toward primary particles and eliminate the interparticle porosity and increase the overall nanoparticle surface area; treat the nanoparticles with other chemistries including reactive chemistries both prior to mixing and in situ in mixing; more closely attempt to replicate successful literature methods; and use other nanoparticles, including carbon black, alumina, clay nanoplatelets, carbon nanotubes, and graphene.

- Expand the theoretical model for the localization of nanoparticles in a binary polymer blend. Several assumptions to the calculation of a wettability parameter as a thermodynamic model to predict the localization of a nanoparticle phase in a two-phase polymer blend are not typically realized using real materials and real processes. The effects of particle-particle interactions are expected to be significant. Tightly bound nanoparticle aggregates contain inter-particle pores in which wetting liquids can become bound by capillary action. In this case, the first wetting polymer is bound to the aggregate's surface and is essentially incorporated into the solid phase. The thermodynamic model should include this phenomenon among other physical phenomena. 


\section{References}

[1] Y. Suzaka, "Mixing device". US Patent 4334783, 1982.

[2] X. Nguyen and L. Utracki, "Extensional flow mixer". US Patent 5451106, 1995.

[3] S. Eggen and R. Nygaard, "Mixing device". US Patent 6299342, 2001.

[4] L. Utracki, Polymer Blends Handbook, Vols. 1-2, Dordrecht, The Netherlands: Kluwer Academic Publishers, 2002.

[5] L. Robeson, Polymer Blends: A Comprehensive Review, Munich: Carl Hanser Verlag, 2007.

[6] A. G. R. Kumar, Fundamentals of polymer engineering: Second edition revised and expanded, New York: Marcel Dekker, Inc., 2003.

[7] H. Meijer, P. Lemstra and P. Elemans, "Structured polymer blends," Macromol. Chem., Macromol. Symp., vol. 16, pp. 113-135, 1988.

[8] G. Taylor, "The formation of emulsions in definable fields of flow," Proc. R. Soc. Lond. A, vol. 146, pp. 501-523, 1934.

[9] H. Grace, "Dispersion phenomena in high viscosity immiscible fluid systems and application of static mixers as dispersion devices in such systems," Chem. Eng. Comm., vol. 14, pp. 225-277, 1982.

[10] J. Janssen and H. Meijer, "Droplet breakup mechanisms: Stepwise equilibrium versus transient dispersion," J. Rheol., vol. 37, no. 4, pp. 597-608, 1993.

[11] J. Janssen, Dynamics of liquid-liquid mixing, PhD Dissertation. Eindhoven, The Netherlands: Eindhoven University of Technology, 1993.

[12] Rayleigh, "On the capillary phenomena of jets," Proc. R. Soc. Lond., vol. 29, pp. 71-97, 1879.

[13] C. Weber, "Disintegration of liquid jets," Z. Angew. Math. Mech., vol. 11, no. 2, pp. 136159, 1931.

[14] S. Tomotika, "On the instability of a cylindrical thread of a viscous liquid surrounded by another viscous fluid," Proc. R. Soc. Lond., vol. 150, no. 870, pp. 322-337, 1935. 
[15] W. Kuhn, "Spontane Aufteilung von Flüssigkeitszylindern in kleine Kugeln," Kolloid Z., vol. 132, pp. 84-99, 1953.

[16] P. Ghodgaonkar and U. Sundararaj, "Prediction of dispersed phase drop diameter in polymer blends: The effect of elasticity," Polym. Eng. and Sci., vol. 36, no. 12, pp. 16561665, 1996.

[17] R. Gupta and T. Sridhar, "Elongational rheometers," in Rheological Measurement, 2nd Edition ed., A. Collyer and D. Clegg, Eds., Dordrecht, Springer Science+Business Media, 1998, pp. 516-549.

[18] D. Khakhar and J. Ottino, "Breakup of liquid threads in linear flows," Internat. J. Multiphase Flow, vol. 13, no. 1, pp. 71-86, 1987.

[19] H. Stone and L. Leal, "Relaxation and breakup of an initially extended drop in an otherwise quiescent fluid," J. Fluid Mechanics, vol. 198, pp. 399-427, 1989.

[20] A. Chesters, "The modelling of coalescence processes in fluid-fluid dispersions: A review of current understanding," Trans. IChemE., vol. 69, no. A, pp. 259-270, 1991.

[21] Y. Chevalier and M.-A. Bolzinger, "Emulsions stabilized with solid nanoparticles: Pickering emulsions," Colloids and Surfaces A: Physiochem. Eng. Aspects, vol. 439, pp. 23-34, 2013.

[22] T. Cheng, H. Keskkula and D. Paul, "Property and morphology relationships for ternary blends of polycarbonate, brittle polymers and an impact modifier," Polymer, vol. 33, no. 8, pp. 1606-1619, 1992.

[23] F. Fenouillot, P. Cassagnau and J.-C. Majesté, "Uneven distribution of nanoparticles in immiscible fluids: Morphology development in polymer blends," Polymer, vol. 50, pp. 1333-1350, 2009.

[24] L. Elias, F. Fenouillot, J. Majeste and P. Cassagnau, "Morphology and rheology of immiscible polymer blends filled with silica nanoparticles," Polymer, vol. 48, pp. 60296040, 2007. 
[25] L. Elias, F. Fenouillot, J.-C. Majesté, G. Martin and P. Cassagnau, "Migration of nanosilica particles in polymer blends," J. Polym. Sci.: Part B: Polym. Phys., vol. 46, pp. 1976-1983, 2008.

[26] L. Elias, F. Fenouillot, J.-C. Majesté, P. Alcouffe and P. Cassagnau, "Immiscible polymer blends stabilized with nano-silica particles: Rheology and effective interfacial tension," Polymer, vol. 49, pp. 4378-4385, 2008.

[27] H. Kammler, G. Beaucage, R. Mueller and S. Pratsinis, "Structure of flame-made silica nanoparticles by ultra-small-angle x-ray scattering," Langmuir, vol. 20, pp. 1915-1921, 2004.

[28] L. Forny, I. Pezron, K. Saleh, P. Guigon and L. Komunjer, "Storing water in powder form by self-assembling hydrophobic silica nanoparticles," Powder Technology, vol. 171, pp. 15-24, 2007.

[29] C. Zhu, Q. Yu, R. Dave and R. Pfeffer, "Gas fluidization characteristics of nanoparticle agglomerates," American Institute of Chemical Engineers AIChE Journal, vol. 51, pp. 426439, 2005.

[30] A. Shenoy, "From melt flow index to rheogram," Rheologica acta, vol. 22, no. 1, pp. 90101, 1983.

[31] F. Morrison, Understanding Rheology, Oxford University Press, 2001.

[32] A. Finniss, Polylactic acid-based polymer blends for durable applications. PhD Dissertation, Morgantown, WV: West Virginia University, 2014.

[33] R. Gupta, Polymer and Composite Rheology, 2nd Ed., New York: Marcel Dekker, Inc., 2000, p. Ch. 17.

[34] R. Gupta, J. Casasnovas, S. Agarwal, M. Padmanabhan and D. Song, "Extensional flow mixing system". USA Patent US 2015/0157993 A1, Filed Dec. 4, 2014. Published Jun. 11, 2015. 
[35] O. Franzheim, M. Stephan, T. Rische, P. Heidemeyer, U. Burkhardt and A. Kiani, "Analysis of morphology development of immiscible blends in a twin screw extruder," Advances in Polymer Technology, vol. 16, no. 1, pp. 1-10, 1998.

[36] L.-Y. Yang, D. Bigio and T. Smith, "Melt blending of linear low-density polyethylene and polystyrene in a Haake internal mixer. II. Morphology-processing relationships," J. Appl. Polym. Sci., vol. 58, pp. 129-141, 1995.

[37] J. Russ, Practical Stereology, New York: Plenum Press, 1986, p. Ch. 4.

[38] E. Underwood, Quantitative Stereology, Reading, MA: Addison-Wesley, 1970, p. Ch. 5.

[39] F. Alsewailem, The influence of processing conditions on the dispersed phase size in immiscible polymer blends. MS Thesis, Morgantown, WV: West Virginia University, 1998.

[40] U. Sundararaj and C. Macosko, "Drop breakup and coalescence in polymer blends: The effects of concentration and compatibilization," Macromolecules, vol. 28, pp. 2647-2657, 1995.

[41] H. Potente, M. Bastian, A. Gehring, M. Stephan and P. Potschke, "Experimental investigation of the morphology development of polyblends in corotating twin-screw extruders," J. Appl. Polym. Sci., vol. 76, pp. 708-721, 2000.

[42] J. Keitz, J. Barlow and D. Paul, "Polycarbonate blends with styrene/acrylonitrile copolymers," J. Appl. Polym. Sci., vol. 29, pp. 3131-3145, 1984.

[43] R. Greco and A. Sorrentino, "Polycarbonate/ABS blends: A literature review," Advances in Polym. Tech., vol. 13, no. 4, pp. 249-258, 1994.

[44] A. Göldel, G. Kasaliwal and P. Pötschke, "Selective localization and migration of multiwalled carbon nanotubes in blends of polycarbonate and poly(styrene-acrylonitrile)," Macromol. Rapid Commun., vol. 30, pp. 423-429, 2009.

[45] G. Merfeld and D. Paul, "Thermodynamic characterization of polymer-polymer interfaces," Macromol. Symp., vol. 159, pp. 105-112, 2000.

[46] J. Kim and C. Kim, "Changes in the interfacial properties of PC/SAN blends with compatibilizer," J. Appl. Polym. Sci., vol. 89, pp. 2649-2656, 2003. 
[47] V. Watkins and S. Hobbs, "Determination of interfacial tensions between BPA polycarbonate and styrene-acrylonitrile copolymers from capillary thread instability measurements," Polymer, vol. 34, no. 18, pp. 3955-3959, 1993.

[48] T. Callaghan, K. Takakuwa, D. Paul and A. Padwa, "Polycarbonate-SAN copolymer interaction," Polymer, vol. 34, no. 18, pp. 3796-3808, 1993.

[49] C. Scott and C. Macosko, "Model experiments concerning morphology development during the initial stages of polymer blending," Polymer Bulletin, vol. 26, no. 3, pp. 341-348, 1991.

[50] C. Scott and C. Macosko, "Morphology development during the initial stages of polymerpolymer blending," Polymer, vol. 36, no. 3, pp. 461-470, 1995.

[51] L. Utracki and P. Sammut, "Rheological evaluation of polystyrene/polyethylene blends," Polym Eng \& Sci, vol. 28, no. 21, pp. 1405-1415, 1988.

[52] S. Wu, "Interfacial and surface tensions of polymer melts and liquids," in Polymer Interface and Adhesion, New York, Marcel Dekker, Inc., 1982, p. Chapter 3.

[53] Z. An, C. Xie, Y. Jiang, F. Zheng and Y. Zhang, "Significant suppression of space charge injection into linear low density polyethylene by surface oxyfluorination," J. Appl. Physics, vol. 106, pp. 104112-1-4, 2009.

[54] E. Arashiro and N. Demarquette, "Use of the pendant drop method to measure interfacial tension between molten polymers," Materials Research, vol. 2, no. 1, pp. 23-32, 1999.

[55] A. Carré and V. Lacarrière, "Cell adhesion to polystyrene substrates: Relevance of interfacial free energy," Contact Angle, Wettability and Adhesion, vol. 5, pp. 253-267, 2008.

[56] J. Comyn, D. Blackley and L. Harding, "Contact angles of liquids on films from emulsion adhesives, and correlation with the durability of adhesive bonds to polystyrene," Int. J. Adhesion and Adhesives, vol. 13, no. 3, pp. 163-171, 1993. 
[57] D. Kwok, L. Cheung, C. Park and A. Neumann, "Study on the surface tensions of polymer melts using axisymmetric drop shape analysis," Polym. Engr. and Sci., vol. 38, no. 5, pp. 757-764, 1998.

[58] B. Natarajan, Y. Li, H. Deng, L. Brinson and L. Schadler, "Effect of interfacial energies on dispersion and glass transition temperature in polymer nanocomposites," Macromolecules, vol. 46, pp. 2833-2841, 2013.

[59] D. Owens and R. Wendt, "Estimation of the surface free energy of polymers," J. Appl. Polym. Sci., vol. 13, pp. 1741-1747, 1969.

[60] S. Wu, "Surface and interfacial tensions of polymer melts, II. Poly(methyl methacrylate), poly(n-buytl methacrylate), and polystyrene," J. Physical Chemistry, vol. 74, no. 3, pp. 632638, 1970.

[61] M. Tang, S. Agarwal, F. Alsewailem and R. Gupta, "Effect of nanoplatelets and polymer crystallinity on moisture diffusion through polylactic acid," in Proc. Soc. Plast. Eng. ANTEC, Las Vegas, April 28-30, 2014.

[62] C. Vasile and M. Pascu, Practical Guide to Polyethylene, Shawbury, UK: Rapra Technology Limited, 2005.

[63] M. Spencer, L. Cui, Y. Yoo and D. Paul, "Morphology and properties of nanocomposites based on HDPE/HDPE-g-MA blends," Polymer, vol. 51, pp. 1056-1070, 2010.

[64] D. Zhou, P. Yue and J. Feng, "Viscoelastic effects on drop deformation in a converging pipe flow," J. Rheol., vol. 52, no. 2, pp. 469-487, 2008.

[65] Z. Starý, F. Machui and H. Münstedt, "Elongational creep experiments - A new method for investigations of morphology development in polymer blends," Polymer, vol. 51, pp. 37443752, 2010.

[66] K. Min, J. White and J. Fellers, "High density polyethylene/polystyrene blends: Phase distributive morphology, rheological measurements, extrusion, and melt spinning behavior," J. Appl. Polym. Sci., vol. 29, pp. 2117-2142, 1984. 
[67] J. Li and B. Favis, "Strategies to measure and optimize the migration of the interfacial modifier to the interface in immiscible polymer blends," Polymer, vol. 43, pp. 4935-4945, 2002.

[68] C. Chen and J. White, "Compatibilizing agents in polymer blends: Interfacial tension, phase morphology, and mechanical properties," Polym. Eng. and Sci., vol. 33, no. 14, pp. 923930, 1993.

[69] B. Avitzur, "Strain-hardening and strain-rate effects in plastic flow through conical converging dies," J. Engineering for Industry, vol. 89, no. 3, pp. 556-562, 1967.

[70] R. Larson, "Instabilities in viscoelastic flows," Rheol. Acta, vol. 31, pp. 213-263, 1992.

[71] M. Meller, A. Luciani and J.-A. Månson, "Flow through a convergence. Part 2: Mixing of high viscosity ratio polymer blends," Polym. Eng. and Sci., vol. 42, no. 3, pp. 634-653, 2002.

[72] S. Wu, "Formation of dispersed phase in incompatible polymer blends: Interfacial and rheological effects," Polym. Eng. and Sci., vol. 27, no. 5, pp. 335-343, 1987.

[73] B. Yaghi, M. Benayoune and A. Al-Bemani, "Viscosity of water-oil emulsions with added nano-size particles," Petroleum Science and Technology, vol. 19, no. 3, pp. 373-386, 2001.

[74] P. Thareja and S. Velankar, "Interfacial activity of particles at PI/PDMS and PI/PIB interfaces: analysis based on Girifalco-Good theory," Colloid Polym. Sci., vol. 286, pp. 1257-1264, 2008.

[75] J. Vermant, G. Cioccolo, K. Golapan Nair and P. Moldenaers, "Coalescence suppression in model immiscible polymer blends by nano-sized colloidal particles," Rheol. Acta, vol. 43, pp. 529-538, 2004.

[76] C. Whitby, D. Fornasiero and J. Ralston, "Effect of adding anionic surfactant on the stability of Pickering emulsions," Journal of Colloid and Interface Science, vol. 329, pp. 173181, 2009. 
[77] F. Galindo-Rosales, F. Rubio-Hernandez and J. Velazquez-Navarro, "Shear-thickening behavior of Aerosil R816 nanoparticles suspensions in polar organic liquids," Rheol. Acta, vol. 48, pp. 699-708, 2009.

[78] R. Myers and H. Clever, "Surface tension of octamethylcyclotetrasiloxane and hexamethyldisilazane and their solutions with carbon tetrachloride and n-hexadecane," Journal of Chemical and Engineering Data, vol. 14, no. 2, pp. 161-164, 1969.

[79] "Surface-Tension.de," Last updated November 20, 2007. [Online]. Available: http://surface-tension.de/solid-surface-energy.htm. [Accessed 29 April 2016].

[80] J. Bohm, J. Haubelt, P. Henzi, K. Litfin and T. Hanemann, "Tuning the refractive index of polymers for polymer waveguides using nanoscaled ceramics or organic dyes," Advanced Engineering Materials, vol. 6, pp. 52-57, 2004.

[81] J. Palierne, "Linear rheology of viscoelastic emulsions with interfacial tension," Rheol. Acta, vol. 29, pp. 204-214, 1990.

[82] C. Lacroix, M. Aressy and P. Carreau, "Linear viscoelastic behavior of molten polymer blends: A comparative study of the Palierne and Lee and Park models," Rheol. Acta, vol. 36, pp. 416-428, 1997.

[83] C. M. P. Tucker, "Microstructural evolution in polymer blends," Annu. Rev. Fluid Mech., vol. 34, pp. 177-210, 2002.

[84] M. B. M. Iza, "Nonlinear rheology of immiscible polymer blends: step strain experiments," J. Rheol., vol. 44, no. 6, pp. 1363-1384, 2000.

[85] T. Forsyth, "Converging flow of polymers," Polymer-Plastics Technology and Engineering, vol. 6, no. 1, pp. 101-131, 1976.

[86] P. Van Puyvelde and P. Moldenaers, "Rheology and morphology development in immiscible polymer blends," in Rheology Reviews, Aberystwyth, The British Society of Rehology, 2005, pp. 101-145.

[87] J. Elmendorp, "Dispersive mixing in liquid systems," in Mixing in Polymer Processing, New York, Marcel Dekker, Inc., 1991, p. Ch. 2. 
[88] L. Erwin, "Principles of laminar fluid/fluid mixing," in Mixing in Polymer Processing, New York, Marcel Dekker, Inc., 1991, p. Ch. 1.

[89] M. Tokihisa, K. Yakemoto, T. Sakai and e. al., "Extensional flow mixer for polymer nanocomposites," Polym. Eng. and Sci., vol. 46, pp. 1040-1050, 2006.

[90] R. Gupta, E. Kennel and K. Kim, Polymer Nanocomposites Handbook, Boca Raton, FL: Taylor and Francis Group, LLC, 2010.

[91] B. Yaghi, M. Benayoune and A. Al-Bemani, "Viscosity of water-oil emulsions with added nano-sized particles," Petroleum Science and Technology, vol. 19, pp. 373-386, 2001.

[92] D. Graebling, R. Muller and J. Palierne, "Linear viscoelastic behavior of some incompatible polymer blends in the melt: Interpretation of data with a model of emulsion of viscoelasic liquids," Macromolecules, vol. 26, pp. 320-329, 1993.

[93] P. Van Puyvelde, S. Velankar and P. Moldenaers, "Rheology and morphology of compatibilized polymer blends," Current Opinion in Colloid \& Interface Sci., vol. 6, pp. 457463, 2001. 